 \\ ESES
}

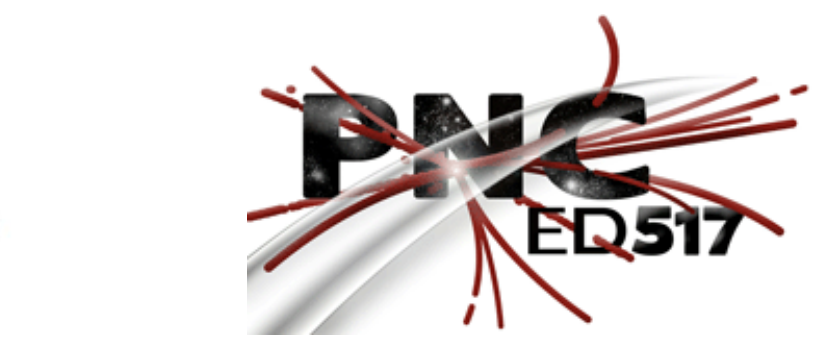

\section{UNIVERSITÉ PARIS-SUD}

ÉCOLE DOCTORALE 517 :

PARTICULES, NOYAUX ET COSMOS

Laboratoire : CSNSM-IN2P3-CNRS, UMR 8609

\section{THĖSE DE DOCTORAT}

\author{
PHYSIQUE
}

par

\author{
Vladimir MANEA
}

\section{Penning-trap mass measurements of exotic rubidium and gold isotopes for a mean-field study of pairing and quadrupole correlations}

Date de soutenance :

$29 / 09 / 2014$

\section{Composition du jury :}

Directeur de thèse :

Rapporteurs :

Examinateurs :
David LUNNEY

Héloïse GOUTTE

Piet VAN DUPPEN

Brigitte ROUSSIÈRE Hervé SAVAJOLS Nigel ORR
Directeur de Recherche CNRS, CSNSM, Orsay, France

Ingénieure Physicienne, CEA/DSM, Centre de Saclay, France Professeur, KU, Leuven, Belgique

Directrice de Recherche CNRS, IPN, Orsay, France Chargé de Recherche CNRS, GANIL, Caen, France Directeur de Recherche CNRS, LPC, Caen, France 



\begin{abstract}
The most complex nuclei are situated between the magic and the mid-shell ones, in regions known for sudden changes of the trends of nuclear observables. These are the so-called shape-transition regions, where the nuclear paradigm changes from the vibrational liquid drop to the static rotor. With few exceptions, nuclei in these regions are radioactive, with half-lives dropping into the millisecond range.

Complementing the information obtained from the low-lying excitation spectrum, nuclear binding energies and mean-square charge radii are among the observables most sensitive to these changes of nuclear structure. In the present work, a study of the shapetransition phenomenon is performed by measurements of radioactive nuclides produced by the ISOLDE facility at CERN. The masses of the neutron-rich rubidium isotopes ${ }^{98-100} \mathrm{Rb}$ and of the neutron-deficient gold isotopes ${ }^{180,185,188,190,191} \mathrm{Au}$ are determined using the Penning-trap mass spectrometer ISOLTRAP. The mass of ${ }^{100} \mathrm{Rb}$ is determined for the first time. Significant deviations from the literature values are found for the isotopes ${ }^{188,190} \mathrm{Au}$. A new experimental method is presented, using a recently developed multi-reflection time-of-flight mass spectrometer as a beam-analysis tool for resonanceionization laser spectroscopy. The new method opens the path to measurements of atomic hyperfine spectra with ISOLTRAP, from which charge radii and electromagnetic moments of radioactive nuclides can be extracted.

The properties of the studied nuclides map the borders of two prominent regions of quadrupole deformation, which constrain the fine balance between pairing and quadrupole correlations in the nuclear ground states. This balance is studied by the Hartree-FockBogoliubov (HFB) approach. The sensitivity of the shape-transition phenomenon to the strength of pairing correlations is demonstrated. In particular, the strong odd-even staggering of charge radii in the mercury isotopic chain is shown to result in the HFB approach from the fine interplay between pairing, quadrupole correlations and quasiparticle blocking.
\end{abstract}





\section{Contents}

$\begin{array}{ll}\text { Acknowledgements } & 1\end{array}$

$\begin{array}{ll}\text { Preamble } & 3\end{array}$

1 Nuclear observables $\quad 9$

1.1 Binding energy . . . . . . . . . . . . . . . . . . . . . . . . . . . . .

1.2 Trends of binding energies . . . . . . . . . . . . . . . . 11

1.2.1 Decomposition and extension of the binding energy function . . . . 12

1.2 .2 Mass filters . . . . . . . . . . . . . . . . . . . 14

1.3 Complementary nuclear data . . . . . . . . . . . . . . . . 25

2 Experimental method and data analysis $\quad 31$

2.1 Charged-particle traps . . . . . . . . . . . . . . . . . . 31

2.2 Penning trap . . . . . . . . . . . . . . . . . . . . 32

2.2.1 Dynamics of a trapped ion . . . . . . . . . . . . . . . 32

2.2.2 Driving the ion's motion . . . . . . . . . . . . . . . . . . . . . . . . . . . . 35

2.2.3 Detecting the ion's motion . . . . . . . . . . . . . . 37

2.3 From cyclotron frequency to mass: procedure, precision, systematic errors . 42

2.4 Production and preparation of the ion ensemble . . . . . . . . . . . . 47

2.5 Complementary applications of the MR-TOF MS . . . . . . . . . . . 55

2.6 Experimental results . . . . . . . . . . . . . . . . . . . . 59

2.6.1 Neutron-rich rubidium isotopes . . . . . . . . . . . . . 59

2.6.2 Neutron-deficient gold isotopes . . . . . . . . . . . . . 64

$\begin{array}{lll}3 & \text { Nuclear-theory concepts } & 71\end{array}$

3.1 Nuclear forces . . . . . . . . . . . . . . . . . . . . . . . 71

3.2 Many-body calculations . . . . . . . . . . . . . . . . . . . . . . . . . . . . 72

3.3 The Hartree-Fock-Bogoliubov approach . . . . . . . . . . . . . . . . 74

3.3.1 Hartree-Fock field . . . . . . . . . . . . . . . . . . . . . 74

3.3 .2 Pairing field . . . . . . . . . . . . . . . . 77 
3.4 Competition between particle-particle and

particle-hole correlations in nuclei . . . . . . . . . . . . . . . . . 80

3.5 Theoretical analysis of the measured nuclear data . . . . . . . . . . . 82

3.5.1 Aim ....................... 82

3.5.2 HFODD code . . . . . . . . . . . . . . . . . . . . . . . . . . . . 84

3.5.3 Tests of the method . . . . . . . . . . . . . 86

4 Self-consistent mean-field calculations $\quad 93$

4.1 Neutron-rich $A \approx 100$ nuclei . . . . . . . . . . . . . . . . . . . 93

4.2 Neutron-deficient gold-thallium nuclei . . . . . . . . . . . . . . . . 103

4.3 Odd-even staggering of mercury isotopes . . . . . . . . . . . . . . . 109

4.4 Summary . . . . . . . . . . . . . . . . . . 116

5 Conclusions and outlook $\quad 121$

$\begin{array}{lr}\text { Appendices } & 125\end{array}$

$\begin{array}{lr}\text { A Finite-difference operators } & 127\end{array}$

B Motion of a charged particle in a Penning trap 131

$\begin{array}{ll}\text { C Publications related to thesis work } & 137\end{array}$ 


\section{List of Figures}

1.1 Experimental binding energies and different mass filters for the calcium isotopes . . . . . . . . . . . . . . . . . 16

1.2 Experimental two-neutron separation energies of neutron-rich $A \approx 100$ nuclei 20

1.3 Experimental two-neutron separation energies and three-point estimator of the odd-even neutron gap for the bismuth and polonium isotopes . . . . . 24

1.4 Experimental two-proton separation energies of the thallium isotopes and three-point estimator of the odd-even neutron gap for the thallium and gold isotopes. . . . . . . . . . . . . . . . . . 25

1.5 Experimental $N=50$ one-neutron and two-neutron gaps as a function of $Z 26$

1.6 Complementary nuclear data for the zirconium isotopic chain . . . . . . . . 29

2.1 Idealized representations of ISOLTRAP's measurement Penning trap . . . 33

2.2 Excitation patterns of the radial eigenmotions of an ion in a Penning trap . 36

2.3 Scans of the frequencies of the main excitations of the ion motion applied at ISOLTRAP in the measurement trap . . . . . . . . . . . . . . . . . 39

2.4 Optimization of the phase and amplitude of some of the excitations of the ions' motion applied at ISOLTRAP in the measurement trap . . . . . . . . 41

2.5 Schematic of the ISOLTRAP setup . . . . . . . . . . . . . 50

2.6 Exemplary scans for the purification techniques used at ISOLTRAP . . . . 53

2.7 Illustrative examples of applications of the MR-TOF MS as a beam analyzer 57

2.8 TOF-ICR resonance of ${ }^{100} \mathrm{Rb}^{+}$obtained with a Ramsey-type excitation pattern . . . . . . . . . . . . . . . . . . 6 62

2.9 TOF-ICR resonance of ${ }^{98} \mathrm{Rb}^{+}$for with a $400 \mathrm{~ms}$ quadrupole excitation time 62

2.10 Two-neutron separation energies of rubidium isotopes using masses from the literature and this work . . . . . . . . . . . . . . . . . . 64

2.11 Two-neutron separation energies and mean-squared charge radii of the gold isotopes using data from the literature . . . . . . . . . . . . . . . . 65

2.12 Resonance-ionization laser spectroscopy of the $6 s^{2} S_{1 / 2} \rightarrow 6 p{ }^{2} P_{1 / 2}$ transition in ${ }^{185} \mathrm{Au} \ldots \ldots \ldots \ldots$. . . . . . . . . . . . . . 68

2.13 Two-neutron separation energies and mean-squared charge radii of the gold isotopes from the literature and this work . . . . . . . . . . . . . . 69 
3.1 Calculated deformation energy of the prolate and oblate configurations, obtained using a spherical and a set of deformed harmonic oscillator bases for the krypton and gold isotopes . . . . . . . . . . . . . . . . . . 89

3.2 Theoretical mean-square charge radii of the prolate configurations along the krypton isotopic chain and oblate configurations along the gold isotopic chain 91

4.1 Deformation energy of the prolate and oblate configurations of the krypton isotopes, using different values of the pairing interaction . . . . . . . . . . . 94

4.2 Experimental two-neutron separation energies and mean-square charged radii of neutron-rich $A \approx 100$ nuclei, compared to HFB-SLy4 calculations . 96

4.3 Experimental two-neutron separation energies and mean-square charge radii of even- $Z$, neutron-rich $A \approx 100$ nuclei, compared to self-consistent meanfield calculations from the literature . . . . . . . . . . . . . . . . . 101

4.4 Theoretical values of the $N=50$ two-neutron shell gap compared to experimental values . . . . . . . . . . . . . . . . . . . 102

4.5 Deformation energy of the prolate and oblate configurations of the gold isotopes using different values of the pairing interaction . . . . . . . . . . 105

4.6 Experimental two-neutron separation energies and mean-square charge radii of neutron-deficient gold-thallium nuclei, compared to HFB-SLy4 calculations 107

4.7 Experimental two-neutron separation energies and mean-square charge radii of neutron-deficient mercury isotopes, compared to self-consistent meanfield calculations from the literature . . . . . . . . . . . . . . . . . . 110

4.8 Experimental values of the five-point empirical pairing gap for gold and mercury isotopes compared to the results of HFB-SLy4 calculations for mercury isotopes . . . . . . . . . . . . . . . . . . 112

4.9 Experimental values of the five-point empirical pairing gap for mercury and gold isotopes compared to the results of HFB-SLy4 calculations for mercury isotopes (only ground-state configuration) . . . . . . . . . . . 113

4.10 Experimental and theoretical two-neutron separation energies and meansquare charge radii of neutron-deficient gold and mercury isotopes, for a pairing interaction of strength $V_{0}=-200 \mathrm{MeV} \mathrm{fm}^{3}$ (with and without quasi-particle blocking) . . . . . . . . . . . . . . . . . . . . 114

4.11 Experimental and theoretical two-neutron separation energies and meansquare charge radii of neutron-deficient gold and mercury isotopes, for a pairing interaction of strength $V_{0}=-225 \mathrm{MeV} \mathrm{fm}^{3}$ (with and without quasi-particle blocking) . . . . . . . . . . . . . . . . . 115 


\section{List of Tables}

2.1 Parameters describing the production, preparation and measurement of the rubidium and gold isotopes discussed in this work . . . . . . . . . . . . 61

2.2 Frequency ratios with respect to ${ }^{85} \mathrm{Rb}$ and mass excess values of the rubidium isotopes measured in this work . . . . . . . . . . . . 63

2.3 Frequency ratios with respect to ${ }^{133} \mathrm{Cs}$ and mass excess values of the gold isotopes measured in this work . . . . . . . . . . . . . . . . . . . 69 



\section{Acknowledgements}

Physics research is nowadays, more than ever before, the collective effort of a growing community of scientists. Beyond any discussion about the ratio of scientific content to academic text and the way it has evolved in some branches of physics, a fact seems certain: very few if any of today's advances, from the most trivial to the most sublime, can any longer be identified as individual achievements. So when we go in front of a jury to defend our work we defend the work of many, although we still practice a formal separation, for the purpose of academic tradition. But at the end, when we draw the line or, rather, before even starting, the contribution of our colleagues should be acknowledged.

I was fortunate to start my work with ISOLTRAP at a time when the local team possessed not only a great range of knowledge and skills to learn from, but also a rare commitment which made the difference, in key moments, for the experiment's success. We had some of ISOLTRAP's finest, to whom I am deeply indebted: Robert Wolf, the Jimmy Page of our group, a craftsman if I ever saw one, the guy you want to be, always up to the challenge and overwhelmingly decisive at times, without ever ceasing to be well grounded and sound; Marco Rosenbusch, the man of a hundred ideas about flying ions, never afraid to try something new and never losing his good spirit, for which we've been so many times rewarded; Frank Wienholtz, the man I learned the most from, the backbone of ISOLTRAP since I've known him, the reason why we never had to worry, even facing our most serious challenge, because he always had the right tool for it. I would like to thank Dinko Atanasov, who started with ISOLTRAP at a time when there was no immediate perspective of scientific results and remained committed all the way and who, together with Christopher Borgmann and Dennis Neidherr, maintained the control system in good shape. I would also like to thank Susanne Kreim for keeping ISOLTRAP well prepared and organized, so that we never had to worry about more than the scientific challenges we were up against. Her work in the lab and the good documentation of it where my first course on how to use the setup. I am grateful to David Lunney, my PhD supervisor, who from the first day regarded me not as his student, but as his younger

colleague, a confidence which I will not forget. Pragmatic without being cynical and fair without being idealistic, he was the supervisor everyone would like to have. Thank you for offering me the opportunity of coming to CERN, with so much flexibility and such generous support. I thank all my colleagues, old and new, who were at CERN during the 
last year, allowing me more time for the writing and preparation of this thesis. We are also all indebted to the many past members of the local team who left us a good setup to work with and who are still available to help for beam times and with valuable advice.

Many thanks to Frank and Dinko, as well as Stefan Kemnitz, who together set up the computing cluster on which the more involved mean-field calculations were performed.

A radioactive-beam experiment starts with the production of the beam itself, often much more complicated than the actual measurements and certainly critical for their success. For their continuous effort of improving the quality of beam production at ISOLDE and for the permanent on-line support, I would like to thank the ISOLDE technical and physics staff, particularly the target, RILIS and operator teams. I am indebted to ISOLDE for welcoming me in the local group and for the generous support received throughout the years spent at CERN. I would also like to acknowledge the French CNRS-IN2P3, the Paris-Sud University, as well as the ENSAR program for their financial support.

Last but not least, I would like to thank the referees and jury of the thesis for their comments and corrections. 


\section{Preamble}

The development of mass spectrographs in the first half of the twentieth century lead to a series of crucial breakthroughs in the understanding of matter at the subatomic level. The discovery of isotopes and of the so-called "whole-number rule" [Ast19; Ast20b] hinted at the existence of the discrete constituents of the atomic nucleus, the proton (recognized as hydrogen nucleus) [Rut19] and neutron [Cha32]. The observation that the mass of helium is less than the sum of four hydrogen masses [Ast20a] offered the key to the energy source of the Sun and all stars, nuclear fusion, and revealed, already in the early twenties, the immense reservoir of "subatomic energy" [Edd20]. Finally the news that the mass of an atom is less than the total mass of its constituents (the socalled "mass defect", initially discovered as a deviation from the whole-number rule and named "packing fraction" [Ast27]), offered the first test of the relativistic energy-mass relationship $\left(E=m c^{2}\right)$ [Coc32].

Each of these discoveries was preceded by an improvement in the resolving power and precision of the mass-measurement techniques that made it possible. Beyond their immediate consequences for theory, they showed the decisive role of technical developments for advancing scientific knowledge and laid out the most important directions of research in mass spectrometry, still valid today. It is with modern installations implemented at radioactive-ion-beam facilities that mass spectrometry continues the tradition of determining nuclear mass defects, started by F. W. Aston almost a century ago, but applied now to exotic nuclides [Lun03]. Pivotal for the formulation of the first shell model [May48; May49] nuclear binding energies still represent one of the most solid empirical bases upon which models of nuclear structure can be formulated and constrained [Gor13] and provide crucial information for the description of astrophysical processes responsible for the synthesis of the elements (the rapid neutron-capture process being perhaps the one of greatest interest [Gor01; Arn07]).

By using advances which allow unprecedented precision, mass spectrometers are also used for a wide range of fundamental-physics studies [Bla10]. To give only a few examples, candidates for the neutrinoless double-beta decay process are sought by the high-precision determination of their corresponding $Q$ values, which influence the exceptionally-long decay half-lives [Fin12]; high-precision $Q$ values of super-allowed beta emitters are among the quantities required to determine the $V_{u d}$ element of the Cabibbo-Kobayashi-Maskawa 
quark-mixing matrix and to test its unitarity, which is one of the main pillars of the Standard Model [Har09]. The relativistic energy-mass relationship itself is being put under closer scrutiny, as shown in a recent report [Rai05], where high-precision mass spectrometry has an important contribution.

The object of this thesis is mass spectrometry of exotic nuclides for the determination of nuclear binding energies. The exotic nuclides are produced by the ISOLDE radioactive ion-beam facility [Kug00] at CERN. ISOLDE is the pioneering Isotope Separation On-Line (ISOL) laboratory, using relativistic protons from CERN's accelerator complex to produce nuclear reactions on thick targets. This method yields exotic nuclides at low energy over the whole range of the nuclear chart, from the lightest elements up to uranium. The masses of these nuclides are determined using the Penning-trap mass spectrometer ISOLTRAP [Muk08], which, as ISOLDE, was the first of its kind in the field of nuclear-structure studies. Installed at ISOLDE in the mid eighties as a tandem of two Penning traps [Sch86], the ISOLTRAP mass spectrometer has since gone through a series of improvements and breakthroughs [Klu13], which have become the standard for similar experiments emerging worldwide. Nowadays a mature experiment moving into its fourth decade of activity, ISOLTRAP continues to perfect itself and push the boundaries of what is achieved and what is achievable in the field of on-line mass spectrometry [Wol13b; Kre13].

The scientific context of this thesis work is that of a generally-recognized need for change of scale in the approach to nuclear-physics research, both experimentally and theoretically. Facilities everywhere have undertaken ambitious projects to build the radioactive ion-beam factories of tomorrow [Blu13]. FAIR at GSI, SPIRAL2 at GANIL, SPES at LNL, HIE-ISOLDE at CERN, ARIEL at TRIUMF and FRIB at MSU are some highlights showing the magnitude of the international effort and the willingness to trade a lot of the possible research activity for one of design and manufacture. The long-term gain might overcompensate the temporary set-back.

In line with these efforts, mass spectrometers are multiplying, to benefit from different ion-production techniques so as to increase the rate at which the masses of new radioactive isotopes can be determined. They are also increasing their sensitivity, efficiency and background-suppression capability, to allow studying beams of less yield and higher contamination [Klu13]. The increasing problem of beam purity has propelled the field of mass spectrometry from what had become a niche of nuclear-physics research (determination of nuclear binding energies) to a main focus for future beam-delivery systems: the exceptional beam-preparation and purification capability, with record-breaking resolving power, recommends mass spectrometers as essential, in one form or another, for many of the existing experimental installations doing nuclear-physics research [Nie02; Vou08; Blu13; Asc14]. Consequently, techniques mastered at ISOLTRAP and other similar experiments around the world are now used in combination with completely different setups, to extend their reach towards the proton and neutron drip lines. This synergy between mass spectrometers and other types of experiments is one of the important ideas 
of nuclear-physics research today (see [Kow12; Mar13] for ISOLTRAP-related examples).

Nuclear theory is also in a continuous search for new approaches. After a few decades of phenomenological modeling of nuclear structure, either through microscopic-macroscopic formulas [Mye66; Str67; Mol95], or large-scale shell model [Cau05] and self-consistent mean-field [Ben03] calculations with effective interactions fitted on nuclear data, the last years have marked the beginning of a change of philosophy. While the main features of the nuclear many-body-problem have already become textbook concepts [Rin00], an accurate description of these phenomena with predictive power is still lacking and arguably constitutes, more than physics beyond the Standard Model, our generation's greatest theoretical challenge. Firmly rooting this description in the degrees of freedom of the Standard Model seems to be a challenge for the next generation.

$A b$ initio approaches, whose difficulties are sometimes quoted as proof of the ultimate impossibility of computing nuclei with realistic interactions, are showing signs of reinvigoration [Som14] after the initial boost of results produced in the nineties [Pie01]. The modeling of the nuclear interaction itself has discovered a new foundation through chiral effective field theory, a systematic framework for its perturbative expansion and, on the long-term, the most promising link to quantum chromodynamics [Epe09]. Exploiting also renormalization-group methods, this approach cures the long-standing problem of having to deal with numerically inadequate realistic interactions [Bog10]. Constrained entirely on nucleon-nucleon scattering data and the properties of three-nucleon systems (helium-3, tritium), it offers a whole new class of effective interactions for shell-model calculations, which are both predictive and physically consistent. In particular, this framework accounts in a systematic way for the contribution of three-nucleon forces, the role of which, largely disregarded in the past, is again being closely scrutinized [Ots10; Hol12; Gal12; Wie13].

Self-consistent mean-field theory with Skyrme interactions [Ben03], extensively used up to now for calculations, has produced the first mass tables comparably accurate to fits by macroscopic-microscopic formulas [Gor09], but quickly reached what seems to be its accuracy limit at a few hundred keV. Treatment of nuclear collective motion beyond the mean field has definitely proven to be a very promising direction of research [Ben06; Del10], but up to now there seems to be no obvious way to make this approach spectroscopically accurate and extend it to odd nuclei. A different point of view draws inspiration from the advances of atomic and condensed-matter physics and exploits the formalism of density-functional theory [Hoh64; Koh65]. This works on the idea of developing a nuclear energy density functional for many-body calculations, which is a complete change of perspective, avoiding the necessity of accurately modeling the nuclear manybody Hamiltonian and still offering the possibility to constrain the model's parameters directly through nuclear observables [Dob11]. This approach allows a smooth continuation of previous work, given that the binding energy in Skyrme-Hartree-Fock-Bogoliubov theory is an energy density functional. 
With these emerging orientations both in experimental and theoretical physics, the activity of the ISOLTRAP collaboration during the last few years has focused on two primary goals: to extend and refine the available experimental data in "key" regions of the nuclear chart, i.e. regions in which the ground-state observables show sudden deviations from the extrapolated trends, hinting at emerging nuclear-structure phenomena; to develop new techniques and refine the existing ones, enhancing ISOLTRAP's purification capability and opening the door for new studies, in which mass spectrometry assists other types of measurements. The content of the thesis reflects these two research goals. Some of the atomic masses measured in the last three years are presented, analyzed and discussed: the masses of neutron-rich rubidium $\left({ }^{98-100} \mathrm{Rb}\right)$ and neutron-deficient gold $\left({ }^{180,185,188,190,191} \mathrm{Au}\right)$ isotopes. A new type of measurement resulting from the installation of a multi-reflection time-of-flight mass spectrometer at ISOLTRAP in 2010 is also discussed. With excellent mass-resolving power and fast operation, the applications of the new device go beyond its use as a beam purifier, allowing ion-beam analysis [Kre13], precision mass spectrometry [Wie13] and ion detection for resonance-ionization laser spectroscopy [Wol13b; Mar13], successfully complementing the other available techniques and even showing its capability to surpass their performance. The application of the MR-TOF MS to the study of the hyperfine structure of exotic nuclides will be the main point of discussion.

The structure of the thesis reflects the close interplay between the advances of experimental and theoretical knowledge from the beginning of mass spectrometry as a field, briefly summarized above. It also underlines the importance of recognizing the manifestations of nuclear structure through different observables as non-redundant. Although signatures in different quantities are to some extent correlated [Cak10], no single type of measurement can fully reflect a certain nuclear-structure phenomenon and only the sum of complementary data can constitute the basis for its unambiguous interpretation. This idea translates to the experimental approach itself by the fact that the treatment of the exotic nuclides by the different apparatus must be exploited to mutually enhance their performance: a mass spectrometer can be used to purify the nuclide of interest from contaminants, for studying its hyperfine structure through resonance-ionization laser spectroscopy [Mar13]; lasers can exploit the known hyperfine structure of the nuclide of interest to resonantly ionize its different nuclear states and provide isomerically pure beams for mass spectrometry and other techniques [Roo04].

The thesis is organized in four chapters. The first chapter offers an introduction of nuclear observables, with the main focus on the nuclear binding energy. Loosely following the developments which historically lead to the formulation of the first microscopic models, a general expression of the nuclear binding energy is given as a starting point for the discussion. This formula illustrates the main features of its experimental trend with proton and neutron number. The expression is not model-independent, because it refers to concepts (eg. single-particle or correlation energies) which only make sense in the framework of a certain model. It is however generic and meant to justify the intuition 
behind every microscopic model:

- the spherical mean-field and the resulting single-particle basis as a first-order approximation to nuclear structure, only accurate for the so-called magic nuclei;

- the energy resulting from the interaction between these first-order degrees of freedom, leading to correlations, increasingly important when moving away from magic numbers;

- the qualitative difference between even and odd nuclei, making their binding energies fall on two different trends, separated by an energy gap - the odd-even staggering phenomenon.

This intuition is justified by introducing the so-called "mass filters", obtained by applying finite-difference formulas to the nuclear binding energy. The way in which the different components of the binding energy are reflected in the mass filters is then discussed based on illustrative examples, pointing out the typical and atypical features of their trends. Finally, the extended picture of complementary nuclear observables is illustrated for the zirconium isotopes, part of the neutron-rich $A \approx 100$ region of the nuclear chart which is studied in this work through the masses of ${ }^{98-100} \mathrm{Rb}$.

The second chapter describes the Penning-trap technique [Bro86] of ion manipulation and cyclotron-frequency measurement, as it is implemented and used by the ISOLTRAP experiment. The time-of-flight ion-cyclotron-resonance [Gra80], used to measure an ion's cyclotron frequency and determine its mass in connection with a reference measurement, is described and its statistical and systematic errors are outlined. The ion-beam preparation and purification techniques used by ISOLTRAP are presented. Complementary applications of the MR-TOF MS as a beam-analysis tool are justified by illustrative examples. The experiments on neutron-rich rubidium and neutron-deficient gold isotopes are described in detail. Apart from the analysis of the measured data, the chapter presents the search for new isomers by the Penning-trap technique and by hyperfine-structure studies using MR-TOF-enhanced resonance-ionization laser spectroscopy.

The third and fourth chapters constitute a detailed analysis of the new mass data in the framework of Hartree-Fock-Bogoliubov (HFB) theory [Rin00]. The third chapter begins with a review of some of the concepts of the nuclear many-body problem and gives an outline of the HFB framework. The main focus is the HFB description of particlehole (e.g. quadrupole) and particle-particle (pairing) correlations. Their competition is responsible for the evolution of nuclear structure in open-shell nuclei. In particular, shape transitions in the ground states of such nuclei are proposed as a sensitive probe of the balance between the two types of correlations. Because the rubidium and gold isotopes cross two of the most prominent shape-transition regions of the nuclear chart, the measured nuclear data is analyzed with respect to this balance. To this end, HFB calculations are performed using the HFODD code [Dob97; Sch12]. The SLy4 [Cha98] and a delta volume pairing force [Ben03] are used for the particle-hole and particle-particle parts of the interaction, respectively. The last part of the third chapter introduces the 
code and a series of test runs, aimed at establishing the method of computation.

The fourth chapter presents the results obtained for neutron-rich rubidium-zirconium and neutron-deficient gold-thallium nuclei. The ground-state binding energies and charge radii are computed and compared to experiment, highlighting the results which correspond to different local mean-field mininima, oblate or prolate deformed in the quadrupole degree of freedom. The strength of the pairing interaction is varied and the change of the general agreement with experiment is discussed, with respect to the energy balance between the different mean-field minima. Particular attention is given to the way the borders of shape-transition regions change with the strength of the pairing interaction. Finally, the odd-even staggering of mercury isotopes is computed and compared to experiment. The pairing-interaction strength offering the best description of mid-shell nuclei is confronted with the value ensuring the most accurate description of the odd-even-staggering of mercury isotopes. An explanation is given in this framework for the strong odd-even staggering of charge radii along the mercury isotopic chain. 


\section{Chapter 1}

\section{Nuclear observables}

\subsection{Binding energy}

According to the energy-mass equivalence theorem of special relativity, the mass defect $\delta m$ of an atomic nucleus with $Z$ protons, $N$ neutrons and mass $M(Z, N)$ corresponds exactly to the binding energy determined by the interaction of its constituents:

$$
\delta m(Z, N)=Z m_{\pi}+N m_{\nu}-M(Z, N)=-E(Z, N) / c^{2},
$$

where $m_{\pi}$ is the proton mass, $m_{\nu}$ is the neutron mass, $E(Z, N)$ is the nuclear binding energy (taken with negative sign ${ }^{1}$ ), and $c$ is the speed of light.

This means that the inertia of a system of composed particles is sensitive to the internal forces acting between them, a result which contradicts the theorems of classical mechanics and requires replacing the instantaneous action at a distance with a local picture, in which interaction propagates at finite velocity. A direct test of the $E=m c^{2}$ relation, proving it accurate to $0.00004 \%$ in an experiment of radiative neutron-capture (in which the measurements are performed on participants to the same reaction), was reported in [Rai05]. This degree of accuracy, if translated to Eq. (1.1), would represent a difference of maximum $1 \mathrm{keV}$ between the two terms of the equality, for the heaviest of known nuclei. This is below the typical precision of experimental atomic masses of radioactive nuclides and much lower than the mean deviation of model predictions from experiment.

The quantity $E(Z, N)$ is what nuclear theory calculates and will be used throughout this work for the physics discussion, instead of the mass defect or the mass excess. The latter is defined as:

$$
m_{\text {exc }}(Z, N)=M(Z, N)-(Z+N) u,
$$

where $u$ is the unified atomic mass unit, defined as $1 / 12$ of the mass of the carbon isotope

\footnotetext{
${ }^{1}$ The quantity $E(Z, N)$ will always be used with this sign convention.
} 
${ }^{12} \mathrm{C}$. The mass excess (rather than the binding energy) is how experimental data are presented in the atomic mass evaluation (AME) [Wan12], because it allows giving the full relevant mass information, in units of $\mathrm{keV} / \mathrm{c}^{2}$, by using a few-digit number. For consistency with the AME, the experimental information will also be presented using this convention.

The simple existence of nuclear binding reveals the fact that the constituents of the nucleus interact by a strong, at least overall attractive force, which overcomes the repulsive Coulomb interaction between the protons. It however does not allow drawing any conclusion about the dynamics of these constituents, the resulting structure or the relevant degrees of freedom for describing it.

In quantum-mechanical terms, the binding energy $E(Z, N)$ is the lowest eigenvalue of the nuclear Hamiltonian. Since the nucleus is a many-body system, it reflects the interaction of all $Z$ protons and $N$ neutrons, meaning that, even in the few cases in which it is computable from realistic interactions, the elementary information contained in $E(Z, N)$ is very difficult to extract. Consequently, the binding energy of a single nucleus can almost never be interpreted on its own and the usual approach is to study the trends of binding energies along the different isotopic or isotonic chains.

It is the interpretation of these trends which historically lead to the formulation of the first nuclear-structure model, the liquid-drop model [Gam30], and of the first nuclearmass formula, the semi-empirical mass formula [Wei35; Bet36]. The formula had a large impact on the subsequent development of nuclear theory, because it soon became the reference with respect to which nuclear-structure effects beyond the simple liquid-drop picture could be revealed.

One such phenomenon, later included in the semi-empirical mass formula, was the odd-even staggering of $E(Z, N)$, referring to the fact that every even- $Z / N$ nucleus is more bound than the average of its odd $Z / N$ neighbors, respectively. Another phenomenon was the enhanced binding of nuclei with $N \approx Z$, known as the Wigner effect [Wig37]. Other deviations of binding energies from the liquid-drop model, showing enhanced nuclear stability for some "magic" values of $Z$ and $N$, were later crucial for the formulation of the first microscopic nuclear model [May49; Hax49]). This added to other existing evidence, including the number, $(Z, N)$ distribution and abundance of stable nuclides, the asymmetry of fission, the energies of alpha decays to the lead isotopes and the neutron absorption and emission probabilities of certain nuclei [May48]. These observations could be at least qualitatively understood if one regarded the nucleus as a system of independent particles orbiting in the average potential created by all the other particles together. Such a potential generates a set of single-particle levels, called subshells, which are occupied according to the Pauli principle. Depending on the shape of the average potential, subshells cluster in groups separated by large energy gaps (of a few $\mathrm{MeV}$ ), called shells. In this picture, nuclei with enhanced stability (magic nuclei) correspond to those numbers of protons and neutrons which lead to the complete filling of a shell, in perfect analogy to atomic physics. 
A strong nuclear spin-orbit interaction was postulated to explain the "standard" magic numbers, which are now believed to be 2, 8, 20, 28, 50, 82, 126.

Similar deviations from the liquid-drop model midway between the generally accepted nuclear shells were later linked to the phenomenon of "configuration mixing" [Hog53]. Contrary to the closure of nuclear shells, in this case the nucleus gains binding energy by forming configurations in which its quantum mechanical state is a superposition of many independent-particle states. For these nuclei the independent-particle approximation breaks down and nuclear structure is the result of the collective motion of many nucleons. Contrary to the independent-particle picture, collective motion means that the dynamics of protons and neutrons is correlated, which leads to the name "correlation energy" for the extra binding gained by configuration mixing. The interplay between independent-particle and collective motion in nuclei is at the heart of all modern microscopic approaches [Ben03; Cau05].

\subsection{Trends of binding energies}

The most recent atomic-mass evaluation, AME2012 [Wan12] has reported a number of 2438 experimentally known masses, which by use of Eq. (1.1) yield as many nuclear binding energies. The basic observations following the first mass measurements, which lead to the early nuclear-structure concepts mentioned in the previous section, are nowadays confirmed by the available data to a remarkable extent throughout the entire explored $(Z, N)$ range of the nuclear chart. Deviations from the expected trends of binding energies are sought through challenging experiments on unstable nuclides, having a great neutron excess or deficit with respect to the stable isotopes. When such deviations are found, they are used to confront state-of-the art theoretical approaches, in search for new intuitions on nuclear structure.

The method of interpreting the mass data is however different. The binding energy is a large quantity (up to $\approx 2 \mathrm{GeV}$ ) and its typical variation with every added proton or neutron is of a few $\mathrm{MeV}$, making it very difficult to study the evolution of the underlying nuclear structure directly with $E(Z, N)$. To illustrate this, Fig. 1.1 shows in the upper panel the known binding energies of the calcium isotopes, using data from AME2012 [Wan12] and the masses of ${ }^{51-54} \mathrm{Ca}$ from a recent publication of the ISOLTRAP experiment [Wie13]. The slight changes of slope at the crossing of the magic numbers $N=20$ and $N=28$, as well as the odd-even staggering effect, are barely visible. In order to bring out these effects, finite-difference operators, involving two or more nuclei, are applied to the binding energies to compute more sensitive quantities. Because these operators are usually meant to isolate from the whole energy the part corresponding to a specific effect, the resulting quantities are called mass filters. 


\subsubsection{Decomposition and extension of the binding energy func- tion}

Before exemplifying some of the typical mass filters, one can introduce a generic formula which explicitly separates the different contributions to the nuclear binding energy $E$ :

$$
\begin{aligned}
E(Z, N) & =E_{0}(Z, N)+E_{1}(Z, N)+\frac{(-1)^{Z+1}+1}{2} \delta_{\pi}(Z, N) \\
& +\frac{(-1)^{N+1}+1}{2} \delta_{\nu}(Z, N) .
\end{aligned}
$$

The decomposition between $E_{0}$ and $E_{1}$ is a priori completely arbitrary and it remains the role of theory to link the two terms through explicit formulas or numeric representations to $Z$ and $N$. The idea of expressing the binding energy as a sum or hierarchy of different contributions has remained an appealing approach since the first liquid-drop formulas [Wei35; Bet36]. The two terms $E_{0}$ and $E_{1}$ can for example represent the liquid-drop (macroscopic) and shell-correction (microscopic) energies of the so-called microscopic-macroscopic approaches, which were formulated in the sixties and seventies [Mye66; Str67] and culminated with the very successful finite-range droplet model [Mol95]. They can be any partition of the different terms appearing in a pure macroscopic approach, such as the liquid droplet model [Mye 74], $E_{0}$ being for instance the spherical part of the binding energy and $E_{1}$ the energy gain obtained by allowing a deformed droplet shape (deformation energy). Pure microscopic approaches can also produce expressions of the binding energy which allow the decomposition of Eq. (1.3). In Hartree-Fock theory [Ben03] the energy is a functional of density with additive character, either between the parts corresponding to different terms of the interaction, or between the result obtained at a certain level of approximation and the subsequent corrections (see Section 3.3.1 for a more detailed discussion on the energy-density functional and Eq. (4.1) for a relation to observables). The extended Thomas-Fermi plus Strutinsky integral approximation to the Hartree-Fock method allows the division between the macroscopic and shell-correction parts of the binding energy starting from an initial interaction [Abo95].

The third term of Eq. (1.3) is invariably referring in these approaches (with the appropriate extensions) to the odd-even staggering of binding energies.

For the following discussion, instead of opting for a specific mass formula corresponding to one of the different models mentioned above, the terms in Eq. (1.3) are defined in a quite general way to illustrate the experimentally observed behavior of nuclear binding energies. Based on this decomposition, one can then study how the different contributions to $E$ are reflected in the mass filters.

Thus, the $E_{0}$ term is considered the part of the binding energy resulting from the best approximation of the $(Z, N)$ nucleus as a system of independent particles orbiting in a spherical potential. This gives the zero-order description of the system, as the one proposed in the works of [May49; Hax49]. The binding energy of the system in an 
independent-particle problem is simply:

$$
E_{0}(Z, N)=\sum_{i=1}^{Z} \epsilon_{\pi, i}(Z, N)+\sum_{j=1}^{N} \epsilon_{\nu, j}(Z, N)
$$

where $\epsilon_{\pi, i}$ and $\epsilon_{\nu, j}$ are the proton and neutron single-particle energies, respectively (negative quantities). The single-particle energies can vary with $Z$ and $N$.

The term $E_{1}$ contains every effect which cannot be simply averaged by an independentparticle potential and thus is related to the inherent correlations between the particles of the system, induced by their interactions (correlation energy). This term includes the Wigner effect, a part which peaks for $N \approx Z$. It also includes correlations which are related to the quantum-mechanical mixing of the single-particle states of the valence particles. The resulting correlation energy depends on the number of valence particles and thus peaks midway between magic numbers, decreasing in strength towards them.

Finally, $\delta_{\pi}$ and $\delta_{\nu}$ describe the odd-even staggering effect of nuclear binding energies as a function of proton and neutron number, and will be called odd-even proton and neutron gaps, respectively (positive quantities).

The observable binding energy $E(Z, N)$ and the four terms of Eq. (1.3) are discrete quantities, only defined for integer $Z$ and $N$. For certain points of the following discussion it will be useful to regard these terms as continuous, infinitely derivable functions, which are equal to the observable values for integer arguments. With this extension one is able to link the action of finite-difference operators, by which mass filters are computed, to the derivatives of the binding-energy components with respect to proton and neutron number. This is a way to test the sensitivity of certain mass filters to the analytical structure of the binding energy, acting as a constraint for models in which the extension to continuous $(Z, N)$ arguments is possible. It is also a way to estimate which of the energy components still effectively contribute to a certain mass filter (a term which is known to be very smooth will be effectively canceled by a mass filter which only retains the high-order derivatives). This is not uncommon practice and for models which give analytical mass formulas it comes naturally, because one is only required to take $Z$ and $N$ as continuous arguments. One such study is the one of Madland and Nix [Mad88], aimed at modeling the pairing gap, for which different-order mass filters are proposed. A study making use of this approach to discuss the pairing gap in mean-field theory was published by Bender et al. [Ben00].

With this extension, one can expand each of the four terms of Eq. (1.3) in powers of the neutron number $N$, around their values at $\left(Z_{0}, N_{0}\right)$ (or similarly in powers of the proton number $Z$ ):

$$
T(Z, N)=\sum_{n=0}^{\infty} \frac{1}{n !}\left(\frac{\partial^{n} T}{\partial N^{n}}\right)_{Z, N=N_{0}}\left(N-N_{0}\right)^{n}
$$


One will not use a special notation for the continuous and observable $E_{0,1}$ and $\delta_{\pi, \nu}$, but the context will clarify which one of the two is concerned.

\subsubsection{Mass filters}

A brief mathematical introduction of the finite-difference operators discussed below and of their action on the different terms of a function's Taylor expansion is provided in the Appendix A. The results derived there for a general function $F(X)$ will be directly translated to the components of $E(Z, N)$.

As mentioned before, the mass filters are obtained by applying a finite-difference operator to the binding energy $E$, leading to a formula which involves two or more nuclei. In the following, we will use different notations for the mass filters and the corresponding finite-difference operators, because the latter can act on any component of $E$, while the concept of mass filter refers to the special case in which they act on the whole binding energy. The following discussion will be divided between estimators of the single-particle energies and estimators of the odd-even staggering effect, including at the end a brief illustration of some of the residual contributions which affect their trends.

\section{Estimators of the single-particle energies}

The simplest mass filter, obtained by acting on $E$ with the $\mathcal{D}_{1}$ finite-difference operator ${ }^{2}$ (see also Appendix A), is the one-nucleon (proton or neutron) separation energy $\left(S_{p}\right.$ and $S_{n}$, respectively):

$$
\begin{aligned}
& S_{p}(Z, N)=\mathcal{D}_{1, Z} E(Z, N)=E(Z-1, N)-E(Z, N), \\
& S_{n}(Z, N)=\mathcal{D}_{1, N} E(Z, N)=E(Z, N-1)-E(Z, N) .
\end{aligned}
$$

Using Eq. (1.3), the expression of $S_{n}$ becomes:

$$
\begin{aligned}
S_{n}(Z, N) & =\sum_{i=1}^{Z}\left[\mathcal{D}_{1, N} \epsilon_{\pi, i}(Z, N)\right]+\sum_{j=1}^{N-1}\left[\mathcal{D}_{1, N} \epsilon_{\nu, j}(Z, N)\right]-\epsilon_{\nu, N}(Z, N) \\
& +\mathcal{D}_{1, N} E_{1}(Z, N)+\frac{(-1)^{Z+1}+1}{2} \mathcal{D}_{1, N} \delta_{\pi}(Z, N) \\
& +\frac{1}{2} \mathcal{D}_{1, N} \delta_{\nu}(Z, N)+\frac{(-1)^{N}}{2}\left[\delta_{\nu}(Z, N-1)+\delta_{\nu}(Z, N)\right] .
\end{aligned}
$$

\footnotetext{
${ }^{2}$ Throughout this work the finite-difference operators will be labeled by using the calligraphic font $\mathcal{D}$, while $\Delta$ will only be used for the physical quantities obtained by applying the operators to the binding energy
} 
Most terms in Eq. (1.8) refer to the variation between $N-1$ and $N$ of the different binding-energy components. The first two terms express the slight change with $N$ of the sum of proton and neutron single-particle energies up to $Z$ and $N-1$, respectively (singleparticle rearrangement energy), which is expected to be small compared to the third term, the energy of the last neutron single-particle state. The terms proportional to $\mathcal{D}_{1, N} \delta_{\pi}$ and $\mathcal{D}_{1, N} \delta_{\nu}$ are only a small fraction of $S_{n}$. The $\mathcal{D}_{1, N} E_{1}$ term is in many cases a small quantity, but in regions of sudden structural changes it can acquire a large value. While the small terms of Eq. (1.7) are very important for understanding the full systematics of $S_{n}$ with $Z$ and $N$, neglecting them in a first approximation brings out the main contributions to the average trend of the $S_{n}$ mass filter:

$$
S_{n}(Z, N) \approx-\epsilon_{\nu, N}(Z, N)+\mathcal{D}_{1, N} E_{1}(Z, N)+(-1)^{N} \bar{\delta}_{\nu}(Z, N),
$$

where $\overline{\delta_{\nu}}$ is the average odd-even neutron gap between $N$ and $N-1$.

The one-neutron separation energies of the calcium isotopes are shown in the second panel of Fig. 1.1. One recognizes two main features: first of all, the staggering of $S_{n}$, resulting from the $\overline{\delta_{\nu}}$ term, which dominates the plot; second of all, the pronounced drop of $S_{n}$ at the crossing of the magic neutron numbers $N_{0}=20$ and 28 , which is related to the energy of the last occupied neutron single-particle level $\epsilon_{\nu, N}$. Indeed, because of the energy degeneracy of single-particle levels, $\epsilon_{\nu, N}$ will have only a small variation with the addition of neutrons, as long as they are filling the same level. When the level is full, however, a new one, higher in energy, starts to be filled and the value of $\epsilon_{\nu, N}$ suddenly changes with the addition of a single neutron, producing the drop in $S_{n}$. The amount of this drop for a magic neutron number $N_{0}$ (also called the one-neutron gap, $\Delta_{1 n}$ ) is:

$$
\begin{aligned}
\Delta_{1 n}\left(Z, N_{0}\right) & =S_{n}\left(Z, N_{0}\right)-S_{n}\left(Z, N_{0}+1\right) \\
& =\mathcal{D}_{1, N} S_{n}\left(Z, N_{0}+1\right)=\left(\mathcal{D}_{1, N}\right)^{2} E\left(Z, N_{0}+1\right),
\end{aligned}
$$

which, using Eq. (1.8), can be written as:

$$
\begin{aligned}
\Delta_{1 n}\left(Z, N_{0}\right) & =\sum_{i=1}^{Z}\left[\left(\mathcal{D}_{1, N}\right)^{2} \epsilon_{\pi, i}\left(Z, N_{0}+1\right)\right]+\sum_{j=1}^{N_{0}-1}\left[\left(\mathcal{D}_{1, N}\right)^{2} \epsilon_{\nu, j}\left(Z, N_{0}+1\right)\right] \\
& -\mathcal{D}_{1, N} \epsilon_{\nu, N_{0}}\left(Z, N_{0}+1\right)+\delta \epsilon_{\nu, N_{0}}(Z) \\
& +\left(\mathcal{D}_{1, N}\right)^{2} E_{1}\left(Z, N_{0}+1\right)+\frac{(-1)^{Z+1}+1}{2}\left(\mathcal{D}_{1, N}\right)^{2} \delta_{\pi}\left(Z, N_{0}+1\right) \\
& +\frac{1}{2}\left(\mathcal{D}_{1, N}\right)^{2} \delta_{\nu}\left(Z, N_{0}+1\right)+2 \bar{\delta}_{\nu}\left(Z, N_{0}\right)
\end{aligned}
$$

where we have taken into account that $N_{0}$ is an even number. The quantity $\delta \epsilon_{\nu, N_{0}}$ is the energy gap above the shell which fills at $N=N_{0}$ in the single-particle picture (the "real" 


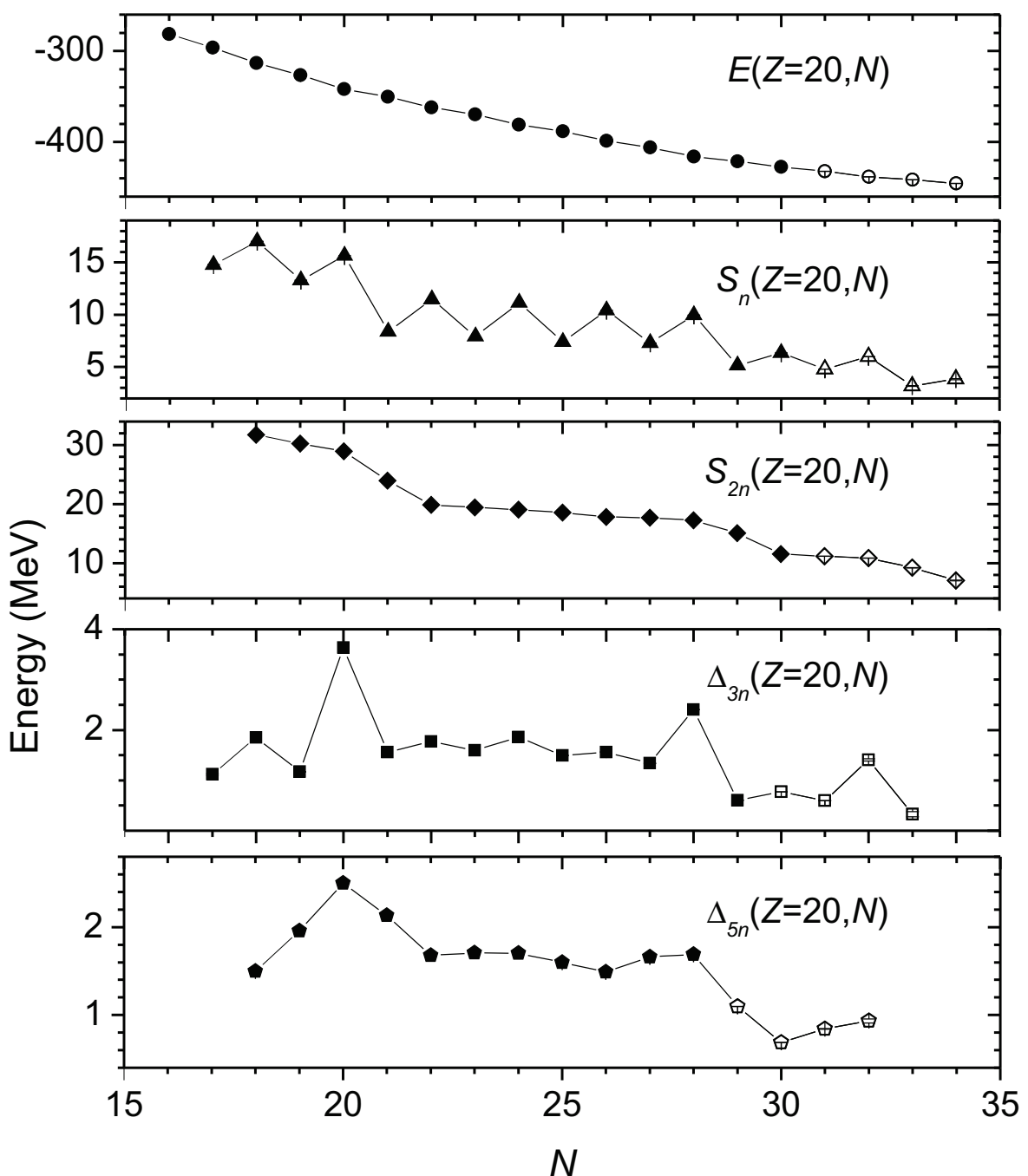

Figure 1.1: Experimental binding energies and different mass filters for the calcium isotopes, based on the data from the AME2012 [Wan12] (full symbols) and recent ISOLTRAP masses of ${ }^{51-54} \mathrm{Ca}$ [Wie13] (open symbols). The ISOLTRAP masses of ${ }^{51,52} \mathrm{Ca}$ replace the values of AME2012, which have as main contribution the Penning-trap measurements performed at TRIUMF with the TITAN experiment [Gal12]. For details, see text.

shell gap). One observes that $\Delta_{1 n}\left(Z, N_{0}\right)$ contains several terms, including a staggering with respect to proton number by $\left(\mathcal{D}_{1, N}\right)^{2} \delta_{\pi}$. As in the case of $S_{n}$, neglecting the terms which contain the action of the $\mathcal{D}_{1, N}$ filter on slow-varying quantities, the average one- 
neutron gap is given in the first approximation by:

$$
\Delta_{1 n}\left(Z, N_{0}\right) \approx \delta \epsilon_{\nu, N_{0}}(Z)+\left(\mathcal{D}_{1, N}\right)^{2} E_{1}\left(Z, N_{0}+1\right)+2 \overline{\delta_{\nu}}\left(Z, N_{0}\right)
$$

Typically a few MeV large, $\delta \epsilon_{\nu, N_{0}}$ gives the leading contribution to $\Delta_{1 n}$, which makes this mass filter adequate for studying nuclear magicity and its link to the underlying single-particle picture. There is however a significant contribution from the neutron oddeven staggering $\delta_{\nu}$, as well as from the correlation energy $E_{1}$. Especially in mid-shell regions or in the case of $N \approx Z$ nuclei, sudden local variations of $E_{1}$ can occur. This term contributes, for example, to $\Delta_{1 n}(20,20)$ through the Wigner effect, which makes in Fig. 1.1 that the drop of $S_{n}$ observed after $N_{0}=20$ is very pronounced.

Using now the Taylor decomposition of $E_{1}$ around $N_{0}$ given by Eq. (1.5) and the result of Eq. (A.8), one can write Eq. (1.12) as:

$$
\Delta_{1 n}\left(Z, N_{0}\right) \approx \delta \epsilon_{\nu, N_{0}}(Z)+\left(\frac{\partial^{2} E_{1}}{\partial N^{2}}\right)_{N_{0}}+\frac{1}{12}\left(\frac{\partial^{4} E_{1}}{\partial N^{4}}\right)_{N_{0}}+\ldots+2 \bar{\delta}_{\nu}\left(Z, N_{0}\right),
$$

which shows that the one-neutron gap is affected by the even-order derivatives of $E_{1}$ with respect to $N$, of which the dominating one is the second derivative. The Taylor expansion can be identically applied to the other terms of Eq. (1.11).

A similar discussion can also be made for the one-proton separation energy and oneproton gap. The resulting formulas are easy to obtain by interchanging arguments directly in Eqs. (1.8) to (1.13).

The disadvantage of applying the $\mathcal{D}_{1}$ finite-difference operator is that the result mixes the odd-even staggering with the single-particle picture. The contribution of the odd-even staggering can be reduced by taking differences of binding energies of nuclei differing by two protons or neutrons ( $\mathcal{D}_{2}$ finite-difference operator, see also Appendix A), which gives the two-proton or two-neutron separation energies $\left(S_{2 p}\right.$ and $S_{2 n}$, respectively):

$$
\begin{aligned}
S_{2 p}(Z, N) & =\mathcal{D}_{2, Z} E(Z, N)=\mathcal{D}_{1, Z}[E(Z-1, N)+E(Z, N)] \\
& =E(Z-2, N)-E(Z, N), \\
S_{2 n}(Z, N) & =\mathcal{D}_{2, N} E(Z, N)=\mathcal{D}_{1, N}[E(Z, N-1)+E(Z, N)] \\
& =E(Z, N-2)-E(Z, N) .
\end{aligned}
$$

Calculating the two-nucleon separation energy is thus equivalent to applying $\mathcal{D}_{1, N}$ to the sum of the binding energies of two adjacent nuclei, or to calculating the sum of their single-nucleon separation energies.

Using again the decomposition of the binding energy given in Eq. (1.3), one arrives at the expression: 


$$
\begin{aligned}
S_{2 n}(Z, N) & =\sum_{i=1}^{Z}\left[\mathcal{D}_{2, N} \epsilon_{\pi, i}(Z, N)\right]+\sum_{j=1}^{N-2}\left[\mathcal{D}_{2, N} \epsilon_{\nu, j}(Z, N)\right] \\
& +\mathcal{D}_{2, N} E_{1}(Z, N)-\epsilon_{\nu, N}(Z, N)-\epsilon_{\nu, N-1}(Z, N) \\
& +\frac{(-1)^{Z+1}+1}{2} \mathcal{D}_{2, N} \delta_{\pi}(Z, N)+\frac{(-1)^{N+1}+1}{2} \mathcal{D}_{2, N} \delta_{\nu}(Z, N) .
\end{aligned}
$$

One notices that $S_{2 n}$ has a similar expression to $S_{n}$, but this time the single-particle energies of the last two neutrons $\epsilon_{\nu, N}, \epsilon_{\nu, N-1}$ appear explicitly. The odd-even staggering with neutron number $\delta_{\nu}$ is acted on by the $\mathcal{D}_{2, N}$ operator, meaning that only its variation contributes to the two-neutron separation energy. Neglecting in the first approximation the terms of Eq. (1.16) which contain the action of $\mathcal{D}_{2}$ on slow-varying quantities, the first-order contributions to the average $S_{2 n}$ trend are:

$$
S_{2 n}(Z, N) \approx-\epsilon_{\nu, N}(Z, N)-\epsilon_{\nu, N-1}(Z, N)+\mathcal{D}_{2, N} E_{1}(Z, N)
$$

where again we have taken into account the possibility of sudden changes of the correlation energy $E_{1}$ from one neutron number to the next.

The two-neutron separation energies of the calcium isotopes are shown in the third panel of Fig. 1.1. The contribution of the odd-even staggering is greatly suppressed. What characterizes now the trend of $S_{2 n}$ is the two-step drop after the crossing of the neutron magic numbers $N=20$ and 28. As discussed in the case of $S_{n}$, while neutrons are added to the same single-particle level, $\epsilon_{\nu, N}$ and $\epsilon_{\nu, N-1}$ have only a weak variation with $N$ and thus there is no significant change in the trend of two-neutron separation energies. When a neutron shell is full and the first neutron is placed on a higher single-particle level (e.g. at $N=21$ ), the term $\epsilon_{\nu, N}$ suffers a sudden increase, while $\epsilon_{\nu, N-1}$ remains almost unchanged. This leads to the first step of the decrease of $S_{2 n}$. When one more neutron is added on the higher-energy single-particle level (e.g. at $N=22$ ), the term $\epsilon_{\nu, N-1}$ also increases suddenly, leading to the second step of the decrease of $S_{2 n}$.

The amount by which $S_{2 n}$ drops between a magic number $N_{0}$ and $N_{0}+2$ is called the two-neutron gap $\Delta_{2 n}$ and is defined as:

$$
\begin{aligned}
\Delta_{2 n}\left(Z, N_{0}\right) & =S_{2 n}\left(Z, N_{0}\right)-S_{2 n}\left(Z, N_{0}+2\right) \\
& =\mathcal{D}_{2, N} S_{2 n}\left(Z, N_{0}+2\right),
\end{aligned}
$$

which, using Eq. (1.16), is written as:

$$
\begin{aligned}
\Delta_{2 n}\left(Z, N_{0}\right) & =\sum_{i=1}^{Z}\left[\left(\mathcal{D}_{2, N}\right)^{2} \epsilon_{\pi, i}\left(Z, N_{0}+2\right)\right]+\sum_{j=1}^{N_{0}-2}\left[\left(\mathcal{D}_{2, N}\right)^{2} \epsilon_{\nu, j}\left(Z, N_{0}+2\right)\right] \\
& +2 \delta \epsilon_{\nu, N_{0}}(Z)-\mathcal{D}_{2, N} \epsilon_{\nu, N_{0}-1}\left(Z, N_{0}+2\right)-\mathcal{D}_{2, N} \epsilon_{\nu, N_{0}}\left(Z, N_{0}+2\right) \\
& +\frac{(-1)^{Z+1}+1}{2}\left(\mathcal{D}_{2, N}\right)^{2} \delta_{\pi}\left(Z, N_{0}+2\right)+\left(\mathcal{D}_{2, N}\right)^{2} E_{1}\left(Z, N_{0}+2\right) .
\end{aligned}
$$


The expression of the two-neutron gap does not contain anymore $\delta_{\nu}$, but it still exhibits a staggering with respect to proton number by the quantity $\left(\mathcal{D}_{2, N}\right)^{2} \delta_{\pi}$. Except for the correlation energy $E_{1}$, all terms of Eq. (1.19) are expected to have a slow variation with neutron number, meaning that the average trend of the two-neutron gap is in the first approximation given by:

$$
\Delta_{2 n}\left(Z, N_{0}\right) \approx 2 \delta \epsilon_{\nu, N_{0}}(Z)+\left(\mathcal{D}_{2, N}\right)^{2} E_{1}\left(Z, N_{0}+2\right) .
$$

As in the case of $\Delta_{1 n}$, the correlation energy $E_{1}$ can have a significant contribution to $\Delta_{2 n}$ especially in mid-shell and $Z \approx N$ regions. In the case of $N=20, \Delta_{2 n}$ and the related drop of $S_{2 n}$ contain a contribution from the Wigner effect through the term $\left(\mathcal{D}_{2, N}\right)^{2} E_{1}$. If one uses again the Taylor expansion of $E_{1}$ around $N_{0}$ and the result of Eq. (A.12), Eq. (1.18) can be written as:

$$
\Delta_{2 n}\left(Z, N_{0}\right) \approx 2 \delta \epsilon_{\nu, N_{0}}(Z)+4\left(\frac{\partial^{2} E_{1}}{\partial N^{2}}\right)_{N_{0}}+\frac{4}{3}\left(\frac{\partial^{4} E_{1}}{\partial N^{4}}\right)_{N_{0}}+\ldots
$$

which shows that, as $\Delta_{1 n}$, the two-neutron gap is also affected by the correlation energy $E_{1}$ through its even-order derivatives, of which the second derivative has the largest weight.

Expressions for the two-proton separation energy and two-proton gap can be directly obtained by interchanging the arguments in Eqs. (1.16) to (1.21).

Equations (1.9), (1.12), (1.17) and (1.20) show that the mass filters discussed so far are adequate for studying shell effects, due to their sensitivity to the gaps in the associated single-particle spectrum. One example is the $N_{0}=32$ effect in the two-neutron separation energies of the calcium isotopes shown in the third panel of Fig. 1.1. The ISOLTRAP measurements of the masses of ${ }^{51-54} \mathrm{Ca}$ revealed a significant two-neutron gap at $N_{0}=32$, which was interpreted as the result of the closure of the $N_{0}=32$ subshell, providing a new important benchmark for theory [Wie13]. Still, they are not perfect estimators of the single-particle energies and corresponding gaps, due to their sensitivity to the correlation energy and to the odd-even staggering effect.

The two-nucleon separation energy can also be used to study the development of nuclear collectivity because Eq. (1.17) is sensitive to the correlation energy $E_{1}$. Between magic numbers the variation of $E_{0}$ is smooth, thus sudden changes in $E_{1}$ become visible as departures from the smooth $S_{2 n}$ or $S_{2 p}$ trend. A well-known example is that of the neutron rich $A \approx 100$ nuclei, for which the two-neutron separation energies [Wan12; Man13] are plotted in Fig. 1.2.

Along the krypton isotopic chain $(Z=36), S_{2 n}$ varies smoothly, showing no significant departure from the trend of the first isotopes with $N>52$. This does not mean that the term $E_{1}$ does not vary much between $N=52$ and $N=61$, but only that the amount by which it varies from one neutron number to the next stays approximately the same. For the rubidium $(Z=37)$ isotopic chain, however, $S_{2 n}$ shows a significant increase at 
$N=61$, a similar phenomenon taking place in the isotopic chains between strontium $(Z=38)$ and molybdenum $(Z=42)$, visible already at $N=60$. This means that the correlation energy $E_{1}$ suddenly increases in absolute value between $N=59$ and $N=61$. The term $\mathcal{D}_{2, N} E_{1}$ of Eq. (1.17) is significantly higher at $N=60,61$ than at $N=59,60$, respectively, which leads to an increase in $S_{2 n}$. We note that usually one observes only a flattening of the $S_{2 n}$ trend due to the correlation energy, very large changes of $E_{1}$ from one neutron to the next being required to obtain a locally positive $S_{2 n}$ slope.

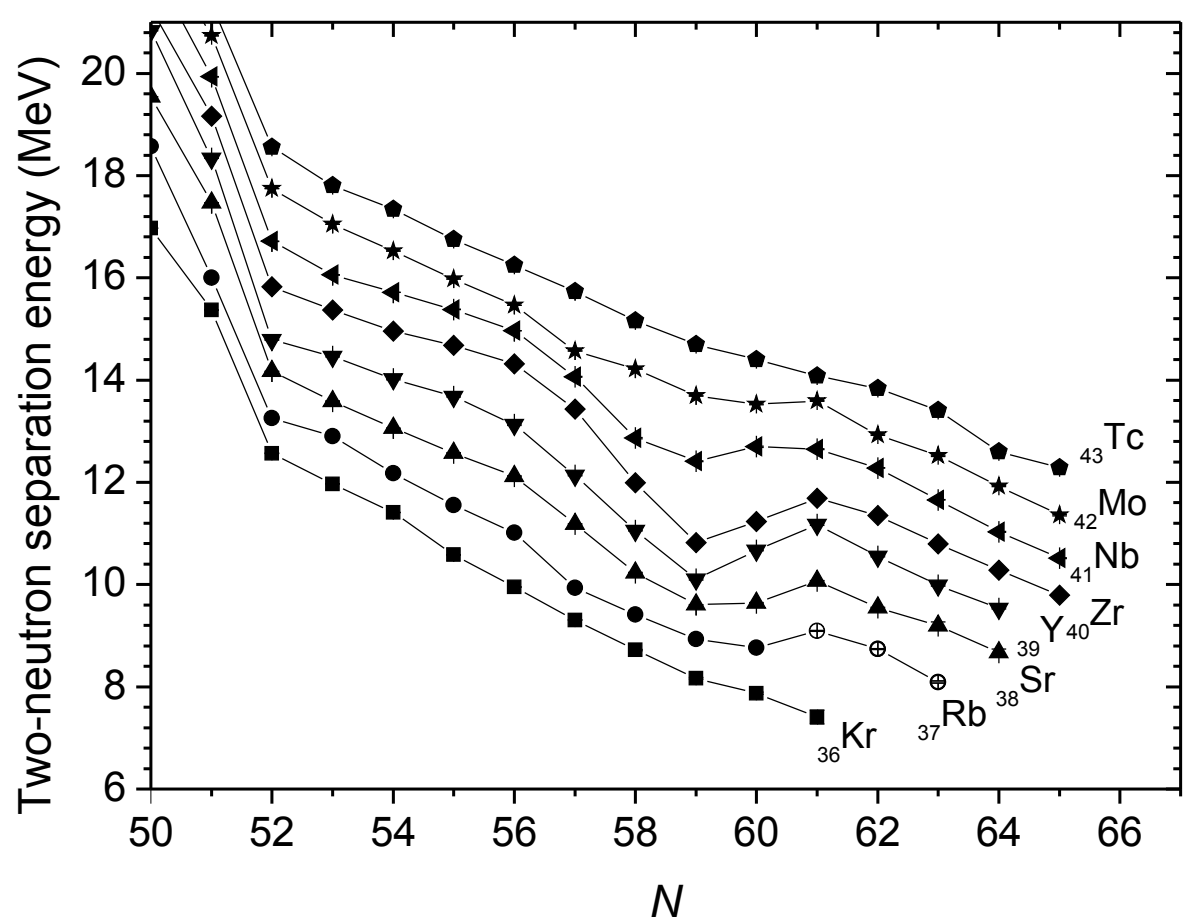

Figure 1.2: Experimental two-neutron separation energies of neutron-rich $A \approx 100$ nuclei from the AME2012 [Wan12] (full symbols) and recent ISOLTRAP masses of ${ }^{98-100} \mathrm{Rb}$ [Man13] (open symbols). The ISOLTRAP masses of ${ }^{98,99} \mathrm{Rb}$ replace the values from the AME2012, which have as main contribution the ${ }^{98} \mathrm{Rb}$ value from [Sim12] and ${ }^{99} \mathrm{Rb}$ value from [Aud86].

\section{Estimators of the odd-even staggering}

The odd-even staggering of binding energies already appears in the expression of the onenucleon gap, as shown in Eq. (1.12), however, if $N$ is not necessarily an even number, the 
expression of $\Delta_{1 n}$ is:

$$
\begin{aligned}
\Delta_{1 n}(Z, N) & \approx \mathcal{D}_{1, N}\left[-\epsilon_{\nu, N+1}(Z, N+1)\right] \\
& +\left(\mathcal{D}_{1, N}\right)^{2} E_{1}(Z, N+1)+2(-1)^{N} \bar{\delta}_{\nu}(Z, N),
\end{aligned}
$$

which shows that $\Delta_{1 n}$ staggers by the quantity $\bar{\delta}_{\nu}(Z, N)$. The lowest-order mass filter which allows estimating the strength of the odd-even staggering follows from Eq. (1.22) and is defined as:

$$
\begin{aligned}
\Delta_{3 n}(Z, N) & =\frac{(-1)^{N}}{2} \mathcal{D}_{1, N}^{2} E(Z, N+1)=\frac{(-1)^{N}}{2} \Delta_{1 n}(Z, N) \\
& =\frac{(-1)^{N}}{2}[E(Z, N-1)-2 E(Z, N)+E(Z, N+1)],
\end{aligned}
$$

which, together with Eq. (1.22), is in the first approximation:

$$
\begin{aligned}
\Delta_{3 n}(Z, N) & \approx \frac{(-1)^{N}}{2} \mathcal{D}_{1, N}\left[-\epsilon_{\nu, N+1}(Z, N+1)\right]+\frac{(-1)^{N}}{2}\left(\mathcal{D}_{1, N}\right)^{2} E_{1}(Z, N+1) \\
& +\bar{\delta}_{\nu}(Z, N) .
\end{aligned}
$$

One notes that the operator $\mathcal{D}_{1, N}$ in the first term of the previous equation does not only act on the $(Z, N)$ argument, but also on the index of the single-particle energy, marked by the square brackets. The name $\Delta_{3 n}$ is not consistent with the way the onenucleon and two-nucleon gaps were defined. For quantities which estimate the odd-even staggering, the index gives the number of different nuclei involved in the formula (three, in the case of $\left.\Delta_{3 n}\right)$.

In Eq. (1.24) one observes that $\Delta_{3 n}$ is approximately equal to the odd-even staggering $\overline{\delta_{\nu}}(Z, N)$, but staggers symmetrical around the values of this quantity. Using the Taylor expansion of $E_{1}(Z, N)$ given by Eq. (1.5) and the result of Eq. (A.8), Eq. (1.24) becomes:

$$
\begin{aligned}
\Delta_{3 n}(Z, N) & \approx \frac{(-1)^{N}}{2} \mathcal{D}_{1, N}\left[-\epsilon_{\nu, N+1}(Z, N+1)\right] \\
& +\frac{(-1)^{N}}{2}\left[\left(\frac{\partial^{2} E_{1}}{\partial N^{2}}\right)_{N}+\frac{1}{12}\left(\frac{\partial^{4} E_{1}}{\partial N^{4}}\right)_{N}+\ldots\right]+\bar{\delta}_{\nu}(Z, N),
\end{aligned}
$$

which shows that the even-order derivatives of $E_{1}(Z, N)$ contribute to $\Delta_{3 n}$, the second derivative having the largest weight. The $\Delta_{3 n}$ values of the calcium isotopes are shown in the fourth panel of Fig. 1.1. One notices clearly the staggering of $\Delta_{3 n}$, due the fact that the first two terms of Eq. (1.24), originating from the single-particle and correlation energy, are not completely suppressed by the action of the $\mathcal{D}_{1, N}$ and $\left(\mathcal{D}_{1, N}\right)^{2}$ operators, respectively. At the crossing of the magic neutron numbers $N=20,28$ (and even $N=32$ ), 
the first term of Eq. (1.24) becomes equal to the shell gap $\delta \epsilon_{\nu, N_{0}}(Z)$ and locally increases the staggering of $\Delta_{3 n}$.

A better estimation of the odd-even staggering requires a higher-order mass filter., i.e. applying the $\mathcal{D}_{1, N}$ operator a greater number of times to the binding energy. The secondorder mass filter is $\Delta_{3 n}$ (see also Appendix A). Applying $\mathcal{D}_{1, N}$ three times to $E(Z, N)$ leads to an asymmetric formula in $N$, involving four nuclei. A symmetric formula is the one corresponding to the fourth power of $\mathcal{D}_{1, N}$ :

$$
\begin{aligned}
\Delta_{5 n}(Z, N) & =\frac{(-1)^{N+1}}{8} \mathcal{D}_{1, N}^{4} E(Z, N+2) \\
& =\frac{(-1)^{N+1}}{8}[E(Z, N-2)-4 E(Z, N-1)+6 E(Z, N) \\
& -4 E(Z, N+1)+E(Z, N+2)],
\end{aligned}
$$

which, using Eq. (1.3) and neglecting the terms which contain the action of differential operators on slow-varying quantities, becomes:

$$
\begin{aligned}
\Delta_{5 n}(Z, N) & \approx \frac{(-1)^{N+1}}{8}\left(\mathcal{D}_{1, N}\right)^{3}\left[-\epsilon_{\nu, N+2}(Z, N+2)\right] \\
& +\frac{(-1)^{N+1}}{8}\left(\mathcal{D}_{1, N}\right)^{4} E_{1}(Z, N+2)+\bar{\delta}_{\nu}(Z, N) .
\end{aligned}
$$

Using also the Taylor expansion of $E_{1}(Z, N)$ from Eq. (1.5) and the result of Eq. (A.10), $\Delta_{5 n}$ can be written as:

$$
\begin{aligned}
\Delta_{5 n}(Z, N) & \approx \frac{(-1)^{N+1}}{8}\left(\mathcal{D}_{1, N}\right)^{3}\left[-\epsilon_{\nu, N+2}(Z, N+2)\right] \\
& +\frac{(-1)^{N+1}}{8}\left[\left(\frac{\partial^{4} E_{1}}{\partial N^{4}}\right)_{N}+\frac{1}{6}\left(\frac{\partial^{6} E_{1}}{\partial N^{6}}\right)_{N}+\ldots\right]+\overline{\delta_{\nu}}(Z, N) .
\end{aligned}
$$

One notes again that in the first term of Eqs. (1.27) and (1.28), the $\left(\mathcal{D}_{1, N}\right)^{3}$ operator acts not only on the $(Z, N)$ argument, but also on the index of the single-particle energy. The values of the $\Delta_{5 n}$ mass filter are shown in Fig. 1.1 for the calcium isotopic chain. The finite-difference formulas $\left(\mathcal{D}_{1, N}\right)^{3}$ and $\left(\mathcal{D}_{1, N}\right)^{4}$ suppress much of the first and second terms of Eq. (1.27), leaving, as Eq. (1.27) shows, only the high-order derivatives. Only sudden changes of the two terms, as the ones which occur at the crossing of a magic neutron number or with the sudden change of correlation energy, respectively, can produce visible effects in $\Delta_{5 n}$. The first case is observed in Fig. 1.1, where the values of $\Delta_{5 n}$ close to $N_{0}=20$ and $N_{0}=28\left(N_{0} \pm 2\right)$ no longer reflect solely the evolution of $\delta_{\nu}(Z, N)$. One notes again that, in the case of $N_{0}=20$, the second term of Eq. (1.27) also contributes due to the Wigner effect, making that the $N_{0}=20$ peak in $\Delta_{5 n}$ is more pronounced than the $N_{0}=28$ one. 
The suppression of the binding-energy derivatives up to the fourth order gives $\Delta_{5 n}$ its smooth trend and recommends it for studying the average evolution of the odd-even staggering of binding energies $\delta_{\nu}(Z, N)$. One notes however that the term $\bar{\delta}_{\nu}(Z, N)$ in Eq. (1.27) represents an average over more nuclides (five) than the similar term of Eq. (1.24) (only three), meaning that $\Delta_{3 n}$ is more sensitive to local effects than $\Delta_{5 n}$.

\section{Residual effects}

In the preceding discussion on mass filters we have presented a series of estimators appropriate to study the nuclear single-particle picture and the odd-even gap and illustrated their sensitivity to other effects than the ones which they are designed to select, producing in some situations obvious signatures. In the following, we will illustrate how the reduction of the experimental uncertainties of atomic masses allows revealing these extra contributions even for mass filters which suppress them to a great extent. It is thus possible to observe the residual contribution of the odd-even staggering to $S_{2 n}, S_{2 p}$ and even $\Delta_{2 n}$, as well as the sensitivity of the one-neutron and two-neutron gaps to the slight augmentation of the correlation energy $E_{1}$ only two nucleons away from magic numbers.

In Eq. (1.16), the staggering of $S_{2 n}$ with proton and neutron numbers is expressed by the last two terms, proportional to $\delta_{2, N} \delta_{\pi}$ and $\delta_{2, N} \delta_{\nu}$, respectively. This means that if the odd-even gap varies strongly with proton or neutron number, the staggering of $S_{2 n}$ with respect to $Z$ or $N$ can become pronounced. To illustrate the possibility of $S_{2 n}$ staggering with respect to $N$, in Fig. 1.3 are represented the $S_{2 n}$ and $\Delta_{3 n}$ values of bismuth $(Z=83)$ and polonium $(Z=84)$ isotopes with $N>126$. In the case of polonium, the odd-even neutron gap, represented by $\Delta_{3 n}$, only increases slightly with neutron number and $S_{2 n}$ has almost no visible staggering. In the case of bismuth, however, the slope of $\Delta_{3 n}$ is significantly higher and the staggering of $S_{2 n}$ is clearly visible.

A similar discussion can be made for the two-proton separation energies, which, taking into account the decomposition of the binding energy of Eq. (1.3), can be written as:

$$
\begin{aligned}
S_{2 p}(Z, N) & =\sum_{i=1}^{Z-2}\left[\mathcal{D}_{2, Z} \epsilon_{\pi, i}(Z, N)\right]+\sum_{j=1}^{N}\left[\mathcal{D}_{2, Z} \epsilon_{\nu, j}(Z, N)\right] \\
& +\mathcal{D}_{2, Z} E_{1}(Z, N)-\epsilon_{\pi, Z}(Z, N)-\epsilon_{\pi, Z-1}(Z, N) \\
& +\frac{(-1)^{Z+1}+1}{2} \mathcal{D}_{2, Z} \delta_{\pi}(Z, N)+\frac{(-1)^{N+1}+1}{2} \mathcal{D}_{2, Z} \delta_{\nu}(Z, N) .
\end{aligned}
$$

Equation (1.29) contains, similarly to Eq. (1.16), the two terms which express the residual staggering of $S_{2 p}$ with respect to proton and neutron number. The last term of Eq. (1.29), proportional to $\mathcal{D}_{2, Z} \delta_{\nu}$, shows that the staggering of $S_{2 p}$ with respect to $N$ is enhanced if the odd-even neutron gap $\delta_{\nu}$ varies significantly between $Z$ and $Z-2$. To illustrate this situation, Fig. 1.4 shows the $S_{2 p}$ values of thallium $(Z=81)$ isotopes, 


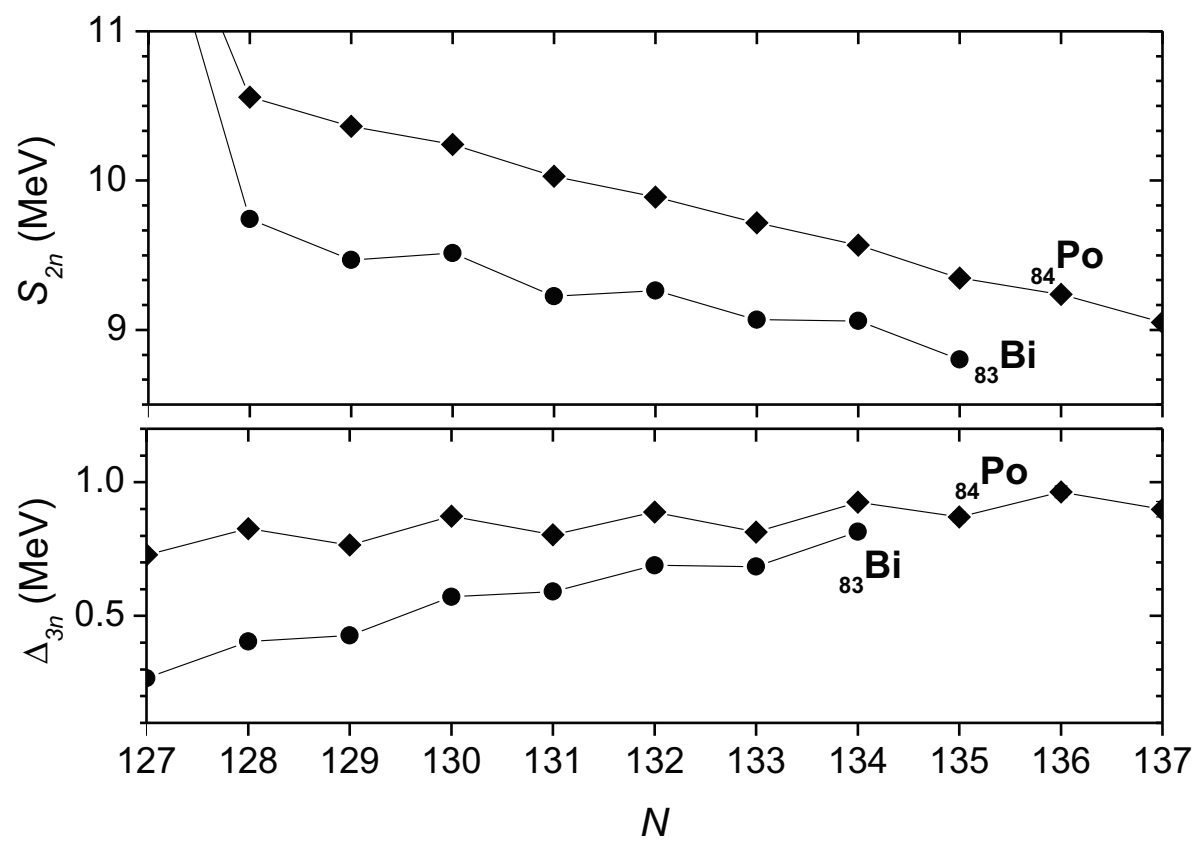

Figure 1.3: Experimental two-neutron separation energies $S_{2 n}$ and three-point estimator of the odd-even neutron gap $\Delta_{3 n}$ for the bismuth $(Z=83)$ and polonium $(Z=84)$ isotopes, using data from the AME2012 [Wan12]. One notices that the high slope of the odd-even neutron gap with neutron number leads to a staggering of $S_{2 n}$.

as well as the $\Delta_{3 n}$ values of thallium and gold $(Z=79)$ isotopes, approximating the odd-even neutron gap. One observes that, in the region in which $\Delta_{3 n}$ is almost the same for thallium and gold, the trend of $S_{2 p}$ with neutron number is relatively smooth. For $99<N<109$, however, the odd-even neutron gap differs significantly between the two chains and in the same region a strong staggering of $S_{2 p}$ occurs.

As observed in Eqs. (1.11) and (1.19), residual staggering with respect to proton number is present also in the one-neutron and two-neutron gaps, although their average trends are not affected by it. The two mass filters are plotted in Fig. 1.5 for the $N=50$ isotones, the two-neutron gap being represented as $\Delta_{2 n} / 2$, because, as shown in Eq. (1.19), it contains twice the gap in the single-particle picture $\delta \epsilon_{\nu, N_{0}}$. The staggering effect is clearly visible in Fig. 1.5, being more pronounced for $\Delta_{1 n}$. The one-neutron gap is also higher, mainly because of the term $2 \bar{\delta}_{\nu}$ appearing in Eqs. (1.11) and (1.12). Both $\Delta_{1 n}$ and $\Delta_{2 n} / 2$ are modified with respect to the gap in the single-particle picture by the even-order derivatives of the correlation energy $E_{1}$, although the fact is not obvious in Fig. 1.5. Perhaps the most clear indication comes from the enhancement towards $Z=28$ of the $\Delta_{1 n}$ and $\Delta_{2 n} / 2$ values, looking at the even- $Z$ isotones. The open-symbol data point is the $\Delta_{2 n} / 2$ value obtained using the new mass of ${ }^{82} \mathrm{Zn}$ determined with ISOLTRAP. Considering the 


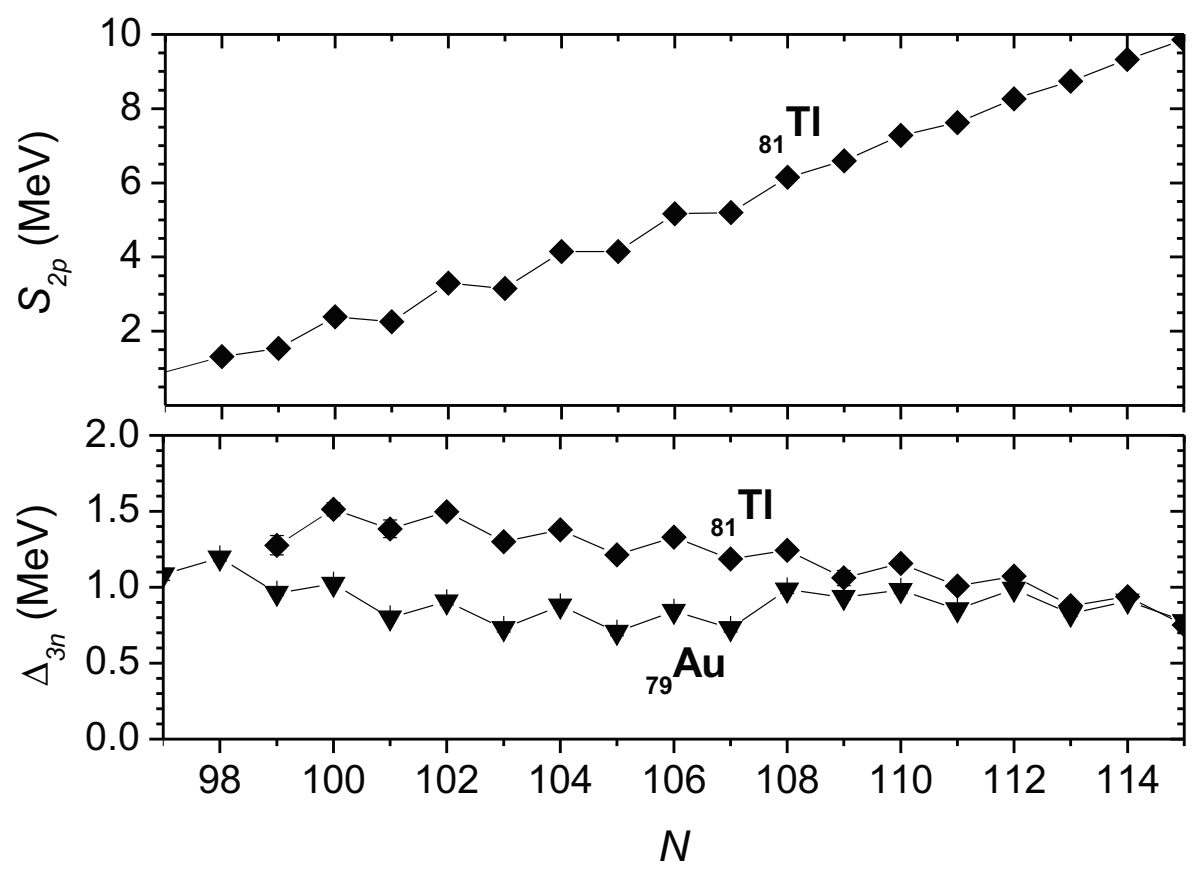

Figure 1.4: Experimental two-proton separation energies $S_{2 p}$ of the thallium $(Z=81)$ isotopes and three-point estimator of the odd-even neutron gap $\Delta_{3 n}$ for the thallium and gold $(Z=79)$ isotopes, using data from the AME2012 [Wan12]. One notices that in the region in which $\Delta_{3 n}$ varies significantly between the thallium and gold isotopic chains, $S_{2 p}$ has a large staggering with neutron number.

odd-even staggering of $\Delta_{2 n}$, it is the first data point showing an enhancement of the twoneutron gap towards $Z=28$. The proton numbers $Z=28,40$ and 50 correspond to closed proton configurations and the correlation energy $E_{1}$ of the $N_{0} \pm 1$ and $N_{0} \pm 2$ isotones is maximum midway between them. Through the terms $\left(\mathcal{D}_{1, N}\right)^{2} E_{1}$ and $\left(\mathcal{D}_{2, N}\right)^{2} E_{1}$ this creates a decrease of the one-neutron and two-neutron gaps, respectively, with respect to $\delta \epsilon_{\nu, N_{0}}$. Towards the magic proton numbers, the reduction of the correlation energy (in absolute value) in the neighboring isotonic chains determines an increase of the two mass filters, which should not be interpreted as an enhancement of the $\delta \epsilon_{\nu, N_{0}}$ gap itself.

\subsection{Complementary nuclear data}

The interpretation of the trends of binding energies through the different mass filters alone can be ambiguous, because different nuclear-structure effects can determine similar features of these trends. For example, a drop in $S_{2 n}$ can be due to the crossing of a magic neutron number, or to a sudden decrease of the absolute value of the correlation energy 


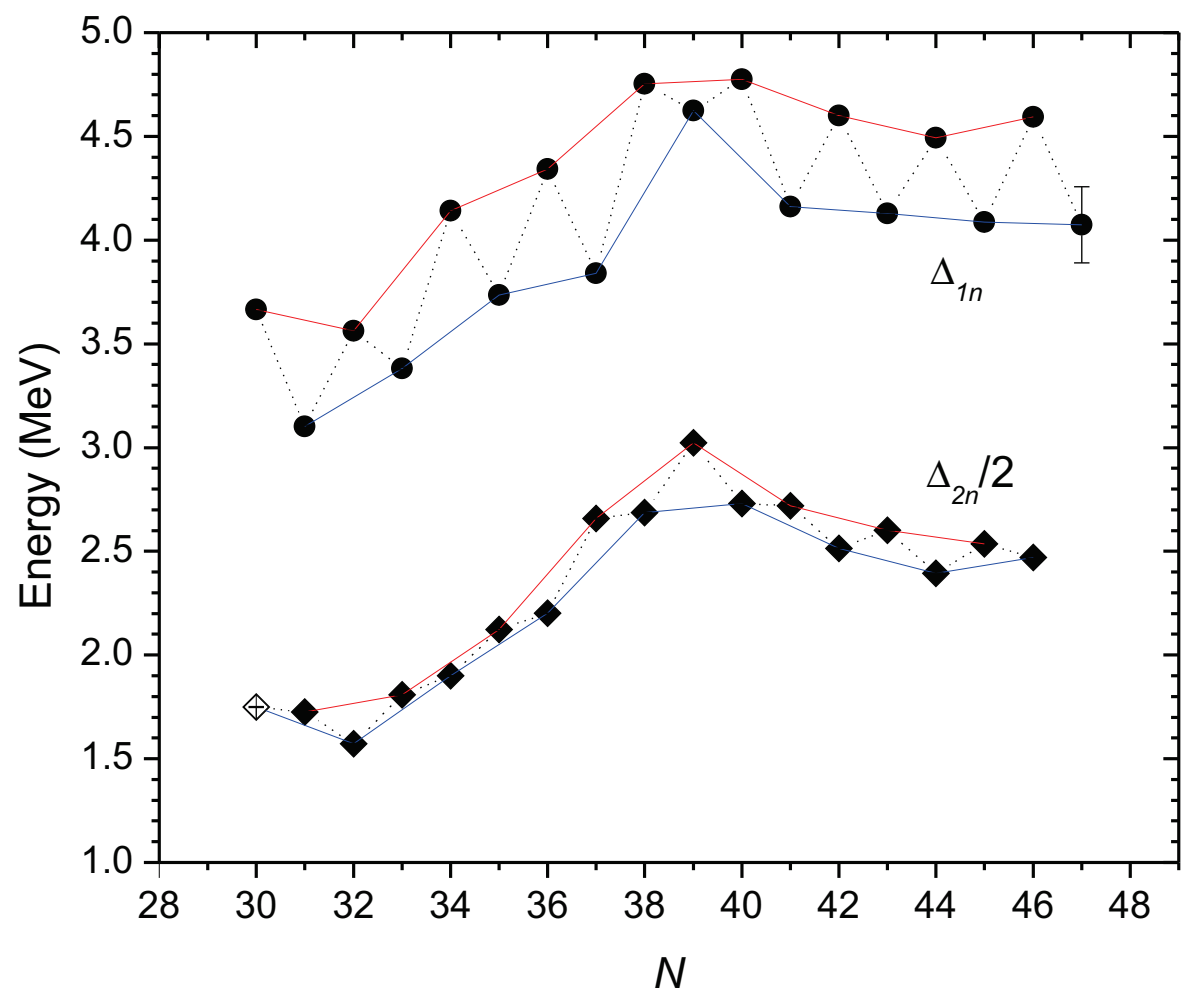

Figure 1.5: Experimental $N=50$ one-neutron and two-neutron gaps ( $\Delta_{1 n}$ and $\Delta_{2 n}$, respectively) as a function of $Z$, using data from the AME2012 [Wan12] (full symbols)). The new two-neutron gap value at $Z=30$, resulting from the mass measurement of ${ }^{82} \mathrm{Zn}$ with ISOLTRAP [Wol13a], is marked by an open symbol. The odd- $Z$ and even- $Z$ values of the two mass filters are connected by continuous lines for guiding the eye.

$E_{1}$ because of a change of nuclear configuration. A gradual change of $\Delta_{2 n}$ can be due to a change of the corresponding gap in the single-particle picture, or of the correlation energy. It is also difficult to trace from simple binding-energy considerations what is the microscopic cause of its features or what type of correlation is involved. Nowadays, the complementary properties of the atomic nucleus, such as its average size, angular momentum, electric and magnetic moments, excitation spectrum, as well as its excitation, decay and reaction probabilities, make up a rich picture in which the correlation between the trends of different observables is the key to understanding nuclear phenomena.

A remarkable example is that of the zirconium $(Z=40)$ isotopic chain which has been studied extensively through different experimental techniques. In particular, the neutron-rich isotopes of zirconium belong to the interesting neutron-rich $A \approx 100$ region of the nuclear chart, which we have already illustrated in Fig. 1.2. A part of the available 
nuclear data for this chain are shown in Fig. 1.6. In the first panel are represented the twoneutron separation energies [Wan12], which at the crossing of the magic neutron number $N=50$ undergo the characteristic two-step drop discussed in the previous section. The magicity of ${ }^{90} \mathrm{Zr}$ is reflected also in the other data of Fig. 1.6. The charge radii of zirconium isotopes, plotted in the second panel as mean-squared differences to ${ }^{90} \mathrm{Zr}$ [Cam97; Cam02; Tha03], show a local minimum at $N=50$. The energy of the first $2^{+}$excited state of the even-even isotopes, represented in the third panel with red symbols [Ram01; Sum11], shows a local maximum for the same neutron number. The electric-quadrupole $(E 2)$ transition probability from the ground state to the first excited $2^{+}$state, represented in the fifth panel by the so-called $B(E 2 \uparrow)$ [Ram01], shows a local minimum. Finally, the magnetic moments of the $N=49$ and $N=51$ zirconium isotopes, represented in the last panel of Fig. 1.6 [Sto11], are close to the Schmidt lines, the idealized limits obtained in the approximation that the odd nucleon (in our case neutron particle or hole) is orbiting around a perfectly inert even-even core [Sch37]. All this experimental information offers a much wider range of evidence upon which the "magicity" of a certain nucleus (essentially an ambiguous concept) can be discussed. Thus, a magic nucleus is not only more bound than the average of its even-even neighbors. It has also smaller average size and it is more difficult to excite, meaning that it requires more excitation energy and yields a lower transition probability for the same final state. For neighbors of magic nuclei it is a good approximation to assume that the dynamics of the nucleons inside the magic core can be neglected and that the properties of the ground state and low-lying excited states are determined only by the valence nucleon, treated as an independent particle or hole. This approximation was proven to hold remarkably well also for many valence nucleons outside a magic core, if one correctly accounts for the correlations between them through a residual interaction. It constitutes the conceptual basis of modern shell-model calculations.

There is still more to observe in Fig. 1.6 than the shell effect at $N=50$. After a relatively straight evolution for the first few isotopes with $N>52$, the two-neutron separation energies undergo another drop between $N=56$ and $N=59$. The charge radii show a slight change of trend at $N=56$, but not as pronounced as the one observed for $N=50$. The energy of the first $2^{+}$excited state has another local maximum at $N=56$ and is unusually high at $N=58$, while the $B(E 2)$ value is correspondingly low at $N=56$. The magnetic moment of the $N=55$ isotope is close to the upper Schmidt limit, suggesting the validity of the independent-particle picture. The combined evidence suggests that $N=56$ corresponds in the zirconium chain to a closed neutron configuration. Indeed, from the early shell-model calculations $N=56$ is already recognized as a closed subshell, meaning that it corresponds to a fully occupied neutron single-particle level, without being separated by a very large energy gap from the first empty state above (as would be the case for magic nucleus). Furthermore, $N=58$ corresponds to the closure of another neutron subshell in the independent-particle picture, supported by the observed 
features of Fig. 1.6.

Nevertheless, only two neutrons more are enough to produce a dramatic change of nuclear structure, as signaled by the sudden increase of the two-neutron separation energy at $N=60$ and $N=61$, briefly also discussed in the previous section. The charge radii jump to a completely new trend at $N=60$, suggesting that protons start occupying quantum states of much larger radial extension and that the single-particle picture collapses into one of strongly mixed configurations. In this new picture, the nucleus is easy to excite with little expense of energy, which leads to the low energy of the first $2^{+}$excited state and large $B(E 2)$ value observed for $N \geq 60$. The pattern of excited states also changes significantly. The ratio between the energies of the first $4^{+}$and the first $2^{+}$excited states (also called the $R_{4 / 2}$ ratio) rises to values close to 3.33, the quantum-mechanical textbook result for a perfectly rigid rotor [Bha92]. This brings into discussion the concept of nuclear deformation, which is also a different way of interpreting the larger mean-squared charge radii of isotopes with $N \geq 60$. The magnetic moments of the first excited $2^{+}$states in the even- $N$ zirconium isotopes are represented with red diamonds in the last panel of Fig. 1.6 [Bha92]. For $N \geq 58$ they approach the $Z / A$ value expected in the simple assumption of a rigid rotation of the proton distribution, while they are closer to 0 between $N=50$ and $N=56$ (see for example [Stu12] for a more detailed analysis). The ground-state magnetic moments of odd- $N$ isotopes depart from the Schmidt limits already at $N=57$. The low-lying $0^{+}$excited state in the even-even zirconium isotopes, represented in the third panel of Fig. 1.6 [Bha92], is called an "intruder" state, because it corresponds to a lowering of the effective gaps in the single-particle energy spectrum as a result of configuration mixing [Hey11]. This state must then have a mixed configuration. At $N=60$, the intruder and the ground $0^{+}$states are almost degenerate in energy, marking a possible inversion point, which is supported by its sudden increase in excitation energy at $N=62$.

Some model concepts, such as configuration mixing or deformation, single-particle or collective, unavoidably emerge in the interpretation of these complementary observables, which cannot be performed in a completely model-independent way. As in the discussion above, these concepts were introduced initially to guide the intuition through the increasing body of nuclear data, and later crystallized as defining concepts of the modern theoretical approaches. A more detailed discussion of the neutron-rich $A \approx 100$ nuclei, including the predictions of nuclear-structure models, will be given in a following chapter. 


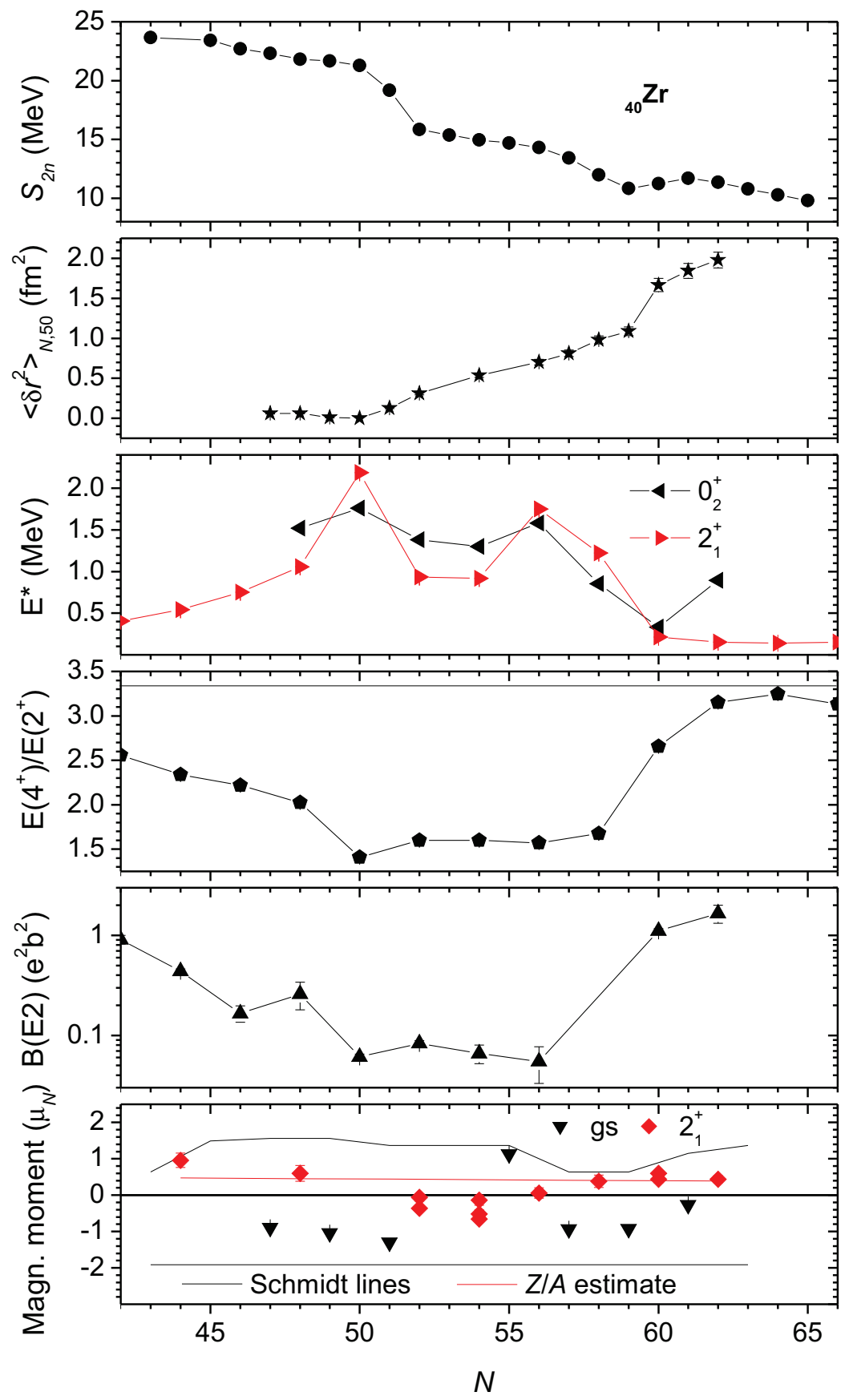

Figure 1.6: Complementary nuclear data for the zirconium isotopic chain. The two-neutron separation energies $S_{2 n}$ are shown in the first panel [Wan12]. The second panel shows the mean-squared charge radii (as displacement from ${ }^{90} \mathrm{Zr}$ ) [Cam97; Cam02; Tha03]. The energies of the first $2^{+}$and $0^{+}$excited states are shown in the third panel, using data from [Ram01; Bha92]. Ratios of the first $4^{+}$and $2^{+}$excited states from [Bha92] are shown in the fourth panel, while $B(E 2 \uparrow)$ values from [Ram01] are shown in the fitfh panel. The final panel shows the ground-state magnetic moments for the odd- $N$ zirconium isotopes from [Sto11] and the magnetic moments of the first $2^{+}$excited states for the even-even isotopes from [Bha92]. For details, see text. 



\section{Chapter 2}

\section{Experimental method and data analysis}

\subsection{Charged-particle traps}

Generally speaking, charged-particle traps are devices which achieve the confinement of charged subatomic, atomic or molecular particles in a finite volume of space, through a combination of electric and magnetic fields (which can be static or dynamic) [Maj05]. Due to their wide range of applications, charged-particle traps have become key components of experimental-physics facilities today [Wer09].

As storage devices, they are indispensable beam-preparation tools, used for the accumulation, bunching, cooling and charge-breeding of primary beams. With these refined techniques, traps act as secondary sources of charged particles with significantly higher density, enhanced phase-space properties, higher charge state, making them more appropriate for low-energy experiments and for post-acceleration [Bla06]. In-trap studies by complementary techniques are also very popular, notable examples being the study of confined, non-neutral plasmas [Maj05], antimatter for fundamental studies [Wid08] and quantum entanglement [Wer09]. Because the motion of charged particles in traps depends on their charge-over-mass ratio $(q / m)$, traps are excellent mass spectrometers, which can be used either to separate a beam in its different $q / m$ constituents or to determine a certain particle's $q / m$ value. Apart from their standalone use for mass spectrometry, their mass resolving power recommends ion traps as excellent beam purifiers [Bla06].

The Penning trap is the combination of a static, quadrupole electrical potential and a static, homogeneous magnetic field. The exceptional advances of the Penning-trap technique since its invention ([Deh68] and references therein) have lead to a series of results of unprecedented precision in fundamental-physics research [Bla10] and turned it into the method of choice for most charged-particle applications mentioned above [Bla06; Wer09]. 
The ISOLTRAP mass spectrometer [Muk08] installed at the ISOLDE facility [Kug00] at CERN was the first experiment to use the advances of Penning traps for the determination of the masses of radioactive nuclides. Since its installation at CERN in the mid eighties, it has contributed significantly to the development of ion purification and measurement techniques and has determined the masses of more than 400 isotopes and isomers [Klu13]. In the remainder of this section, the Penning-trap technique will be presented, focusing on the aspects relevant for its use by the ISOLTRAP experiment.

\section{$2.2 \quad$ Penning trap}

\subsubsection{Dynamics of a trapped ion}

The Penning trap is realized in practice by a set of axially symmetric electrodes, creating the quadrupole potential, placed in a superconducting magnet, producing a homogeneous magnetic field in the region of the Penning-trap center. The symmetry axes of the electric and magnetic field ideally coincide.

The idealized quadrupole electrical potential of a Penning trap is shown in Fig. 2.1(a), where the values are represented by a temperature color map (red indicates higher, blue indicates lower values). To create this potential, one uses a set of three (ideally infinite) electrodes, with the same geometry as the field's equipotential surfaces, which are hyperboloids of revolution. This defines the hyperbolic trap geometry, to which one will be exclusively referring in the following discussion. The black lines represent the intersection with the observation plane of the equipotential surfaces corresponding to the actual electrodes. A three-dimensional image of the three surfaces is represented in Fig. 2.1(b). The characteristic distances of the hyperbolic Penning-trap geometry are marked in Fig. 2.1(a) and are in the case of ISOLTRAP $z_{0}=11.18 \mathrm{~mm}$ and $\rho_{0}=13 \mathrm{~mm}$ [Muk08]. The upper and lower electrodes are called end caps, while the middle electrode is called ring.

A consequence of the Laplace equation is that it is not possible for the electrical potential to be confining in all directions [Bro86]. The gradient of the potential in Fig. 2.1(a) shows that it confines positively-charged particles axially, but not radially. To compensate for this shortcoming, the magnetic field is oriented axially, so that the Lorentz force acting on the charged particle ensures the radial confinement (see Fig. 2.1(b)). The depth of the electrostatic potential well of ISOLTRAP's measurement Penning trap is $\approx 10 \mathrm{eV}$, while the magnetic field is almost $6 \mathrm{~T}$. For achieving this high value of the magnetic field, a superconducting magnet is used. This means that the room-temperature vacuum tube containing the Penning trap is surrounded by a liquid-helium cryostat, which preserves the superconducting state of the magnet. Cryogenic traps and detection systems are also used for high-precision experiments, such as the one reported in [Ulm13].

The detailed derivation of the equations of motion of a particle inside an ideal Penning 


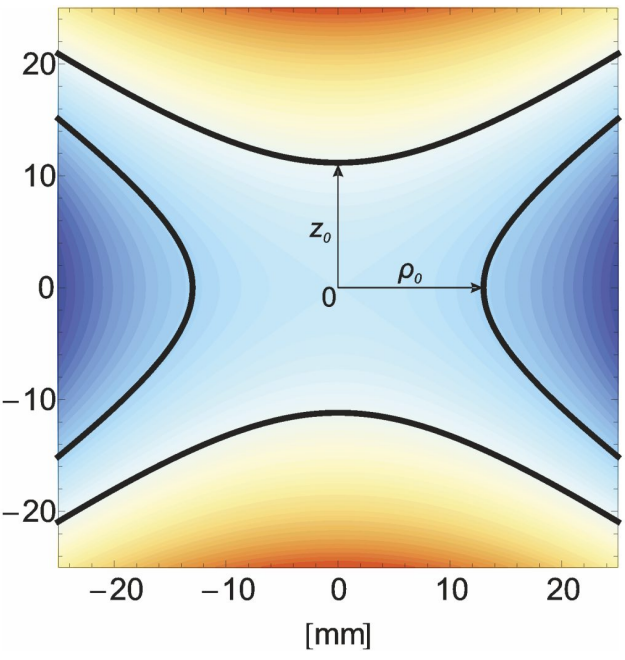

(a)

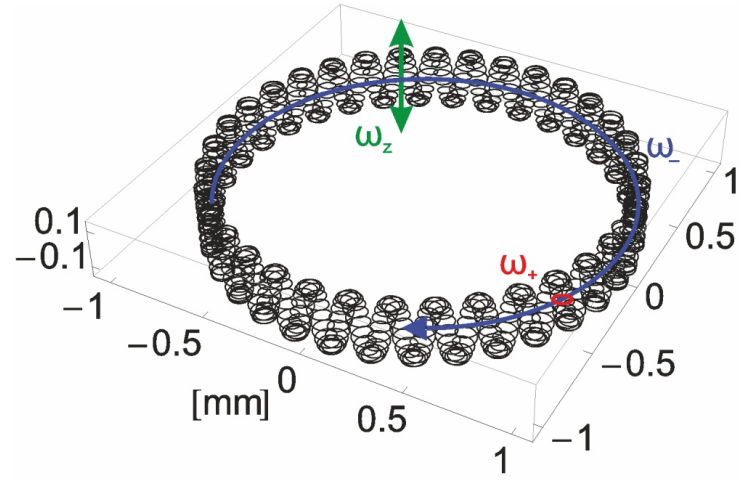

(c)

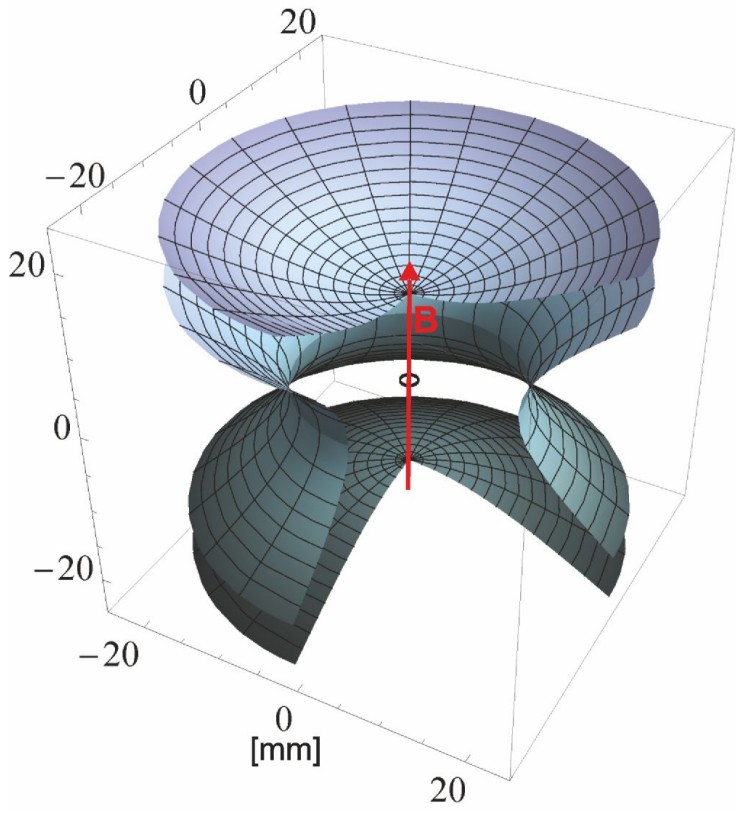

(b)

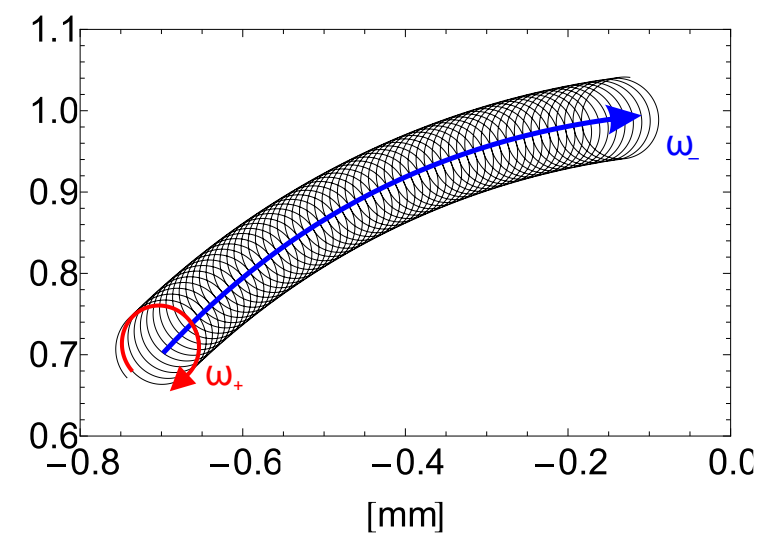

(d)

Figure 2.1: Idealized representations of ISOLTRAP's measurement Penning trap. The quadrupole electrical potential along a certain observation plane is represented as a $2 \mathrm{D}$ temperature map in panel (a). The intersection between the trap electrodes and the observation plane is traced with black lines. A 3D representation of the equipotential surfaces corresponding to the trap electrodes is presented in panel (b), where the orientation of the magnetic field is also marked by a red arrow. The typical trajectory of a singly-charged ion of mass $A \approx 150$ in the Penning trap is shown as a small circle around the trap center in panel (b). A detailed view of this trajectory is presented in panels (c) and (d). In panel (c) the full 3D dynamics is represented, showing the eigenmotions at the axial $\left(\omega_{z}\right)$, magnetron $\left(\omega_{-}\right)$and modified cyclotron $\left(\omega_{+}\right)$frequencies. A closer view of the latter two in the radial plane is presented in panel $(d)$. 
trap is given in Appendix B. Here, only the main results will be summarized. ${ }^{1}$ The axial and the radial motions of the particle are decoupled. The axial motion is a harmonic oscillation around the trap center at the axial frequency $\nu_{z}$, which depends on the electrical potential difference between the end caps and the ring, $V_{0}$ :

$$
\nu_{z}=\frac{1}{2 \pi} \sqrt{\frac{q V_{0}}{m d^{2}}}
$$

where $d^{2}=1 / 2\left(z_{0}^{2}+r_{0}^{2} / 2\right)$ is the characteristic trap dimension. For ISOLTRAP, the axial frequency is $\nu_{z} \approx 35 \mathrm{kHz}$.

The radial motion is the sum of two rotations at different frequencies:

$$
\nu_{ \pm}=\frac{1}{2}\left(\nu_{c} \pm \sqrt{\nu_{c}^{2}-2 \nu_{z}^{2}}\right)
$$

where $\nu_{-}$is called the magnetron frequency and $\nu_{+}$is called the modified cyclotron frequency. The quantity $\nu_{c}=q B /(2 \pi m)$ is the cyclotron frequency of the particle of charge $q$ and mass $m$ in the magnetic field $B$. From Eq. (2.2) one can easily verify the following two very important relations:

$$
\begin{gathered}
\nu_{c}=\nu_{+}+\nu_{-}, \\
\nu_{c}^{2}=\nu_{+}^{2}+\nu_{-}^{2}+\nu_{z}^{2} .
\end{gathered}
$$

In Eq. (2.2) one can factorize the cyclotron frequency and then write the two radial frequencies as a function of the trap parameters:

$$
\begin{aligned}
\nu_{ \pm} & =\frac{1}{2} \nu_{c}\left(1 \pm \sqrt{1-2 \frac{\nu_{z}^{2}}{\nu_{c}^{2}}}\right), \\
& =\frac{q B}{4 \pi m}\left(1 \pm \sqrt{1-2 \frac{m}{q} \frac{V_{0}}{d^{2} B^{2}}}\right) .
\end{aligned}
$$

The cyclotron frequency $\nu_{c}$ of a singly-charged particle of mass $A \approx 100$ in the measurement trap of ISOLTRAP is $\nu_{c} \approx 900 \mathrm{kHz}$. Since this is much larger than the axial frequency $\nu_{z}$, one can approximate the square root in Eq. (2.5) by the first-order Taylor expansion $\left(\sqrt{1 \pm x} \approx 1 \pm \frac{x}{2}\right.$ if $\left.x \ll 1\right)$ :

$$
\begin{aligned}
& \nu_{+} \approx \frac{q B}{2 \pi m}\left[1-\frac{m}{q} \frac{V_{0}}{2 d^{2} B^{2}}\right], \\
& \nu_{-} \approx \frac{V_{0}}{4 \pi d^{2} B} .
\end{aligned}
$$

\footnotetext{
${ }^{1}$ The derivations of Appendix B use the angular frequencies $\omega$, which naturally appear in the equations of motion. Because the measurements always refer to the temporal frequencies $\nu=\omega /(2 \pi)$, the latter will be mostly used throughout this chapter.
} 
Already from the first line of Eq. (2.5), one notices that the magnetron frequency $\nu_{-}$is very small, while the modified cyclotron frequency is only slightly smaller than $\nu_{c}$ (which explains the name). Equation (2.6) shows that in first order the magnetron frequency is mass independent. For ISOLTRAP, the magnetron frequency has the value $\nu_{-} \approx 1 \mathrm{kHz}$.

Figure 2.1(c) shows the three eigenmotions of an ion of mass $A \approx 150$ in ISOLTRAP's measurement Penning trap (idealized). The radii (amplitudes) of the motions are chosen to be similar to actual experimental conditions $\left(\rho_{-}=0.1 \mathrm{~mm}, \rho_{+}=0.05 \mathrm{~mm}, A_{z}=\right.$ $0.15 \mathrm{~mm}$ ). Figure 2.1(b) shows how the trajectory of Fig. 2.1(c) compares to the size of the trap (small circle at the center) and Fig. 2.1(d) illustrates a segment of the projected radial trajectory, which allows better observing the two eigenmotions.

Unlike the representations of Figs. 2.1(a) and 2.1(b), real Penning traps have finitesized electrodes. The end caps have apertures for the injection and ejection of the charged particles and the ring is segmented, to allow driving the ion motion through the application of external variable fields. This causes the field inside the trap to deviate from the pure quadrupole distribution, through higher-order multipole components. To minimize these deviations, correction electrodes are added to the trap design. The success of correcting the field around the trap center with additional electrodes has made it possible to build Penning traps also in cylindrical geometry (one example is the preparation Penning trap installed at ISOLTRAP) [Muk08].

\subsubsection{Driving the ion's motion}

One is interested to have an as low as possible amplitude of the axial motion, to minimize the influence of the trap imperfections on the radial eigenfrequencies. The voltage of the measurement trap's ring electrode and the moment of the ions' capture are optimized to ensure minimal axial oscillation. The radial motions are controlled by applying sinusoidal driving fields on the segments of the ring electrode. The frequencies of the pulses are tuned to produce the resonant excitation of one of the ion's radial eigenmotions, or the resonant conversion of one motion into the other. For excitation, a dipole field is applied (opposite phases on opposite segments, as shown in Fig. 2.2(a)) at one of the radial eigenfrequencies. If the corresponding eigenmotion has no initial amplitude, its amplitude will increase continuously for as long as the excitation is applied, as exemplified in Fig. 2.2(c). For the resonant conversion between the two radial motions, one applies a quadrupole field (opposite phases on segments in quadrature, as shown in Fig. 2.2(b)), tuned to the sum of the two eigenfrequencies (which in the ideal Penning trap is the cyclotron frequency). As a result, one obtains a periodic conversion between the two eigenmotions for as long as the driving field is applied. In Fig. 2.2(d), the conversion between magnetron and modified cyclotron motion is illustrated. Considering the typical frequencies of an ion's eigenmotions in ISOLTRAP's Penning trap, the required driving fields are in the radiofrequency domain and can be produced using standard signal generators. In [Bol90] a detailed description of the different types of excitation is given. 


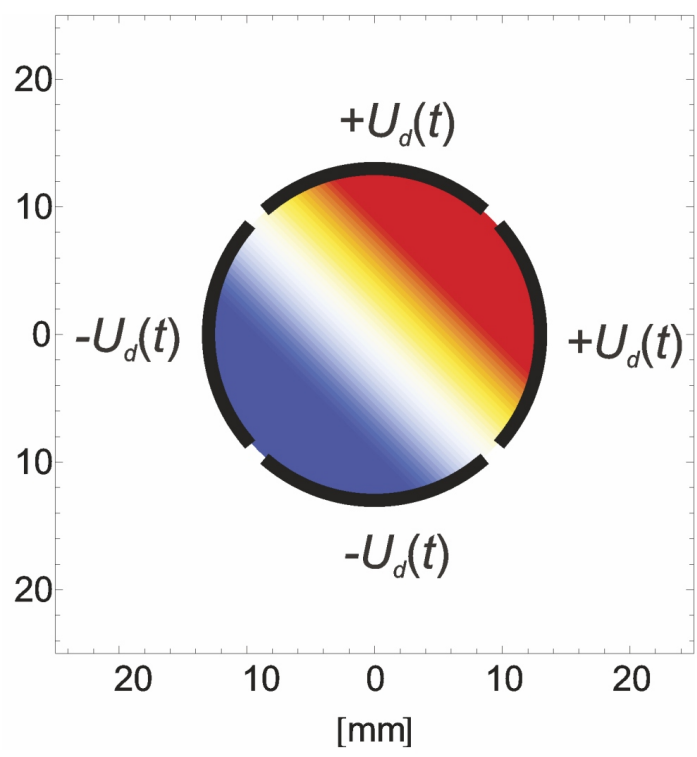

(a)

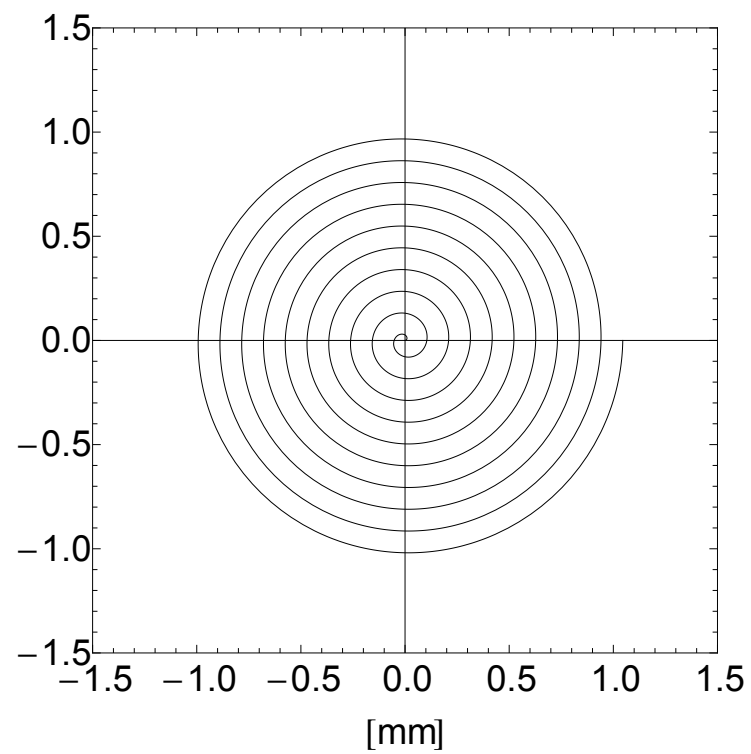

(c)

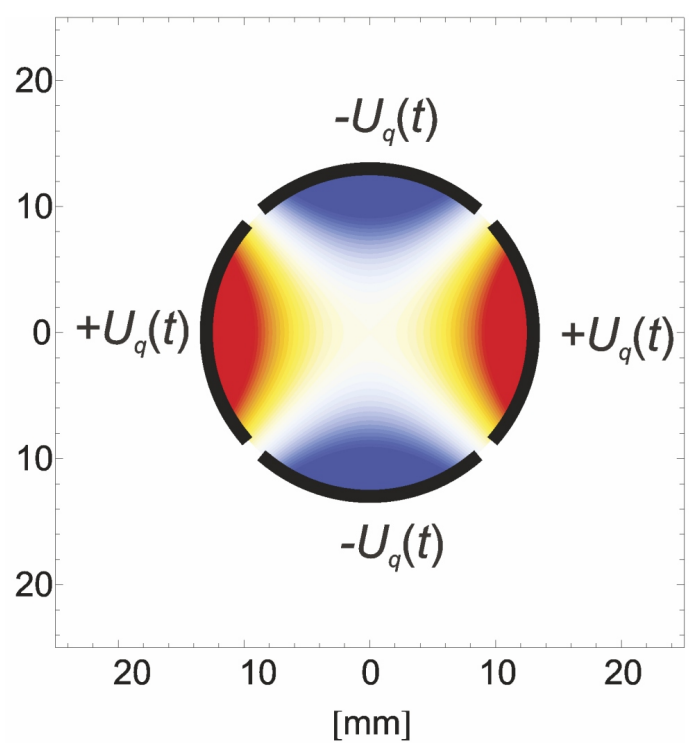

(b)

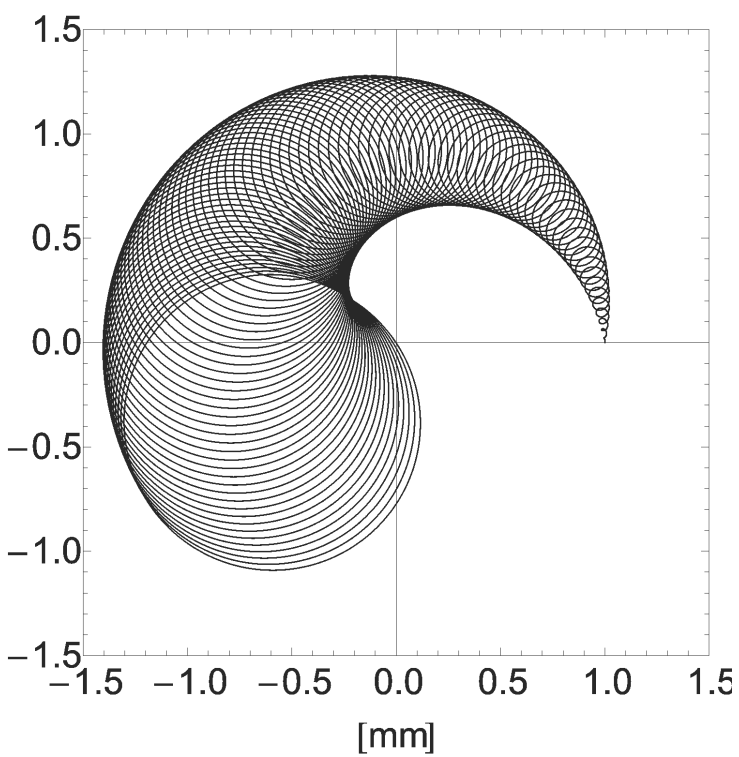

(d)

Figure 2.2: Excitation patterns of the radial eigenmotions of an ion in a Penning trap. The upper panels show the idealized potential distribution for a dipole (a) and quadrupole (b) excitation at zero phase. The lower panels show the resulting ion motion for the two most common excitations applied at ISOLTRAP: (c) dipole excitation at the magnetron frequency and (d) quadrupole excitation at the cyclotron frequency. For panel (d), the ion mass was increased (low cyclotron frequency) as well as the excitation amplitude (fast conversion) in order to enhance the visualization of the process. 
The response of the ion to an external resonant excitation depends on the initial radii and phases of the ion's eigenmotions, as well as the amplitude, phase and duration of the excitation signal. If the eigenmotion has an initial radius, it will be enhanced or reduced by a dipole excitation depending on the phase difference between the eigenmotion and the driving field [Bla03]. The rate at which the radius evolves with time following a quadrupole excitation is proportional to the amplitude of the excitation pulse $U_{e x}$ [Kon95]:

$$
\nu_{\text {conv }}=\frac{q}{m} \frac{U_{e x}}{2 \rho_{0}} \frac{\alpha}{\nu_{+}-\nu_{-}},
$$

where $\alpha$ is a geometric factor. The integral effect of the excitation on the radius of the eigenmotion depends on the length of the excitation pulse. The latter also affects the width of the excitation in frequency domain: the longer the driving pulse is in time, the narrower it is in frequency [Bol90]. A narrower frequency response of the ion to the driving field leads to a higher resolving power of the trap operation.

Controlling the radial motions of an ion in a Penning trap is thus a problem which requires matching the phase of the excitation pulse to the desired effect on the ion's radius, choosing a length of the excitation pulse which ensured a sufficiently high resolving power (narrow frequency response) and adapting the amplitude of the pulse to the desired magnitude of the radius change in the given excitation time.

\subsubsection{Detecting the ion's motion}

There are two main techniques of detecting a charged particle's motion inside a Penning trap and its response to external driving fields.

The first, which has been successfully applied for studying the properties of longlived particles and ions with very high accuracy, uses the charge induced by the moving particle on the electrodes of the Penning trap. This induced charge follows the oscillation of the charged particle and thus its frequency spectrum contains the information on the particle's eigenfrequencies [Deh68]. Amplifying the signal which is picked up from the trap's electrodes and Fourier transforming it offers the possibility of determining a particle's eigenfrequencies in the Penning trap while the particle is still trapped (nondestructive technique). This is called the Fourier-transform ion-cyclotron resonance (FTICR) [Com74], which allows determining a charged particle's free cyclotron frequency by means of Eq. (2.4). With this technique it is possible to perform experiments on a single particle, which can be kept trapped indefinitely [Bro86].

The second technique requires the charged particle to be ejected from the trap and detected (destructive technique, meaning that the particle is no longer available for experiments) [Gra80]. The radial motion of the particle bears a certain magnetic moment:

$$
\mu=\frac{E_{r}}{B}=\frac{\omega_{+}^{2} \rho_{+}^{2}-\omega_{-}^{2} \rho_{-}^{2}}{B} \approx \frac{\omega_{+}^{2} \rho_{+}^{2}}{B}
$$


where $E_{r}$ is the radial kinetic energy and one has used the fact that $\omega_{+} \gg \omega_{-}$.

Along the ions' path from the center of the Penning trap towards the detector, the magnetic fields undergoes a rapid decrease in magnitude (gradient), which causes a coupling to the ejected ions' magnetic moment and creates an accelerating force:

$$
\mathbf{F}=-\boldsymbol{\mu}(\nabla \cdot \mathbf{B})
$$

This force in turn greatly affects the time of flight of the ions from the Penning trap to the detector. Ions without radial motion at the moment of ejection have no magnetic moment and are not accelerated, thus exhibiting the generic time of flight determined by the distance between the trap center and the detector. Ions with radial motion are accelerated and reach the detector faster, exhibiting a lower time of flight (for a more detailed analysis and related formulas discussed in the context of the ISOLTRAP experiment, see for example [Bol90; Kon95]).

This destructive method of detection allows little information to be extracted from the time of flight of a single ion. Instead, one can systematically study a certain property of the ion's motion (e.g. eigenfrequency), or its response to a certain parameter of the driving field (e.g. amplitude), while all other conditions in the trap are kept the same. One usually performs a scan of the frequency or amplitude of the driving field, or of the waiting time before or after the excitation signal is applied [Muk08]. For each step of the scan, an ion (or ion ensemble) is trapped, excited, ejected and detected and the corresponding time of flight to the detector is recorded. The full scan thus requires a minimum number of ions to be produced. By plotting the mean time of flight against the value of the scanned quantity, one obtains a characteristic curve, which contains information about the ion motion inside the trap. Because the electric and magnetic fields between the trap and the detector are well known, the characteristic time-of-flight curves allow also reconstructing part of the ion trajectory inside the Penning trap (such as the amplitudes of the radial motions) [Kon95]. This time-of-flight measurement technique is used at ISOLTRAP. It is preferred to the induced-charge detection method because the latter would require different resonant detection circuits for nuclides in different mass ranges and cryogenic traps and circuits for maximizing the signal-to-noise ratio. Moreover, for most cases of interest the half-life of the studied ions allows only a limited time for analyzing the signal induced on the trap electrodes. Nevertheless, this detection technique is being developed also for experiments on radioactive nuclides [Red13]. The most important application of the time-of-flight detection technique is the determination of the ion's radial eigenfrequencies $\left(\nu_{+}\right.$and $\left.\nu_{-}\right)$and of its free cyclotron frequency $\left(\nu_{c}\right)$. For determining the values of $\nu_{+}$and $\nu_{-}$one applies a dipole driving field on the ring electrode and scans its frequency. For $\nu_{+}$excitation followed by ejection of the ions from the trap is enough. As shown in Fig. 2.3(a) for this type of measurement, the ions' time of flight exhibits a clear variation with the frequency of the driving field. In the figure, all detection events are plotted in a two-dimensional, color-coded histogram. The color code reflects 


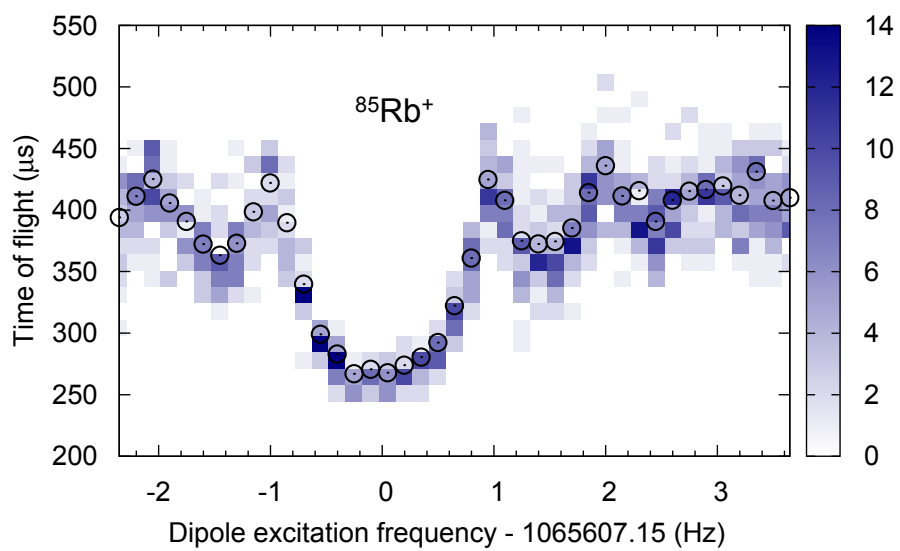

(a)

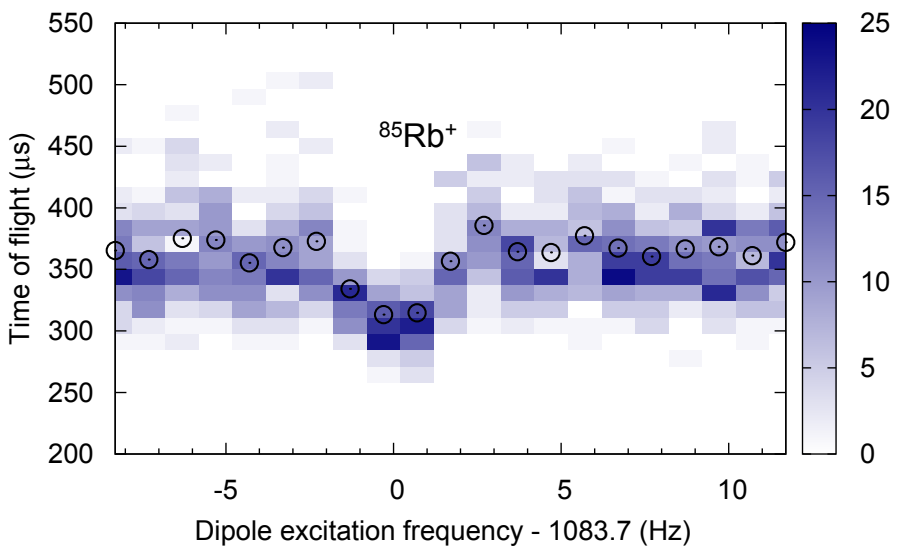

(b)

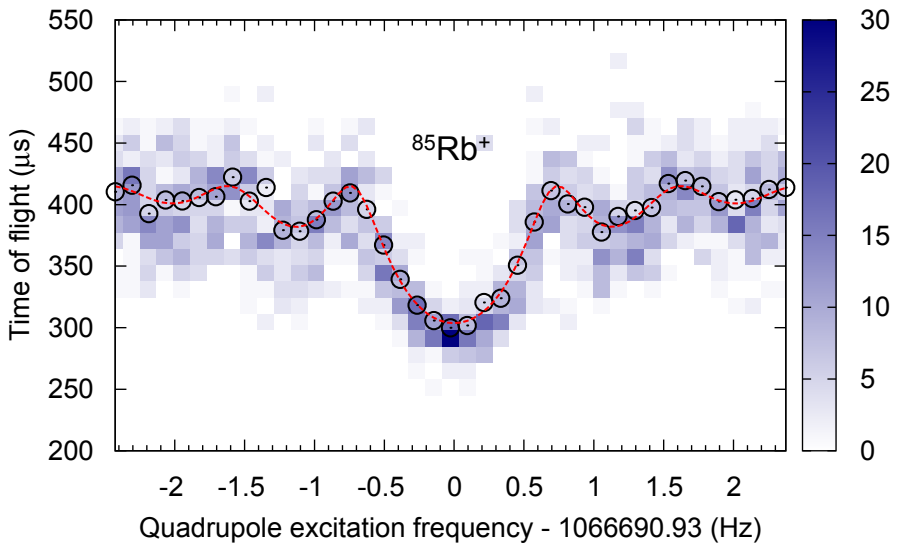

(c)

Figure 2.3: Scans of the frequencies of the main excitations of the ion motion applied at ISOLTRAP in the measurement trap: (a) - dipole excitation of $1 \mathrm{~s}$ length around the modified cyclotron frequency; (b) - dipole excitation of $300 \mathrm{~ms}$ length around the magnetron frequency; (c) - quadrupole excitation of $1.2 \mathrm{~s}$ length around the cyclotron frequency. Each panel is a density plot showing the time of flight of the individual ion-detection events as a function of frequency (darker color represents a higher number of detection events). The mean time of flight of all events corresponding to a certain frequency is marked by black empty circles. For (c), the fit by the theoretical line-shape which allows extracting the cyclotron frequency is also shown by the red dotted line. 
the number of detection events in the corresponding frequency/time-of-flight bin. The average time-of-flight corresponding to each frequency value is represented by the empty circles. In Fig. 2.3(a), the minimum value of the mean time of flight corresponds to the modified cyclotron frequency of the trapped ions (in this case ${ }^{85} \mathrm{Rb}^{+}$). The local minima (side-bands) situated symmetrically with respect to the absolute minimum (resonance frequency) are due to the rectangular envelope of the excitation signal. The amplitude of the frequency response is given by the Fourier transform of the time-domain amplitude [Bol90]. Consequently, the radial energy of the ions, as a function of the excitation frequency, is modulated by the $\sin ^{2} x / x^{2}$ function. One observes that Fig. 2.3(a) exhibits this modulation in reverse, because high radial frequency is converted in the magnetic-field gradient into low time of flight.

In what concerns the determination of $\nu_{-}$, Eq. (2.8) shows that the radial energy of the magnetron motion is too little to produce a significant resonance in time of flight. Following the dipole excitation at a frequency around $\nu_{-}$, one must apply a quadrupole excitation at the true cyclotron frequency $\nu_{c}$ to convert the magnetron motion into cyclotron motion, before the ions are ejected from the trap. The response to the initial excitation of the magnetron motion influences the magnitude of the magnetron radius $\rho_{-}$at the end of the excitation. This becomes the value of $\rho_{+}$following the quadrupole excitation, which through Eq. (2.8) shows that the time-of-flight pattern recorded by the detector reflects the response to the initial excitation of the magnetron motion. This is observed in Fig. 2.3(b), where, as in Fig. 2.3(a), the frequency of the dipole excitation is scanned for ${ }^{85} \mathrm{Rb}^{+}$, but this time around the value of $\nu_{-}$. The figure is similarly constructed as Fig. 2.3(a). A similar pattern is observed, with the global minimum (resonance) giving the magnetron frequency.

The scheme for determining the free cyclotron frequency $\nu_{c}$ is only a slight modification of the one used for the magnetron frequency $\nu_{-}$. In a first step, a dipole excitation at the magnetron frequency is applied, preparing all ions to the same magnetron radius $\rho_{-}$. In a second step, the quadrupole excitation is applied and its frequency is scanned, for a fixed excitation time. The resulting periodic conversion between magnetron and cyclotron motions is resonant when the excitation is applied at the sum of the two frequencies. The amplitude of the quadrupole excitation is matched so that in the resonance case one produces a full conversion of magnetron to cyclotron motion. This means that only on-resonance the final value of $\rho_{+}$is equal to $\rho_{-}$, off-resonance $\rho_{+}$being smaller. By consequence, the final radial energy and time of flight to the detector reflects the frequency response to the quadrupole excitation. This is shown in Fig. 2.3(c), where one notices the same structure with a central minimum and several side-bands, the former corresponding to an excitation at $\nu_{+}+\nu_{-}$and the latter being due to the rectangular envelope of the excitation signal. By computing for each frequency point the mean time-of-flight of all detected ions and fitting the resulting data with the theoretical shape of the resonance curve [Kon95], one can determine the sum of the radial frequencies, which in the ideal 
Penning trap, as shown in Eq. (2.3), is the true cyclotron frequency of the trapped ions. This technique is called the time-of-flight ion-cyclotron resonance (TOF-ICR) [Gra80].

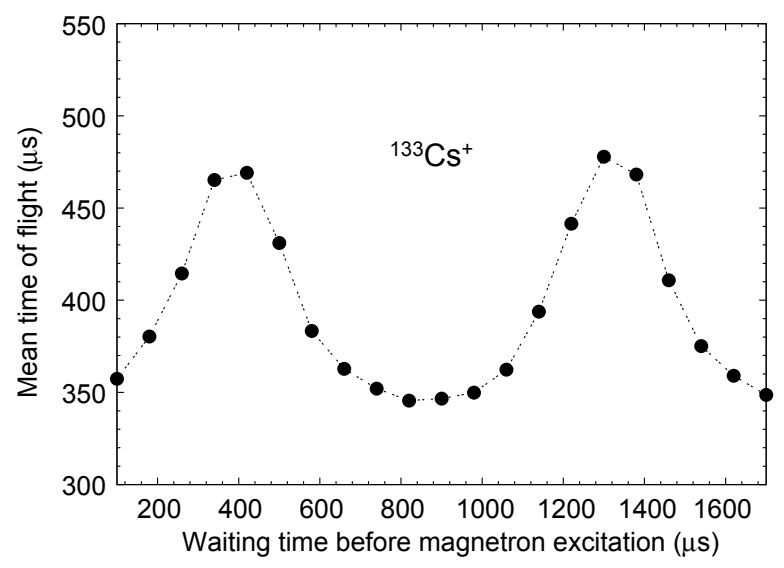

(a)

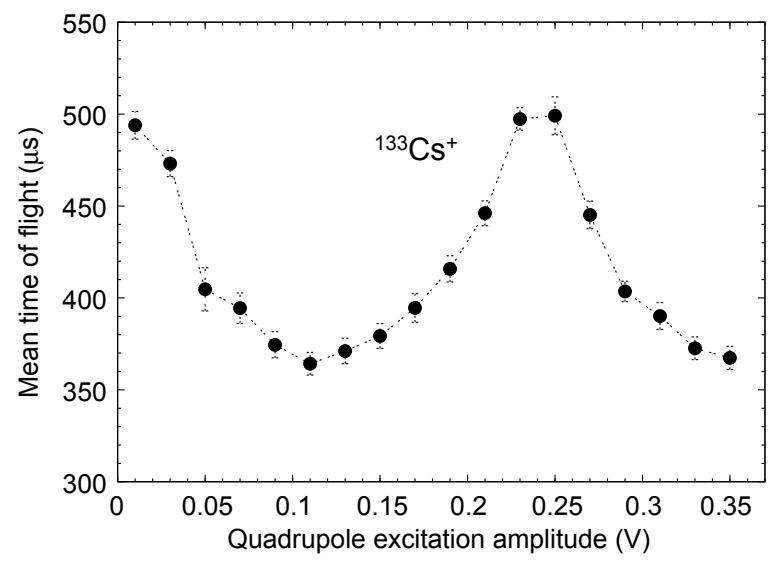

(b)

Figure 2.4: Optimization of the phase and amplitude of some of the excitations of the ions' motion applied at ISOLTRAP in the measurement trap: (a) - scan of the waiting time between the moment of the ions' capture in the trap and the beginning of the magnetron excitation, for fixed amplitudes and resonant excitations; (b) - scan of the amplitude of the quadrupole excitation which converts the magnetron to cyclotron motion, for resonant excitation. Both plots show the mean time of flight of the detected ions for each value of the scanned quantity.

Except for rare cases, due to imperfect injection the ions have an initial magnetron radius $\rho_{-}^{0}$. In order to ensure that the dipole excitation at the magnetron frequency increases from the beginning towards the desired value, the phase difference between the driving field and the ion's magnetron motion must be set to $\pi / 2$ [Bla03]. This is achieved by scanning the waiting time in the Penning trap between the ions' capture and the 
beginning of the dipole excitation. The result of such a scan, performed for ${ }^{133} \mathrm{Cs}^{+}$ions, is shown in Fig. 2.4(a). A phase mismatch leads, for the same amplitude and length of the excitation, to a lower final value of $\rho_{-}$. This becomes the value of $\rho_{+}$after full conversion to modified cyclotron motion, which together with Eq. (2.8) means that a phase mismatch determines a lower radial energy and a larger time of flight. Scanning the waiting time before the magnetron excitation is applied, one observes in Fig. 2.4(a) a modulation of the mean time-of-flight by a periodic function, oscillating at the magnetron frequency. The minima in time of flight correspond to waiting times for which the phase difference between the driving field and the magnetron motion of the ions is $\pi / 2$.

The length of the quadrupole excitation is determined by the required resolving power and precision [Bol90]. The amplitude of the excitation is then optimized to produce exactly one conversion between magnetron and cyclotron motion in this time. To little or too high amplitude will lead to under or over-conversion, both cases meaning that the cyclotron radius at the end of the excitation pulse is lower than the initial magnetron radius. Consequently, the radial energy is lower and the time-of-flight to the detector is larger. As shown in Fig. 2.4(b), scanning the amplitude of the quadrupole excitation and monitoring the mean time of flight of the corresponding ions allows determining the optimal amplitude, which corresponds to the minimum in time of flight situated at lowest voltage. Increasing the amplitude beyond the first minimum eventually leads to a full conversion back and forth between magnetron and cyclotron motions and a local maximum in time of flight. This evolution is periodic, which means that further increasing the amplitude leads to a repetition of the described pattern.

Figs. 2.4(a) and 2.4(b) show that not only the eigenfrequencies of the charged particle inside the Penning trap, but also other features of its motion and excitation can be studied using the time-of-flight detection method. In particular, a study of the axial frequency has also been undertaken at ISOLTRAP [Boh09], in order to verify the invariance theorem Eq. (2.4) [Bro86].

\subsection{From cyclotron frequency to mass: procedure, precision, systematic errors}

The measurement of the cyclotron frequency of a certain ion species in a Penning trap by the TOF-ICR technique allows the determination of its charge-over-mass ratio if one knows the magnetic field at the center of the Penning trap. This can only be done in practice (to enough accuracy) by using the cyclotron-frequency formula in reverse: if one knows the charge-over-mass ratio of an ion species, by measuring its cyclotron frequency one can determine the strength of the magnetic field it was trapped in. These are called reference ions. Labeling the atomic mass of one such species by $m_{r e f}$ and considering that only singly-charged ions are used (which is almost always the case at ISOLTRAP), the 
magnetic field is given by:

$$
\nu_{c, r e f}=\frac{e B}{2 \pi\left(m_{r e f}-m_{e}\right)} \Rightarrow e B=2 \pi \nu_{c, r e f}\left(m_{r e f}-m_{e}\right),
$$

where $m_{e}$ is the electron mass and the electron binding energy was neglected, being much smaller than the achievable precision of the technique. Writing now the cyclotron frequency for the unknown species of atomic mass $m_{x}$, which is also singly charged:

$$
\nu_{c, x}=\frac{e B}{2 \pi\left(m_{x}-m_{e}\right)}=\frac{\nu_{c, r e f}\left(m_{r e f}-m_{e}\right)}{m_{x}-m_{e}}
$$

and introducing the frequency ratio $r_{r e f, x}$ :

$$
r_{r e f, x}=\frac{\nu_{c, r e f}}{\nu_{c, x}}
$$

one can write the mass of the unknown species as:

$$
m_{x}=r_{r e f, x}\left(m_{r e f}-m_{e}\right)+m_{e} .
$$

Equation (2.12) shows that the result of the Penning-trap measurement is not the mass of the studied species (atom, molecule), but the cyclotron frequency ratio of its singly-charged ions with respect to a certain reference ion species. The TOF-ICR technique is thus used to perform relative mass determinations. Referring now only to the isotopes of the different elements, cyclotron-frequency ratios act as links (constraints) between the masses of different nuclides. In the general picture, this adds to links resulting from other mass spectrometry techniques, which also require reference nuclides (Schottky, rigidity, time-of-flight etc.), or from decay and reaction energies [Lun03]. Together, all these links are combined to give a single mass value and a single uncertainty in the atomic mass evaluation [Wan12]. Due to their very high precision, which will be discussed below, cyclotron-frequency ratios from Penning-trap mass spectrometry, if available, usually carry the largest weight of all links attached to a certain nuclide and thus put the strongest constraint on its mass.

From Eq. (2.13) one notices that the statistical uncertainty of the mass of the nuclide of interest is determined by the uncertainty of the cyclotron-frequency measurements (for the ion of interest and for the reference ion) and by the uncertainty with which the reference mass is known. The statistical uncertainty of a cyclotron-frequency measurement is sensitive to three different aspects of the measurement [Bol01], all subject to limitations when measuring radioactive ion species. The first factor is the amount of data acquired (detection events for every frequency of the quadrupole excitation pulse). This is limited for the studied radioactive ions by their production rate at ISOLDE, which is often as low as one 1-10 on average per proton pulse on the target, and by the transport efficiency 
of the ISOLTRAP setup, which is usually around or lower than 1\%. This means that for the duration of an experiment at ISOLDE (typically limited to a few days of continuous measurement), only a limited number of ion-detection events can be acquired. The second factor is the quality of the experimental resonance data. For reasons related to beam purity, in-trap charge production due to radioactive decay (ionization of the rest gas by the decay radiation), preparation of the ion ensembles, optimization of trap excitation pulses and fluctuations of experimental parameters (voltages, amplitudes, digital signals) the experimental data can scatter more or less around the best-fit theoretical line-shape. This experimental noise then translates into the uncertainty of the fitted cyclotron frequency. This uncertainty factor is enhanced when measuring strongly contaminated radioactive ions, because the contaminants have a different frequency response to the quadrupole excitation pulse and thus perturb the average time-of-flight of the ions of interest. Depending on the amount of contamination and on whether it is resolved in the trap (the cyclotron-frequency difference to the ion of interest is larger than the width of the resonance curve), this factor can also lead to a systematic error, which will be discussed later in this section. The third factor is related to the width of the time-of-flight resonance peak around the cyclotron frequency of the measured ion species (giving the resolving power of the excitation). For an excitation time around $1 \mathrm{~s}$ the full width at half maximum (FWHM) of the resonance curve is approximately $1 \mathrm{~Hz}$ and, as discussed before, it scales inversely proportional with the excitation time. A narrower resonance peak allows better constraining the peak center and leads to a more precise determination of the cyclotron frequency. This factor is limited when measuring short-lived isotopes (half-lives lower than $1 \mathrm{~s}$ ), because the count-rate losses by decay become significant, negatively influencing the amount of data which can be acquired. The effect of these factors at ISOLTRAP was translated into the following empirical law for the statistical uncertainty $\delta \nu_{c}$ of a cyclotron-frequency measurement [Bol01]:

$$
\left(\frac{\delta \nu_{c}}{\nu_{c}}\right)_{\text {stat }}=\frac{1}{\nu_{c}} \frac{c}{\sqrt{N} T_{R F}}
$$

where $c$ is a parameter around 0.9 , which slightly depends on the quality of the experimental data [Kel03], $N$ is the number of events and $T_{R F}$ is the length of the quadrupole excitation pulse.

Neither of these three factors is problematic for the measurement of the cyclotron frequency of the reference ion species, because the reference ions used at ISOLTRAP are radioactively stable, abundantly produced and can be easily purified by the available techniques, which will be discussed in the following section. This means that the reference TOF-ICR spectra are high-statistics, pure data sets taken at long excitation time. The reference ions are also used to optimize the transport through the ISOLTRAP setup and the preparation of the ion ensembles for the Penning-trap cycle, meaning that the experimental time-of-flight values follow closely the theoretical line shape of the resonance. 
By consequence, the reference cyclotron-frequency measurements give only a small contribution to the uncertainty of the measured frequency ratio. Because the masses of the reference ions are usually known with very high precision, the statistical uncertainty of the final mass value is dominated by the uncertainty with which the cyclotron frequency of the ion of interest is determined.

Equation (2.14) shows that the relative statistical uncertainty of the TOF-ICR technique, for the same number of detection events and same quadrupole excitation time, varies significantly with the mass of the ion of interest. Relative statistical uncertainties lower than $10^{-7}$ ( $<10 \mathrm{keV}$ for an $A \approx 100$ nuclide) are routinely achieved, but they can be reduced to as low as a few $10^{-8}$ if the production rate and half-life of the nuclide allows [Muk08].

The systematic errors of TOF-ICR measurements have been extensively studied for ISOLTRAP. For most cases they are lower than the statistical uncertainty and represent the uncertainty limit for high-precision experiments (e.g. $Q$-values of interest for neutrino physics and the study of the unitarity of the CKM matrix [Bla10]).

An important source of systematic error is the drift of the trap's magnetic field between the recording of the TOF-ICR for the ion of interest and for the reference ion. The first component of this drift is linear over a measurement's duration and is due to the decay of the current flowing through the superconducting magnet. The second component of the drift is a fluctuation due to changes of the ambient temperature and of the pressure of the helium inside the cryostat housing the magnet [Kel03]. To correct for the linear component of this drift, two reference TOF-ICR measurements are performed, one before and one after the TOF-ICR of the ion of interest. Each reference cyclotron-frequency value is assigned to the time midway between the beginning and the end of the corresponding scan. The value used for computing the frequency ratio of Eq. (2.12) is then the linear interpolation of the cyclotron frequencies determined from the two reference scans at the moment midway between the beginning and the end of the scan corresponding to the ion of interest.

Due to the nonlinear part of the magnetic-field drift between the reference TOF-ICR measurements, this interpolation does not fully correct the systematic error. One way to reduce the error is by implementing a temperature and pressure stabilization system. Such a system is presently not used at ISOLTRAP, but its advantages have been demonstrated [Mar08]. In absence of a stabilization system, studies have been performed to quantify the systematic error due to magnetic-field fluctuations and its magnitude was determined to be [Kel03]:

$$
\left(\frac{\delta \nu_{c}}{\nu_{c}}\right)_{B}=6.35 \times 10^{-11} \Delta \mathrm{T} / \mathrm{min} .
$$

A second source of systematic error is the ion ensemble which is used to perform the measurement. The theory of Penning-trap mass spectrometry presented in this chapter 
and Appendix B is valid for a single trapped charged particle. If more than one particle is present at the same time in the Penning trap, their Coulomb interaction perturbs their motion with respect to the single-particle case. If the ion ensemble is also contaminated, meaning that ions of different masses are present at the same time in the trap, it was shown that the resonance frequencies of all species shift with respect to their free cyclotron frequency. As discussed in [Bol92], a starting point to minimize such effects is to reduce the number of ions allowed in the Penning trap at the same time, so that no more than 3-4 events are detected on average after ejection. Additionally, if the amount of collected data allows it, one also studies, for each data set, how the resonance frequency varies with the average number of detection events (equivalently, of ions in the trap). To this end, one divides the data set in equal subsets corresponding to different average numbers of events. For each subset, one performs a separate fit of the resonance frequency. From the trend of the fitted frequency with average event number, one performs an extrapolation to zero detection events, which is then chosen as the final value of the cyclotron frequency (with the corresponding statistical uncertainty) [Kel03].

A third source of systematic error are the imperfections of the trap electrostatic field, which deviates from the pure quadrupole shape, as well as the misalignment of the trap's electric and magnetic fields [Bol90]. This leads to the so-called "mass-dependent shift," meaning that the cyclotron-frequency ratio deviates from the real value by an amount proportional to the difference in mass between the reference ion and the ion of interest. The magnitude of this error was studied at ISOLTRAP using carbon-cluster ions spanning the entire range of the nuclear chart and was determined to be [Kel03]:

$$
\left(\frac{\delta r_{r e f, x}}{r_{r e f, x}}\right)_{m}=1.6 \times 10^{-10}\left(m-m_{r e f}\right) / u .
$$

The study of [Kel03] showed that, after correcting for the systematic errors resulting from the magnetic-field fluctuations and from the trap-field imperfections, the scattering of the frequency ratios around the expected values leads to a reduced $\chi^{2}$ still greater than one. This is interpreted as a residual systematic uncertainty of the ISOLTRAP mass spectrometer, which is estimated to:

$$
\left(\frac{\delta r_{r e f, x}}{r_{r e f, x}}\right)_{r e s}=8 \times 10^{-9}
$$

A frequency-ratio measurement typically consists of more than one TOF-ICR spectrum of the ion of interest, with corresponding reference spectra. For each of them, the reference cyclotron frequency is interpolated and used, together with the cyclotron frequency of the ion of interest, to calculate the frequency ratio $r_{r e f, x}$ and its statistical uncertainty. For each of the different $r_{r e f, x}$ values, the systematic uncertainty due to the magneticfield fluctuations is calculated, because it depends on the time difference between the associated reference spectra. The uncertainty of each frequency-ratio value $r_{r e f, x}^{i}$ is then 
taken as:

$$
\delta r_{r e f, x}^{i}=\sqrt{\left(\delta r_{r e f, x}^{i}\right)_{s t a t}^{2}+\left(\delta r_{r e f, x}^{i}\right)_{B}^{2}} .
$$

The mean frequency ratio is calculated as:

$$
\bar{r}_{r e f, x}=\frac{\sum_{i}\left[r_{r e f, x}^{i} /\left(\delta r_{r e f, x}^{i}\right)^{2}\right]}{\sum_{i}\left[1 /\left(\delta r_{r e f, x}^{i}\right)^{2}\right]},
$$

while its uncertainty is given by:

$$
\delta \bar{r}_{r e f, x}=\sqrt{\frac{1}{\sum_{i}\left[1 /\left(\delta r_{r e f, x}^{i}\right)^{2}\right]}} .
$$

The value of $\bar{r}_{r e f, x}$ is then corrected by the mass-dependent systematic error and the total uncertainty is calculated as [Kel03]:

$$
\left(\delta \bar{r}_{r e f, x}\right)_{t o t a l}^{2}=\sqrt{\left(\delta \bar{r}_{r e f, x}\right)^{2}+\delta\left(r_{r e f, x}\right)_{m}^{2}+\delta\left(r_{r e f, x}\right)_{r e s}^{2}} .
$$

\subsection{Production and preparation of the ion ensemble}

The nuclides studied with ISOLTRAP are produced by the ISOLDE radioactive ion-beam facility at CERN [Kug00]. Exotic nuclides are produced by proton-induced reactions on a thick target, the material of which depends on the nuclides of interest (ISOL technique [Kos02]). The protons are currently supplied by CERN's proton-synchrotron booster (PSB) in pulses accelerated to $1.4 \mathrm{GeV}$ kinetic energy, providing an average current of up to $2 \mu \mathrm{A}$. For specific experiments, it is interesting to produce the reaction with neutrons, case in which the proton beam is steered towards a tungsten neutron converter placed aside from the target material. In this case, except for unwanted scattered protons, neutrons are the projectiles producing the radioactive nuclides, which changes significantly the production cross sections, in favor of experiments on neutron-rich nuclides (zinc isotopes being a recent highlight [Kos05], [Wol13a]). Studies aimed at optimizing the neutronconverter performance are ongoing [Lui12].

The reaction products are neutralized and their kinetic energy is absorbed in the ISOL target. The target container is heated to high temperature (ca. $2000^{\circ} \mathrm{C}$ ), which leads to the extraction of the nuclides, by the processes of thermal effusion and diffusion, through a transfer line connected to the ISOLDE beam lines (and heated to similar temperatures as the target). The neutral atoms are then ionized by one of three methods. 
One method is the surface ionization on the walls of a heated cavity connected to the transfer line. The efficiency of this method depends on the work function of the cavity for the nuclide of interest. Alkali metals have very low work functions and are thus excellent candidates for surface ionization. This method is however poorly selective (for a given material, the ionization efficiency can only be tuned by changing the temperature of the cavity).

Another method is the resonance-ionization laser ion source (RILIS) [Fed12; Mar13]. Ionization is achieved by overlapping laser beams with the flux of neutral atoms emerging from the target in the cavity of the surface ion source. The resonant character of the ionization is due to the fact that it is achieved by a scheme involving typically two or even three successive atomic transitions. As many laser beams are thus used sequentially to produce the step-wise transition of an electron from its ground level to the continuum. Since the transition energies are element specific, this ionization method is highly selective.

The third method uses a plasma to produce the ionization of the neutral atoms (see [Pen10] for a recent ion-source design). This method is mostly applied to gases and to species which are poorly surface ionized and have no efficient laser-ionization scheme. It is known to produce high currents of different nuclides, but it is a non-selective method.

Of the three ionization techniques, the first two are used to produce exclusively singlycharged ions. The plasma ion source can produce ions in higher charge states. When using RILIS and plasma ionization, the atom extraction cavity still acts as a surface ionizer, which means that all beams are usually contaminated by surface-ionized species. This effect can be reduced by properly choosing the material of the cavity. An outstanding development has been the use of cold quartz lines together with RILIS, to delay some of the surface-ionized contaminants and produce higher-purity beams [Bou08]. Efforts are ongoing for developing the so-called Laser Ion Source Trap (LIST), with the aim of repelling surface-ionized contaminants before performing the laser ionization of the nuclides of interest in a quadrupole ion guide. Preliminary tests at ISOLDE show promising results [Fin13].

Whatever the ionization method, the ion source is floated to a potential of $30-$ $50 \mathrm{kV}$, which accelerates the beam to the corresponding kinetic energy. The singlycharged ions are then transported through one of the two magnetic separators of ISOLDE: the general purpose separator (GPS) or the high-resolution separator (HRS), by which one can typically select from the whole range of reaction products one set of isobars. The HRS can achieve a higher resolving power $(R=m / \Delta m)$ on the order of several thousands (depending strongly on beam preparation), which allows also partially reducing the isobaric contamination of the ion of interest.

The ions are then distributed either to the low-energy or to the high-energy beam line of ISOLDE. The low energy beam line is where ISOLTRAP is situated and uses the beam at its original $30-50 \mathrm{keV}$ kinetic energy. The high-energy beam line starts with a post-acceleration stage. The beam is cooled and bunched in a buffer-gas filled Penning 
trap and bred to a higher charge state by an electron beam ion source (EBIS) [Wol03] and then sent through a system of resonant cavities, which accelerates the beam to energies up to $3 \mathrm{MeV}$ per nucleon [Vou08]. The accelerated beam is then delivered to secondary targets for reaction experiments.

As discussed so far, Penning traps are ideal tools for the determination of the masses of charged particles, however they cannot be used with the ion beams produced at ISOLDE without prior preparation. The ISOLDE beam is a quasi-continuous current of (mostly) singly-charged ions accelerated to 30-50 keV kinetic energy and containing the ions of interest and a significant amount of contamination (usually isobars of the ions of interest, much more abundant in yield). The Penning-trap technique requires this beam to be turned into small bunches of few ions (1-20) at a time, decelerated to effectively zero axial energy and of reduced longitudinal and transverse emittance. Furthermore, the ion bunches should contain ideally only the ion of interest, to avoid the perturbation of their resonance frequency due to Coulomb interaction.

To achieve this, three additional traps constitute ISOLTRAP [Rai97; Her01; Wol12; Wol13b], their role being the preparation of the ion beam for the requirements of the TOF-ICR technique. The schematic of the ISOLTRAP experiment as it stands today is presented in Fig. 2.5 [Kre13]. The ion beam produced at ISOLDE is normally blocked by an electrostatic deflector, which acts as a beam gate. The experimental cycle at ISOLTRAP starts with the opening of the ISOLDE beam gate (deflector voltage set to zero, allowing the beam to pass). The ion beam enters a linear, radio frequency quadrupole ("RFQ cooler and buncher" in Fig. 2.5) [Her01]. The RFQ is sitting on an HV platform and is floated to a potential (in $\mathrm{kV}$ ) slightly lower than the kinetic energy of the ions (in $\mathrm{keV})$. Approaching the RFQ, the ions are decelerated, but still have enough energy to enter the trap. The RFQ is filled with helium buffer gas, which cools the axial and radial emittance of the ions. The four rods making the quadrupole structure are segmented. The different segments are floated to create an axial potential well towards the exit side of the RFQ. In this potential well the ions are accumulated for as long as the beam gate is opened and then kept for 10-20 ms more, to ensure that all ions entering the RFQ have been properly cooled. The bunch of ions formed at the bottom of the axial potential well is then ejected by creating a sharp ramp with the electrodes surrounding the potential minimum.

Before being re-accelerated to their initial kinetic energy, the ions enter a drift tube, which is floated to a potential $\approx 3 \mathrm{kV}$ lower than the RFQ ("1st deceleration cavity" in Fig. 2.5). At the center of the cavity, the ions thus have only $\approx 3 \mathrm{keV}$ kinetic energy, moment in which the cavity voltage is switched to ground potential. Upon exiting the cavity, the ions are no longer accelerated and are further transported at $3 \mathrm{keV}$ kinetic energy. This first step of the experiment thus ensures the bunching, cooling and deceleration of the ISOLDE ion beam.

The $3-\mathrm{keV}$ beam then enters the second trap, the multi-reflection time-of-flight mass 


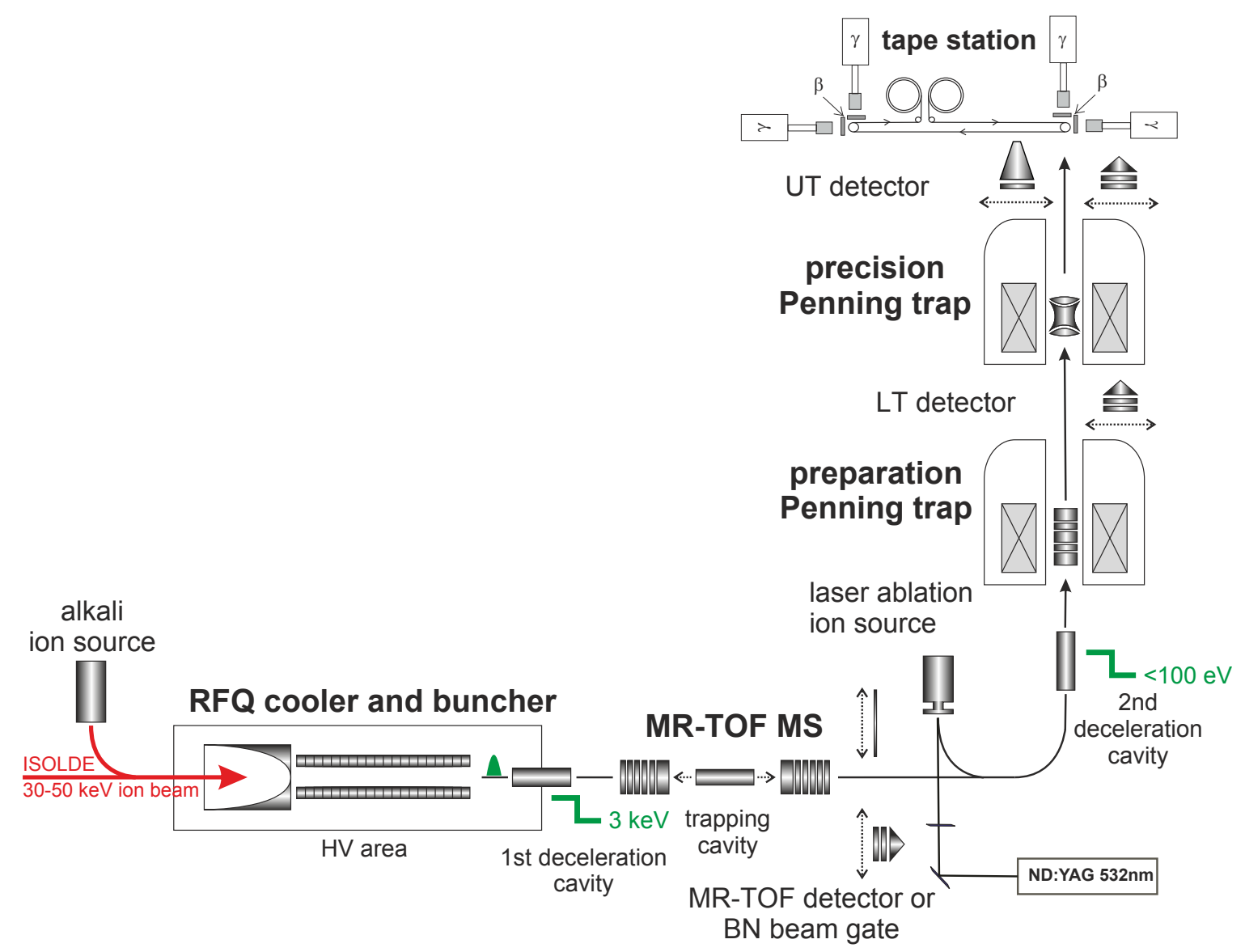

Figure 2.5: Schematic of the ISOLTRAP setup [Kre13] showing the main stages of ion preparation. For details, see text.

separator ("MR-TOF MS" in Fig. 2.5) [Wol12; Wol13b]. The MR-TOF MS is an electrostatic trap, in which the ion bunch undergoes multiple reflections between two sets of mirror electrodes. Initially the ions have enough energy to enter the trap. A drift tube in the center of the MR-TOF MS ("trapping cavity" in Fig. 2.5) is used to capture and to eject the ions, by the same principle which is used with the first deceleration cavity. The ions are decelerated while entering the cavity, which is then switched to ground potential to remove $\approx 1 \mathrm{keV}$ from the ions' kinetic energy, enough for them to start reflecting on the mirror electrodes.

The MR-TOF MS was initially installed in 2010 to act as a beam purifier [Wol12]. Ions of slightly different masses have different velocities inside the device, meaning that after repeated oscillations between the mirror electrodes they start separating in time of flight. With each oscillation, the separation increases, thus increasing the resolving power of the device. As discussed in [Wol13b], there is a competition between the separation of 
the bunches of different ions and the time-of-flight spread of each bunch, both increasing with the number of oscillations. Initially, there is a net gain in resolving power, which reaches values higher than $m /(2 \Delta m) \approx 10^{5}$ for a trapping time of roughly $25 \mathrm{~ms}$. In this time, ions of mass $A \approx 100$ undergo around 1000 oscillations inside the MR-TOF MS (the relationship between number of oscillations and time of flight is mass dependent).

At the exit of the device one can insert a time-of-flight detector, multichannel plate (MCP) or electron multiplier, or a Bradbury-Nielsen beam gate ("BN beam gate" in Fig. 2.5). The detector is used to analyze the arrival times of the ions of different masses. The BN beam gate is an assembly consisting of multiple parallel wires, equally spaced at a distance of $0.5 \mathrm{~mm}$ [Wol12]. Applying opposite voltages on neighboring wires, the gate deflects all ions passing through (closed state). Applying no voltage on the wires, the gate leaves the ions' paths unaffected (open state). By using a fast switch to change the state of the gate from closed to open, one can create a narrow time-of-flight window which only allows the transmission of the ions of interest to the rest of the ISOLTRAP setup. With this technique, a suppression of contaminants of up to $10^{4}$ was shown to be possible [Wol13b]. Figure 2.6(a) shows the suppression of ${ }^{133} \mathrm{Cs}^{+}$on the MCP above the preparation Penning trap (LT detector in Fig. 2.5), with trapping, as a function of the time at which the BN beam gate is opened. For this scan, a $2.5 \mu$ s opening of the beam gate was chosen. Ions are thus transmitted if the gate opening is no earlier than $2.5 \mu \mathrm{s}$ with respect to the arrival time of the ions at the gate. The ion count does not refer to the number of ions in a single bunch. The scan is repeated several times with bunches containing few ions. The suppression of the beam gate can vary with the number of ions in the bunch, especially for large numbers, due to the phase-space distortion brought by the ion-ion Coulomb interaction.

The purified bunch of ions undergoes a $90^{\circ}$ bend and enters the vertical transport section of ISOLTRAP, which contains two Penning traps. The first is the "preparation Penning trap" [Rai97]. It is a cylindrical trap filled with helium buffer gas. Its end caps are set at $100 \mathrm{~V}$, so in order to capture the ions they have to be again decelerated in a drift tube ("2nd deceleration cavity" in Fig. 2.5), by the same method used with the first deceleration cavity. Their kinetic energy is this way reduced from $\approx 3 \mathrm{keV}$ to less than $100 \mathrm{eV}$, which, together with the buffer gas in the preparation Penning trap, ensures that the ions do not have enough energy to exit the trap. To allow the ions' entrance into the trap, the lower end cap is temporarily switched to low voltage.

Before the installation of the MR-TOF MS, the preparation Penning trap was the main device used for the purification of the ion beam, by the mass-selective resonant buffer-gas cooling technique [Sav91]. If the ions' half-life is not a limitation, the trapped ion bunch is initially allowed to cool its axial motion by buffer-gas collisions, for $100-150 \mathrm{~ms}$. The magnetron motion of all ions in the trap is then excited by a short dipole pulse $(\approx 10 \mathrm{~ms})$ and their magnetron radius is increased to a value larger than the ejection aperture of the Penning trap (which until 2012 had a diameter of $3 \mathrm{~mm}$ ). A quadrupole excitation is 
then applied at the true cyclotron frequency of the ion of interest, performing a resonant conversion of magnetron to cyclotron motion. The cyclotron motion is stable, meaning that it is damped by buffer gas collisions, while the magnetron motion, being unstable, is not damped [Bro86]. By consequence, the magnetron radius of the ion of interest is reduced due to the quadrupole excitation pulse, while its cyclotron radius is reduced due to buffer-gas collisions. Following a quadrupole conversion pulse of $\approx 100 \mathrm{~ms}$ length and an additional waiting time of $\approx 50 \mathrm{~ms}$, the resonantly-excited ions are centered in the trap, while the contaminants are still orbiting on a large magnetron radius. At ejection, only the former can pass through the ejection aperture, while the other ions are suppressed.

Figure 2.6(b) shows the effect of scanning the frequency of the quadrupole excitation on the transmission of ions to the MCP situated above the preparation Penning trap (LT detector in Fig. 2.5). Only one ion species is trapped $\left({ }^{133} \mathrm{Cs}^{+}\right)$, which is displaced from the center of the trap and then re-centered resonantly by quadrupole excitation at its true cyclotron frequency. Off-resonance, however, the re-centering doesn't take place and the transmission is zero. Scanning the frequency leads to the characteristic curve of Fig. 2.6(b).

The resolving power of the technique can be as high as $\approx 10^{5}$ for a preparation time on the order a few hundred ms. This time requirement is more than one order of magnitude higher than in the case of the MR-TOF MS, for a similar resolving power. For short-lived isotopes $\left(T_{1 / 2}<100 \mathrm{~ms}\right)$ the resolving power of the trap must be sacrificed in order to reduce the preparation time and minimize losses due to the decay of the ions of interest. For these case, the MR-TOF MS is the method of choice for purification, while the preparation trap is only used for cooling the ion beam. To achieve the ion-beam cooling within the same preparation time, the pressure of the gas inside the trap is increased, typically by an order of magnitude, which allows reducing the trapping time to a few tens of ms.

The ion bunch ejected from the preparation Penning trap is transported to the precision Penning trap, where the TOF-ICR technique is used [Gra80], as described in the previous section. The lower end cap of the precision trap is initially set to a low voltage which allows the entrance of the ions into the trap. The end-cap is raised to its trapping value when the ions are in the center of the trap. Moreover, the ring voltages of the precision and preparation Penning traps are set to the same value. By these precautions, one minimizes the axial amplitude of the ions' motion in the precision trap and ensures that the ions only explore the part of the trap in which the field imperfections are very small. These imperfections can also be minimized by using a set of correction electrodes for the electric field and a room-temperature correction (shim) coil for the magnetic field. The correction procedure used for ISOLTRAP is described in [Bec09].

In case the ions of interest have close-lying contamination which cannot be resolved by the MR-TOF MS or by the purification trap, a final method of purification is available in the measurement Penning trap itself. To this end, after the dipole excitation of the 


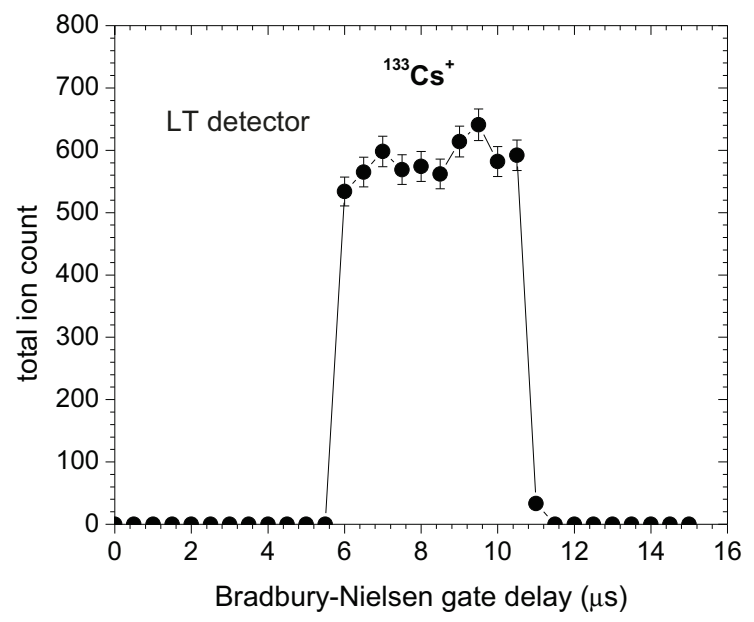

(a)

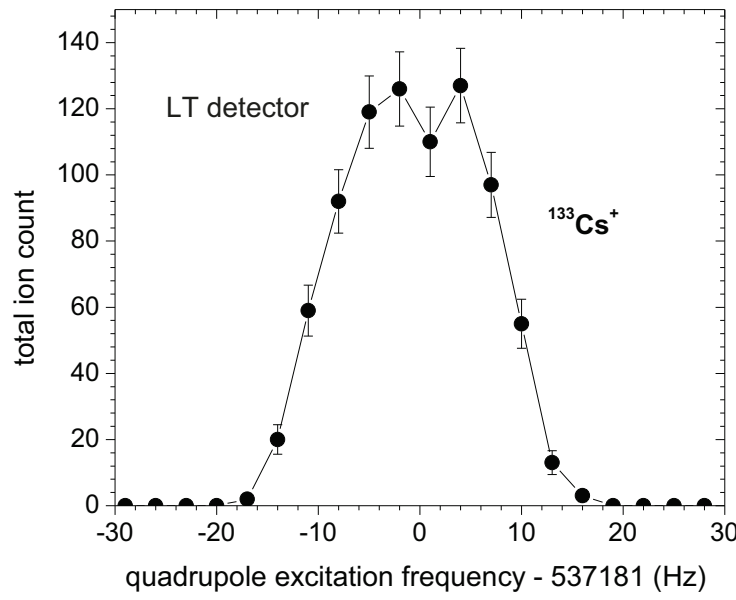

(b)

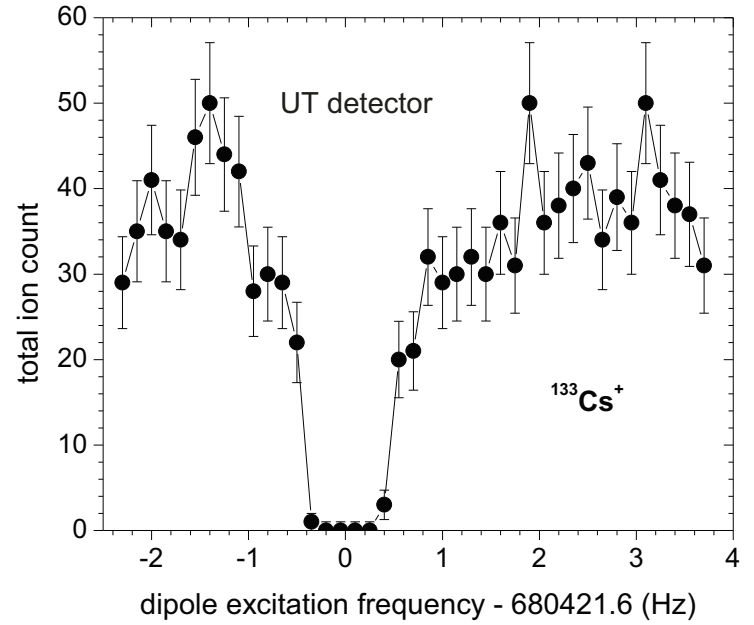

(c)

Figure 2.6: Exemplary scans for the purification techniques used at ISOLTRAP. (a) shows the ion transmission to the LT detector (with trapping) for different opening times of the BN beam gate. The $2.5 \mu \mathrm{s}$ duration of the beam gate opening is reflected in the width of the transmission window. (b) shows the ion transmission to the same MCP for different values of the frequency of the quadrupole excitation in the preparation Penning trap (resonant buffer gas cooling). (c) represents the ion transmission to the UT detector for different values of the frequency of the dipole excitation (dipole cleaning). See Fig. 2.5 for detector position. 
magnetron motion, a dipole excitation of the cyclotron motion can be applied at the modified cyclotron frequency of the contaminating ions. The amplitude of the driving field is large, so that the cyclotron radius of the contaminating ions increases until the ions are lost from the trap [Bec90]. A Gaussian-modulated amplitude is used for the signal, so that the excitation sidebands (see Fig. 2.3(a)) do not excite any remaining ions of interest. For an excitation time on the order of a few s, resolving powers as high as $10^{6}$ can be achieved. A scan of the frequency of the dipole excitation for ${ }^{133} \mathrm{Cs}^{+}$ions in the precision trap is shown in Fig. 2.6(c). The figure shows the transmission to the MCP above the precision Penning trap (UT detector in Fig. 2.5). Off-resonance, the ion transmission is not affected, but when excited resonantly at their modified cyclotron frequency, the ions' radius increases and they are no longer ejected from the trap. This method is however not applicable when the ions of interest are short-lived, because it requires long excitation times.

The high resolving power of ISOLTRAP's Penning-trap purification techniques allows even the separation of nuclear ground and isomeric states [Bol92]. To benefit from this advantage, a decay-spectroscopy setup using a tape station as implantation site was installed above the precision Penning trap ("tape station" in Fig. 2.5). The setup was used used to perform trap-assisted decay spectroscopy. A description of the setup and a report of first results are given in [Kow12; Sta13].

The ion transport through the ISOLTRAP setup and the operation of the four traps are optimized using reference ions, the same ones used for determining the masses of radioactive nuclides from ISOLDE, as discussed in the previous section.

Two types of reference ions are currently available from sources that are part of the ISOLTRAP setup [Kre13]: sodium, potassium, cesium and rubidium ions from a surface ionization source (doped pellet heated to $\approx 1000{ }^{\circ} \mathrm{C}$ ) and carbon-cluster ions (from $\mathrm{C}_{2}$ to $\mathrm{C}_{>20}$ ) produced by a laser-ablation ion source (high-power laser directed at a glassy carbon target). Both sources are represented in Fig. 2.5. The surface ion source has the advantage of producing very stable and easily controllable, low ion currents $(50 \mathrm{pA}-1 \mathrm{nA})$, but the disadvantage of only partially covering the mass range of radioactive nuclides produced at ISOLDE (the heaviest alkali ion produced is ${ }^{133} \mathrm{Cs}^{+}$). This has impact on the achievable transmission efficiency through the setup with radioactive beam. The ion transport is mass dependent due to the existing magnetic fields and, if the optimization is performed with a reference ion very different in mass from the ion of interest, the transmission can end up being significantly worse for the latter. To be more specific, the total efficiency of ISOLTRAP (including detection efficiency) from in front of the ISOLTRAP RFQ to the final detector above the precision Penning trap is typically around $1 \%$ if the ion of interest is close in mass to one of the ions used for optimization, but can drop below $0.1 \%$ when measuring heavy nuclides.

For heavy nuclides, the systematic mass-dependent error also becomes significant if ${ }^{133} \mathrm{Cs}^{+}$is used as reference. For example, in the case of the heavy francium isotope ${ }^{233} \mathrm{Fr}$ 
measured in 2011 with ISOLTRAP, the $\approx 100$ mass-units difference between the ion of interest and the reference ion leads to a relative mass-dependent error of $\approx 2 \times 10^{-8}$, comparable to the achievable statistical uncertainties.

The laser ion source has the advantage of producing carbon clusters over the full mass range of the studied radioactive nuclides, but the disadvantage of more significant countrate fluctuations, due to the strong dependence on the laser power and target condition. Unlike the alkali ion source, which is placed before the RFQ, the laser ion source is placed before the $90^{\circ}$ bender. The carbon clusters are thus not passing through the RFQ and MR-TOF MS and are only cooled an bunched in the preparation Penning trap. They are primarily meant to be reference ions for high-precision measurements, for which the mass-dependent uncertainty needs to be minimized. They can also be used to optimize the ion transport between the two Penning traps, which is mass dependent due to the two magnetic-field gradients. In the past, carbon clusters were used to perform systematic studies and determine the mass-dependent and residual uncertainties of ISOLTRAP [Kel03]. This study needs to be repeated, due to the maintenance of the ISOLTRAP magnets in 2013, which required removing the two Penning traps and much of the vertical optics, discharging the magnets and temporarily moving them out of position. Following their maintenance, they were re-charged and the magnetic-field homogeneity was corrected with the so-called shim coils, followed by a re-alignment of the traps' geometric and magnetic-field axes. All these changes potentially modify the precision trap's systematic errors.

\subsection{Complementary applications of the MR-TOF MS}

The detector placed behind the MR-TOF MS offers a much wider range of applications than the synchronization of the BN-beam-gate opening with the arrival time of the ions of interest. The MR-TOF separation and detection produces a time-of-flight spectrum, which can be calibrated using ions of known mass and converted into a mass spectrum. This information can then be used to identify the composition of the beam produced by ISOLDE and to perform yield studies: the ratio of the number of counts in each timeof-flight peak translates into the relative yield of the corresponding ion species [Wol13b; Kre13].

The time-of-flight calibration of the MR-TOF MS is performed off-line with ${ }^{85} \mathrm{Rb}$ and ${ }^{133} \mathrm{Cs}$ ions. Due to temperature drifts, the time-of-flight values can change by a few $\mu \mathrm{s}$ in the interval of a few days, meaning that the calibration must be repeated shortly before the on-line time-of-flight spectrum is recorded. The drift however refers to the absolute time of flight, the difference in time of flight between the different peaks being much more stable.

An example time-of-flight spectrum obtained with the MR-TOF MS is presented in Fig. 2.7(a). It shows the composition of an $A=152$ ISOLDE beam produced through 
spallation from a tantalum target by $1.4 \mathrm{GeV}$ protons. The dysprosium atoms are ionized using ISOLDE's resonance ionization laser ion source, while the other species are surface ionized in the tantalum extraction cavity. The $A=152$ isobars are selected from the reaction products by the dipole magnet of the GPS.

Laser ionization allows identifying the dysprosium ions by a lasers on/off test (the time-of-flight peak disappearing when the lasers are blocked is the dysprosium peak). With this information one can use the dysprosium peak to correct the time-of-flight drift and identify the other peaks by the relative displacement to it. This procedure is equivalent to performing the calibration of the MR-TOF MS with an on-line reference. This type of calibration is so precise that it can be used not only for identification, but even for determining the mass of the other ion species in the time-of-flight spectrum, if the mass of the on-line reference is well known. Mass determinations with ISOLTRAP's MR-TOF MS were already performed in 2012, showing that relative uncertainties lower than $10^{-6}$ are achievable for a few thousand detection events. The method was applied to determine the masses of the neutron-rich calcium isotopes ${ }^{52-54} \mathrm{Ca}$ [Wie13]. Due to the low yield and short half-life of ${ }^{53,54} \mathrm{Ca}$, combined with the large contamination ratio (predominantly due to ${ }^{53,54} \mathrm{Cr}$ ), the traditional Penning-trap technique could only be used up to ${ }^{52} \mathrm{Ca}$. For this experiment, the MR-TOF MS not only appeared as an alternative mass-spectrometry technique, but as a real option for pushing mass measurements further away from stability than currently possible. The masses of ${ }^{53,54} \mathrm{Ca}$ allowed computing the two-neutron separation energies between $N=32$ and $N=34$ along the calcium isotopic chain and confirmed the prominent shell closure effect at $N=32$ [Wie13].

Coming back to Fig. 2.7(a), using the ${ }^{152} \mathrm{Dy}^{+}$peak as reference, one can determine the expected mean time-of-flight values for the possible isobaric contamination. Considering the spallation cross section and the surface-ionization efficiency, the contamination of the dysprosium beam is expected to consist of neutron-deficient rare-earth nuclides and their molecules. Since many of these species are of valence III, they form diatomic oxide molecules when in the ionic state (valence II). The time-of-flight values computed for comparison thus refer to the neutron-deficient isobars of ${ }^{152} \mathrm{Dy}$ either in the form of single atoms or diatomic oxides. The green lines of Fig. 2.7(a) represent the cases for which a match is found in the MR-TOF spectrum. The assignment is based entirely on the best time-of-flight agreement. The much less abundant species, appearing only as traces in the spectrum, are only tentatively assigned, because of their low yield.

Apart from mass determinations (and implicit identification), the MR-TOF MS can also be used to perform nuclide-specific studies of the ISOLDE ion-beam production parameters. Returning to Fig. 2.7(a). The effect of changing the proton current, target and line temperature, ion source and separator parameters etc. can be studied for each beam component individually. Some illustrative examples are presented in [Kre13].

A critical ISOLDE ion production parameter, the effect of which can be studied using the MR-TOF MS, is the frequency of the lasers producing the resonant ionization of the 


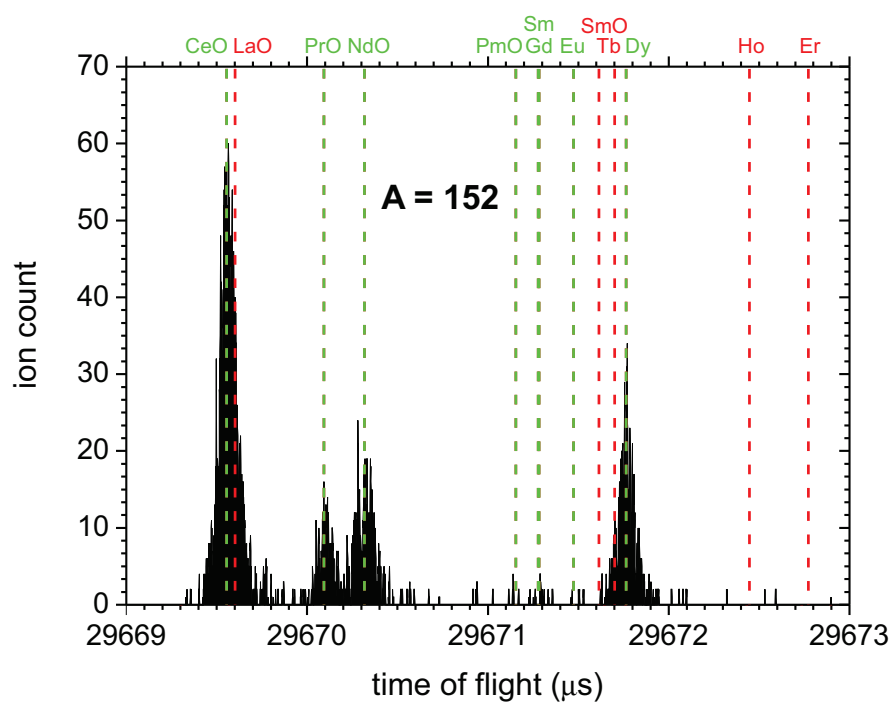

(a)

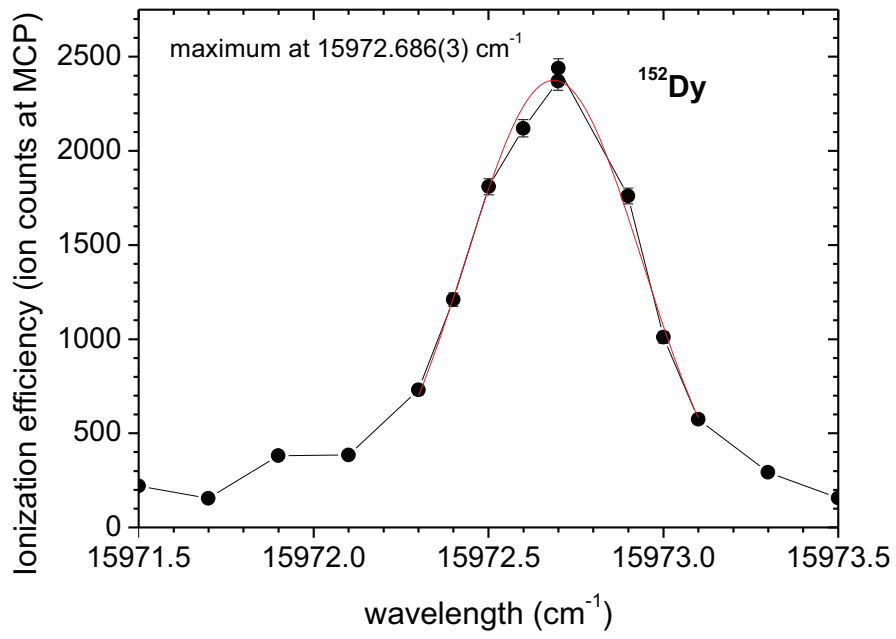

(b)

Figure 2.7: Illustrative examples of applications of the MR-TOF MS as a beam analyzer from spectra recorded on the downstream MCP detector. Panel (a) shows the resolved spectrum of $A=152$ isobars from ISOLDE. Dysprosium is laser ionized and is identified in the spectrum by a lasers on/off test. The vertical dashed lines indicate the expected position, with respect to the center of the dysprosium peak, of the mono-atomic and diatomic-oxide contaminants in the beam (green indicates that the spectrum contains evidence for positive identification based on the best time-of-flight agreement). Panel (b) shows the variation of the ionization efficiency of ${ }^{152}$ Dy with the frequency (expressed in $\mathrm{cm}^{-1}$ ) of the first excitation step of the RILIS scheme. The figure is recorded by gating on the arrival window of the dysprosium ions. The red line represents a Gaussian fit of the ionization efficiency. 
ion of interest. In the case of Fig. 2.7, by gating on the time-of-fight window in which the dysprosium ions arrive at the detector behind the MR-TOF MS, one can analyze how the ionization efficiency of ${ }^{152} \mathrm{Dy}$ varies with the laser frequency, without being impeded by the contaminating isobars. In the example, dysprosium is ionized by a three-step scheme. Figure 2.7(b) shows the variation of the ionization efficiency when scanning the frequency (expressed as wavenumber ${ }^{2}$ ) of the first excitation step. The red line shows a Gaussian fit to the ionization-efficiency curve. The curve does not decrease to zero, because, in the absence of laser ionization, some dysprosium atoms are still surface ionized. The scan is a proof of principle, showing the Gaussian profile of the laser excitation line, Doppler broadened due to the high temperature of the ionization cavity (ca. $2000^{\circ} \mathrm{C}$ ). ${ }^{152} \mathrm{Dy}$ is an even-even isotope, so it has no hyperfine structure (HFS). For sufficiently narrow laser excitation line width, a similar scan would allow accessing the HFS of the atomic levels.

Through the HFS, one can access a whole new range of nuclear properties, complementary to the nuclear binding energy, such as the mean-squared charge radius, spin, magnetic moment and spectroscopic quadrupole moment. The shift of the HFS center of gravity along an isotopic chain (the isotope shift) is also a concern for the ionization efficiency of exotic isotopes, for which the correct excitation frequencies are shifted with respect to those of the stable isotopes. Additionally, if known, the HFS can be in special cases exploited to produce the selective ionization of different long-lived nuclear states (isomers). For example, if the hyperfine splitting of the nuclear ground state is significantly different than the hyperfine splitting of an excited isomer, one can selectively ionize either the ground state or the isomer depending on the chosen frequency of the resonant ionization steps. Since isomers have in many cases a very low excitation energy, making them unresolvable for any purification techniques (including ISOLTRAP's), selective ionization can be the only chance of producing isomerically pure beams for mass spectrometry and other experiments.

Test HFS studies using the MR-TOF MS have indeed shown that it is not only an alternative to other detection methods for resonance-ionization laser spectroscopy, but a valid technique. Traditionally, the ISOLDE in-source spectroscopy studies (with RILIS) use either a Faraday cup to detect the total beam current (for the abundant nuclides close to stability), or the detection of the decay particles (alpha, beta, gamma), for the short-lived nuclides [Mar13]. There is however a class of nuclides (especially the ones intermediate between the two above mentioned extremes), for which the yield is too low to allow Faraday-cup detection, while the contamination and decay mode, or detection rate (half-life, branching ratio, detection efficiency) prevent the use of decay the radiation to estimate the ionization efficiency. For this class of nuclides, the MR-TOF MS is the most efficient method to use, providing contamination-free, laser spectroscopy studies with direct ion detection. In this case, the surface-ionized contamination is not only resolved, but it can also serve as a normalization tool for the hyperfine spectra, allowing correction

\footnotetext{
${ }^{2}$ to convert into units of frequency one needs to multiply by the velocity of light
} 
for any changes in beam production and transport parameters.

Further examples of the complementary applications of ISOLTRAP's MR-TOF MS are presented in [Wol13b; Kre13; Mar13].

\subsection{Experimental results}

During 2011 and 2012 some 15 experiments with radioactive ISOLDE beams have taken place at ISOLTRAP. Some of the experiments were unsuccessful. Others failed to measure the originally intended nuclides, but succeeded in measuring other nuclides or performing complementary studies, including the demonstration of new methods. Approximately a third of the experiments measured nuclear data which were part of the original wish list. Most of the masses resulting from these experiments have been either published already or are close to publication. A list of the resulting articles is presented in Appendix C. In the following, the measurements which were analyzed as part of this thesis' work will be discussed.

\subsubsection{Neutron-rich rubidium isotopes}

The study of neutron-rich rubidium isotopes continued the ISOLTRAP measurements in the neutron-rich $A \approx 100$ region of the nuclear chart. As already discussed in the first chapter for the zirconium isotopes, neutron-rich $A \approx 100$ nuclides undergo an intriguing evolution from the magic neutron number $N=50$ towards the neutron mid-shell at $N \approx 66$, passing from spherical to strongly-deformed shapes through a variety of intermediate configurations. Abundant with complementary experimental evidence, the region has become effectively a textbook case for studying the evolution of nuclear structure resulting from the competing tendencies making up the residual interaction between valence particles. Previous Penning-trap measurements performed with ISOLTRAP have shown however that, in what concerns the mass surface (e.g. two-neutron separation energies), the apparent sub-shell effect at $N=56$ and the sudden onset of deformation at $N=60$ are no longer present in the krypton isotopic chain [Del06; Nai10]. Rubidium isotopes are thus the low- $Z$ border of the region in which all this variety of phenomena takes place and it is interesting to study how their structure evolves further towards the neutron mid-shell.

Neutron-rich rubidium isotopes ${ }^{98-100} \mathrm{Rb}$ were measured by ISOLTRAP as part of the last experiment of 2012. The rubidium isotopes were produced as fission products of the collision between $1.4 \mathrm{GeV}$ protons and a uranium carbide target. They were extracted from the ca. $1800^{\circ} \mathrm{C}$ ISOL target by thermal effusion, surface ionized in a tantalum cavity of ca. $1800-2000^{\circ} \mathrm{C}$ and accelerated to $50 \mathrm{keV}$ kinetic energy. The beams were then transported through the high-resolution separator of ISOLDE and delivered to ISOLTRAP. Due to a favorable combination of production cross section and surface- 
ionization efficiency, the ${ }^{98-100} \mathrm{Rb}^{+}$beams contained much less contamination than ions of interest (strontium isobars being a likely example).

Due to the short half-life of the studied rubidium isotopes, their yield decreased quickly after each proton pulse. The opening of the ISOLDE beam gate was thus synchronized to the proton impact on the target and the ions were each time accumulated in the ISOLTRAP RFQ for a short period of time, ranging from $50 \mathrm{~ms}$ for ${ }^{98} \mathrm{Rb}$ to $200 \mathrm{~ms}$ for ${ }^{100} \mathrm{Rb}$. Due to the high purity of the rubidium beams, the trapping time in the MR-TOF MS was set to only $2.5 \mathrm{~ms}$, yielding a low resolving power. Together with a $110 \mathrm{~ms}$ preparation time in the first Penning trap, this was sufficient to eliminate any potential contamination in the rubidium beam. The ion bunches were then transported to the precision Penning trap, where their cyclotron frequency was measured by the TOF-ICR technique. ${ }^{85} \mathrm{Rb}^{+}$ions were used as references for the measurement. The main parameters of ion production, the trapping times in the MR-TOF MS and in the preparation Penning trap, as well as the number of TOF-ICR measurements and corresponding excitation times are summarized in Table 2.1. A superscript $R$ indicates that the TOF-ICR used a Ramsey-type quadrupole excitation of the ions in the precision Penning trap.

Apart from the traditional TOF-ICR technique with a rectangular envelope of the quadrupole excitation signal, for the studied rubidium isotopes the Ramsey-type excitation was also used [Geo07a; Geo07b]. This type of excitation contains two shorter rectangular pulses, separated by a waiting time. The resonance pattern changes from the one of Fig. 2.3(c), with a large minimum in the middle and small side bands, to a pattern of narrow fringes, shown in Fig. 2.8. The figure is symmetric and not all fringes are as deep as the central one, but because the scan range is centered on the latter it is $a$ priori ambiguous to tell which time-of-flight minimum corresponds to the true cyclotron frequency. For the analysis of the Ramsey-type measurement, a regular TOF-ICR spectrum is still required beforehand in order to fix the center of the Ramsey resonance. Still, because the information in the spectrum is much richer (there are several minima with very sharp slopes) and because the fringes are narrower than the central minimum of the standard resonance, for the same total measurement time, the Ramsey-type TOF-ICR measurement allows better constraining the true cyclotron frequency of the ion of interest by the fit. The result is a lower statistical uncertainty when the Ramsey-type excitation is applied, for the same quadrupole excitation time (including waiting time) and number of detection events. This recommends the Ramsey-type TOF-ICR as an excellent solution for low-yield, short-lived nuclides, for which long excitation times cannot be used in the measurement trap.

Table 2.2 presents the results of the analysis of the ${ }^{98-100} \mathrm{Rb}^{+}$data. For each isotope, the half-life, the frequency ratio with respect to ${ }^{85} \mathrm{Rb}^{+}$and the mass excess of the corresponding atomic species is presented, with the corresponding total uncertainties (statistical and systematic). If already present in the AME, the evaluated mass-excess value is also shown. The main references contributing to the AME values are specified for each 
Table 2.1: Parameters describing the production, preparation and measurement of the rubidium and gold nuclides discussed in this work. The total trapping times for the MR-TOF MS and preparation trap are rounded and displayed in the corresponding columns. The last column presents the number of TOF-ICR measurements and exact excitation time in the precision trap. The superscript $R$ indicates that a Ramsey-type resonance was recorded. For details, see text.

\begin{tabular}{|c|c|c|c|c|c|c|c|c|}
\hline \multicolumn{5}{|c|}{ Production } & \multicolumn{4}{|c|}{ Preparation/Measurement } \\
\hline Date & Target/line & Source & Energy & Sep. & Ion & MR-TOF MS & Prep. trap & Prec. trap \\
\hline \multirow{7}{*}{ Oct 2012} & \multirow{7}{*}{$\mathrm{UC}_{x} / \mathrm{Ta}$} & \multirow{7}{*}{ Ta-surface } & \multirow{7}{*}{$50 \mathrm{keV}$} & \multirow{7}{*}{ HRS } & & & & $2 \times 180 \mathrm{~ms}$ \\
\hline & & & & & ${ }^{98} \mathrm{Rb}^{+}$ & $2.4 \mathrm{~ms}$ & $110 \mathrm{~ms}$ & $2 \times 300 \mathrm{~ms}$ \\
\hline & & & & & & & & $2 \times 400 \mathrm{~ms}$ \\
\hline & & & & & & & & $1 \times 100 \mathrm{~ms}$ \\
\hline & & & & & ${ }^{99} \mathrm{Rb}^{+}$ & $2.4 \mathrm{~ms}$ & $110 \mathrm{~ms}$ & $1 \times 200 \mathrm{~ms}$ \\
\hline & & & & & & & & $2 \times 100 \mathrm{~ms}^{R}$ \\
\hline & & & & & ${ }^{100} \mathrm{Rb}^{+}$ & $2.4 \mathrm{~ms}$ & $110 \mathrm{~ms}$ & $\begin{array}{l}1 \times 50 \mathrm{~ms} \\
1 \times 100 \mathrm{~ms}^{R}\end{array}$ \\
\hline \multirow{4}{*}{ May 2012} & \multirow{4}{*}{$\mathrm{UC}_{x} / \mathrm{Ta}$} & \multirow{4}{*}{ RILIS } & \multirow{4}{*}{$30 \mathrm{keV}$} & \multirow{4}{*}{ GPS } & & & & $1 \times 1.2 \mathrm{~s}$ \\
\hline & & & & & ${ }^{185} \mathrm{Au}^{+}$ & $10 \times 32.7 \mathrm{~ms}$ & $360 \mathrm{~ms}$ & $3 \times 3 \mathrm{~s}$ \\
\hline & & & & & & & & $1 \times 5 \mathrm{~s}$ \\
\hline & & & & & ${ }^{191} \mathrm{Au}^{+}$ & $10 \times 33.2 \mathrm{~ms}$ & $360 \mathrm{~ms}$ & $4 \times 1.2 \mathrm{~s}$ \\
\hline \multirow{6}{*}{ Oct 2012} & \multirow{6}{*}{$\mathrm{UC}_{x} / \mathrm{Ta}$} & \multirow{6}{*}{ RILIS } & \multirow{6}{*}{$50 \mathrm{keV}$} & \multirow{6}{*}{ HRS } & & & & $1 \times 1.2 \mathrm{~s}$ \\
\hline & & & & & ${ }^{180} \mathrm{Au}^{+}$ & $32.2 \mathrm{~ms}$ & $110 \mathrm{~ms}$ & $1 \times 1.8 \mathrm{~s}$ \\
\hline & & & & & & & & $1 \times 2.5 \mathrm{~s}$ \\
\hline & & & & & $188 \mathrm{Au}_{1}^{+}$ & $650 \mathrm{~ms}$ & $110 \mathrm{~ms}$ & $2 \times 1.2 \mathrm{~s}$ \\
\hline & & & & & & $0.0 .9 \mathrm{lns}$ & $110 \mathrm{mls}$ & $1 \times 3 \mathrm{~s}$ \\
\hline & & & & & ${ }^{190} \mathrm{Au}^{+}$ & $33.1 \mathrm{~ms}$ & $110 \mathrm{~ms}$ & $2 \times 1.2 \mathrm{~s}$ \\
\hline
\end{tabular}

isotope.

The mass of ${ }^{98} \mathrm{Rb}$ was already studied with the TITAN Penning-trap mass spectrometer at TRIUMF [Sim12]. An isomeric state is present in ${ }^{98} \mathrm{Rb}$, evaluated in the NUBASE2012 at 600(120) keV excitation energy and $96 \mathrm{~ms}$ half-life, which however was not observed by TITAN. To investigate the presence of this isomeric state in the ISOLDE beam, six standard TOF-ICR measurements of ${ }^{98} \mathrm{Rb}^{+}$were performed, of increasing excitation time (from 180 to $400 \mathrm{~ms}$ ). The measurement of longest excitation time and statistics is presented in Fig. 2.9. The individual ion-detection events are represented by the color map, together with the mean time of flight corresponding to each value of the excitation frequency. The red dashed line marks the fit by the theoretical resonance curve. The detection events at low time of flight $(\approx 170 \mu \mathrm{s})$ correspond to ionized rest gas resulting from the beta decay of the rubidium ions inside the trap. The expected position of the resonance peak of the $600-\mathrm{keV}$ isomer is marked by the vertical dashed line. One observes that the detection events around this excitation frequency do not form 


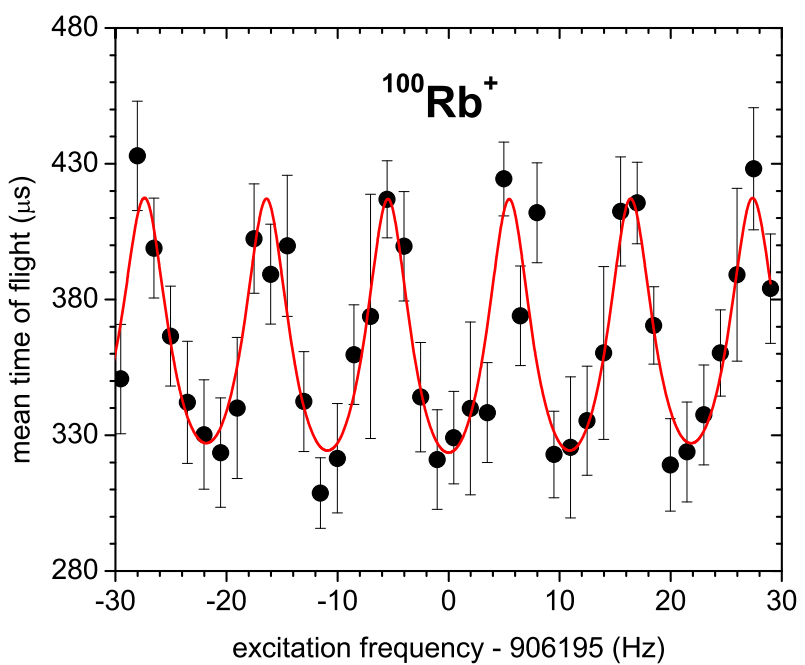

Figure 2.8: TOF-ICR resonance of ${ }^{100} \mathrm{Rb}^{+}$obtained with a Ramsey-type excitation pattern [Geo07b]. For each frequency value, the experimental mean time-of-flight of the ions is represented in full circles. The fitted theoretical resonance curve is represented by the red line [Kre07].

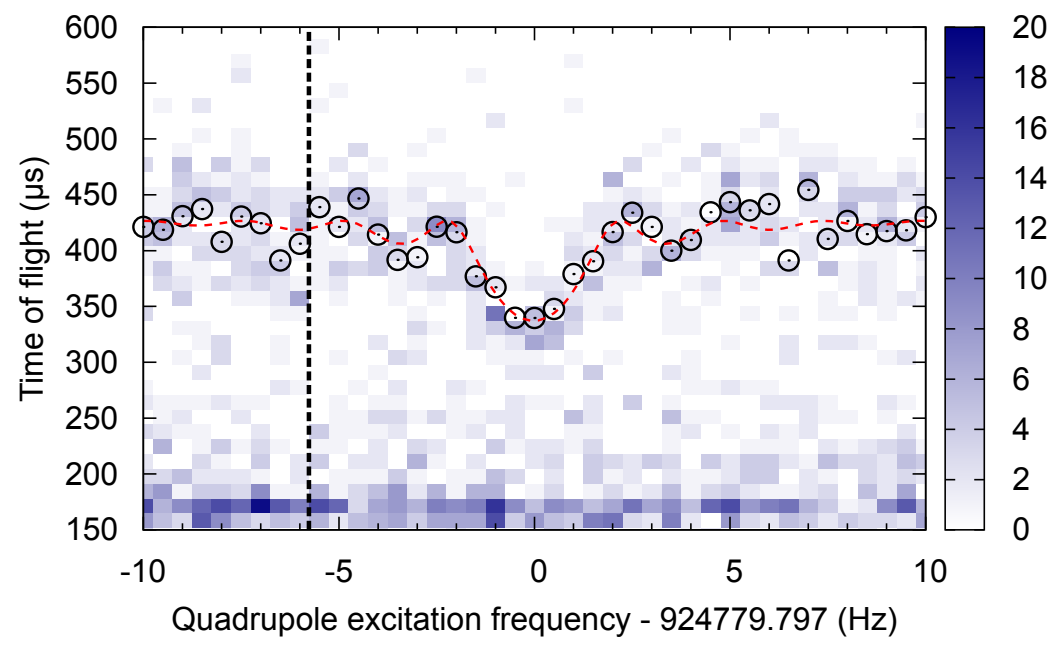

Figure 2.9: TOF-ICR resonance of ${ }^{98} \mathrm{Rb}^{+}$for with a $400 \mathrm{~ms}$ quadrupole excitation time. The experimental time-of-flight of each detection event is represented. The color map reflects the number of counts in each time-of-flight bin. The mean time of flight is also shown for each frequency value (open circles). The fitted theoretical resonance curve is represented by the red dashed line. The vertical dashed line shows the expected position of the resonance minimum corresponding to the isomer in ${ }^{98} \mathrm{Rb}$, according to its excitation energy from [Aud12].

the pattern corresponding to a resonance minimum. Because the isomer and the ground state would be resolved in this frequency scan, the ions of the isomeric state would have to 
appear at a large time of flight above the central resonance minimum. However, nothing of this kind is observed in Fig. 2.9. The conclusion is that, if present in the trapped beam, the ${ }^{98} \mathrm{Rb}$ isomer is much less abundant than the ground state.

The mass of ${ }^{99} \mathrm{Rb}$ is already known in the atomic mass evaluation, constrained by a beta-decay endpoint measurement [Iaf84] and by a mass measurement performed with a double-focusing spectrometer at ISOLDE [Aud86]. The uncertainty of the mass value is however quite high at $110 \mathrm{keV}$. In the ISOLTRAP experiment, two standard TOF-ICR measurements were performed, one with $100 \mathrm{~ms}$ and one with $200 \mathrm{~ms}$ excitation time. To improve the uncertainty, two measurements with Ramsey-type excitation were also performed, with $100 \mathrm{~ms}$ total excitation time (including the waiting time).

The mass of ${ }^{100} \mathrm{Rb}$ was determined for the first time by the ISOLTRAP experiment. Due to the very low yield of the ${ }^{100} \mathrm{Rb}^{+}$beam $(\approx 1000-2000$ ions per proton pulse $)$ and the rapid degradation of the uranium-carbide target condition during the experiment, only two TOF-ICR measurements were performed. The first was a standard TOF-ICR measurement with $50 \mathrm{~ms}$ quadrupole excitation time. The second was a Ramsey-type TOF-ICR measurement with 100 ms quadrupole excitation time. The first measurement, although much less precise, was necessary to determine the center fringe of the Ramseytype resonance. With a half-life evaluated to 48(3) ms in NUBASE2012 [Aud12], ${ }^{100} \mathrm{Rb}$ is one of the shortest-lived nuclides measured at ISOLTRAP by the TOF-ICR technique.

The results of the measurements are summarized in Table 2.2. The mass excess values from the AME2012 are also specified, together with the references which give the major contribution to the evaluated result. One notices that the ISOLTRAP value of the ${ }^{98} \mathrm{Rb}$ mass excess differs by more than two standard deviations from the TITAN value. The determined mass excess of ${ }^{99} \mathrm{Rb}$ agrees with the value of the atomic mass evaluation. The determined mass excess of ${ }^{100} \mathrm{Rb}$ differs significantly from the AME2012 extrapolated value.

Table 2.2: Frequency ratios with respect to ${ }^{85} \mathrm{Rb}$ and mass excess $(M E)$ values of the rubidium isotopes measured in this work [Man13]. The half-lives $\left(T_{1 / 2}\right)$ of the isotopes from NUBASE2012 [Aud12] and the mass excess values from AME2012 [Wan12] are also given ( \# indicates extrapolated values). The last column specifies the references contributing to the AME2012.

\begin{tabular}{llllll} 
Isotope & $\begin{array}{c}T_{1 / 2} \\
(\mathrm{~ms})\end{array}$ & \multicolumn{1}{c}{$r=\nu_{c, \text { ref }} / \nu_{c}$} & $\begin{array}{c}M E_{\text {ISOLTRAP }} \\
(\mathrm{keV})\end{array}$ & $\begin{array}{c}M E_{\text {AME2012 }} \\
(\mathrm{keV})\end{array}$ & References \\
\hline${ }^{98} \mathrm{Rb}$ & $114(5)$ & $1.153453259(51)$ & $-54309.4(4.0)$ & $-54318(3)$ & {$[$ Sim12] } \\
${ }^{99} \mathrm{Rb}$ & $54(4)$ & $1.165270584(57)$ & $-51120.3(4.5)$ & $-51210(110)$ & {$[$ Iaf84; Aud86] } \\
${ }^{100} \mathrm{Rb}$ & $48(3)$ & $1.17710920(25)$ & $-46247(20)$ & $-46550 \#(200 \#)$ &
\end{tabular}

The two-neutron separation energies of the neutron-rich $A \approx 100$ nuclei are represented in Fig. 2.10. The values obtained using exclusively AME2012 masses [Wan12], or using at least one of the rubidium masses from Table 2.2, are plotted with full and empty circles, respectively. One observes that the slight correction of the ${ }^{99} \mathrm{Rb}$ mass by the ISOLTRAP 


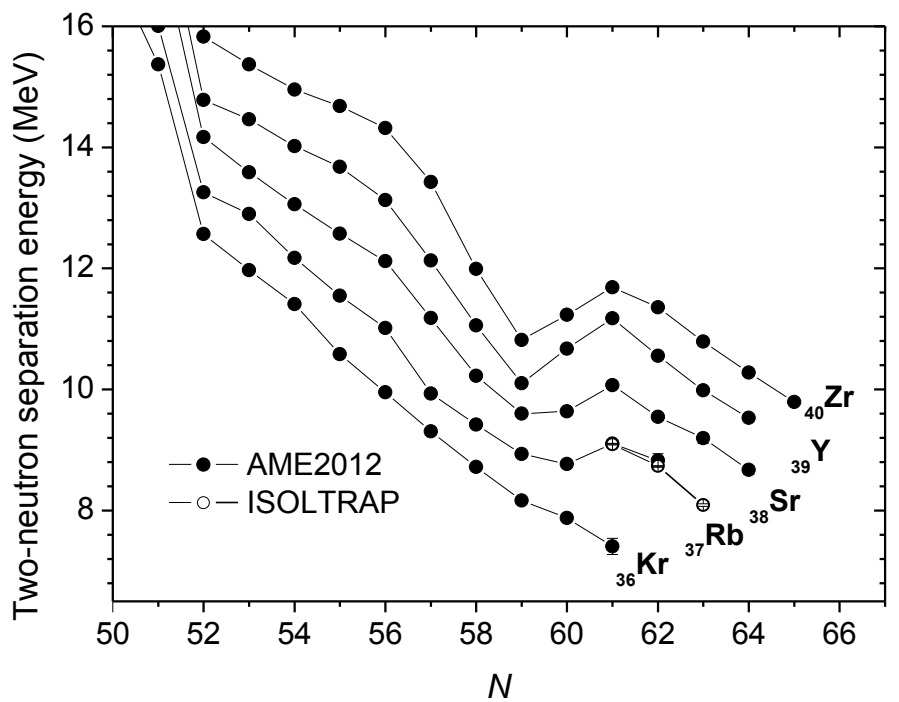

Figure 2.10: Two-neutron separation energies of rubidium isotopes. The full circles represent $S_{2 n}$ values computed using masses from AME2012 [Wan12]. The values obtained using at least one of the new rubidium masses measured with ISOLTRAP [Man13] are shown as empty circles.

value doesn't significantly change $S_{2 n}$ at $N=62$ and that the newly computed $S_{2 n}$ at $N=63$ follows the trend of the previous two isotopes. The region of deformation persist in the rubidium chain at least up to $N=63$. An interpretation of the ground-state masses and charge radii of the neutron-rich $A \approx 100$ nuclei in the framework of self-consistent mean-field theory will be given in Chapter 4 .

\subsubsection{Neutron-deficient gold isotopes}

Neutron-deficient gold isotopes were studied with ISOLTRAP in 2012 during two different experiments. For both experiments, the isotopes were produced as spallation products of the reaction between $1.4-\mathrm{GeV}$ protons and a uranium carbide target. They were ionized in a tantalum cavity by resonance laser ionization (RILIS). The isotopes ${ }^{191} \mathrm{Au}$ and ${ }^{185} \mathrm{Au}$ were produced in the first of the two experiments as a $30-\mathrm{keV}$ beam and purified using the general purpose separator of ISOLDE. The beams of ${ }^{190} \mathrm{Au},{ }^{188} \mathrm{Au}$ and ${ }^{180} \mathrm{Au}$ were produced in the second experiment as a $50-\mathrm{keV}$ beam and were purified by ISOLDE's high resolution separator.

In both experiments, the ISOLDE separators eliminated all but the isobaric contamination of the gold beam. The remaining contaminants were mostly the surface-ionized thallium isotopes, which were however only significantly abundant, with respect to the gold isotopes, on masses $A>185$.

The experimental study had two primary goals. The first goal was to refine the mass 


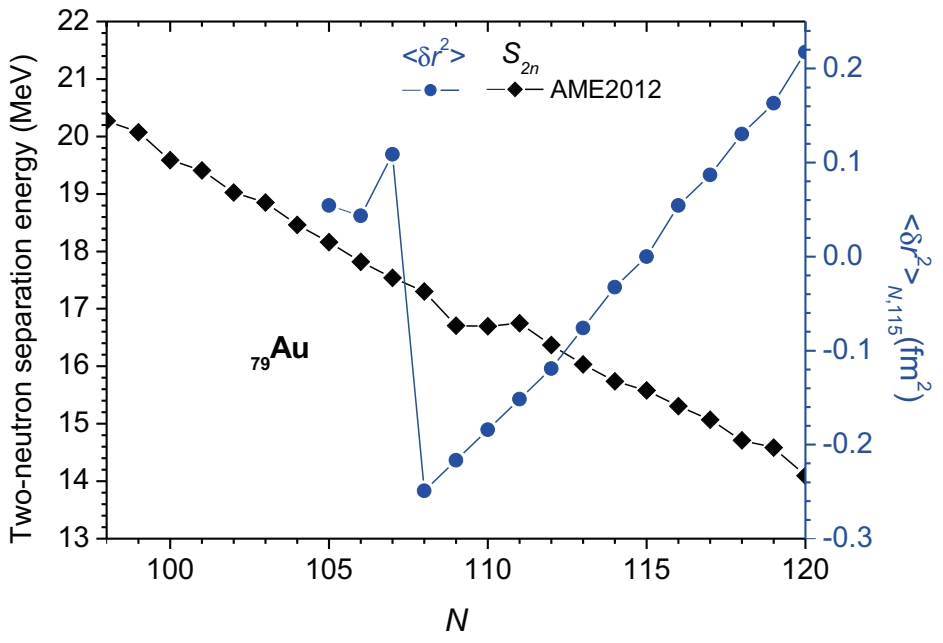

Figure 2.11: Two-neutron separation energies and mean-squared charge radii of the gold isotopes. The $S_{2 n}$ values are computed using masses from AME2012 [Wan12]. The mean-squared charge radii are plotted as differences to the $N=115$ isotope. The evaluated values of [Fri04a] are used. The experimental charge-radii uncertainties are smaller than the used symbols.

surface along the gold isotopic chain. As shown in Fig. 2.11, the mean-squared charge radii of the gold isotopes exhibit a sudden jump between $N=108$ and $N=107$, hinting at an onset of static deformation towards the neutron mid-shell [Wal87; Wal89; Sav90; Bla97]. The two-neutron separation energies of the gold isotopes computed with AME2012 masses [Wan12], also shown in Fig. 2.11, exhibit an interesting behavior between $N=107$ and $N=111$, which seems to reflect a sudden change of nuclear state: a drop between $N=108$ and $N=109$, followed by an enhancement at $N=111$. However, it could also be the result of an underestimation of the binding energy of the $N=109$ gold isotope. Due to its formula which combines the binding energies of nuclei with $N_{0}$ and $N_{0}-2$ neutrons, the $S_{2 n}$ trend would mark this type of error at neutron number $N_{0}$ by a sudden drop between $N_{0}-1$ and $N_{0}$ and by a sudden increase between $N_{0}+1$ and $N_{0}+2$. A re-measurement of the binding energy of the $N=109$ gold isotope could in this context completely smooth out the $S_{2 n}$ trend in the region.

The second goal of the study was the search for isomeric states in the neutron-deficient gold isotopic chain. In the ${ }^{185,188,190} \mathrm{Au}$ isotopes, low-lying isomeric states are proposed in the NUBASE [Aud12] and ENSDF [Bha92] evaluations, at unknown excitation energy. The ordering with respect to the state of determined mass is also ambiguous for these isomers. If present in the ISOLDE beam, the ISOLTRAP purification and measurement techniques could provide a very clean way of determining these isomers' mass and difference in energy with respect to the state already included in the atomic mass evaluation [Wan12].

During both experiments, the gold isotopes were well separated in time of flight from 
their isobaric thallium contamination after 1000 revolutions in the MR-TOF MS. For ${ }^{188} \mathrm{Au}, 2000$ revolutions were used (see Table 2.1 for the corresponding trapping times). Consequently, the Bradbury-Nielsen beam gate was used to suppress the thallium contamination for all studied isotopes. The cycle of the preparation Penning trap was slightly different between the two runs. The first experiment used a long, $\approx 360 \mathrm{~ms}$ cycle, providing a resolving power of a few $10^{4}$. The second experiment was meant to study potentially short-lived states (e.g. isomers in ${ }^{188,190} \mathrm{Au}$ of half-life $\approx 100 \mathrm{~ms}$ ), so the preparation-trap cycle was reduced to one of $\approx 110 \mathrm{~ms}$ length. Nevertheless, the preparation Penning trap still complemented the MR-TOF MS purification with a modest resolving power of $\approx 10^{4}$.

For the long-lived isotopes ${ }^{191,185} \mathrm{Au}$, the so-called "stacking" technique was used. Bunches of MR-TOF purified gold beams were repeatedly injected and accumulated in the preparation Penning trap (for up to 10 times), before being sent to the precision Penning trap for measurement. As discussed in [Ros14], this allowed reducing the effective measurement time required for achieving the same number of detection events, because one decoupled the fast purification part from the slow measurement part of the experimental cycle (dominated by the long quadrupole excitation time in the precision trap). For the isotopes in which short-lived isomeric states were sought, the stacking technique was not used because it would have lead to the decay of the isomeric state in the preparation Penning trap. The main parameters of ion production, the trapping times in the MR-TOF MS and in the preparation Penning trap, as well as the number of TOF-ICR measurements and corresponding excitation times are summarized in Table 2.1. A factor 10 in front of the MR-TOF MS trapping time indicates that the stacking technique was used.

The search for isomers was performed by two different approaches. The first approach aimed at studying the hyperfine structure of the gold isotopes of interest by in-source laser spectroscopy, to exploit the potentially different hyperfine splitting of the isomeric state and achieve its selective ionization. If such a scenario were true, the gain would be twofold: enhancing the isomer's yield and purifying it from the ground state. In the absence of any hyperfine-structure selectivity, the second approach was to transport the ions to the measurement trap and perform high-resolution (long quadrupole-excitation time) TOFICR measurements, for the cases where the isomer's half-life (known or estimated form systematics) allowed it.

For the first approach, the scans were performed in-source (with the RILIS lasers), using the first excitation step of the ionization scheme (the $6 s^{2} S_{1 / 2} \rightarrow 6 p^{2} P_{1 / 2}, 267.7 \mathrm{~nm}$ transition). The ion counts were recorded on the detector behind ISOLTRAP's MR-TOF MS. A complete MR-TOF separation of the background was achieved on all studied gold isotopes, thus the HFS scans were free of contamination. The validity of this approach was confirmed in the case of ${ }^{191} \mathrm{Au}$, for which the hyperfine structures of the known $3 / 2^{+}$ ground-state and $11 / 2^{-}$isomer were recorded. Because the two structures have very different splitting, the two states could be separately ionized and individually measured with ISOLTRAP's Penning-trap system. The results are presented in [Kre13]. 
Figure 2.12 shows a scan of the frequency of the first excitation step of the ${ }^{185} \mathrm{Au}$ ionization scheme. The scanned laser is frequency tripled, which makes the displayed values three times smaller than the actual excitation frequency. Because the electron in the $S$ state has a large overlap with the nucleus, its hyperfine interaction and the resulting splitting of the $S$-level is large. The overlap of the $P$ electron with the nucleus is smaller, leading to a lower splitting of the $P$ atomic level. Considering the transitions between the hyperfine states of the two atomic levels, one expects in the hyperfine spectrum two pairs of closely-spaced resonances (each pair representing transitions from the same $S$ state to the two different $P$ states), separated by a larger frequency interval. Due to the Doppler and power broadening of the laser, the splitting of the $P$ level is not resolved, thus only two peaks appear in the hyperfine spectrum. The red line represents a fit by the theoretical shape of the hyperfine spectrum, for a Gaussian broadening of the line-width. The nuclear spin is assumed to be $I=5 / 2$, which is the known spin of the ${ }^{185} \mathrm{Au}$ ground state. The intensities of the two peaks are left as free parameters, because of possible changes of the laser ionization efficiency during the scan (for example, due to a drift of the laser-beam position in the ionization cavity). The fit allows extracting the center (unperturbed) frequency of the hyperfine structure (CG in Fig. 2.12), from which the isotope shift of the transition (with respect to a reference gold isotope) can be extracted. The systematics of isotope shifts along the gold isotopic chain can then be used to determine the charge radii of the corresponding nuclides. The fit also allows extracting the hyperfine constant $A$ of the $6 s^{2} S_{1 / 2}$ atomic state, which gives access to the magnetic moment of the nucleus. The obtained value of the $A$ factor is in reasonable agreement with the value from the literature [Wal87].

The scan of the hyperfine structure of ${ }^{185} \mathrm{Au}$ extends significantly beyond the position of the two main peaks. This is because the main aim of this scan was not to determine the isotope shift of the ${ }^{185} \mathrm{Au}$ ground state, but to search for additional hyperfine-structure components, which might be related to the ionization of the ${ }^{185} \mathrm{Au}$ isomer. The scan of Fig. 2.12 shows that no such components were identified. Possible explanations are that the isomer is not produced as abundantly, or that its hyperfine structure is not much separated from that of the ground state. Similar scans were performed for ${ }^{190,188,180} \mathrm{Au}$, without revealing any other additional hyperfine structure than the one known from literature. For the short-lived isomers in ${ }^{190,188} \mathrm{Au}$, the slow release from the target is another factor which can contribute to their suppression. However, the performed scans serve as proof of principle of the advantages of using the MR-TOF MS to purify the ion beam, allowing a significant increase of the dynamic range of the scans.

All studied gold isotopes were transported to the measurement Penning trap for determining their cyclotron-frequency ratio with respect to ${ }^{133} \mathrm{Cs}^{+}$. For the ones where the isomer was expected to be long lived (e.g. ${ }^{185} \mathrm{Au}$, possibly ${ }^{180} \mathrm{Au}$ ), TOF-ICR measurements with longer quadrupole excitation time were also performed. None of them revealed a clear signature for an isomeric state being present in the beam. In the case of ${ }^{185} \mathrm{Au}$, 


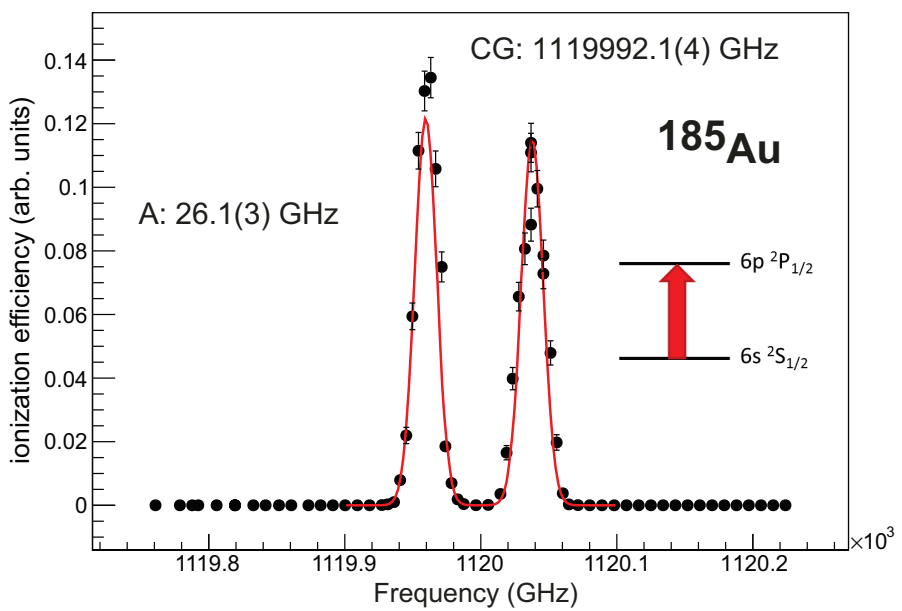

Figure 2.12: Resonance-ionization laser spectroscopy of the $6 s^{2} S_{1 / 2} \rightarrow 6 p^{2} P_{1 / 2}$ transition in ${ }^{185} \mathrm{Au}$, performed using MR-TOF ion separation and time-of-flight-gated detection. The scan is performed in-source, using the first excitation step of the RILIS ionization scheme. Due to the broadening of the laser line-width, only the splitting of the $S$ atomic level is resolved. The red line represents a fit of the spectrum by the theoretical line-shape (for details, see text).

the excitation energy of the isomer is unknown and expected to be very low, which means that, even for a long measurement time, it might still not be resolved from the known state, especially if its yield is significantly lower.

The results of the frequency-ratio measurements for the studied gold isotopes are presented in Table 2.3, together with the resulting mass-excess values. As for the rubidium measurements, the NUBASE2012 half-life [Aud12] and the AME2012 evaluated mass excess [Wan12] of each isotope is specified. The references providing the main links to the masses of the studied isotopes in the AME are also given.

Four TOF-ICR measurements of the ${ }^{191} \mathrm{Au}^{+}$ground state were performed, at $1.2 \mathrm{~s}$ quadrupole excitation time. The very low yield of the resonantly-ionized isomer in ${ }^{191} \mathrm{Au}$ did not allow a precise mass determination, but only an identification of the state based on its excitation energy (see [Kre13]). A Penning-trap mass determination would not have contributed significantly to the mass of the state, due to its very well known excitation energy [Aud12]. The ground-state mass-excess value deduced from the frequency-ratio measurement is in agreement with the AME2012, but reduces the uncertainty by almost an order of magnitude.

Two TOF-ICR measurements of ${ }^{190} \mathrm{Au}^{+}$were performed, with a $1.2 \mathrm{~s}$ excitation time. The determined mass excess shows a significant deviation from the AME2012 $(\approx 50 \mathrm{keV}$, representing three standard deviations of the literature value). A very large deviation of $\approx 90 \mathrm{keV}$ (six standard deviations of the literature value) is also found for ${ }^{188} \mathrm{Au}$, based 
Table 2.3: Frequency ratios with respect to ${ }^{133} \mathrm{Cs}$ and mass excess $(M E)$ values of the gold isotopes measured in this work. The frequency ratio and mass excess of ${ }^{191} \mathrm{Au}$ was already published in [Kre13]. The half-lives $\left(T_{1 / 2}\right)$ of the isotopes from NUBASE2012 [Aud12] and the mass excess values from AME2012 [Wan12] are also given ( \# indicates extrapolated values). The last column specifies the references contributing to the AME2012 value.

\begin{tabular}{llllll} 
Isotope & \multicolumn{1}{c}{$T_{1 / 2}$} & $r=\nu_{c, r e f} / \nu_{c}$ & $\begin{array}{c}M E_{\text {ISOLTRAP }} \\
(\mathrm{keV})\end{array}$ & $\begin{array}{c}M E_{\text {AME2012 }} \\
(\mathrm{keV})\end{array}$ & \multicolumn{1}{c}{ References } \\
\hline${ }^{180} \mathrm{Au}$ & $8.1(3) \mathrm{s}$ & $1.354140704(40)$ & $-25627.3(5.0)$ & $-25594(20)$ & [Kel86; Wau93; Lit05] \\
${ }^{185} \mathrm{Au}$ & $4.25(6) \mathrm{m}$ & $1.391711258(21)$ & $-31858.2(2.6)$ & $-31867(26)$ & [Sii68; Han70; Dau86] \\
& & & & & [Bin91; Lit05] \\
${ }^{188} \mathrm{Au}$ & $8.84(6) \mathrm{m}$ & $1.414279643(22)$ & $-32371.3(2.7)$ & $-32277(15)$ & [Dau84; Lit05] \\
${ }^{190} \mathrm{Au}$ & $42.8(1.0) \mathrm{m}$ & $1.429324264(28)$ & $-32833.5(3.5)$ & $-32883(16)$ & [Joh73; Dio74; Lit05] \\
${ }^{191} \mathrm{Au}$ & $3.18(8) \mathrm{h}$ & $1.43684065(4)$ & $-33797.7(4.9)$ & $-33810(40)$ & [Vie76; Lit05]
\end{tabular}

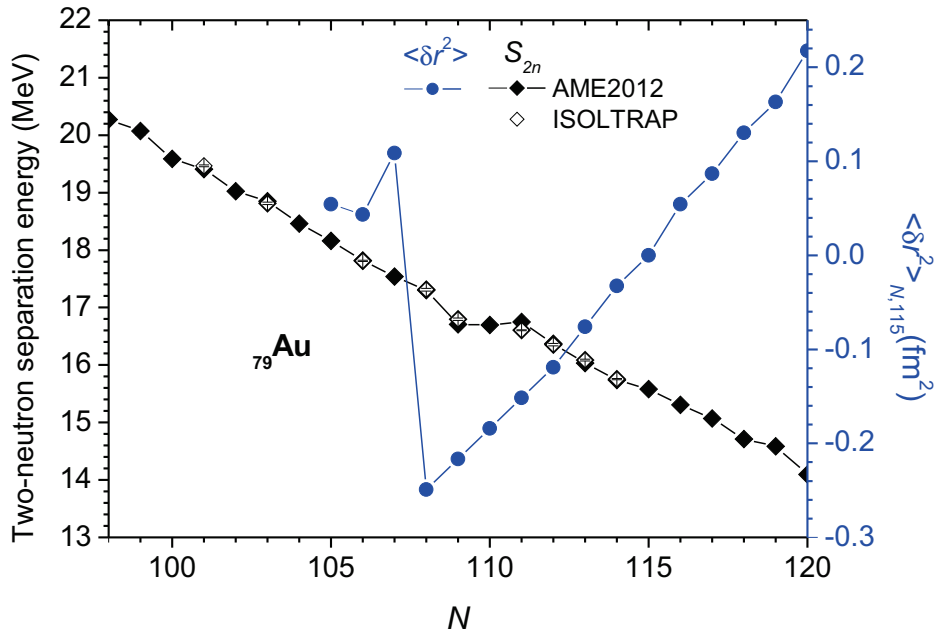

Figure 2.13: Two-neutron separation energies and mean-squared charge radii of the gold isotopes. The full symbols represent $S_{2 n}$ values computed using entirely masses from AME2012 [Wan12]. The open symbols represent $S_{2 n}$ values for which at least one of the contributing masses is a new value analyzed in this work. The mean-squared charge radii are plotted as differences to the $N=115$ isotope. The evaluated values of [Fri04a] are used. The experimental charge-radii uncertainties are smaller than the used symbols.

on three TOF-ICR measurements, two at $1.2 \mathrm{~s}$ and one at $3 \mathrm{~s}$ excitation time. However, no deviation was found for ${ }^{185} \mathrm{Au}$, following five TOF-ICR measurements, one at $1.2 \mathrm{~s}$, three at $3 \mathrm{~s}$ and one at $5 \mathrm{~s}$ excitation time. For ${ }^{180} \mathrm{Au}$ three TOF-ICR measurements were performed at increasing excitation time $(1.2 \mathrm{~s}, 1.8 \mathrm{~s}$ and $2.5 \mathrm{~s})$, leading to a significant improvement of the uncertainty of the mass excess and a correction larger than one standard deviation of the literature value. 
The two-neutron separation energies obtained using the mass-excess values of Table 2.3 are shown in Fig. 2.13. They are compared to the values calculated using exclusively AME2012 data [Wan12] and to mean-squared charge radii from the Fricke evaluation [Fri04a]. One observes that the ISOLTRAP measurements lead to a smoother $S_{2 n}$ trend between $N=108$ and $N=111$. Nevertheless, the $S_{2 n}$ trend is still not perfectly smooth in this region. The drop between $N=108$ and $N=109$ still occurs, as well as what looks like a flattening of the $S_{2 n}$ slope between $N=109$ and $N=111$. This phenomenon will be studied in Chapter 4 in the framework of self-consistent mean-field theory. 


\section{Chapter 3}

\section{Nuclear-theory concepts}

\subsection{Nuclear forces}

Compared to the structure of electrons in atoms, nuclei mark the next dimensional scale in terms of extent, duration and energy quanta of specific processes. The binding energy of electrons occupying the inner-most atomic levels reaches several tens of $\mathrm{keV}$, which also defines their most energetic radiative transitions, situated in the $\mathrm{X}$-ray range. The typical energy of nuclear radiative processes is three orders of magnitude higher, the charged particles and electromagnetic quanta emitted by nuclei having energies of a few $\mathrm{MeV}$. Nuclei are thus systems in full quantum regime and their structure and dynamics is expected to obey the same laws of quantum mechanics as electron systems, but in a new energy domain. Nevertheless, a few fundamental differences have to be considered when passing from electron to nucleon systems, making the description of the latter much more complicated.

First of all, nuclei are composed of two types of particles, protons and neutrons. Electrons are bound by a fixed external potential created by the protons, to which the electron-electron interaction acts as a correction. Nuclei are only (self-)bound by the overall result of the many nucleon-nucleon interaction processes. Furthermore, unlike atomic processes, which are electromagnetic in nature, nuclear processes are governed by the electromagnetic, weak and strong interactions, the latter giving the major contribution to nuclear structure. Unlike electrons in quantum electrodynamics (QED), nucleons are not elementary particles. Their elementary degrees of freedom are the quarks and gluons of quantum chromodynamics (QCD), but nucleons have no overall color charge and these degrees of freedom are not directly involved in the elementary interaction processes which make up the nucleon-nucleon $(N N)$ force. Instead, the strong part of the $N N$ interaction emerges as a residual effect of QCD, modeled in Yukawa theory by the exchange of virtual mesons [Yuk35], the explanation of which is nowadays given in connection to the spontaneous breaking of chiral symmetry [Epe09]. Quantum chromodynamics 
is however non-perturbative at the energy scale of nuclear processes, which are thus very difficult to model from first principles. The standard approach is to approximate the $N N$ interaction through a static potential, using nucleons as elementary degrees of freedom and encoding the hidden QCD physics in effective couplings [Bog10]. The most realistic models, pertaining to the so-called ab initio methods [Sto94; Wir95; Mac01] restrict the possible couplings of the $N N$ potential by imposing a very general set of symmetries of the strong force. Its spatial dependence is modeled phenomenologically or, for the longand intermediate-range parts, by using Yukawa-type, meson-exchange potentials [Rin00; Epe09; Bog10]. The free parameters are then constrained to describe the experimental $N N$ scattering data.

Even so, these realistic (or "bare") $N N$ potentials are very difficult to use in practical calculations due to their strong repulsive ("hard-core") and tensor components at short distance. For nuclear-matter and nuclear-structure calculations, the Brückner G-matrix method has been used for a long time to construct an in-medium effective interaction from the bare one [Rin00]. More recently, renormalization-group techniques have been exploited to build low-momentum interactions, significantly improving their appropriateness for numerical calculations [Bog10]. In this context one discusses the emergence of manybody terms (such as three-body and higher order) in the $N N$ potential, the strength of which strongly depends on the resolution associated to the momentum scale for which the potential is defined. Three-body forces turn out to be essential for obtaining a proper description of light nuclei even at the level of the "hard-core" potentials [Pie01], their role being related to the inner structure of the interacting nucleons. With the advancements in effective field theory (EFT), chiral-EFT has emerged as a promising approach to building effective $N N$ potentials consistent with the symmetries of QCD, in which the two-, three, ... n-body terms of the interaction emerge consistently within the same theoretical framework [Epe09; Bog10].

\subsection{Many-body calculations}

In nuclear-structure calculations one needs to solve the Schrödinger equation

$$
\hat{H}|\psi\rangle=E|\psi\rangle,
$$

for the many-body Hamiltonian $\hat{H}$ :

$$
\hat{H}=\sum_{i=1}^{A} \hat{t}(i)+\sum_{i<j}^{A} \hat{v}(i, j),
$$

which contains, apart from the kinetic-energy terms $\hat{t}(i)$, the $N N$ potential $\hat{v}(i, j)$, depending on the spatial, spin and isospin coordinates of the particles $i$ and $j$ [Rin00]. For simplicity, Eq. (3.2) only contains a two-body potential. 
At this point, one adds to the complications of fundamental nature mentioned in the previous subsection the complexity of the nuclear many-body problem itself, which has made it impossible so far to solve the Schrödinger equation for more than just the lightest of atomic nuclei, and this already at great computational cost [Pie01]. The description of nuclear structure is thus not only plagued by uncertainties in defining the effective nuclear potential, but also by the many approximations one makes in order to achieve a numerical result in reasonable computational time. In what nuclear binding energies are concerned, changes are small and rather smooth with the change of proton and neutron number. Considering also the great numerical simplification they bring, it is not surprising that macroscopic models with shell corrections have been so popular approaches until very recently (worth mentioning is the finite-range droplet model, one the most developed and successful versions of these models [Mol95]). Nevertheless, to describe other quantities than the nuclear binding energy, size and shape, and to be able to constrain the properties of the $N N$ potential on nuclear data, pure microscopic approaches are required.

The two main microscopic models which have been so far used to calculate the properties of a wide range of nuclei are the Hartree-Fock-Bogoliubov approach [Ben03] and the nuclear shell model [Cau05]. The former will be introduced in more detail in the following section, the latter will be briefly described here. Both models start from the idea of extending the independent particle approximation, successfully applied in atomic physics, to the nuclear case. This is equivalent to saying that the interaction of a certain nucleon with all the other protons and neutrons can be approximated at least in first order by a mean field, i.e. a single-particle potential. The first success of the independent-particle model was the explanation of nuclear magic numbers as closed shells of single-particle states, as already discussed in Chapter 1 [May49; Hax49]. The states were obtained with a harmonic-oscillator potential including spin-orbit $(\mathbf{l} \cdot \mathbf{s})$ and centrifugal $\mathbf{l}^{2}$ terms. This approximation is enough to explain qualitatively some of the observed nuclear properties, but the inherently missing correlations caused by the $N N$ interaction are too important for the "naive" independent-particle picture to be accurate even in the case of closed-shell nuclei.

Shell model is the natural extension of the independent-particle approximation, taking into account explicitly the $N N$ interaction, but restricting itself to the nucleons outside a certain core (the so-called valence nucleons), which is considered inert, i.e. in which nucleons occupy the single-particle states with probability one, as independent particles would. Typically, a doubly-magic nucleus (both proton and neutron shells are closed) is chosen as a core. Shell model thus explicitly solves the Schrödinger equation for the part of the Hamiltonian of Eq. (3.2) corresponding to the valence nucleons. The single-particle states allow defining the inert core and the valence space (set of single-particle states which can be occupied by the valence particles) and form the basis in which the nuclear Hamiltonian is expressed prior to diagonalization.

The nuclear shell model has been extensively used to calculate the ground-state and 
excited-state properties of light up to medium-mass nuclei [Cau05]. Very popular are the so-called large-scale shell-model calculations, which are performed in valence spaces including at least all single-particle states of a certain major shell ( $s d$ [Bro06], pf [Pov01; Hon04], sdpf [Now09], to give some well-known examples). The effective interactions are usually constrained on the properties of nuclei belonging to the studied valence space. Compared to experiment, the results show in many cases a remarkable agreement, especially close to the nuclei used for the adjustment of the interaction. Still, extrapolations can differ significantly for the same valence space if different interactions are used (see [Wie13; Ste13] for some recent examples related to the $N=34$ shell closure). The quality of the obtained results depends in many cases also on the number of single-particle states included in the valence space, which can be increased only at large computational cost. For calculating medium to heavy-mass nuclei, this computational cost is very difficult to overcome, so far making large-scale calculations impossible in these regions of the nuclear chart.

During the last years, some of the monopole anomalies observed in the shell-model description, requiring ad hoc readjustments of the effective interaction, were linked to its previously disregarded three-nucleon part (see, for example, discussion in [Cau05]). First shell-model calculations with interactions derived in chiral-EFT, in which three-nucleon contributions are consistently included, have recently become available and show promising results [Bog10]. The additional numerical complications of these new approaches have nevertheless restricted their predictions so far to chains of proton-magic nuclei, such as oxygen [Ots10] or calcium [Hol12; Gal12; Wie13].

\subsection{The Hartree-Fock-Bogoliubov approach}

\subsubsection{Hartree-Fock field}

Similarly to other techniques used in theoretical nuclear physics, the Hartree-Fock (HF) method [Rin00; Ben03] was first formulated for electrons in the Coulomb potential of the atomic nucleus. The HF approach aims at approximating the exact solution to the Schrödinger equation with that resulting from an independent-particle description, but variationally optimizes the nuclear mean field which yields the single-particle states. This is possible due to the equivalence between the Schrödinger equation and the condition that the expectation value of the energy is stationary with respect to small variations of the nuclear wave function. Furthermore, considering the many-body Hamiltonian $H$ of Eq. (3.2) and its lowest eigenvalue $E_{0}$, any trial wave function $|\psi\rangle$ yields a higher energy than $E_{0}$ (see, for example, [Rin00] for proof):

$$
E_{0} \leq \frac{\langle\psi|\hat{H}| \psi\rangle}{\langle\psi \mid \psi\rangle}
$$


The variational principle is thus the starting point of the HF method, with all its extensions. In the following, only the main results of the formalism will be highlighted without proof, in order to illustrate its mathematical structure and to define some of the concepts used in actual calculations. Unless specified, these are general concepts of selfconsistent mean-field theory, which are thoroughly detailed and referenced in textbooks such as the one by Ring and Schuck [Rin00].

A common way to formulate the methods of nuclear many-body theory is by passing from the basis of position eigenvectors (coordinate representation) to the basis of eigenvectors of a single-particle operator, such as the Hamiltonian of the spherical harmonic oscillator. From the single-particle eigenvectors one can build an occupation-number representation and the associated Fock states, which form a basis for the whole space of many-body wave functions. This description benefits from the mathematical tools and theorems of the second-quantization formalism and is well suited for the HF method, the quantum states of which are Fock states corresponding to a variationally optimized single-particle basis. The description can also be rather straightforwardly translated into a numerical algorithm.

Given a basis of single-particle states, the many-body Hamiltonian of Eq. (3.2) can be written as [Rin00]:

$$
\hat{H}=\sum_{i, j} t_{i j} a_{i}^{\dagger} a_{j}+\frac{1}{4} \sum_{i, j, k, l} \bar{v}_{i j, k l} a_{i}^{\dagger} a_{j}^{\dagger} a_{l} a_{k},
$$

with

$$
\begin{gathered}
t_{i j}=\langle i|\hat{t}| j\rangle, \\
\bar{v}_{i j, k l}=\langle i j|\hat{v}| k l\rangle-\langle i j|\hat{v}| l k\rangle,
\end{gathered}
$$

matrix elements of the kinetic-energy and two-body potential between states of the singleparticle basis.

In Eq. (3.4), $a_{i}^{\dagger}$ and $a_{i}$ represent the creation and annihilation operators for the state $i$, obeying fermionic anti-commutation relations ${ }^{1}$. In the HF approach one seeks the function which minimizes the energy in the subset of antisymmetric wave-functions of independent particles, which in coordinate representation is a Slater determinant $|\phi\rangle$ of single-particle states. Using the creation operators of a single-particle basis, the Slater determinant $|\phi\rangle$ of $A$ particles corresponding to this basis can be expressed as:

$$
|\phi\rangle=\prod_{i=1}^{A} a_{i}^{\dagger}|-\rangle,
$$

where $|-\rangle$ is the particle vacuum state. Using Eq. (3.4), the expectation value of the

\footnotetext{
${ }^{1}$ The operator symbol ^ will be omitted, for simplicity, for the creation and annihilation operators
} 
energy for the a priori unknown state $|\phi\rangle$ is [Rin00]:

$$
E[\rho]=\langle\phi|\hat{H}| \phi\rangle=\sum_{i, j} t_{i j} \rho_{j i}+\frac{1}{2} \sum_{i, j, k, l} \rho_{k i} \bar{v}_{i j, k l} \rho_{l j},
$$

where

$$
\rho_{i j}=\left\langle\phi\left|a_{j}^{\dagger} a_{i}\right| \phi\right\rangle
$$

is the one-body density matrix.

One notices that in the HF case the binding energy is a functional of the nuclear density. This is the first step towards density functional theory (DFT) [Koh65], a widelyused approach to the quantum many-body problem for electron systems. In DFT, the starting point of a many-body calculation is the energy density functional (EDF) and not the many-body Hamiltonian. The existence of the EDF has been proven for systems in an external potential, as well as the fact that the density which minimizes the EDF is the ground-state density [Hoh64], which contains the full quantum-mechanical information about the many-body system. The extension of DFT to the nuclear many-body problem is nowadays a subject of great interest for theoretical nuclear-structure research, however it goes beyond the scope of the current work (see [Dob11] for a recent review from the nuclear-physics perspective). The Hartree-Fock and Hartree-Fock Bogoliubov approaches are the simplest example of DFT, in which the EDF is still derived starting from an initial Hamiltonian.

The variational condition of energy minimization with respect to the unknown Slater determinant $|\phi\rangle$ is equivalent to the minimization with respect to $\rho$, with the additional condition that $\rho^{2}=\rho$ (true when $|\phi\rangle$ is a Slater determinant):

$$
\frac{\delta}{\delta \phi}\langle\phi|\hat{H}| \phi\rangle=0 \equiv\left(\frac{\delta}{\delta \rho} E[\rho]\right)_{\rho^{2}=\rho}=0 .
$$

From Eq. (3.10) one obtains the Hartree-Fock equations [Rin00]:

$$
\sum_{j} h_{i j} D_{j k}=\epsilon_{k} D_{i k}
$$

where

$$
h_{i j}=t_{i j}+\Gamma_{i j}=t_{i j}+\sum_{l, k} \bar{v}_{i k j l} \rho_{l k}
$$

is the HF single-particle Hamiltonian and

$$
b_{j}^{\dagger}=\sum_{i} D_{i j} a_{j}^{\dagger}
$$

is the transformation from the initial single-particle basis to the eigenbasis of $\hat{h}$. Equations (3.11) and (3.12) show that the Hartree-Fock equations are an eigenvalue problem 
for the single-particle Hamiltonian $\hat{h}$, which contains the single-particle kinetic-energy $\hat{t}$ and the Hartree-Fock field $\hat{\Gamma}$. The latter term, however, depends on the density matrix, as shown in Eq. (3.12), which in turn depends on the Slater determinant $|\phi\rangle$, i.e. on the solution of the HF equations. Plugging Eq. (3.13) into Eq. (3.9), one obtains an expression for the density matrix of the solution $|\phi\rangle$ :

$$
\rho_{i j}=\sum_{k=1}^{A} D_{i k} D_{j k}^{*}
$$

which allows writing the HF equations as:

$$
\sum_{j}\left(t_{i j}+\sum_{l, k} \sum_{m=1}^{A} \bar{v}_{i k j l} D_{l m} D_{k m}^{*}\right) D_{j k}=\epsilon_{k} D_{i k} .
$$

Equation (3.15) is a self-consistent equation for the transformation from the initial to the HF basis, which is solved iteratively. According to the variational principle, the Hartree-Fock field $\hat{\Gamma}$ is the optimal approximation of the given interaction $\hat{v}_{i, j}$ to an independent-particle potential, i.e. the one which minimizes the energy. The price to pay is that generally the mean field breaks the symmetries of the nuclear Hamiltonian $H$ in a way accounting for the correlations which cannot be included explicitly in an independent-particle picture. In particular, breaking the translational symmetry accounts for the clustering of the protons and neutrons to form a finite nucleus, while breaking the rotational symmetry (deformation of the mean field) accounts for the long-range part of the $N N$ interaction, which is responsible for multipole correlations.

\subsubsection{Pairing field}

There is significant experimental evidence for the existence of pairing correlations in nuclei, such as the difference in excitation spectrum between even-even and odd nuclei, the odd-even staggering of binding energies and the moments of inertia of rotational bands. The concept of pairing was introduced in condensed matter physics, where, under the action of an attractive (effective) interaction, electrons can couple to form the so-called Cooper pairs, responsible at low temperatures for the phenomenon of superconductivity, as described by the Bardeen-Cooper-Schrieffer (BCS) theory [Bro13]. Pairing correlations are however not described by the Hartree-Fock field $\hat{\Gamma}$.

In nuclei, as shown by M.-G. Mayer [May50], the attractive, short-range part of the residual interaction energetically favors the coupling of two nucleons in the same $j$ shell to a total angular momentum $J=0$. The prerequisites for the formation of Cooper pairs are thus met in the nuclear environment, meaning that the concepts of BCS theory can be extended to the nuclear many-body problem in order to include pairing correlations. In the following, we will be referring only to the pairing between nucleons of the same 
kind ( $p p$ and $n n$ pairing, respectively). Proton-neutron $(p n)$ pairing is thought to play an important role in the case of $Z \approx N$ nuclei [Dea03], but its impact is reduced for systems with large neutron-proton asymmetry, as the ones we will mostly be referring to in this chapter.

In BCS theory, instead of the Slater determinant of Eq. (3.7), one approximates the ground-state wave function of an even-even system with the following ansatz [Rin00]:

$$
|B C S\rangle=\prod_{k>0}\left(u_{k}+v_{k} a_{k}^{\dagger} a_{\bar{k}}^{\dagger}\right)|-\rangle,
$$

where $k$ and $\bar{k}$ represent paired single-particle states and $u_{k}, v_{k}$ are occupation amplitudes $\left(\left|v_{k}\right|^{2}\right.$ is the probability that the pair $(k, \bar{k})$ is occupied and $\left.\left|u_{k}\right|^{2}+\left|v_{k}\right|^{2}=1\right)$. A common way of choosing $\bar{k}$, the conjugate of the state $k$, is as its time-reversed state.

The BCS ansatz explicitly makes pairs of single-particle states appear in the expression of the many-body wave function, which is no longer a state of independent particles. The BCS ground state is also not an eigenstate of the particle-number operator. In the BCS method, the coefficients $u_{k}$ and $v_{k}$ are variationally optimized to minimize the binding energy, starting from the many-body Hamiltonian of Eq. (3.4) and with an additional constraint on the particle number. The latter constraint ensures that the nucleus has a correct number of protons and neutrons on average. From the variational condition of energy minimization one obtains the BCS equations, which are self-consistent equations for the coefficients $u_{k}$ and $v_{k}$. The BCS formalism and resulting equations can be found, for example, in [Rin00].

What is important for the following discussion is that the BCS wave function of Eq. (3.16) can be written as a product state:

$$
|B C S\rangle \propto \prod_{k} \alpha_{k}|-\rangle
$$

where $\alpha_{k}$ are annihilation operators corresponding to a new set of states (called quasiparticle states), which are given by the following BCS transformation:

$$
\begin{aligned}
& \alpha_{k}^{\dagger}=u_{k} a_{k}^{\dagger}-v_{k} a_{\bar{k}}, \\
& \alpha_{\bar{k}}^{\dagger}=u_{k} a_{\bar{k}}^{\dagger}+v_{k} a_{k} .
\end{aligned}
$$

The quasi-particle creation and annihilation operators also obey the fermion anticommutation relations. The equivalence between Eq. (3.16) and Eq. (3.17) suggests that it is possible to describe pairing correlations while still approximating the ground state of a nucleus by a product state, provided that it is a product of (independent) quasi-particle states of BCS type Eq. (3.18). This is the object of the Hartree-Fock-Bogoliubov approach. The Bogoliubov quasi-particles are defined more generally than the BCS quasi-particles of Eq. (3.18), by the so-called Bogoliubov transformation [Rin00]:

$$
\beta_{k}^{\dagger}=\sum_{l} U_{l k} a_{l}^{\dagger}+V_{l k} a_{l}
$$


Although more general, the Bogoliubov transformation is shown by the Bloch-MessiahZumino theorem [Blo62; Zum62] to be a BCS transformation of type Eq. (3.18), performed in the basis in which the one-body density matrix is diagonal (called the canonical basis). Starting from the definition of Eq. (3.19), the ground-state wave function is then sought as the most general product state of independent Bogoliubov quasi-particles:

$$
\left|\phi_{H F B}\right\rangle=\prod_{k} \beta_{k}|-\rangle
$$

The HFB wave function of Eq. (3.20) is, as the BCS wave function, not an eigenstate of the particle number operator. It has the properties of a vacuum for the quasi-particle space (zero quasi-particle state) and thus will also be called in the following HFB vacuum. With the variational condition of energy minimization with respect to $\phi_{H F B}$ (essentially $U_{l k}$ and $\left.V_{l k}\right)$ and the additional constraint on particle number, ensuring the correct particle number on average, one obtains the HFB equations [Rin00]:

$$
\left(\begin{array}{cc}
\hat{h}-\lambda & \hat{\Delta} \\
-\hat{\Delta}^{*} & -\hat{h}^{*}-\lambda
\end{array}\right)\left(\begin{array}{l}
U_{k} \\
V_{k}
\end{array}\right)=\left(\begin{array}{l}
U_{k} \\
V_{k}
\end{array}\right) E_{k},
$$

where $\hat{h}$ is the HF single-particle Hamiltonian of Eq. (3.12), containing the HF field $\hat{\Gamma}$, $\lambda$ is the chemical potential, determined by the particle-number condition, and $\hat{\Delta}$ is the pairing field:

$$
\Delta_{i j}=\frac{1}{2} \sum_{l, k} \bar{v}_{i j l k} \kappa_{l k}
$$

with $\kappa_{l k}$ the pairing tensor:

$$
\kappa_{l k}=\left\langle\phi_{H F B}\left|a_{k} a_{l}\right| \phi_{H F B}\right\rangle .
$$

The dimensionality of the HFB equations is twice that of the HF equations. For every eigenvector $\left(U_{k}, V_{k}\right)$ of energy $E_{k}$ there is also the eigenvector $\left(V_{k}^{*}, U_{k}^{*}\right)$ of energy $-E_{k}$. The HFB wave function of Eq. (3.20) is determined by choosing one vector for each $\left(E_{k},-E_{k}\right)$ pair [Rin00]. The choice of occupied (quasi-hole) and free (quasi-particle) states is nontrivial. One common choice is to occupy the negative energy states, however, as discussed in [Ber09], this prescription does not guarantee the minimization of the energy, or the convergence of the algorithm.

Furthermore, irrespective of the average number of particles, the HFB wave function has a certain number parity, meaning that it is either a mixture of odd or even states of the particle-number operator [Ban74]. The number parity depends on the coefficients of the Bogoliubov transformation. In general, the ground state of an even-even nucleus is described by an HFB vacuum in which the negative-energy quasi-particle states are occupied, which corresponds to a proper Bogoliubov transformation (matrix of the transformation in Eq. (3.19) has the determinant equal to 1). The ground state of an odd 
nucleus is described as a single quasi-particle state created on the even HFB vacuum [Rin00; Dug01; Sch10], corresponding to the improper Bogoliubov transformation (matrix of the transformation in Eq. (3.19) has the determinant equal to -1). This is achieved in practice by replacing a quasi-particle state in the HFB wave function with the state of opposite energy. A more detailed description of this procedure and its implications can be found in [Ber09; Dob09b; Sch10]. Apart from the change of number parity of the wave function, the matrix $U$ of the Bogoliubov transformation becomes singular and the occupation amplitude of one of the single-particle states becomes equal to one. This state no longer contributes to the pairing field and in this sense it is "blocked" [Rin00].

The spectrum of quasi-particle energies is unbound from below and from above, which means that the sums defining the density matrix and pairing tensor run over an infinite number of terms. The HFB method thus requires a truncation of the quasi-particle space (see [Dob01] for an applied discussion).

\subsection{Competition between particle-particle and particle-hole correlations in nuclei}

The HFB equations of Eq. (3.21) are a generalization of the HF equations of Eq. (3.11), following the extension of the variational space from the space of HF wave functions of type Eq. (3.7) to the space of HFB wave functions of type Eq. (3.20). With this extension, apart from the one-body density matrix $\rho_{i j}$ of Eq. (3.9), the information on the many-body wave function is also contained in the pairing tensor $\kappa_{i j}$ of Eq. (3.23). The definitions of the two quantities $\rho_{i j}$ and $\kappa_{i j}$ reflect the types of correlations which are accounted for by the corresponding fields, the HF field $\hat{\Gamma}$ and the pairing tensor $\hat{\Delta}$, respectively. For a Slater determinant $\phi$ (pure HF case), the pairing tensor is null, because it reduces to the scalar product of two orthogonal Slater determinants (of different particle number). The pairing field of Eq. (3.22) is in this situation also null. The HF approximation thus accounts only for the particle-hole correlations $\left(a_{j}^{\dagger} a_{i}\right)$ through the HF field $\hat{\Gamma}$. The particle-particle correlations $\left(a_{j} a_{i}\right)$ are included in the HFB framework through the pairing field $\hat{\Delta}$.

The two types of correlations can be traced back to the different components of the $N N$ interaction. In the shell-model framework, these components can be rigorously extracted by the monopole-multipole decomposition of the nuclear Hamiltonian (see [Cau05] for a summary). The long-range part of the multipole Hamiltonian is responsible for the particle-hole correlations and can be in turn decomposed into quadrupole-quadrupole, octupole-octupole and higher multipole terms, which define the quadrupole, octupole and higher multipole correlations, respectively. The short-range, attractive part of the interaction is responsible for the particle-particle correlations (pairing phenomena). Both long-range and short-range parts of the interaction act between particles of the same kind $(p p, n n)$ or of different kinds $(p n)$. Particle-hole correlations are primarily driven by the 
pn part of interaction, because as such they are not restricted by the Pauli principle. As mentioned before, particle-particle correlations in very asymmetric nuclei (where the $N / Z$ ratio is significantly greater than 1) are predominantly driven by the $p p$ and $n n$ parts of the interaction.

As discussed in [Cas05], with respect to an initial single-particle picture resulting from a spherical mean field, the long-range part of the residual interaction favors the occupation of orbits with a specific angular relationship which maximizes its attractive effect. As such, it has a polarizing influence on the nuclear density and drives the deformation of the nuclear mean field, each mode of the mean-field deformation being connected to the corresponding multipole component of the residual interaction. Contrary to this tendency, the short-range part of the interaction favors the coupling of nucleons to pairs of angular momentum 0, the density of which has no angular dependence [Rin00]. Consequently, the effect of pairing correlations is to drive the nucleus to a spherical shape. The strength of both types of correlations is diminished close to magic numbers and becomes most significant in mid-shell regions. What results is a competition between the two contrary tendencies with the change in proton and neutron number. Close to magic numbers, particle-particle correlations dominate and nuclei maintain their spherical shape, while in the mid-shell regions of the larger valence spaces, particle-hole correlations dominate and the nuclear mean field is deformed.

In the absence of pairing correlations, all open-shell nuclei would be deformed as a manifestation of the Jahn-Teller effect [Jah37]. With pairing, however, nuclear structure goes from magic to mid-shell nuclei through a variety of intermediate states. Although simple descriptions as the so-called $N_{p} N_{n}$ scheme allow qualitatively tracing the general trends of nuclear properties [Cas87], a quantitative description requires accounting for the particular microscopic conditions. One such example is that of the neutron-rich zirconium $(Z=40)$ isotopes, the observables of which were already presented and discussed in Chapter 1 (see Fig. 1.6). Analyzing the low-lying excitation spectrum of the zirconium isotopes, it was argued that the spatial overlap of the proton and neutron orbits acts as an important catalyst of the onset of deformation through the long-range part of the proton-neutron interaction, after the filling of the $N=56$ sub-shell [Fed79]. The flat trend of the $E\left(2^{+}\right)$energies for the even-even strontium $(Z=38)$ isotopes (not peaking at $N=56$, as in the zirconium chain), was later attributed to a gradual reduction of the energy gap between the $2 p_{1 / 2}$ and $2 p_{3 / 2}$ proton single-particle levels with the addition of neutrons [Fed84], thus pointing to a fine interplay in the region between the monopole and the multipole parts of the residual interaction.

In many cases as the one mentioned above the competition between particle-particle and particle-hole correlations translates into the energy balance between a normal, spherical configuration and an intruder, deformed one [Hey11]. With the addition of nucleons in a certain open shell, the energy balance between the two configurations changes and, eventually, the intruder becomes the ground state, producing a sudden change of the 
intrinsic nuclear shape. This type of structural evolution allows interpreting shape transitions across the nuclear chart as finite-system precursors of quantum phase transitions [Cej10].

The exact position of the transition points is determined by the fine balance between the short-range, pairing interaction between like particles and the long-range, protonneutron interaction. Shape transition regions of the nuclear chart thus impose significant constraints on the modeling of the particle-particle and particle-hole channels of the nuclear potential. A too strong or too weak pairing component of the interaction would lead to the shape transition being predicted too close or too far with respect to the beginning of the nuclear shell.

\subsection{Theoretical analysis of the measured nuclear data}

\subsubsection{Aim}

In mean-field theory, the competition between particle-particle and particle-hole correlations translates into the fact that the variational problem, through the HFB equations, is solved while optimizing simultaneously the shape of the nuclear mean-field and the BCS-type occupation coefficients of the Bogoliubov transformation. The equilibrium configuration (shape) is the optimal solution with respect to the constraints coming both from the particle-particle and particle-hole channels of the nuclear interaction. Most calculations in mean-field theory use effective interactions specially tailored for nuclearstructure calculations, of which Skyrme-type and Gogny-type interactions are nowadays most widely used [Rin00]. The parameters of these interactions are usually adjusted using the masses and radii of doubly-magic nuclei, as well as a certain choice of saturation properties for symmetric nuclear matter, compatible with the properties of finite nuclei, such as the matter and energy density, symmetry energy and compressibility [Ben03]. Doubly-magic nuclei are popular choices because the closure of both proton and neutron shells eliminates the different types of correlations. The resulting interaction parameters are then not biased due to the accuracy with which these correlations are modeled in mean-field theory.

Some authors however perform a global fit of the interaction parameters on the entire set of measured nuclear masses [Gor13]. While a non-selective fit to all known nuclear binding energies gives less control on the way specific nuclear structure phenomena are reflected in the parameters of the nuclear interaction, constraining the interaction only to magic or doubly-magic nuclei disregards most of the available nuclear data. The binding energies of most nuclides can be algebraically extrapolated following the trends of their isotopes and isotones, with an accuracy better than $500 \mathrm{keV}$, which means that most nuclei would in any case not add any significant constraint on the parameters of the nuclear interaction. However, nuclei in shape transition regions are very interesting candidates, 
because their properties show discontinuities of similar strength as shell effects.

The common agreement on the mean-field description of mid-shell nuclei is that it is incomplete. The restoration of the symmetries broken at the mean-field level and the description of collective fluctuations (configuration mixing) are necessary steps for a complete description of the binding energy [Ben03; Ben06]. Symmetry restoration is also required for the description of excited states and transition probabilities [Del10; Rob11]. These extensions demand considerable numerical effort and are currently restricted to even-even nuclei. Nevertheless, the mathematical steps now constituting the "beyondmean-field" approaches ameliorate the description of the total nuclear binding energy, but for nuclei which are statically deformed they sometimes make little difference at the level of the differential mass quantities, such as the two-neutron separation energy.

The situation is not the same for nuclei close to magic numbers, which are vibrational and are not well approximated by a static spherical or deformed mean field. For these nuclei, the contribution of shape fluctuations to all observables is significant. A relevant example are the two-neutron shell gaps, which are computed using the binding energy of nuclei which are either semi-magic or only two nucleons away from a magic chain. For this quantity, only a full beyond-mean-field treatment can capture all the features of the experimental trends, as shown in [Ben06; Ben08].

Many of the nuclei in shape-transition regions, such as the neutron-rich $A \approx 100$ nuclei and the neutron-deficient gold nuclei, are very well deformed around mid-shell, which allows obtaining a reasonable description of their differences of binding energies and charge radii already at the deformed mean-field level. Given also the sharp signatures in the trends of their ground-state properties at the shape-transition point, the comparative study of these nuclei in the HFB approach imposes additional constraints on the modeling of the particle-hole and particle-particle channels of the energy functional.

In particular, given the competition between the two types of correlations on defining the evolution of nuclear configurations with proton and neutron number, the balance between the two distinctive parts of the energy functional is expected to influence decisively the predicted boundaries of the different "islands of deformation" observed across the nuclear chart. For a certain interaction giving the particle-hole part of the energy functional, the correct description of the boundaries of the shape-transition regions could narrow significantly the window of values in which the pairing interaction, giving the particle-particle part of the energy functional, can take its values. The same comment effectively holds in the framework of the energy-density-functional theory, where the different parts of the energy functional are not derived from an initial interaction.

In mean-field theory, the pairing interaction (or energy functional) is fitted to reproduce primarily the odd-even staggering of binding energies around certain magic nuclei [Ben03]. This uses the interpretation of the odd-even staggering as the effect of the blocking of pairing correlations in odd-nucleon systems, which leads to less binding on average than in the fully paired, even neighbors. In this view, the magnitude of the odd-even 
staggering scales with the strength of the pairing interaction and provides a reference for constraining it. However, as pointed out in several articles [Rut99; Ben00; Dug01] the odd-nucleon breaks time-reversal invariance and, due to its finite multipole moment, exerts a polarization effect on the even-even core. These additional effects also contribute to the predicted odd-even staggering of binding energies and, as such, make the connection between the empirical estimators of the pairing gap and the pairing interaction not so straightforward. Additional constraints on the pairing interaction, not sensitive to these effects, are thus necessary.

The rubidium and gold isotopes which have been studied with the ISOLTRAP mass spectrometer as part of this work belong to two of the most well pronounced shapetransition regions of the nuclear chart, the former situated on its neutron-rich and the latter on its neutron-deficient side. Due to their large valence spaces and many valence nucleons, the structure of the studied isotopes is difficult to access through shell-model calculations, thus leaving the self-consistent mean-field framework as the method of choice for a fully-microscopic study. The following analysis will be limited to HFB calculations with Skyrme forces, for which the particle-hole and the particle-particle parts of the energy functional are modeled separately.

The aim of the study will be two-fold. In a first instance, the different equilibrium configurations for the HFB quasi-particle vacua (Eq. (3.20)) characterizing the nuclei in the two shape transition regions will be calculated. The mean-field description of the twoneutron separation energies and charge radii for the two sets of nuclei will be compared to the available experimental data and the particularities of their trends will be interpreted through the energy balance between the determined configurations. This energy balance will then be systematically studied, for a given Skyrme parametrization, with respect to the strength of the pairing interaction, analyzing the way the latter influences the description of the shape-transition phenomenon. Finally, the one-quasi-particle states will be calculated for the odd- $N$ isotopes of selected isotopic chains. This will allow putting the pairing interaction to the severe test of consistently describing the odd-even staggering of binding energies and the boundaries of the islands of nuclear deformation.

\subsubsection{HFODD code}

The calculations have been performed using the HFODD code [Dob09b; Sch12], which solves the HFB equations in a deformed harmonic-oscillator (HO) basis. The code allows calculations unrestricted by the time-reversal or spatial symmetries, as well as choosing $\mathrm{HO}$ bases of almost arbitrarily complex deformation, to accommodate the description of triaxial, reflection asymmetric or even more complex shapes. Extensive documentation of the code is given in the User's Guide [Dob09a]

The calculations have been performed using the SLy4 parametrization (of the Skyrme family) for the particle-hole part of the energy functional [Cha98]. The choice of this interaction is based on its extensive use for the description of the properties of nuclei across 
the entire nuclear chart, from global calculations including particle/angular-momentum projection and configuration mixing for systematic studies of quadrupole correlations in even-even nuclei [Ben06], to systematic studies of pairing interactions for the particleparticle part of the energy functional [Dob01], to explorations of the limits of the nuclear chart both in the super-heavy region [Cwi05] and towards the drip lines [Erl12]. All these and other existing results constitute a solid reference basis for discussing the calculations which will be presented in this work.

A usual choice for the pairing interaction, responsible for the particle-particle part of the energy functional, is the density-dependent delta interaction (DDDI) [Dob01; Ben03]:

$$
V_{\text {pair }}\left(\mathbf{r}_{1}, \mathbf{r}_{\mathbf{2}}\right)=V_{0}\left\{1-\left[\frac{\rho\left(\mathbf{r}_{\mathbf{1}}\right)}{\rho_{c}}\right]^{\beta}\right\} \delta\left(\mathbf{r}_{1}-\mathbf{r}_{\mathbf{2}}\right),
$$

where $\rho\left(\mathbf{r}_{\mathbf{1}}\right)$ is the nuclear density. Equation (3.24) depends on three parameters. The parameter $V_{0}$ gives the overall strength of the pairing interaction, while the parameters $\rho_{c}$ and $\beta$ define the way the pairing strength is distributed as a function of the nuclear density. Given the difficulties of constraining all three parameters based on the available nuclear data, a common choice for $\beta$ is 1 [Ben03]. In this case, different types of pairing can be obtained, depending on the value of $\rho_{c}$. For $\rho_{c}=0.16 \mathrm{fm}^{-3}$, the nuclear saturation density, Eq. (3.24) will be peaked at the nuclear surface, making it a surface-type pairing. For $\rho_{c} \rightarrow$ $\infty$, the density dependence disappears and one obtains a volume-type pairing. Finally, for any intermediate situation, the pairing is of mixed type. Following the systematic study of [Dob01], the authors propose a mixed surface-volume pairing with a parameter $\rho_{c}=0.32 \mathrm{fm}^{-3}$. For the following analysis, a volume-type pairing will be used, which takes Eq. (3.24) to the simple form:

$$
V_{\text {pair }}^{\text {val }}\left(\mathbf{r}_{1}, \mathbf{r}_{2}\right)=V_{0} \delta\left(\mathbf{r}_{1}-\mathbf{r}_{2}\right) .
$$

Equation (3.25) has the advantage of containing only one adjustable parameter which defines the pairing strength. This allows analyzing the effect of the pairing interaction on the chosen set of nuclear observables, while avoiding a multiparametric problem. Furthermore, as shown in a recent paper for the tin isotopic chain [Hak12], the surface-type pairing significantly overestimates the odd-even staggering of binding energies, but the volume and mixed-type pairing give similar predictions.

The energy cut-off defining the pairing-active space is chosen to be $60 \mathrm{MeV}$. The calculations perform the one-body center-of-mass correction before variation and the LipkinNogami corrections after variation (see [Sch12] and the references therein for more details). The symmetries of the problem are chosen such that multipole deformations of even and also odd order are possible (quadrupole, octupole, hexadecapole etc.), specifically the simplex symmetry is enforced, while the signature and parity symmetries are relaxed. Except for the cases chosen to study the odd-even staggering of binding energies, odd nuclei are computed only at the level of the HFB quasi-particle vacuum. This is also called 
the "false-vacuum approximation" [Dug01; Sch10], the wave-function of the odd nucleus having even number parity, but an odd number of particles on average. One allows this simplification because the observables of interest are the two-neutron separation energies and the charge radii, the average trends of which are well described by the quasi-particle vacuum solution. In particular, as was shown in Eq. (1.16), the two-neutron separation energies filter out the odd-even staggering up to its first derivative with neutron number, meaning that the error on $S_{2 n}$ in the false-vacuum approximation rises to the value of this derivative. In reality, even for the most pronounced cases, as the one shown in Fig. 1.3, the residual staggering is small compared to the absolute value of $S_{2 n}$. Considering also that the effect of varying the strength of the pairing interaction is much larger than these small corrections and the significant gain in computation time, the false-vacuum approximation is used to study the general $S_{2 n}$ and charge-radii systematics. Still, there are certain limitations to this approximation, which will be discussed in Chapter 4 .

A complete description of odd nuclei requires a blocking prescription. In the case of the self-consistent quasi-particle blocking described in Section 3.3.2, the wave function of an odd nucleus is defined as a one-quasi-particle state created from the HFB vacuum [Dug01; Sch10]. The procedure is described in more detail in [Dob09b] and uses the "overlap approximation". At each iteration, the quasi-particle to be created is chosen as the state having the largest overlap with a certain single-particle state, which is specified at the beginning of the calculation. Several different calculations of the same nucleus are required, each using a different single-particle state for the overlap criterion. At the end, the ground state of the odd nucleus is chosen as the solution of lowest energy. This procedure will be applied in the following to calculate the odd- $N$ mercury isotopes, thus allowing to determine the finite-difference estimators of the "blocking gap" (also called "pairing gap") and compare them to experiment. These calculations allow the breaking of time-reversal symmetry in the odd nuclei.

\subsubsection{Tests of the method}

The HFB algorithm converges to an equilibrium solution which is a local minimum in energy with respect to small variations of the nuclear wave function (for example, along a certain deformation mode, such as the quadrupole one) [Rin00]. In most cases several such minima exist, usually differing by the value of their intrinsic quadrupole moment $Q_{20}$. It is often possible to calculate a spherical, an oblate $\left(Q_{20}<0\right)$ and a prolate $\left(Q_{20}>0\right)$ equilibrium configuration, but it is not uncommon for more than one oblate or prolate minimum to exist. In the most general case the number of local minima in energy can be significant, even taking only the quadrupole degree of freedom into account. They can all be mapped by performing constrained HFB calculations, i.e. adding an extra term to the energy functional which depends on the geometric moment of the deformation mode of interest and is minimum when the moment has the constrained value [Rin00]. In the case of unconstrained calculations, the HFB algorithm converges to any of the possible 
local minima, depending strongly on the initial wave function which starts the iterations and the shape of the HO basis.

The calculations performed in the present work were unconstrained, each run being started with the eigenfunctions of a Nilsson potential matched to the chosen HO basis [Dob09a]. Two different numerical approaches were tested. For the first approach, a spherical HO basis was used. In this case, the starting wave function was spherically symmetric and, due to self-consistency, the whole problem. The mean field and the resulting wave function in every subsequent iteration was also spherically symmetric, leading, up to numerical instabilities, to a spherical solution. To obtain convergence towards one of the deformed configurations, the first ten iterations of each run were constrained to a multipole moment compatible with the desired equilibrium configuration, leaving the rest of the iterations unconstrained until convergence was reached. For the second approach, the deformation of the $\mathrm{HO}$ basis was systematically changed in the deformation mode of interest for the equilibrium configuration. Each nucleus was computed on a set of different HO bases, with starting conditions matched to each basis through the corresponding Nilsson potential. For each set, the solution lowest in energy was chosen.

For both methods, only the quadrupole degree of freedom was investigated, as it is the main deformation mode of the nuclear mean-field. The oblate and prolate equilibrium configurations were considered separately, in order to study their energy balance along the different isotopic chains. For the first method, this means that only the quadrupole moment $Q_{20}$ was constrained to either a negative or positive value during the first ten iterations, driving convergence in the spherical basis towards an oblate or prolate solution, respectively. For the second method, only the quadrupole deformation parameter of the HO basis was systematically varied between negative and positive values. Oblate/prolatedeformed bases with corresponding starting conditions lead to oblate/prolate equilibrium configurations.

The advantage of working in a HO spherical basis is that the trends of the computed ground-state properties along an isotopic chain are not influenced by the sudden change of optimal basis shape from one isotope to the next. This can in principle be the case when a set of deformed HO bases is used. The variation of the energy of the same configuration over the whole range of bases can be as high as a few hundred keV, which requires a sufficiently fine sampling of the basis deformation, in order to find for each isotope a basis close to the optimal and to reduce the isotope-to-isotope numerical noise. The disadvantage of using a spherical HO basis is the high dependency of the successful convergence to the deformed configurations on the conditions obtained at the end of the constrained iterations. Typically, in the spherical basis several calculations still have to be performed for each nucleus, varying the value of the quadrupole moment to which the first iterations are constrained, in order to achieve proper convergence to the desired configurations. In the case of solutions with low deformation or of higher energy than the spherical one, convergence can even fail systematically for many different initial conditions. 
This problem is particularly inconvenient for the study of shape transition regions, because at the transition point one always finds two or even more configurations lying very close in energy and even deformation, making the exact location of the shape transition sometimes difficult to establish.

Mainly due to the latter difficulty and due to the generally improved stability rate of the algorithm when using deformed HO bases, the latter method was chosen for the extended range of calculations. Nevertheless, in order to test the consistency of the results obtained by the two methods and to eliminate potential spurious effects, both methods were applied to the study of a representative subset of the neutron-rich $A \approx 100$ and neutron-deficient gold-thallium nuclei. In the following, the results obtained for the krypton and gold isotopes by the two methods will be presented for comparison. All calculations in this chapter are obtained in a (spherical or deformed) HO basis containing around $300 \mathrm{HO}$ states. Because the code does not consider the degeneracy of shells when counting the number of states in the basis, the actual number of states included is slightly different for the spherical HO $(\approx 290)$ and for the deformed HO $(\approx 305)$. Still, the difference is small enough that it does not significantly impact the predicted energy balance between the deformed and the spherical solutions.

As a test of the numerical method, Fig. 3.1 shows the deformation energy (difference in binding energy between the deformed and the spherical solution) of the equilibrium configurations along the krypton and gold isotopic chains, obtained at the level of the HFB quasi-particle vacuum (no blocking). Positive values mean that the deformed configuration is more bound than the spherical one. The strength $V_{0}$ of the pairing interaction is set to a starting value of $V_{\pi}=V_{\nu}=-200 \mathrm{MeV} \mathrm{fm}^{3}$, the same for protons and neutrons. One notices that the krypton and gold isotopes exhibit both types of equilibrium shapes (oblate and prolate). If more than one equilibrium prolate/oblate configuration is found for a certain nucleus, Fig. 3.1 shows the value of lowest energy.

Although the difference in energy between the same configuration computed in a spherical and in a deformed basis can be as large as $1 \mathrm{MeV}$, this difference doesn't vary much from one isotope to the next. This means that for the same configuration $S_{2 n}$ only differs by a small amount between the different basis choices. However, both in the krypton and in the gold isotopic chains one observes the competition in energy between the prolate and oblate configurations, of which the one lowest in energy (in Fig. 3.1 the one highest in deformation energy) gives the ground state in the mean-field approximation. Around mid-shell $(N>60$ for krypton and $N<105$ for gold) the two configurations come very close in energy. At this point, their energy balance becomes sensitive to the quality with which each configuration is described in a spherical or in a deformed basis.

For the example of Fig. 3.1(a), the oblate and prolate configurations are almost degenerate for $N<56$ and $N>60$ in the spherical HO basis, but the oblate configuration is systematically more bound than the prolate one if the deformed HO bases are used. Similarly, in Fig. 3.1(b) the difference in energy between the prolate and the oblate solutions 


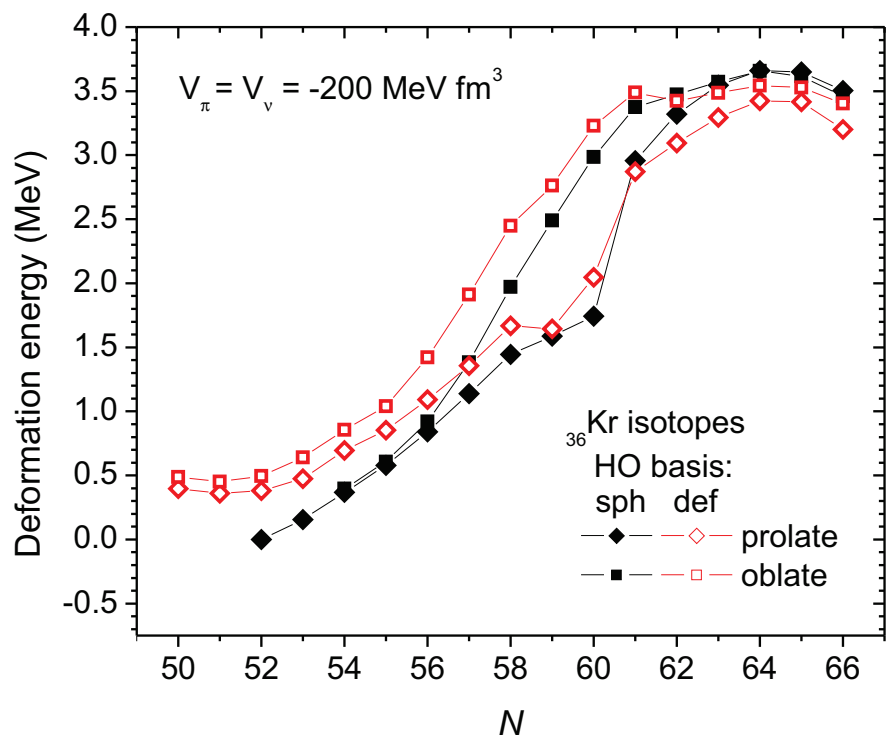

(a)

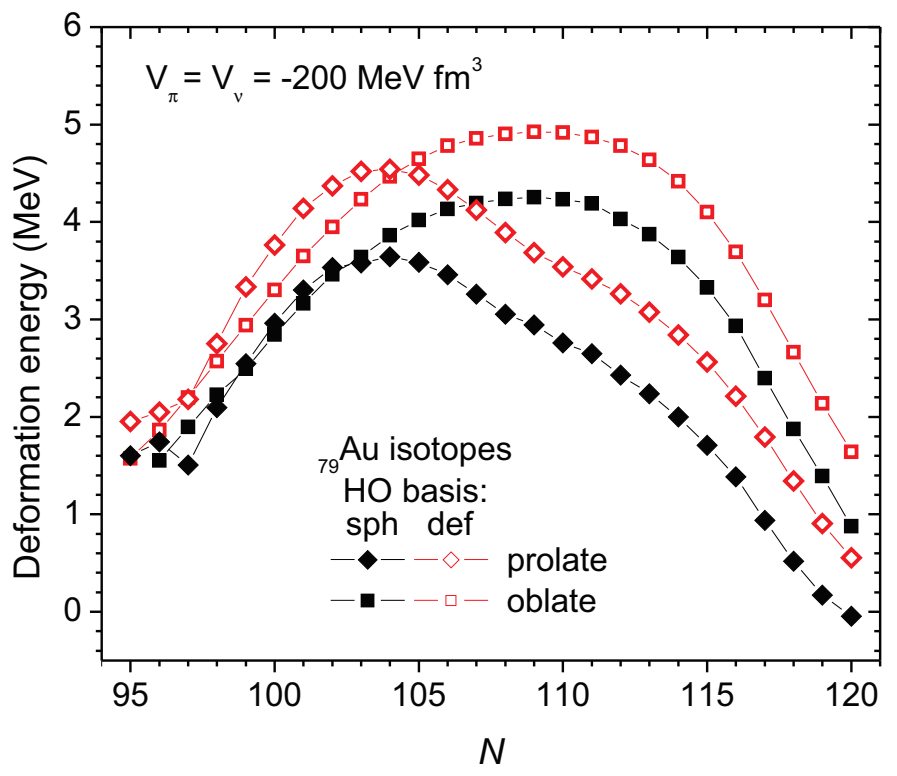

(b)

Figure 3.1: Calculated deformation energy of the prolate (diamonds) and oblate (squares) equilibrium configurations of the HFB quasi-particle vacuum obtained using a spherical HO basis (full black symbols) and a set of deformed HO bases (empty red symbols). The results are shown for the krypton (a) and gold (b) isotopes. 
below $N=105$ is significantly lower in the spherical basis.

The mean-square charge radii of the prolate configurations along the krypton isotopic chain and of the oblate configurations along the gold isotopic chain are shown in Fig. 3.2 for different choices of HO basis, starting with a spherical basis and then going through deformed bases of increased quadrupole deformation parameter $\beta_{20}$. In the case of the deformed bases the solution of minimum energy is also highlighted. The radii are presented, as will be the case throughout this work, as mean-squared displacements from the value of a reference isotope ( $N=50$ for the $A \approx 100$ nuclides and $N=115$ for the gold-thallium nuclides): $\left\langle\delta r^{2}\right\rangle_{N, r e f}=\left\langle r_{N}^{2}\right\rangle-\left\langle r_{r e f}^{2}\right\rangle$. For both isotopic chains, the calculated charge radii slightly increase with the deformation of the HO basis (in Fig. 3.2 the displacement $\left\langle\delta r^{2}\right\rangle_{N, r e f}$ is calculated with respect to the charge radius of the minimum-energy solution obtained for the reference isotope). Because, as can be seen in Fig. 3.2, the increase also affects the reference isotope, the change of basis shape would not significantly affect $\left\langle\delta r^{2}\right\rangle_{N, r e f}$.

For the prolate solutions of the krypton isotopes, a second equilibrium configuration (energy minimum), of higher deformation (and charge radius), emerges around $N=$ 60. Depending on the deformation of the HO basis, the algorithm converges to one or another configuration. Similarly, in a spherical basis the result depends strongly on the starting conditions. For the example of Fig. 3.2, the calculation in a spherical basis misses the solution of higher charge radius at $N=60$, despite several trials with different starting conditions. This can happen around shape-transition points, making calculations in deformed $\mathrm{HO}$ bases a more accurate method of sampling the spectrum of deformed configurations.

The results presented in Figs. 3.1 and 3.2 thus show that the present approach should be quite reliable in tracing the trends of differential mass quantities and of relative meansquare charge radii $\left\langle\delta r^{2}\right\rangle$ for each equilibrium configuration, the results being quite robust with respect to the deformation of the HO basis. A similar robustness of the differential mass quantities with respect to the variation of the size of the HO basis was also reported [Dob97]. However, the energy balance between the competing configurations is sensitive to the choice of basis, making their exact ordering uncertain in the immediate vicinity of shape-transition points. Considering only the comparison between the calculations in a spherical and in a deformed basis, the basis-choice uncertainty can lead to the shape transition being predicted one neutron number earlier or later, depending on the preferred method. This uncertainty is, as will be shown, significantly lower than the effects of the pairing interaction on the evolution of the different configurations with proton and neutron number. The obtained precision is thus sufficient for the requirements of the present study. For an exact adjustment of the pairing interaction, however, one must systematically study the effect of the basis size and shape on the predicted values of the reference observables. Otherwise, a large basis can be employed, which reduces the sensitivity of the calculation to the basis shape [Dob97]. 


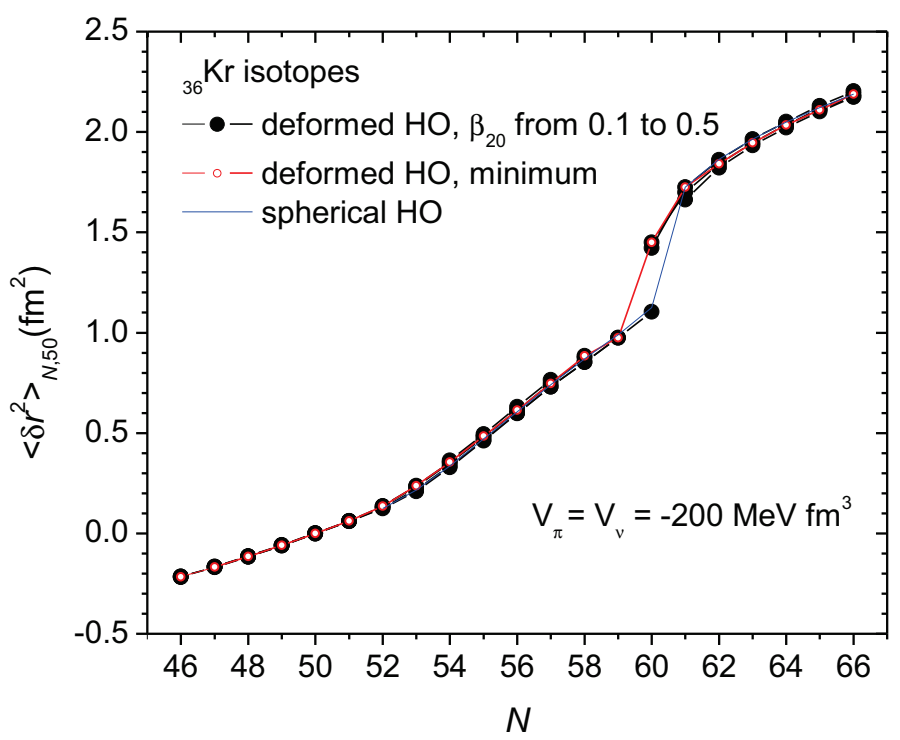

(a)

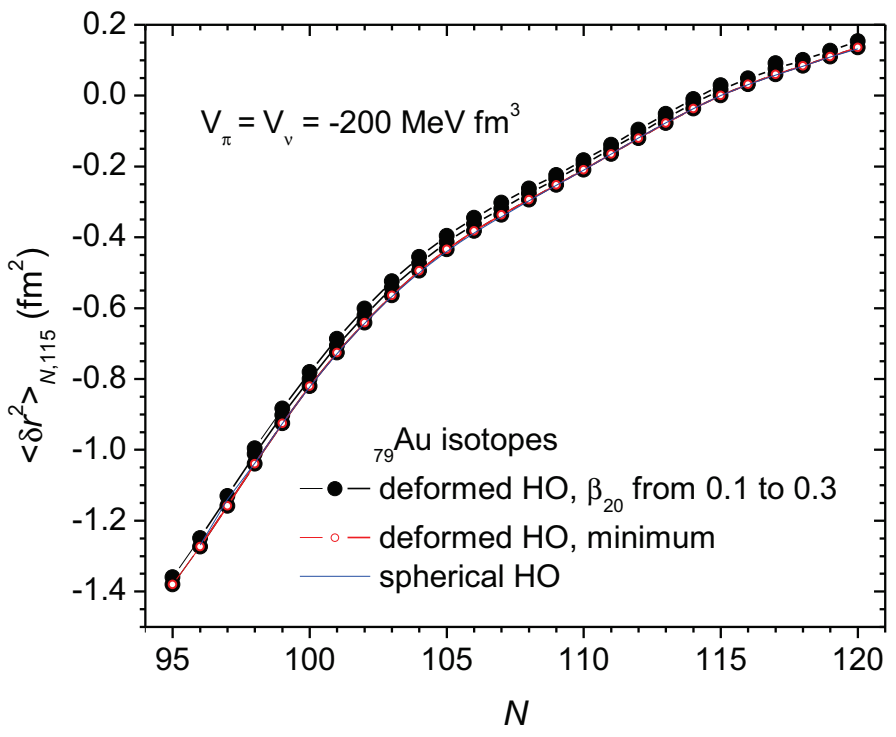

(b)

Figure 3.2: Theoretical mean-square charge radii of the prolate configurations along the krypton isotopic chain (a) and oblate configurations along the gold isotopic chain (b), plotted as displacements to a reference isotope: $\left\langle\delta r^{2}\right\rangle_{N, r e f}=\left\langle r_{N}^{2}\right\rangle-\left\langle r_{r e f}^{2}\right\rangle\left(N_{\text {ref }}=50\right.$ for krypton and $N_{\text {ref }}=115$ for gold). The results are shown for calculations in a spherical HO basis (blue thick line) and in deformed bases of increase quadrupole deformation $\beta_{20}$ (black full circles). The results obtained in the deformed basis which yields the lowest binding energy are marked for each isotope by a red empty circle. 
Although several basis shapes were systematically tested for each isotope in the two studied regions of the nuclear chart, the optimal shape was, with few exceptions, the same for a certain isotopic chain, pairing strength and nuclear configuration (e.g oblate, low-deformation prolate, high-deformation prolate). This outcome significantly reduced the numerical noise of the obtained results. 


\section{Chapter 4}

\section{Self-consistent mean-field calculations}

\subsection{Neutron-rich $A \approx 100$ nuclei}

The HFB approach using the SLy4 functional [Cha98] and a volume contact pairing interaction Eq. (3.25) was first applied to describe the ground-state masses and charge radii of the neutron-rich $A \approx 100$ nuclei in the isotopic chains between krypton and zirconium. These nuclei have been studied extensively by different approaches, from shell model [Fed79; Fed84; Hey87], to the interacting boson model (IBM) [Gar05] and the selfconsistent mean-field approximation [Rod10a; Rod10b]. The properties of the nuclei in the region can also be extracted from global mean-field calculations, spanning the entire nuclear chart (the Skyrme-HFB calculations of [Gor13] achieving the lowest root-meansquare deviation to the measured masses), of which some include angular-momentum projection and configuration mixing for the even-even nuclei ([Ben06] using an HFBSLy4 approach and [Del10] using the Gogny-D1S interaction). Global calculations for even-even nuclei including the octupole degree of freedom have also been published, using Gogny-type interactions [Rob11].

In agreement with the existing body of theoretical work, the calculations presented here predict that the neutron-rich $A \approx 100$ nuclei possess both oblate and prolate equilibrium configurations of the nuclear mean-field, in competition with each other and the spherical one. A single oblate configuration was found in each of the studied isotopic chains, having a smooth variation with neutron number. Two distinct prolate configurations were however identified, one of lower quadrupole deformation (and lower charge radius) which is already present close to $N=50$ and one of high quadrupole deformation (and larger charge radius) which emerges around $N=60$.

To illustrate how the strength of the pairing interaction influences the spectrum of deformed configurations, Fig. 4.1 shows the deformation energy of the oblate and (most 


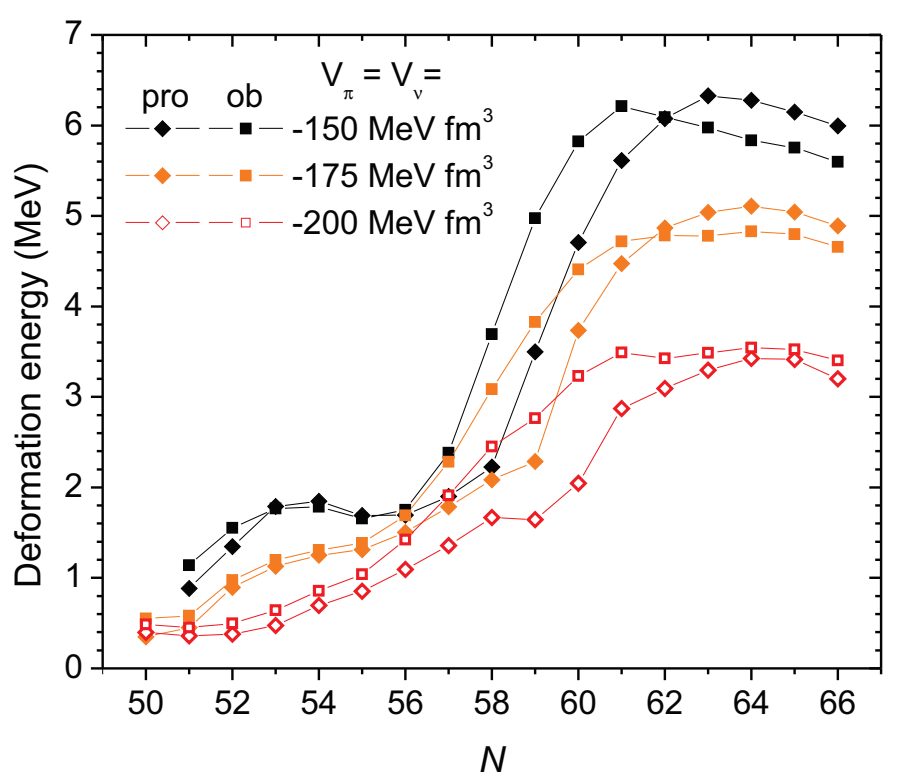

Figure 4.1: Deformation energy of the prolate and oblate equilibrium configurations (diamonds and squares, respectively) of the krypton isotopes, obtained at the level of the HFB quasi-particle vacuum, using different values of the pairing interaction. For each isotope, the minimum-energy prolate and oblate configuration is represented.

bound) prolate configuration along the krypton isotopic chain, for values of the pairinginteraction parameter, $V_{0}$ in Eq. (3.25), ranging from $-150 \mathrm{MeV} \mathrm{fm}^{3}$ to $-200 \mathrm{MeV} \mathrm{fm}{ }^{3}$. Equal strengths are chosen for the proton and neutron pairing interaction to preserve isospin symmetry, although it is not uncommon to use different strengths [Ben03]. Odd nuclei are calculated only at the level of the HFB vacuum (no blocking).

One notices in Fig. 4.1 that increasing the strength of the pairing interaction reduces the deformation energy of both the prolate and the oblate minima. The amount of this increase is however different for the competing configurations. For $V_{0}=-150 \mathrm{MeV} \mathrm{fm}^{3}$, the prolate solution becomes significantly more bound than the oblate one for $N>62$, while for $V_{0}=-200 \mathrm{MeV} \mathrm{fm}^{3}$ the oblate solution is more bound for every neutron number.

To understand how this evolution affects the ground-state observables, Fig. 4.2 shows the experimental and theoretical $S_{2 n}$ and $\left\langle\delta r^{2}\right\rangle$ values of the neutron-rich $A \approx 100$ nuclei in the isotopic chains between krypton $(Z=36)$ and zirconium $(Z=40)$. When estimated by the authors, the error bars in the $\left\langle\delta r^{2}\right\rangle$ plot show the full systematic uncertainties of the measurements. Apart from the krypton isotopes, all $S_{2 n}$ and $\left\langle\delta r^{2}\right\rangle$ values are shifted upward (experimental and theoretical by the same value) for better separation. The calculated $S_{2 n}$ and $\left\langle\delta r^{2}\right\rangle$ are plotted separately for the (HFB vacuum of the) oblate and prolate configurations of the studied isotopes. In the case of two coexisting prolate 
minima, the one of lower energy is used. The trends of the results obtained in the spherical approximation are also shown for reference. This allows observing explicitly the effect of deformation on the studied observables. The ground state of each nucleus is assigned to the equilibrium configuration of lowest energy and the resulting $S_{2 n}$ and $\left\langle\delta r^{2}\right\rangle$ values are marked explicitly as the "global minimum".

The mean-square charge radii allow tracing very clearly the evolution of the different mean-field configurations and their energy balance. To also interpret the trends of the twoneutron separation energies, one can use a similar approach to the analysis of Chapter 1. In analogy to Eq. (1.3), the binding energy can be written as:

$$
E(Z, N)=E_{s p h}(Z, N)+\frac{(-1)^{N+1}+1}{2} \delta_{\nu}+\frac{(-1)^{Z+1}+1}{2} \delta_{\pi}-E_{\text {def }}(Z, N),
$$

where $E_{s p h}$ is the energy of the $(Z, N)$ nucleus obtained at the end of a HFB calculation in the spherical approximation, $\delta_{\nu}\left(\delta_{\pi}\right)$ represents the binding energy "lost" by the odd- $N$ (odd- $Z$ ) nucleus with respect to the fully-paired HFB vacuum when blocking is implemented (in the present case by creating a one-quasi-particle state) and $E_{\text {def }}$ represents the deformation energy (plotted in Fig. 4.1 for the krypton isotopes), i.e. the binding energy "gained" by a certain deformed configuration with respect to the spherical solution. Because $\delta_{\nu}$ and $\delta_{\pi}$, as defined above, determine an odd-even staggering of the nuclear binding energies, it is common to interpret the odd-even staggering phenomenon as the result of the blocking of pairing correlations in odd-nucleon systems. Both $\delta_{\nu}, \delta_{\pi}$ and $E_{\text {def }}$ are positive quantities in Eq. (4.1). In calculations without quasi-particle blocking (even-even systems or false-vacuum solutions of odd systems) such as the ones shown in Fig. 4.2, the $\delta_{\nu}, \delta_{\pi}$ terms are not present and Eq. (4.1) reduces to:

$$
E(Z, N)=E_{s p h}(Z, N)-E_{d e f}(Z, N) .
$$

Calculating $S_{2 n}$ from Eq. (4.2) leads to the expression:

$$
S_{2 n}(Z, N)=\Delta_{2, N} E(Z, N)=S_{2 n, s p h}(Z, N)+E_{d e f}(Z, N)-E_{\text {def }}(Z, N-2),
$$

which is similar to Eq. (1.17), the difference being that the spherical solution includes the effect of pairing correlations (it is not an independent-particle problem) and thus it is not possible to express $E_{\text {sph }}(Z, N)$ by the sum of single-particle energies of Eq. (1.4). Equation (4.3) illustrates the effect of the onset of deformation on the two-neutron separation energies: an increase of the deformation energy in the nuclear ground state leads to a higher $S_{2 n}$ than the spherical trend. The "spherical trend" is not an observable quantity, but along an isotopic chain it can be inferred based on the $S_{2 n}$ slope close to the beginning of the shell. Approaching mid-shell, the gradual increase of $E_{\text {def }}$ is observed as a flattening of the $S_{2 n}$ trend or, in extreme cases in which $E_{d e f}(Z, N)$ has a sudden increase with the addition of a single neutron, as an increase of $S_{2 n}(Z, N)$ with respect to $S_{2 n}(Z, N-1)$. 


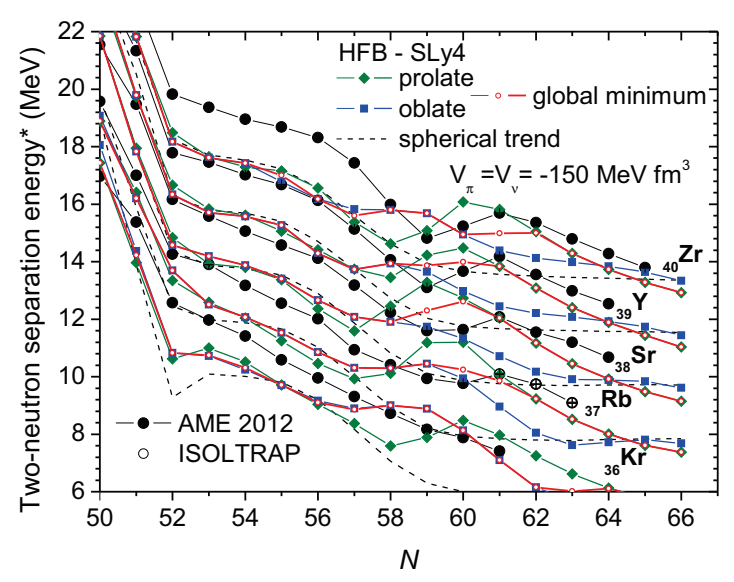

(a)

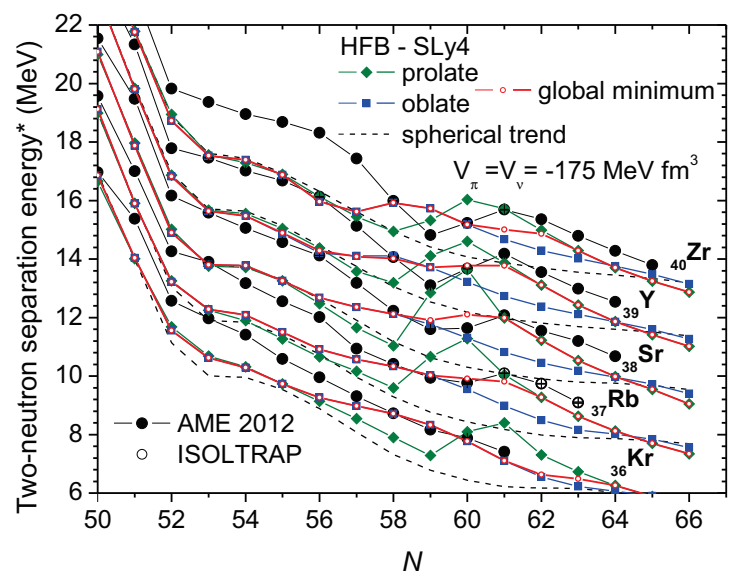

(c)

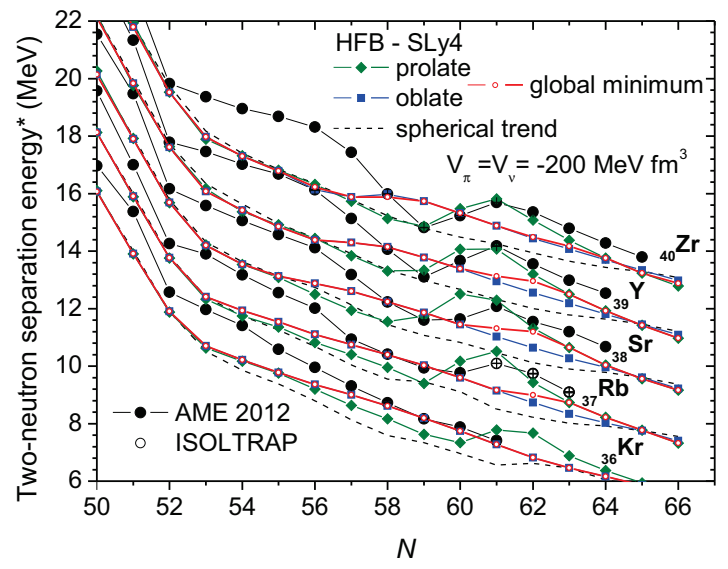

(e)

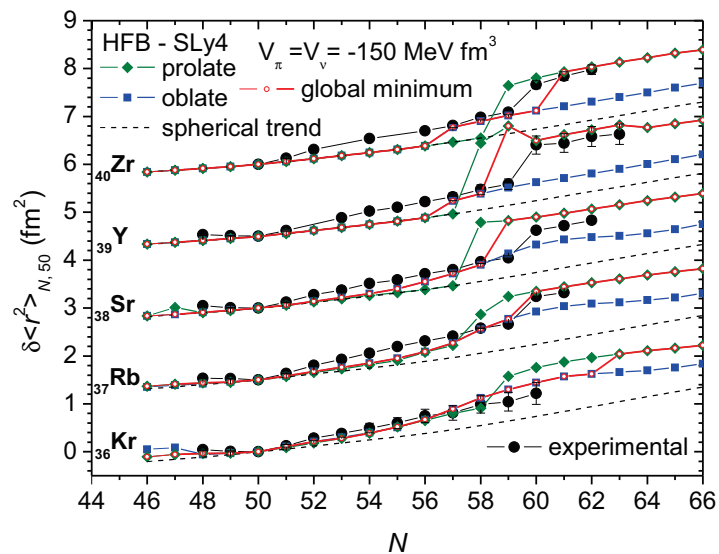

(b)

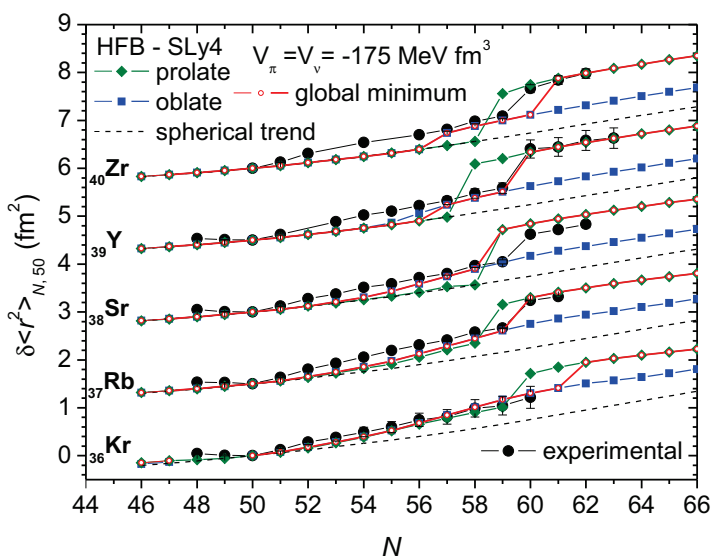

(d)

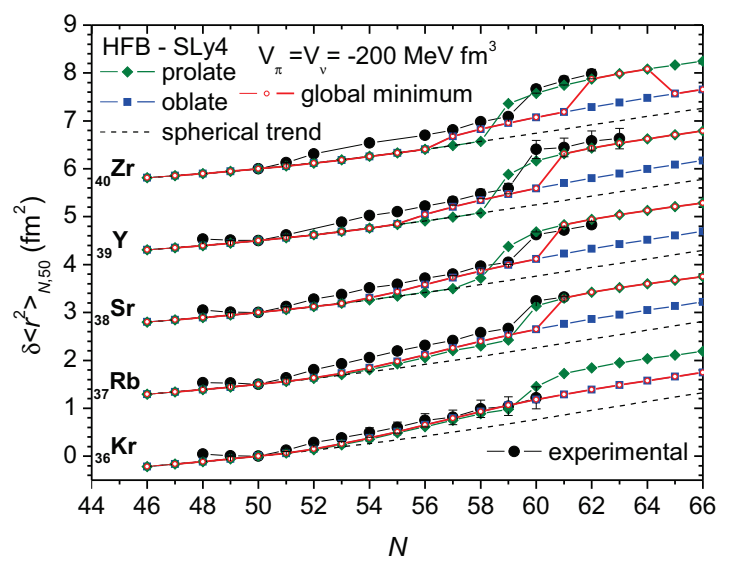

(f)

Figure 4.2: Experimental two-neutron separation energies and mean-square charged radii of neutron-rich $A \approx 100$ nuclei, compared to HFB-SLy4 calculations using three strengths of the pairing interaction. The experimental $S_{2 n}$ values use masses from the AME2012 [Wan12] and the present work, published in [Man13]. The $\left\langle\delta r^{2}\right\rangle$ values are taken from [Kei95; Thi81; Buc90; Lie91; Che07; Cam97; Cam02; Tha03]. The theoretical values represent the HFB quasi-particle vacuum (no blocking) of the spherical, lowest-energy oblate and lowest-energy prolate configurations (dashed line, blue squares and green diamonds, respectively). The red empty circles represent the values corresponding to the ground state, assigned to the configuration of lowest energy. Except for the krypton chain, the $S_{2 n}$ and $\left\langle\delta r^{2}\right\rangle$ values are biased for better separation. 
Because the deformation energy can vary abruptly around the borders of shape-transition regions (see the prolate solutions of Fig. 4.1 around $N=60$ ), the systematics of $S_{2 n}$ can contain clear signatures indicating the occurrence of a shape transition.

With these observations, the two-neutron separation energies and mean-square charge radii of Fig. 4.2 can be readily interpreted. Irrespective of the value of the pairing interaction, the prolate and oblate equilibrium configurations have two qualitatively distinct evolutions with neutron number. The oblate configuration exhibits a smooth onset of deformation. Of the prolate configurations, the one of low deformation has a smooth onset, most prominent in the krypton and rubidium isotopic chains, and the one of large deformation has a sudden onset around $N=60$ (when it also becomes the more bound). The onset of oblate deformation gradually shifts towards $N=60$ as one approaches the zirconium chain, indicating the influence of the $Z=40$ proton sub-shell. This sub-shell effect seems to also influence the prolate configuration before $N=60$, however the emergence of the more deformed prolate minimum is less sensitive to its proximity.

The value of the pairing interaction does not qualitatively influence these general observations, but, as can be seen in Fig. 4.2, it has a significant quantitative impact. First of all, the spherical $S_{2 n}$ trends are lower for weaker pairing interaction. This can be understood by the fact that pairing correlations produce additional nuclear binding for the neutrons. Second of all, the deformation energy of the prolate and oblate configurations is larger for weaker pairing. This means that around the shape-transition point the overall $S_{2 n}$ evolution with the decrease of the pairing strength is the result of the competition between two effects: a lowering due to a generally less attractive interaction and an increase due to the enhancement of the deformation-energy slope with neutron number.

The effect of the pairing interaction on the relative mean-square charge radii is of a general increase with the reduction of the pairing strength. This is most clearly observed for the oblate configuration, as well as for the highly-deformed prolate configuration. The reason is that a reduction of the pairing interaction allows the mean-field to attain an equilibrium shape of larger deformation and hence larger root-mean-square radius. This is already an illustration of the competition between particle-particle and particle-hole correlations in the mean-field approach. The most obvious one visible in Fig. 4.2 is the location of the transition between the two prolate configurations. One observes that for a weaker pairing interaction $\left.\left(V_{0}=-150 \mathrm{MeV} \mathrm{fm}\right)^{3}\right)$ the transition takes place towards $N=58$, while for a stronger interaction $\left(V_{0}=-200 \mathrm{MeV} \mathrm{fm}^{3}\right)$ the border of the region of large deformation moves towards $N=60$.

The characteristics of each deformed configuration, but also the energy balance between them, are essential for defining the trends of the ground-state observables. Irrespective of the pairing strength, the calculations predict that before $N=60$ the oblate configuration is lower in energy than the spherical and prolate ones. After the transition of the prolate solution to a more deformed shape, the latter eventually becomes more bound, as was also shown in Fig. 4.1 for the krypton isotopes (with one exception). This 
transition between an oblate and a prolate ground-state shape determines similarly pronounced signatures in $\left\langle\delta r^{2}\right\rangle$, the only change with the pairing strength being the location of the transition point along the isotopic chain. For $S_{2 n}$, however, due to the way it influences the energy balance between the oblate and prolate configurations, the pairing strength is reflected in how pronounced the $S_{2 n}$ signature is at the transition point.

For a pairing strength $V_{0}=-200 \mathrm{MeV} \mathrm{fm}^{3}$, the prolate configuration describes remarkably well the experimental $S_{2 n}$ and $\left\langle\delta r^{2}\right\rangle$, both in magnitude and location of the shape transition. Considering however the ground-state in the mean-field approximation, one notices (especially in the charge radii) that the oblate-to-prolate transition is located at $N=61$ or beyond and that, as a result, the $S_{2 n}$ trend only slightly changes in slope. In the krypton isotopic chain, the ground-state configuration remains oblate at every neutron number (see also Fig. 4.1), although the prolate solution of higher deformation does emerge at $N=60$.

Lowering the strength of the pairing interaction to $V_{0}=-175 \mathrm{MeV} \mathrm{fm}^{3}$, the transition of the prolate configuration from low to high deformation shifts to lower neutron numbers by roughly one unit, such that the description of the experimental observables by the prolate configuration alone is less accurate. The description provided by the global minimum of all configurations, which approximates the ground state, is however improved. The oblate-to-prolate transition takes place around $N=60$ and the experimental $S_{2 n}$ and $\left\langle\delta r^{2}\right\rangle$ values are rather accurately described around the transition point by the lowestenergy configuration. In the krypton isotopic chain, the prolate configuration does become the ground-state at $N=62$, but the available experimental data, extending only up to $N=61$, do not exclude this possibility. The $S_{2 n}$ trends decrease even more with respect to the experimental values close to $N=50$.

Further reducing the pairing strength to $V_{0}=-150 \mathrm{MeV} \mathrm{fm}^{3}$ produces a general worsening of the agreement with experiment, both for the individual configurations and for the global minimum. The $\left\langle\delta r^{2}\right\rangle$ values of the more deformed prolate configuration are systematically larger and the $S_{2 n}$ values close to $N=50$ are significantly lower than the experimental trends. The transition from low to high deformation in the prolate minimum takes place roughly one neutron number earlier than for $V_{0}=-175 \mathrm{MeV} \mathrm{fm}{ }^{3}$. The position of the oblate-to-prolate transition does not change significantly, but the strong gain of oblate deformation energy already before $N=60$ makes the onset of oblate deformation, not the oblate-to-prolate transition, the main contribution to the $S_{2 n}$ kink close to $N=60$.

The experimental $S_{2 n}$ [Nai10] and $\left\langle\delta r^{2}\right\rangle$ [Kei95] of the krypton isotopes do not exhibit the sharp increase at $N=60$ which is observed for the other isotopic chains represented in Fig. 4.2. This lead to the interpretation of the krypton isotopic chain as the lower boundary of the quantum phase transition taking place in the neutron-rich $A \approx 100$ region of the nuclear chart [Nai10]. This conclusion contradicted the low energy tentatively proposed in [Mar09] for the first excited $2^{+}$state in ${ }^{96} \mathrm{Kr}(241 \mathrm{keV})$. The energy was 
later corrected to $554.1(5) \mathrm{keV}$ by a Coulomb excitation experiment on ${ }^{96} \mathrm{Kr}$, performed at ISOLDE [Alb12], suggesting, in agreement with the mass and charge-radii results, a smooth onset of quadrupole deformation.

The calculations presented in Fig. 4.2 show that an explanation of the different behavior of the krypton isotopes can already be provided at the level of the mean-field approximation. The predicted balance between the oblate and prolate configurations changes significantly between the rubidium and krypton isotopic chains. For a stronger pairing interaction $\left(V_{0}=-200 \mathrm{MeV} \mathrm{fm}{ }^{3}\right)$, the oblate-to-prolate transition takes place in the rubidium chain, but not in the krypton chain, as can be seen in Figs. 4.2(e) and 4.2(f). For a weaker pairing interaction $\left(V_{0}=-175,-150 \mathrm{MeV} \mathrm{fm}^{3}\right)$, the transition between the two configurations does take place in the krypton chain, but it is shifted to higher neutron number than the extension of the available experimental data. A persistence of the oblate configuration in the nuclear ground state or a later transition to the prolate configuration are possible reasons for the different properties of the krypton isotopes.

A study of the same nuclides using the HFB-SLy4 approach, but a spherical HO basis, was presented in [Man13], reaching similar conclusions. This reinforces the robustness of the presented results with respect to the choice of $\mathrm{HO}$ basis.

To understand what would change going beyond the static mean-field approximation, Fig. 4.3 shows theoretical two-neutron separation energies and mean-square charge radii of neutron-rich $A \approx 100$ nuclei obtained by two models from the literature which use beyondmean-field techniques: the generator-coordinate-method approach of [Ben06], using the BCS approximation and the SLy4 interaction and the mapped-collective-Hamiltonian approach of [Del10], based on the HFB approximation and the Gogny-D1S interaction. As the calculations are only available for even-even nuclei, only the experimental data of even- $Z$ isotopic chain are presented for comparison. For each of the two approaches, the results are presented for the static mean-field minimum (generically named "static") and for the beyond-mean-field result (named "dynamic").

Concerning the two-neutron separation energies, Fig. 4.3(a) shows that for both models the biggest difference brought on by the inclusion of dynamic correlations (at the beyondmean-field level) is close to $N=50$. Only considering static deformation, the $S_{2 n}$ values fall significantly below the experimental ones at $N=52$, which is also observed for the calculations presented in Fig. 4.2. Dynamic correlations lead to an increase of $S_{2 n}$ in this region, bringing it much closer to the experimental values. In the region of rigid deformation, however $(N \geq 60)$, not much changes once shape fluctuations are taken into account. One notices also that the predicted $S_{2 n}$ trend close to the shape-transition point is very straight and that the experimental values of the well-deformed nuclei are systematically underestimated.

Concerning the charge radii, one notices the generally smoother trend obtained going beyond the mean-field level. It is important to note that (with few exceptions) all charge radii are predicted to be larger in absolute value once dynamic correlations (shape fluc- 
tuations) are allowed. This is also true for the reference, $N=50$ nuclei, which means that a decrease of $\left\langle\delta r^{2}\right\rangle$ is in most cases a reflection of the fact that the charge radius of the reference isotope increased more significantly than the one of the isotope in question. This is the case of the strontium isotopes with $N \geq 60$, which shows that, especially when the reference isotope is spherical, an overestimation of $\left\langle\delta r^{2}\right\rangle$ at the static mean-field level can also be related to an underestimation of the radius of the reference isotope, in the absence of any dynamic contribution.

One observes however also the reverse effect for the zirconium isotopic chain. Especially in the D1S calculation, the charge radii show no signs of an onset of deformation at the static mean-field level, which however is clearly visible once dynamic correlations are taken into account. The zirconium nuclei are thus not predicted to have a strong static deformation, but to be very "soft", the dynamic contribution to the charge radius increasing with the addition of neutrons.

A fact which cannot be observed in Fig. 4.3(b) is that the charge radii (not only $\left\langle\delta r^{2}\right\rangle$ ) of ${ }^{96,98} \mathrm{Kr}$ are predicted by the D1S calculation to decrease once collective fluctuations are allowed. This means that, for explaining the different behavior of krypton isotopes around $N \approx 60$, the answer might also reside in the effect of collective motion on the nuclear shape.

Experimental information on the sign of the intrinsic quadrupole moment can be obtained by laser spectroscopy, however this information is scarce for the neutron-rich $A \approx 100$ nuclides. The $N=51$ isotones with $Z=36-40$ are slightly oblate [Kei95; Thi81; Buc90; Che07; Tha03]. The existence of slightly oblate shapes for $N<60$ and pronounced prolate shapes for $N \geq 60$ in the yttrium isotopic chain is shown in [Che07], however measurements for $N<60$ mostly refer to low-lying isomeric states (exception being $N=51,55)$. The rubidium isotopes with $N=52,54,56-59$ are however determined to be slightly prolate [Thi81], as well as the krypton isotopes with $N=53,55$ [Kei95].

In Fig. 4.2 one notices that the strength of the pairing interaction also affects the predicted sharpness of the $S_{2 n}$ drop at the crossing of $N=50$. Since this is the twoneutron shell gap $\Delta_{2 n}$ defined in Eq. (1.18), it is interesting to study how the pairing strength affects the description of this very important quantity, which is regarded as a reference observable for testing the predictions of effective nuclear interactions [Sor08]. The theoretical values of the $N=50$ two-neutron shell gap obtained for different strengths of the pairing interaction are presented in Fig. 4.4(a). The experimental values obtained with masses from AME2012 [Wan12], completed by the recent ISOLTRAP measurement of the mass of ${ }^{82} \mathrm{Zn}$ [Wol13a], are presented for comparison. Figure 4.4(b) shows the results of the self-consistent mean-field calculations of [Ben06; Ben08] at two levels of approximation (similarly to Fig. 4.3): for the static mean-field minimum (full blue circles, labeled "static") and for the solution including dynamic correlations (empty blue circles, labeled "dynamic").

The trend of the two-neutron shell gap is the sum of a number of effects (see [Ben08] 


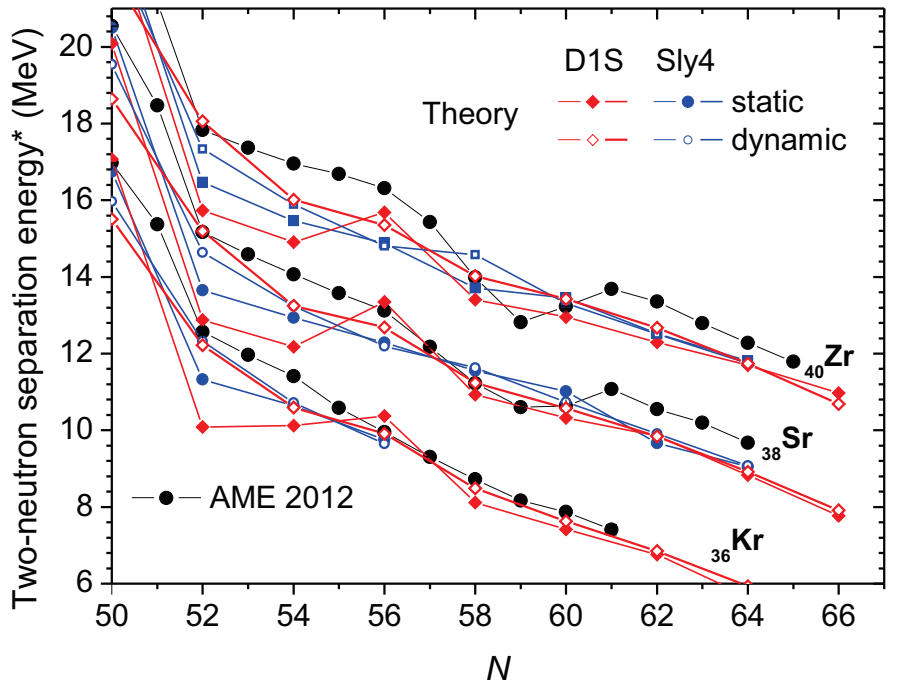

(a)

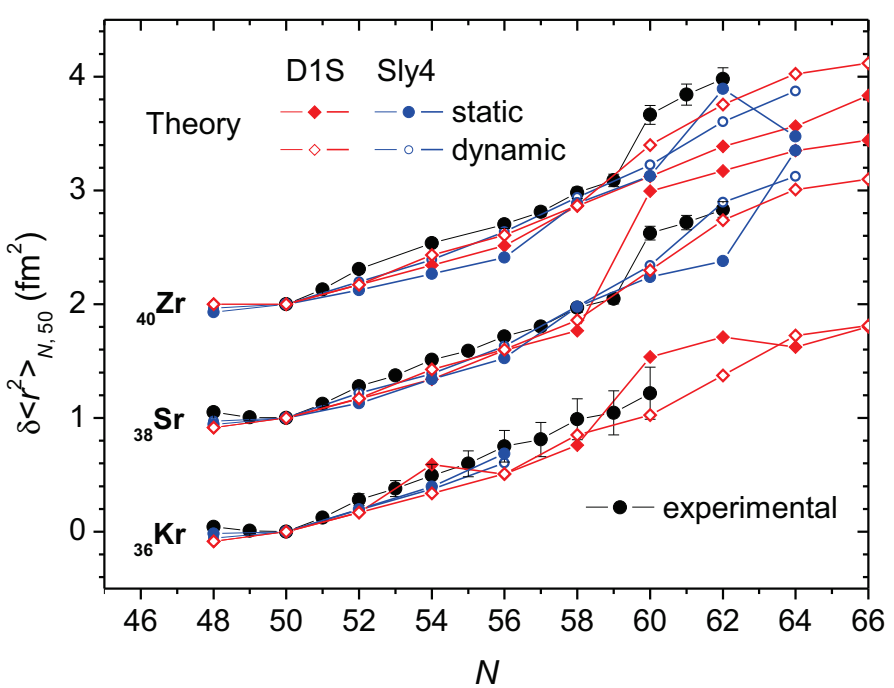

(b)

Figure 4.3: Experimental two-neutron separation energies and mean-square charge radii of even$Z$, neutron-rich $A \approx 100$ nuclei, compared to self-consistent mean-field calculations from the literature. The experimental $S_{2 n}$ values use masses from the AME2012 [Wan12]. The $\left\langle\delta r^{2}\right\rangle$ values are taken from [Kei95; Buc90; Lie91; Cam97; Cam02; Tha03]. The theoretical values are obtained for even-even nuclei in the HFB-D1S (Gogny) [Del10] or BCS-SLy4 [Ben06] framework (red diamonds and blue circles, respectively). In both cases, the figure shows the static meanfield solutions (full symbols) and beyond-mean-field results, which include dynamic correlations (empty symbols). Except for the krypton chain, the $S_{2 n}$ and $\left\langle\delta r^{2}\right\rangle$ values are biased for better separation. 


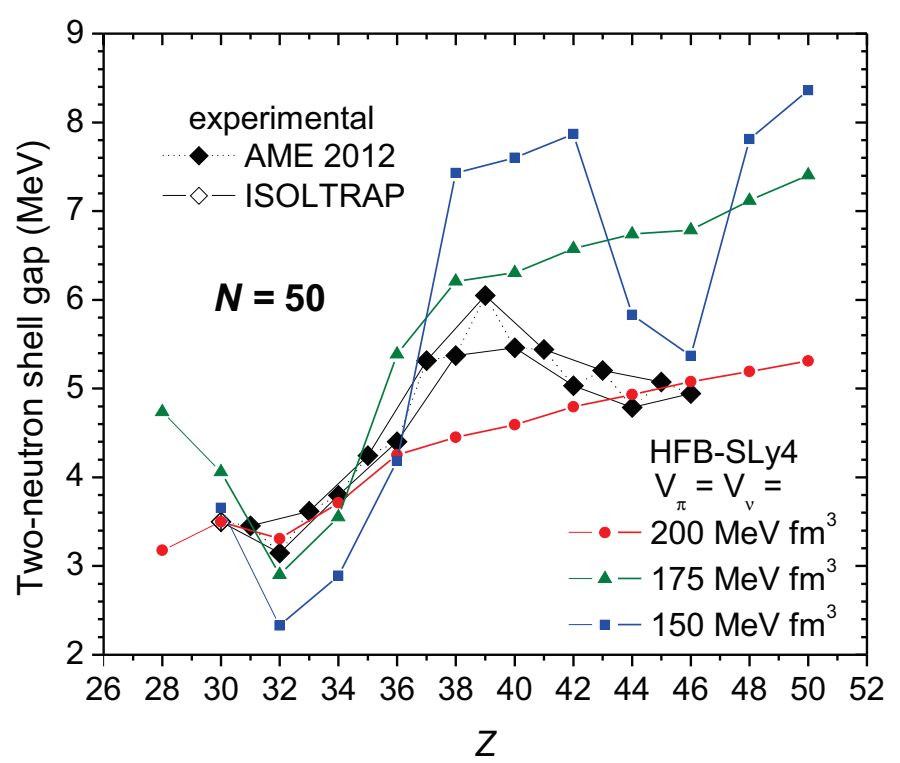

(a)

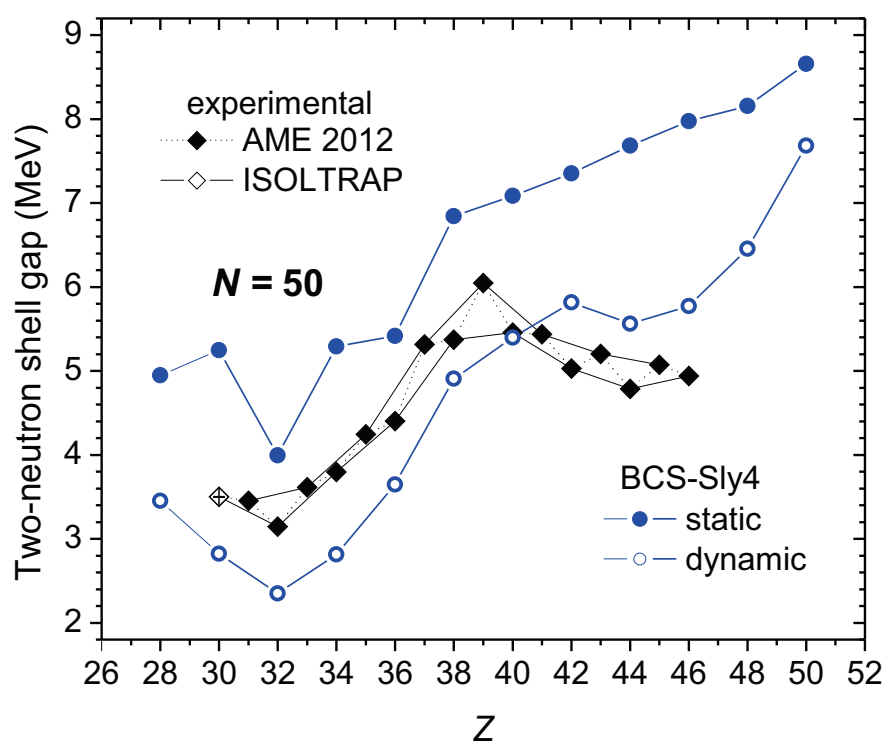

(b)

Figure 4.4: Theoretical values of the $N=50$ two-neutron shell gap compared to experimental values obtained using masses from AME2012 [Wan12] and the recent ISOLTRAP measurement of the mass of ${ }^{82} \mathrm{Zn}$ [Wol13a]: (a) shows HFB-SLy4 results obtained in the present work using the static mean-field approximation, for different strengths of the pairing interaction; (b) shows literature BCS-SLy4 results [Ben06] obtained at the static mean-field level (full circles) and including dynamic correlations through the generator coordinate method (empty circles). 
and Chapter 1). The zero-order contribution is the energy gap between the delimiting single-particle levels (the "real" shell gap). However, because the definition of $\Delta_{2 n}$ involves the properties of open-shell nuclei, in which pairing and quadrupole correlations are active, higher-order contributions from the derivatives of the correlation energy distort the trend of $\Delta_{2 n}$ with respect to the gap in the single-particle spectrum. For nuclides close to magic numbers, quadrupole correlations have a static contribution, which is described by the deformation of the nuclear mean-field, and a dynamic contribution, which is related to zero-point collective motion and is described at the beyond-mean-field level [Ben08]. The quadrupole correlation energy of open $N$-shell nuclei is larger than for the magic- $N$ isotones. It is thus easy to show that the static and dynamic correlations have the effect of lowering the value of the two-neutron shell gap. Since the calculations presented here only account for static correlations through the deformation of the mean-field, the results of Fig. 4.4(a) are only over-estimations of the theoretical $\Delta_{2 n}$. This is clearly illustrated in Fig. 4.4(b), where both the mean-field (static) and the "configuration-mixed" (dynamic) solutions are shown, the latter being systematically lower. At the same time, as for the HFB-SLy4 calculations of Fig. 4.4(a), the static solution of Fig. 4.4(b) only captures the $\Delta_{2 n}$ trend for $Z<40$, the addition of dynamic correlations being necessary for describing the entire evolution of $\Delta_{2 n}$ (especially for $Z>40$ ).

One notices in Fig. 4.4(a) that the two-neutron shell gap obtained for a value of the pairing interaction $V_{0}=-200 \mathrm{MeV} \mathrm{fm}^{3}$ underestimates the experimental values. Dynamic correlations would further reduce the $\Delta_{2 n}$ values of the open proton-shell nuclei. On the other side, a pairing strength $V_{0}=-150 \mathrm{MeV} \mathrm{fm}^{3}$ qualitatively reproduces the variations of $\Delta_{2 n}$, but overestimates their strength. The decrease of $\Delta_{2 n}$ around $Z=32$ is exaggerated. Finally, a pairing strength $V_{0}=-175 \mathrm{MeV} \mathrm{fm}^{3}$ reproduces the $\Delta_{2 n}$ trend for $32 \leq Z \leq 40$ and leaves room for a further decrease with the effect of configuration mixing. Considering also this subsequent step, a value $V_{0}$ close to $175 \mathrm{MeV} \mathrm{fm}^{3}$ seems optimal for the description of $\Delta_{2 n}$.

\section{$4.2 \quad$ Neutron-deficient gold-thallium nuclei}

Nuclear structure of the neutron-deficient isotopes with $Z \leq 82$ rapidly evolves from essentially spherical configurations in the ground states of the lead isotopes $(Z=82)$ [Wit07], with coexisting deformed configurations in the excitation spectrum [And00], to static deformation in the ground states of mid-shell gold nuclei $(Z=79)$, following a shape transition form low to large deformation at $N=107$. As in the case of the n-rich $A \approx 100$ isotopes, the known gold charge radii describe the ground-state picture most clearly [Wal87; Wal89; Sav90; Bla97]. The thallium $(Z=81)$ and mercury $(Z=80)$ isotopes exhibit intermediate properties. The known data in the thallium isotopic chain show no signs of a shape transition, with the notable existence of a low-lying $I=9 / 2$ isomer in the even- $N$ thallium isotopes, interpreted as the excitation of a proton across 
the $Z=82$ shell gap to the $h_{9 / 2}$ orbital. The excitation energy of this isomer reaches a minimum close to the neutron mid-shell [Aud12], meaning that thallium better matches the shape-coexistence picture of the neighboring lead isotopes, with close to spherical ground states and deformed configurations at higher energy (see [Bar13] for a recent update on the measured charge radii). The mercury isotopes, however, exhibit a shape transition at $N=105$ [Bon72; Bon76], with a strong odd-even staggering of the charge radii [Kuh77; Ulm86], suggesting the proximity in energy of nuclear configurations with significantly different shapes. This behavior is a precursor of what is observed in the gold chain and is supported by recent data from the spectroscopy of even-even mercury isotopes [Bre14].

With this complex nuclear landscape at hand, one is interested in how the differential mass quantities reflect the rapidly changing nuclear structure with the removal of protons from the $Z=82$ core. As was observed for the neutron-rich $A \approx 100$ nuclei, the study of mean-field configurations and their energy balance can provide the explanation for the observed systematics of ground-state properties in shape-transition regions. Consequently, the HFB-SLy4 calculations with a contact pairing interaction of volume type Eq. (3.25) were also applied to the study of the neutron-deficient gold, mercury and thallium isotopes. Only the quadrupole degree of freedom was studied, as it gives the leading-order contribution to the nuclear deformation of the studied nuclides. In a first stage, the calculations were performed only for the HFB quasi-particle vacuum, without any blocking prescription. Different values of the pairing interaction were analyzed. This allowed extending the study of the competition between particle-particle and particle-hole correlations to a second shape-transition region of the nuclear chart.

As in the case of the neutron-rich $A \approx 100$ nuclei, the neutron-deficient gold-thallium isotopes exhibit both oblate and prolate equilibrium configurations of the nuclear meanfield. Figure 4.5 shows the deformation energy of these configurations along the gold isotopic chain, for different values of the pairing strength. For nuclei exhibiting the coexistence of several oblate or prolate minima, the one lowest in energy is represented in Fig. 4.5 for each type of configuration. One notices, as for Fig. 4.1, the competition in energy between the oblate and the prolate configurations. Following the isotopic chain from $N=120$ downward, the oblate configuration is initially more bound, but at $N=105$ the ordering in energy between the two configurations changes. Again, lowering the strength of the pairing interaction increases the deformation energy of both configurations, but it affects them differently. The prolate configuration gains more deformation energy than the oblate one, for the same change in pairing strength, which is most clearly observed for $N<105$.

The predicted evolution of the trends of nuclear observables in the ground states of the studied isotopes is a direct reflection of this competition. The two-neutron separation energies and mean-square charge radii of the gold, mercury and thallium isotopes are compared in Fig. 4.6 to the available experimental data. The experimental two-neutron 


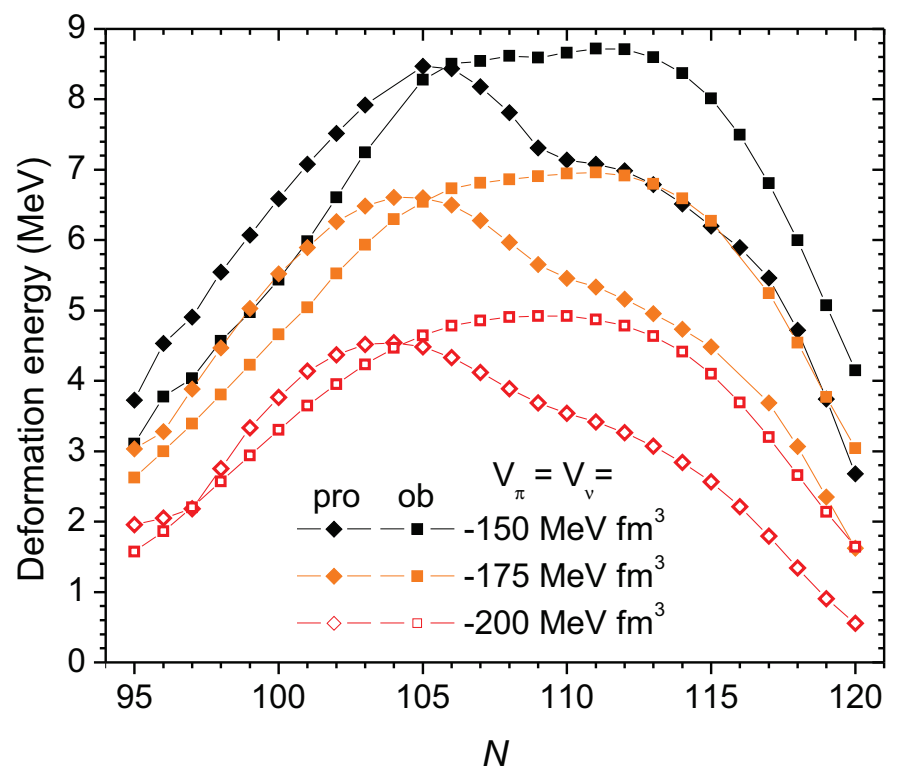

Figure 4.5: Deformation energy of the prolate and oblate equilibrium configurations (diamonds and squares, respectively) of the gold isotopes, obtained at the level of the HFB quasi-particle vacuum, using different values of the pairing interaction. For each isotope, the minimum-energy prolate and oblate configuration is represented.

separation energies use masses from the AME2012 [Wan12] and the masses of gold isotopes which were analyzed as part of the present work. Some of the thallium masses used for Fig. 4.6 are part of a recently-published set of ISOLTRAP measurements, already included in AME2012 [Boh14], where the shape of thallium isotopes is discussed. The experimental mean-square charge radii are the evaluated values from [Fri04a; Fri04b] and [Bar13]. They are represented, as in the case of Fig. 4.2, by the displacement with respect to a reference isotope. Except for the gold isotopes, both experimental and theoretical values are shifted upward by the same amount for better separation. The experimental uncertainties of the $\left\langle\delta r^{2}\right\rangle$ values are smaller than the used symbols and are not shown. Systematic uncertainties which result from the analysis of the isotope-shift data for determining the charge radii are not represented. They can affect the slope of the $\left\langle\delta r^{2}\right\rangle$ trends with neutron number.

The calculations are initially performed for the same three values of the pairinginteraction strength used for the neutron-rich $A \approx 100$ nuclei, namely $V_{0}=-150,-175$, and $-200 \mathrm{MeV} \mathrm{fm}^{3}$. The same strength was employed both for the proton and for the neutron pairing. The calculations are presented separately for the minimum-energy oblate and prolate configuration. The global minimum of all configurations, to which the ground state is assigned, is marked distinctively. For the theoretical mean-square charge radii, the 
reference value is the one corresponding to the global minimum of the $N=115$ isotope. Similarly to the $A \approx 100$ nuclei, a few general observations can be made irrespective of the value of the pairing interaction. The gold-thallium nuclides exhibit oblate and prolate minima of the self-consistent mean field along the entire computed range, $95<N<120$, which are more bound and of larger charge radius than the corresponding spherical configuration. The oblate configuration has a smooth evolution with neutron number, which is reflected in the $S_{2 n}$ and $\left\langle\delta r^{2}\right\rangle$ trends. Decreasing the neutron number from $N=120$, the charge radii gradually depart from the spherical trend towards mid-shell $(N=104)$ and re-approach it for $N<104$. This smooth behavior can also be observed in the deformation energy of the oblate solutions represented in Fig. 4.5.

The prolate configuration has a similarly smooth trend towards $N=120$, determined by an energy minimum situated at low quadrupole deformation. Removing neutrons along the isotopic chain, a transition eventually occurs to a large-deformation prolate minimum, which remains the dominant (prolate) configuration until, for a certain neutron number $N<100$, a transition back to the low-deformation minimum occurs. These transitions are accompanied by large changes in the mean-square charge radius and in the trend of the two-neutron separation energies for the prolate configuration. The cause of the peculiar behavior of $S_{2 n}$ is best explained by in the evolution of the deformation energy of the prolate solution represented in Fig. 4.5. One notices that, with the emergence of the prolate minimum at large deformation, the slope of the deformation energy with neutron number changes significantly. Having in mind Eq. (4.3), this change of slope determines the large kink in $S_{2 n}$ close to the transition point between the two prolate minima.

The oblate minimum is invariably the one lowest in energy towards $N=120$ for any of the three studied isotopic chains, giving the ground-state configuration ("global minimum" in Fig. 4.6). A few neutrons less than the emergence of the large-deformation prolate solution, the latter becomes more bound than the oblate solution and the groundstate changes from oblate to prolate. The ground-state charge radii suddenly increase by the removal of a single neutron. Towards $N=95$, a sudden reduction of the ground-state charge radii is predicted once the prolate configuration changes back to low deformation. With this change, the ground-state deformation is predicted in some cases to remain prolate (e.g. all studied chains for $V_{0}=-150 \mathrm{MeV} \mathrm{fm}^{3}$ ) and in others to return to oblate (e.g. all studied chains for $V_{0}=-200 \mathrm{MeV} \mathrm{fm}^{3}$ ).

Because the difference in energy between the oblate and prolate configurations varies smoothly around the transition between them, the ground-state $S_{2 n}$ values do not exhibit very sharp signatures indicating the occurrence of the shape transition. Instead of the sudden $S_{2 n}$ kink observed for the prolate configuration, the ground state only shows a flattening of the $S_{2 n}$ trend around the transition point.

Beyond these general qualitative statements, the strength of the pairing interaction has some obvious quantitative effects on the two-neutron separation energies and charge radii. As for the neutron-rich $A \approx 100$ nuclei, weaker pairing corresponds to generally 


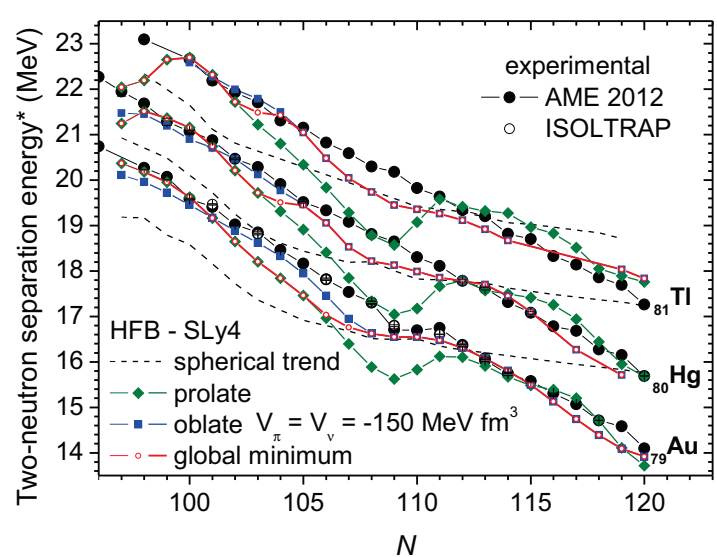

(a)

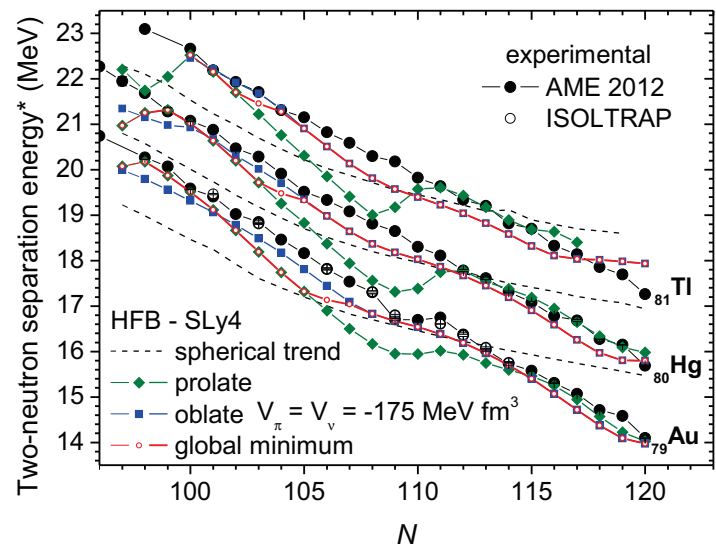

(c)

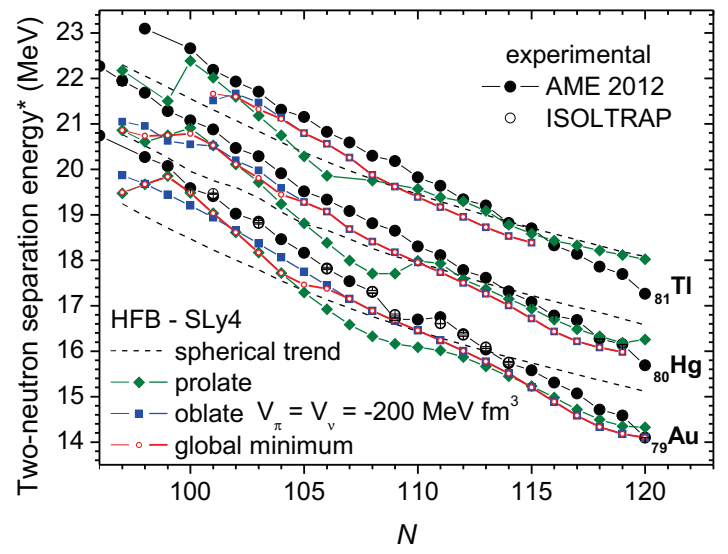

(e)

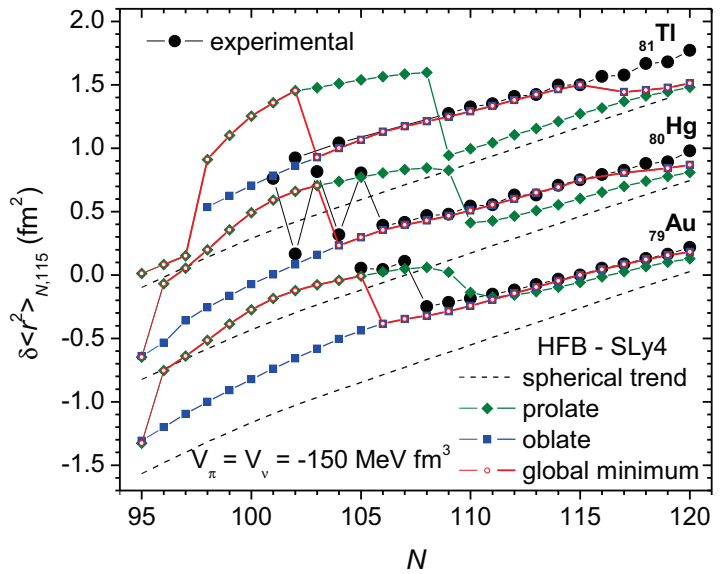

(b)

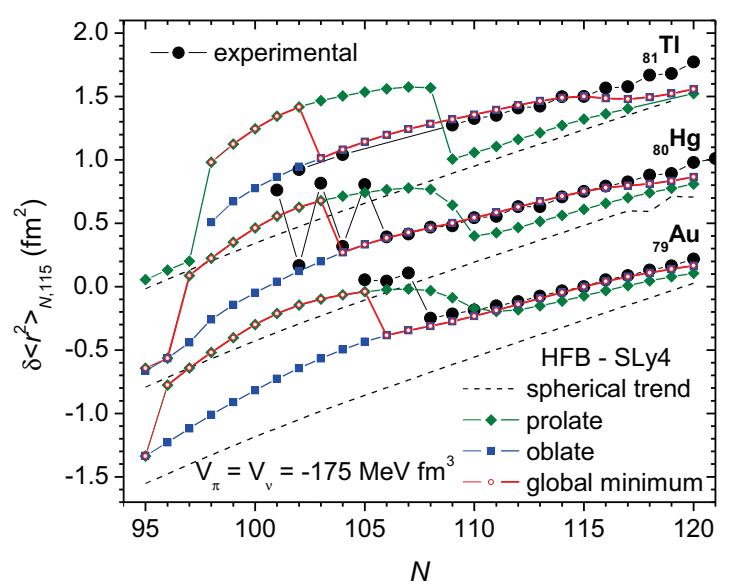

(d)

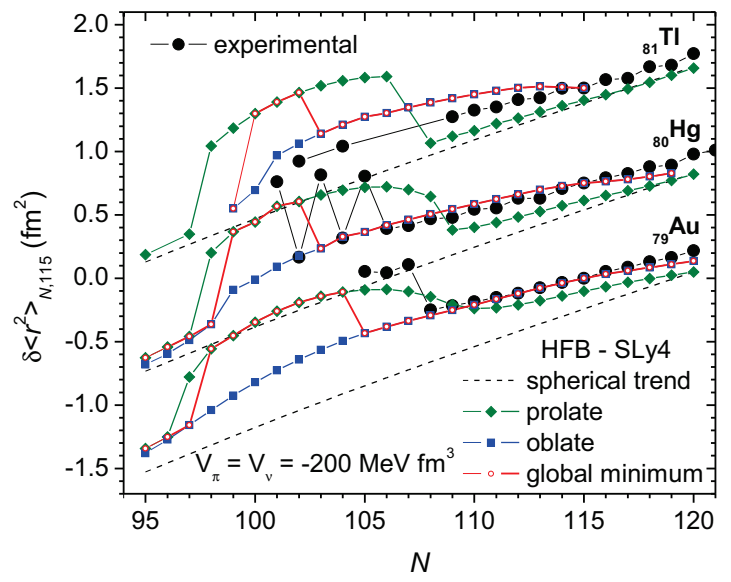

(f)

Figure 4.6: Experimental two-neutron separation energies and mean-square charge radii of neutron-deficient gold-thallium nuclei, compared to HFB-SLy4 calculations. The experimental $S_{2 n}$ values use masses from the AME2012 [Wan12] and the present work. The $\left\langle\delta r^{2}\right\rangle$ values are taken from [Fri04a; Fri04b] and [Bar13]. The theoretical values represent the HFB quasiparticle vacuum (no blocking) of the spherical, lowest-energy oblate and lowest-energy prolate configurations (dashed line, blue squares and green diamonds, respectively). The red empty circles represent the values corresponding to the ground-state, interpreted as the configuration of lowest energy. Except for the gold chain, the $S_{2 n}$ and $\left\langle\delta r^{2}\right\rangle$ values are biased for better separation. 
larger charge radii. The deformation energy gained by reducing the pairing strength overcomes the overall reduction of the force's attractiveness, meaning that weaker pairing also corresponds to larger $S_{2 n}$ values. A pairing strength $V_{0}=-200 \mathrm{MeV} \mathrm{fm}{ }^{3}$ leads to systematically too low theoretical values of $S_{2 n}$, while a value of $V_{0}=-150 \mathrm{MeV} \mathrm{fm}{ }^{3}$ leads to excellent agreement with experiment towards the beginning and the end of the neutron shell.

The pairing interaction influences the $S_{2 n}$ trend for each equilibrium configuration. Weaker pairing increases the strength of the $S_{2 n}$ kink for the prolate configuration around the borders of the region of high deformation and also enhances the flattening of $S_{2 n}$ for the oblate configuration around mid-shell. This in the end affects the $S_{2 n}$ trend of the global minimum and increases the region of $S_{2 n}$ flattening for the oblate-to-prolate transition in the ground state of the gold isotopes (see Fig. 4.6(a)).

A very important effect of the pairing interaction is still related to the extension along the isotopic chain of the large-deformation prolate minimum and to the location of the ground-state transition from oblate to prolate deformation. This effect is, as for the neutron-rich $A \approx 100$ nuclei, a direct illustration of the competition between particleparticle and particle-hole correlations in nuclei. With the reduction of the pairing strength, the number of isotopes in the region of large deformation increases for the prolate configuration and for the global minimum. The effect is most pronounced on the low- $N$ side of the region of large deformation. Although an effect of the pairing strength is observed on the high- $N$ side too, the oblate-to-prolate transition (while removing neutrons along the isotopic chain) is predicted to take place two or three neutron numbers later than observed in experiment for the gold and mercury isotopes, even when using a pairing strength $V_{0}=-150 \mathrm{MeV} \mathrm{fm}^{3}$.

One notes that the return to a less deformed ground-state configuration for $N<100$ produces a significant kink in $S_{2 n}$ which is not observed in the available experimental data. This can be interpreted either as a sign of the transition to low deformation taking place for lower neutron number than the extension of the calculations or its steepness being less pronounced than predicted. We note that a lower pairing interaction brings an improvement from this point of view.

The oblate-to-prolate transition is predicted to also take place in the ground state of the thallium isotopes, for any of the studied pairing-interaction strengths. One observes in Fig. 4.6 that the comparison of theoretical and experimental $S_{2 n}$ values cannot rule out this transition taking place, due to the smoothness of the calculated $S_{2 n}$ trend, while the available thallium charge-radii data do not extend far enough for the comparison to be possible. The known complementary experimental data suggest however that the ground states of the thallium isotopes remain almost spherical across the neutron mid-shell. This disagreeing with the picture of Fig. 4.6, one notes that the mean-field description of isotopes so close to proton magicity $(Z=82)$ as the thallium isotopes might no longer be accurate, because of missing shape fluctuations which can only be described beyond 
the static mean-field picture [Ben06]. A significant worsening of the agreement with experiment is also observed for mercury and gold isotopes with $N>115$, which can also be attributed to the unsuitability of the static mean-field approximation for nuclei so close to the neutron magic number $N=126$. A similar phenomenon is observed close to $N=50$ for the neutron-rich $A \approx 100$ nuclei.

As in the case of the neutron-rich $A \approx 100$ nuclei, one is interested in the way the twoneutron separation energies and mean-square charge radii are affected by the addition of dynamic correlations, going beyond the mean-field approximation. Referring to the same two theoretical approaches illustrated in Fig. 4.3 [Ben06; Del10], Fig. 4.7 presents the $S_{2 n}$ and $\left\langle\delta r^{2}\right\rangle$ values obtained for the even-even mercury isotopes at the static mean-field level (labeled "static") and including dynamic correlations though configuration mixing (labeled "dynamic"). Experimental values are shown for comparison. In what concerns the two-neutron separation energies, as the HFB-SLy4 calculations presented in Fig. 4.6, the literature results systematically underestimate the experimental values. Noteworthy is that the mean-field results also predict a sharp kink in $S_{2 n}$ close to $N=95$ and a

flattening of the $S_{2 n}$ slope around $N=105$. However, the $S_{2 n}$ signatures disappear once dynamic correlations are taken into account, bringing the theoretical results in close agreement to experiment.

The main features of the mean-square charge radii are not changed for the ground states of low deformation, once dynamic correlations are taken into account. This is not unexpected, since their experimental $\left\langle\delta r^{2}\right\rangle$ trends are quite well described by the HFBSLy4 calculations in the mean-field approximation (unlike the low-deformation groundstates of the neutron-rich $A \approx 100$ nuclei). Still, the predicted borders of the region of large deformation are slightly different at the beyond-mean-field level with respect to the static picture. One notes that the mean-field results of both approaches presented in Fig. 4.7 predict the ground-state shape transition at $N \approx 105$ (decreasing the neutron number) to be from oblate to large-prolate deformation. The average deformation of the solution obtained after configuration mixing is predicted in [Ben06] to preserve this sequence.

\subsection{Odd-even staggering of mercury isotopes}

In order to test the strength of the pairing interaction also against the description of the odd-even staggering, the odd mercury isotopes were computed in the self-consistent blocking approximation. For each odd isotope, eleven candidate single-particle states were tested for choosing the optimal one-quasi-particle state (by the overlap method [Dob09b; Sch10]). The stability of the obtained self-consistent solutions was worse than for the fully-paired calculations, the algorithm suffering from the same type of stability problems as the ones reported in [Sch10]. The mean stability of the results was better than $100 \mathrm{keV}$, enough to clearly trace the variation of the calculated odd-even staggering 


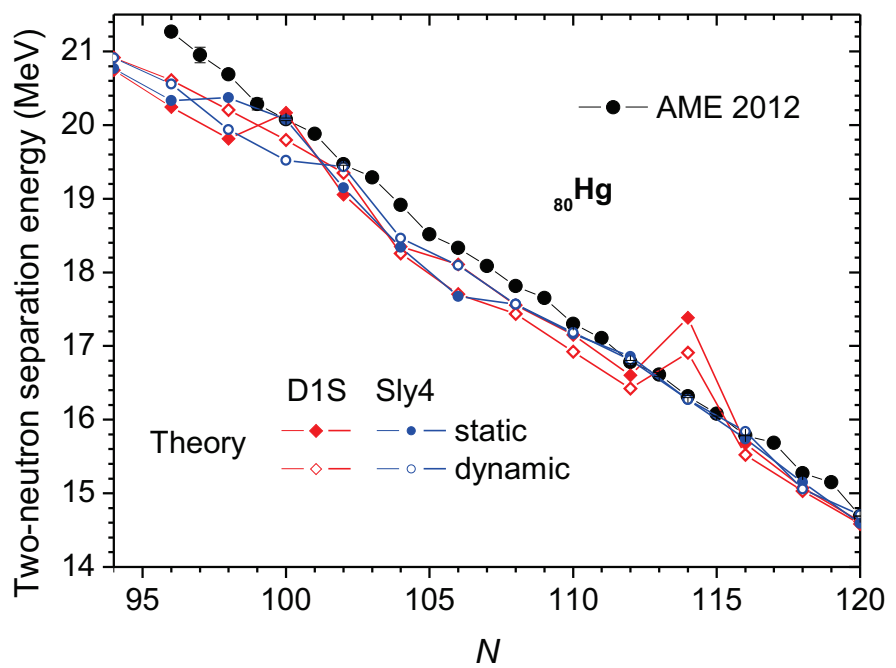

(a)

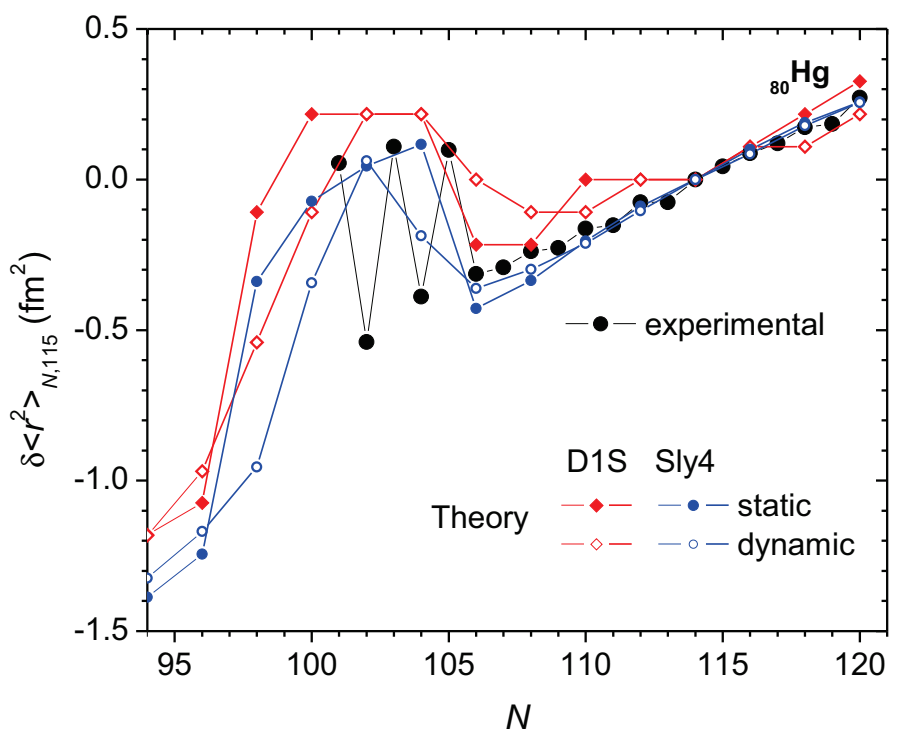

(b)

Figure 4.7: Experimental two-neutron separation energies and mean-square charge radii of neutron-deficient mercury isotopes, compared to self-consistent mean-field calculations from the literature. The experimental $S_{2 n}$ values use masses from the AME2012 [Wan12]. The $\left\langle\delta r^{2}\right\rangle$ values are taken from [Fri04b]. The theoretical values are obtained in the HFB-D1S (Gogny) [Del10] or BCS-SLy4 [Ben06] framework (red diamonds and blue circles, respectively). In both cases, the figure shows the static mean-field solutions (full symbols) and also the beyond-mean-field results, which include dynamic correlations (empty symbols). 
with the strength of the pairing interaction.

Figure 4.8 compares the calculated and experimental values of the five-point estimator of the odd-even staggering $\left(\Delta_{5 n}\right)$ along the mercury isotopic chain (also known as fivepoint empirical pairing gap), defined in Eq. (1.26). The experimental values use masses from AME2012 [Wan12]. The experimental values of $\Delta_{5 n}$ for the gold isotopes are also shown. The values obtained using the masses of gold isotopes analyzed as part of the present work are highlighted. They are systematically lower than the $\Delta_{5 n}$ values of the mercury isotopes, because the gold isotopes have odd proton number (see also Eq. (4.1)). Thus, the calculated $\Delta_{5 n}$ are not meant to be compared in absolute value to the experimental values of the gold isotopes. However, the relative trend of $\Delta_{5 n}$ along the gold isotopic chain can be used to interpret how nuclear deformation impacts the odd-even staggering of binding energies.

The calculations are presented for two different choices of the pairing-interaction strength, namely $V_{0}=-200$ and $-225 \mathrm{MeV} \mathrm{fm}^{3}$. For each choice, $\Delta_{5 n}$ is calculated separately for the minimum-energy prolate and oblate configuration, as well as for the global minimum of the self-consistent mean field. For an easier comparison, Fig. 4.9 presents only the $\Delta_{5 n}$ values corresponding to the global energy minimum (ground state) for the investigated strengths of the pairing interaction.

Figure 4.9 shows that the variation of the strength of the pairing interaction significantly influences the predicted magnitude of the odd-even staggering (only global minima shown). A value of the pairing strength $V_{0}=-200 \mathrm{MeV} \mathrm{fm}^{3}$ leads to a underestimation of the experimental "pairing gap". A volume pairing strength $V_{0}=-225 \mathrm{MeV} \mathrm{fm}{ }^{3}$ comes closer to the experimental $\Delta_{5 n}$, although it slightly overestimates it. A value of the pairing interaction between the two is necessary to reproduce on average the experimental data.

The calculated $\Delta_{5 n}$ values corresponding to the spherical configuration are presented in Figs. 4.8(a) and 4.8(b). One notices that in the spherical approximation the odd-even staggering is in general larger than the one obtained once deformation is taken into account. A correct prediction of the odd-even staggering of deformed nuclei thus requires not only a correct description of pairing correlations, but also of the quadrupole collectivity of the studied nuclei (as the dominant mode of nuclear collectivity). This becomes increasingly difficult for nuclei which are not statically deformed, but have a pronounced vibrational character, because the deformation of the nuclear mean field does not capture the full magnitude of the ground-state correlations. This is why the pairing interaction is typically constrained on the odd-even staggering of semi-magic nuclei [Ben03].

One notices for the different plots of Fig. 4.8 that the theoretical $\Delta_{5 n}$ values of the oblate and prolate configuration can differ, for the same pairing strength, by as much as a few hundred $\mathrm{keV}$. This means that, especially in shape-transition regions where the different mean-field minima come very close in energy, the final balance between the competing equilibrium configurations can only be decided once the blocking phenomenon is fully accounted for in the odd- $Z$ and odd- $N$ nuclei. When analyzing observables along 


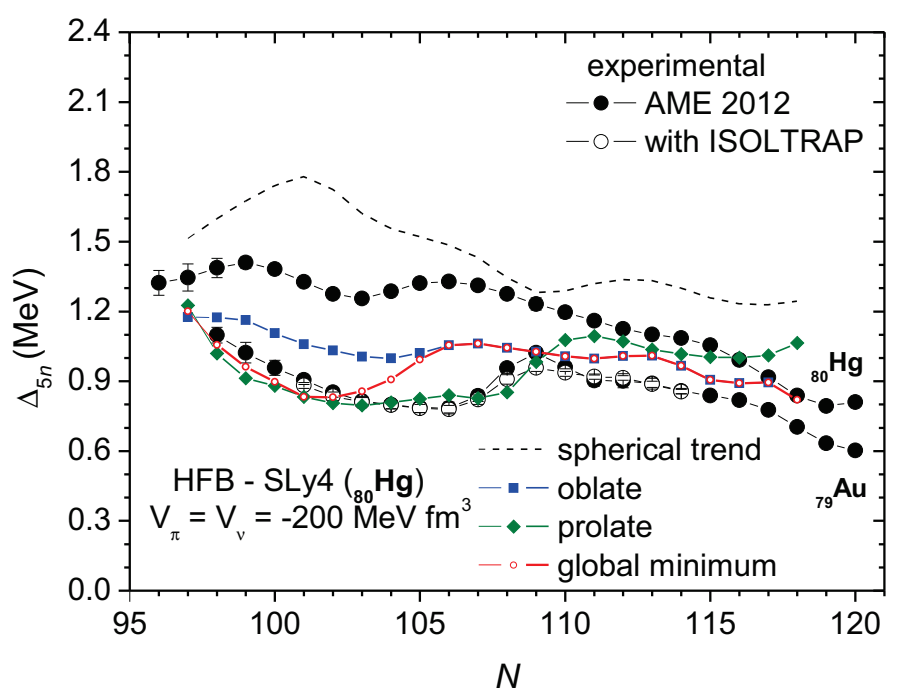

(a)

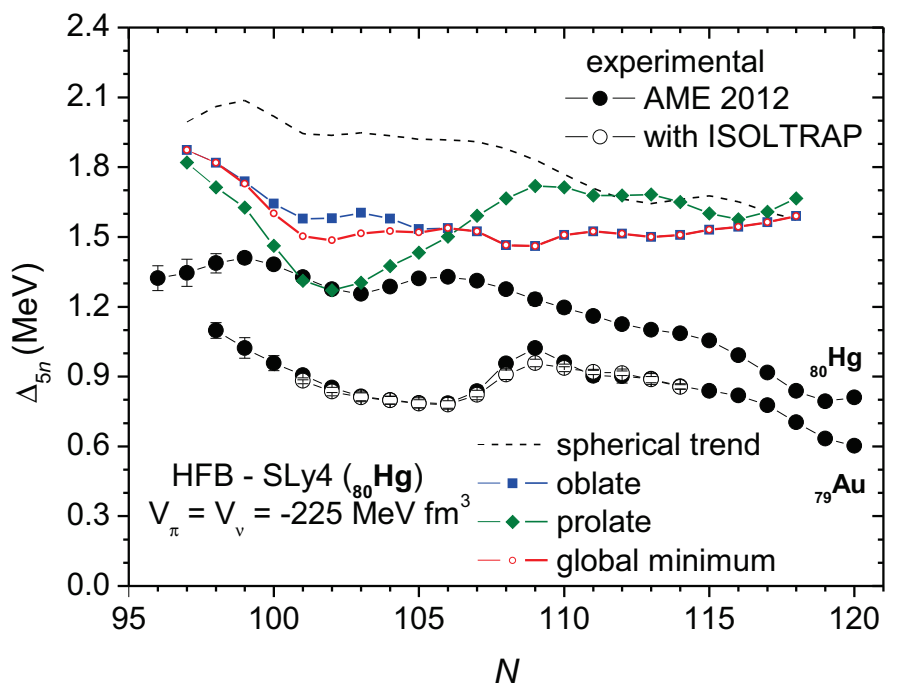

(b)

Figure 4.8: Experimental values of the five-point empirical pairing gap $\Delta_{5 n}$ (for gold and mercury isotopes) compared to the results of HFB-SLy4 calculations (for mercury isotopes). The experimental $\Delta_{5 n}$ values use masses from the AME2012 [Wan12] and the present work. The theoretical values are presented for two different strengths of the pairing interaction, $V_{0}=-200$ and $-225 \mathrm{MeV} \mathrm{fm}^{3}$. The results for the minimum-energy oblate and prolate configurations are each presented (full blue squares and full green diamonds, respectively), as well as the one corresponding to the global minimum, to which the nuclear ground state is assigned (red open circles). The trend of $\Delta_{5 n}$ for a calculation in spherical symmetry is also presented (dashed line). 


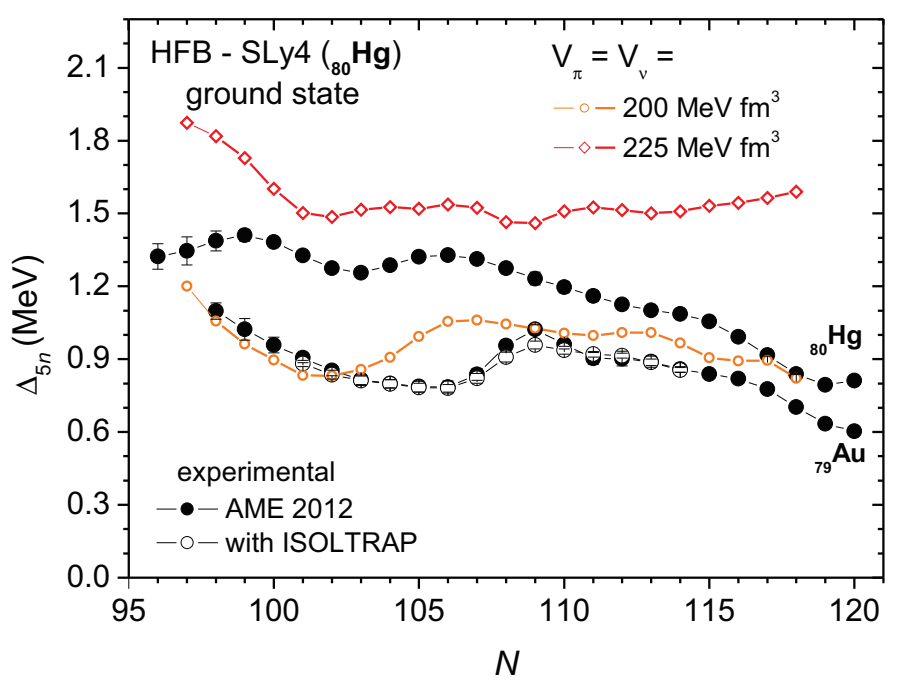

Figure 4.9: Experimental values of the five-point empirical pairing gap $\Delta_{5 n}$ for mercury isotopes compared to the results of HFB-SLy4 calculations. The experimental $\Delta_{5 n}$ values use masses from the AME2012 [Wan12]. The theoretical values correspond to the global mean-field minimum and are presented for two different strengths of the pairing interaction, $V_{0}=-200$ and $-225 \mathrm{MeV} \mathrm{fm}^{3}$.

isotopic chains of odd proton number, this requirement is valid for every isotope. The zeroquasi-particle solutions in odd and odd-odd systems give a good first-order description of the global trends of the nuclear observables, including their variation with the parameters of the energy functional. To decide on fine local effects, a full description of odd nuclei is however required.

Figures 4.10 and 4.11 show for comparison the results of calculations performed with and without quasi-particle blocking for pairing strengths $V_{0}=-200 \mathrm{MeV} \mathrm{fm}{ }^{3}$ and $V_{0}=$ $-225 \mathrm{MeV} \mathrm{fm}^{3}$, respectively. The zero-quasi-particle states (no blocking) are presented for the mercury and gold isotopes (for $V_{0}=-200 \mathrm{MeV} \mathrm{fm}^{3}$ they are the same as in Fig. 4.6). The one-quasi-particle states (with blocking) are only computed for the (odd- $N$ ) mercury isotopes. Irrespective of the treatment of odd nuclei, the general trends observed with the increase of the pairing strength for the results of Fig. 4.6 are confirmed. For the same nucleus and equilibrium configuration, the quadrupole deformation parameter is reduced and so is its deformation energy, when passing from $V_{0}=-200$ to $-225 \mathrm{MeV} \mathrm{fm}^{3}$. The two-neutron separation energies are systematically lower than the ones obtained for $V_{0}=-200 \mathrm{MeV} \mathrm{fm}^{3}$. The extension of the region of large prolate deformation is further reduced, as well as the part of it which belongs to the global energy minimum and to hence the nuclear ground state.

One observes in Figs. 4.10(c) and 4.10(d) that the implementation of quasi-particle 


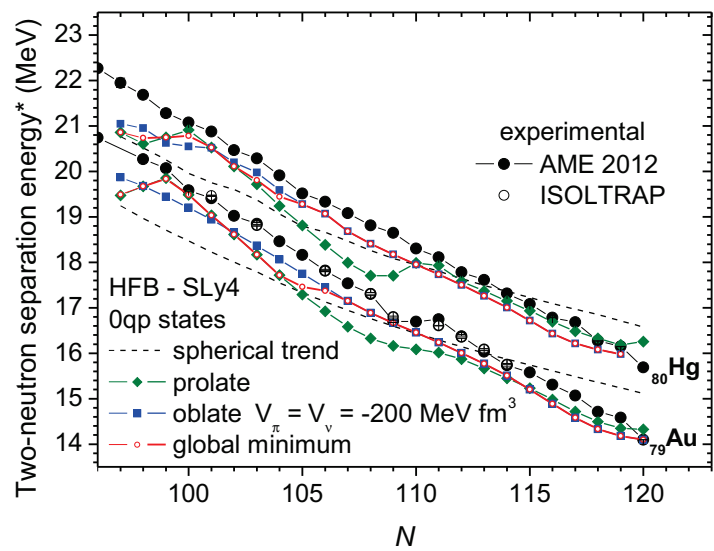

(a)

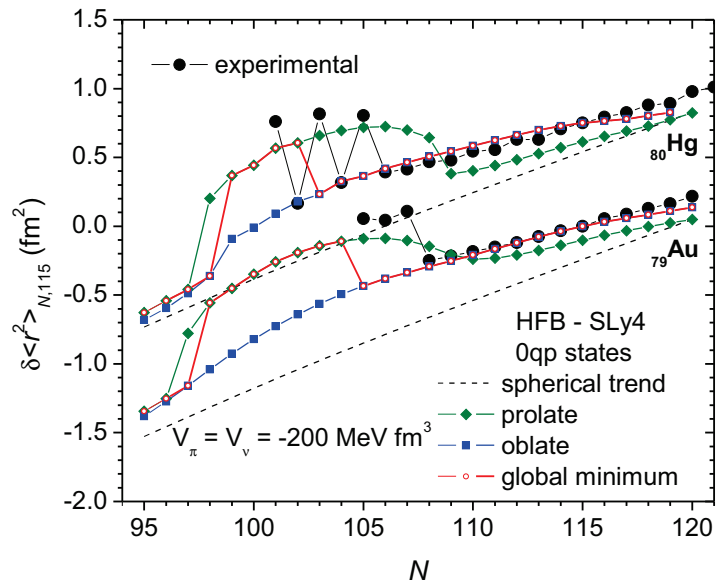

(c)

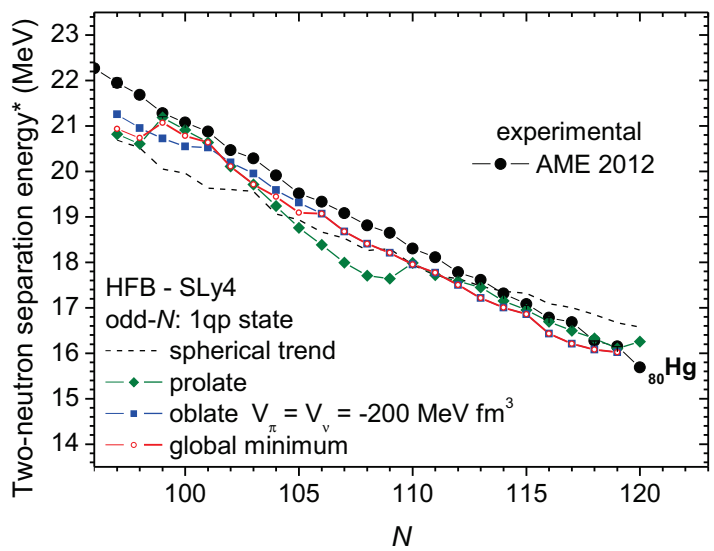

(b)

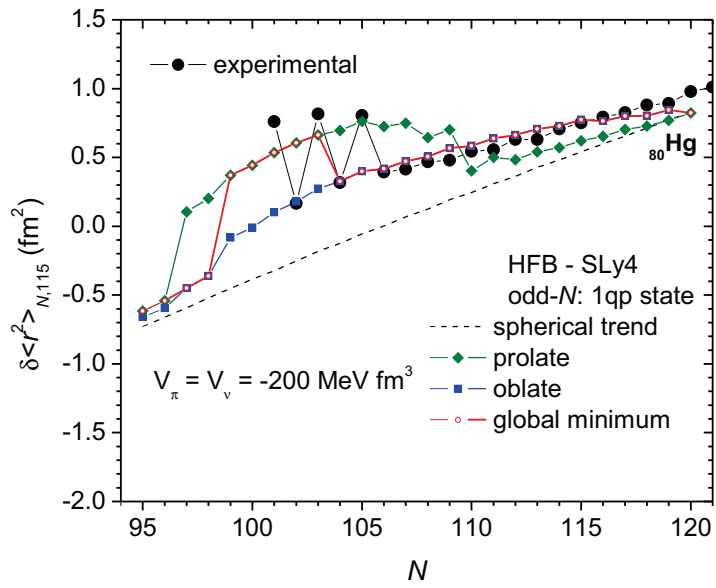

(d)

Figure 4.10: Experimental two-neutron separation energies and mean-square charge radii of neutron-deficient gold and mercury isotopes, compared to HFB-SLy4 calculations using a pairing interaction of strength $V_{0}=-200 \mathrm{MeV} \mathrm{fm}{ }^{3}$. The experimental $S_{2 n}$ values use masses from the AME2012 [Wan12] and this work. The $\left\langle\delta r^{2}\right\rangle$ values are taken from [Fri04a; Fri04b]. The left panels show theoretical results for the zero-quasi-particle states of gold and mercury isotopes (without blocking). The right panels show results for the mercury isotopes, with the odd- $N$ ones computed as one-quasi-particle states (with blocking). The results for the spherical, minimumenergy oblate and minimum-energy prolate configurations are shown separately (dashed line, blue squares and green diamonds, respectively). The red empty circles represent the values corresponding to the ground state, assigned to the configuration of lowest energy. The $S_{2 n}$ and $\left\langle\delta r^{2}\right\rangle$ of the mercury isotopes are biased for better separation. 


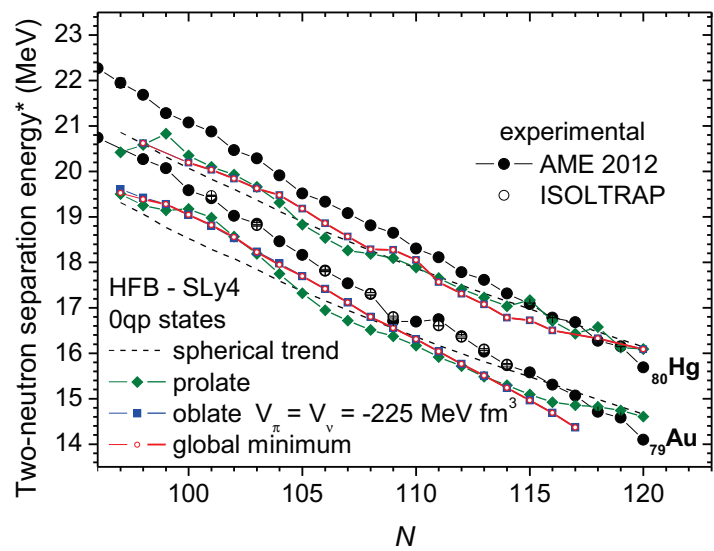

(a)

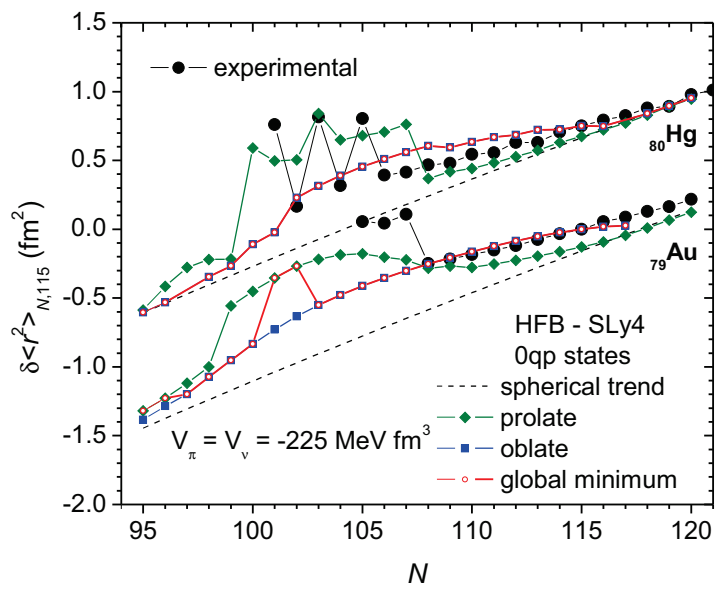

(c)

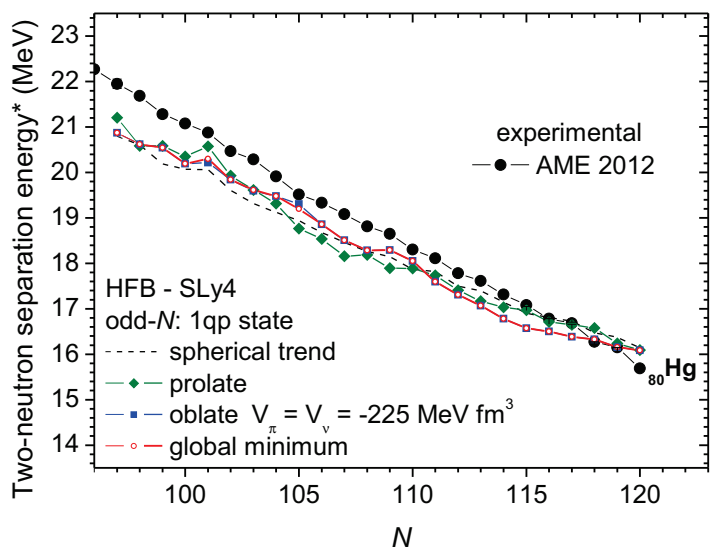

(b)

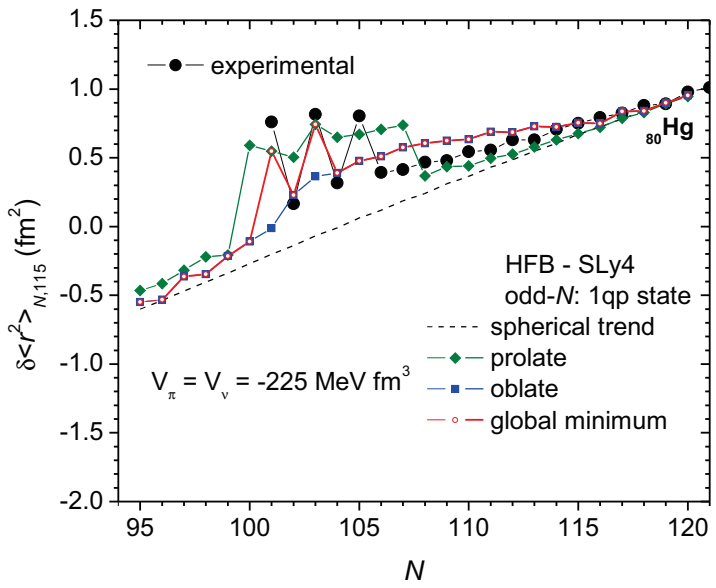

(d)

Figure 4.11: Experimental two-neutron separation energies and mean-squared charge radii of neutron-deficient gold and mercury isotopes, compared to HFB-SLy4 calculations using a pairing interaction of strength $V_{0}=-225 \mathrm{MeV} \mathrm{fm}{ }^{3}$. The experimental $S_{2 n}$ values use masses from the AME2012 [Wan12] and this work. The $\left\langle\delta r^{2}\right\rangle$ values are taken from [Fri04a; Fri04b]. The left panels show theoretical results for the zero-quasi-particle states of gold and mercury isotopes (without blocking). The right panels show results for the mercury isotopes, with the odd- $N$ ones computed as one-quasi-particle states (with blocking). The results for the spherical, minimumenergy oblate and minimum-energy prolate configurations are shown separately (dashed line, blue squares and green diamonds, respectively). The red empty circles represent the values corresponding to the ground state, assigned to the configuration of lowest energy. The $S_{2 n}$ and $\left\langle\delta r^{2}\right\rangle$ of the mercury isotopes are biased for better separation. 
blocking modifies both the extension of the minimum of large deformation among the prolate configurations and the number of isotopes in which this minimum gives the nuclear ground-state. As shown in Figs. 4.11(a) and 4.11(c), for $V_{0}=-225 \mathrm{MeV} \mathrm{fm}^{3}$ and no blocking, only two gold isotopes are still predicted to have the large prolate deformation, while in the case of mercury the oblate configuration is predicted to persist as the ground state throughout the entire range of calculated isotopes, at odds with the experimental values. Once blocking is implemented, the energy balance between the large-deformation prolate configuration and the oblate configuration changes in the odd- $N$ systems for $98 \leq$ $N \leq 104$, due to the fact that, with respect to the fully-paired solution, the oblate and the prolate configurations lose different amounts of binding energy by the blocking of the odd neutron. This is reflected in Figs. 4.8(a) and 4.8(b) by the fact that the $\Delta_{5 n}$ values differ significantly between the prolate and oblate configuration for this range. The consequence of this phenomenon are the successive changes of the ground state between oblate and prolate deformation predicted for $V_{0}=-225 \mathrm{MeV} \mathrm{fm}^{3}$. The result, shown in Fig. 4.11(d), is that the ground-state mean-square charge radii exhibit the pronounced odd-even staggering which is observed in experiment.

\subsection{Summary}

The calculations presented in this chapter represent an exploration of the mean-field landscape in two of the most pronounced shape-transition regions of the nuclear chart: neutron-rich $A \approx 100$ and neutron-deficient gold-thallium isotopes. Although still a large simplification of the nuclear many-body problem, the (deformed) mean-field approximation gives the leading-order contribution to the description of nuclear structure in mid-shell regions of the nuclear chart. In particular, it captures the competition between paring and quadrupole correlations, which are the two main driving forces for the evolution of nuclear structure in open-shell nuclei [Rin00; Cas05]. Thus, despite the limitations inherent to the mean-field approximation and to the modeling of pairing correlations in HFB theory, a few conclusions can be drawn from this analysis, which will be summarized in the following. They also rely on the presented literature results [Ben06; Del10] which, containing both the mean-field and the beyond-mean-field solutions for even-even nuclei, allow discussing the expected effect of dynamic quadrupole correlations on the computed observables.

The mean-field landscape in open-shell nuclei typically consists of more than one equilibrium configuration, each having its smooth, steady evolution with proton and neutron number. Considering that the ground state is given in the mean-field description by the configuration of lowest energy, ground-state shape transitions correspond to changes of the energy ordering between two coexisting configurations. Although sudden when regarding only the ground-state properties, shape transitions are generally smooth phenomena with respect to the full mean-field landscape, i.e. considering all existing equilibrium config- 
urations. Considering also the effect of collective motion, which is frozen at the static mean-field level, shape transitions are related to changes in the mean-field configuration which has the largest weight in the fully-correlated ground state.

Irrespective of the way it is modeled, the pairing functional (or interaction) influences the deformation energy of the oblate and prolate configurations (energy balance with respect to the spherical solution), but also the relative energy balance between them. As such, it has a significant impact on shape-transition phenomena and the resulting signatures (location along an isotopic chain and strength). The proper description of shape transitions poses an important constraint on the particle-particle part of the energy functional, for a certain choice of its particle-hole part. Together with the description of empirical shell gaps, this acts as an additional constraint on the modeling of pairing correlations, complementary to the odd-even staggering of binding energies.

Both for the neutron-rich $A \approx 100$ and for the neutron-deficient gold-thallium isotopes, the calculations attribute the shape-transitions observed in the charge radii at $N \approx 60$ and $N \approx 105-107$, respectively, to a change of the nuclear ground-state between an oblate and a large-deformation prolate minimum of the nuclear mean-field. This is in agreement with results which include beyond-mean-field correlations for even-even nuclei [Ben06]. For the gold isotopes, the experimental $S_{2 n}$ values around $N=107$ agree qualitatively with the type of $S_{2 n}$ evolution predicted by the HFB calculations close to the oblateto-prolate transition in the nuclear ground state. However, one notices that there is a significant displacement between the location of the flattening of $S_{2 n}$ and the signature in the charge radii. A similar signature is predicted for the mercury isotopes in the meanfield calculations of the present work and in the cited literature results [Ben06; Del10], but it is shown in Fig. 4.7 that this signature is washed out by beyond-mean-field correlations. We note however that the calculations of [Ben06; Del10] predict the even-even mercury isotopes in the shape-staggering region to have large deformation, contrary to experiment.

For the neutron-rich $A \approx 100$ nuclei, the excellent agreement with experiment of the prolate configuration taken individually (charge radii and two-neutron separation energies) still allows the observed trends to be explained by a transition between a lowdeformation and a large-deformation prolate minimum. The calculations nevertheless predict an oblate-to-prolate transition at $N \approx 60$ for all investigated strengths of the pairing interaction.

The different behavior of the experimental $S_{2 n}$ and $\left\langle\delta r^{2}\right\rangle$ values in the krypton isotopic chain (with respect to the rubidium isotopic chain), including the new masses presented in this work, can be explained either by a persistence of the oblate configuration in the nuclear ground state or by a transition to large prolate deformation at a higher neutron number than the extension of the available experimental data. The results of [Del10] hint at a third possible explanation, related to the softness of the mean-field solution. Although predicting at the mean-field level a transition to larger deformation already at $N=60$, the beyond-mean-field results of Fig. 4.3 show a reduction of the relative charge radii of 
${ }^{96,98} \mathrm{Kr}$. Further mass and charge-radius (or electric-moment) measurements along the krypton isotopic chain should provide crucial additional information and clarify whether the krypton isotopes represent the boundary of the quantum phase transition in the region.

It is very difficult, with the current modeling of the pairing functional in SkyrmeHFB theory, to find a pairing strength which provides a reasonable description of both shape-transition phenomena and the odd-even staggering of binding energies, for the two investigated regions of the nuclear chart. Although their systematic study was limited to the HFB quasi-particle vacuum, without any blocking prescription or configuration mixing, the description of two-neutron separation energies and mean-square charge radii of the mid-shell nuclei in the HFB-SLy4 approach seems to require a volume-pairing strength closer to $V_{0}=-175 \mathrm{MeV} \mathrm{fm}^{3}$.

Stronger pairing $\left(V_{0}=-200 \mathrm{MeV} \mathrm{fm}^{3}\right)$ leads to an underestimation of the strength of the $S_{2 n}$ signature in the nuclear ground states of the neutron-rich $A \approx 100$ nuclei, determined by the $N \approx 60$ shape transition. It also leads to systematically lower theoretical $S_{2 n}$ values with respect to experiment for the neutron-deficient gold-thallium nuclei. In what concerns the $N=50$ two-neutron shell gap, although it is not properly described at the static mean-field level, stronger pairing gives a very low mean-field approximation for its trend with proton number. One notes however the remarkable description of the experimental $S_{2 n}$ signature around $N=60$ by the prolate configuration of the mean field, taken individually. The agreement is best, in this sense, for $V_{0}=-200 \mathrm{MeV} \mathrm{fm}$.

Based on results from the literature which include the effect of collective motion through beyond-mean-field techniques [Ben06; Del10], the addition of dynamic correlations produces a change which further disfavors a stronger pairing interaction. Especially referring to the neutron-rich $A \approx 100$ nuclei, configuration mixing would lead to an even smoother $S_{2 n}$ trend around the $N=60$ shape transition and to an increase of the charge radii of the reference $N=50$ nuclei. This in turn would lead to lower $\left\langle\delta r^{2}\right\rangle$ values for $N>60$ than in the static mean-field approximation, as shown in Fig. 4.3. Finally, the effect of dynamic correlations on the $N=50$ two-neutron shell gap, illustrated in Fig. 4.4(b), is of a systematic reduction at every proton number. For all these subsequent effects which emerge at the beyond-mean-field level, a pairing strength $V_{0}=-200 \mathrm{MeV} \mathrm{fm}{ }^{3}$ or stronger seems to provide unfavorable mean-field starting conditions.

Describing odd nuclei as one-quasi-particle states in the mean-field approximation, a stronger volume-pairing interaction than $V_{0}=-200 \mathrm{MeV} \mathrm{fm}{ }^{3}$ is necessary, as shown in Fig. 4.9, in order to obtain the proper magnitude of the odd-even staggering of binding energies along the mercury isotopic chain (as a result of blocking). A value of the pairing strength $V_{0}=-175 \mathrm{MeV} \mathrm{fm}^{3}$ significantly underestimates the experimental $\Delta_{5 n}$ values.

The magnitude of the odd-even staggering of binding energies as a result of blocking generally differs between the competing spherical and deformed configurations. The energy balance between the deformed configurations at the level of the HFB quasi-particle vacuum can change when blocking is implemented and with it the configuration which 
gives the mean-field ground state. The odd-even staggering of charge radii in the mercury isotopic chain is likely the reflection of this phenomenon: the competition between two mean-field configurations, the ordering of which is different for the fully-paired HFB states (even- $N$ isotopes) than for the blocked one-quasi-particle states (odd- $N$ isotopes). Since the difference in empirical pairing gap between the competing configurations can be as large as $0.5 \mathrm{MeV}$, the final ordering between them cannot be unambiguously discussed before quasi-particle blocking is implemented. This is especially important if the binding energies of the different configurations differ by less than this amount for their fully-paired vacua. When studying the systematics with neutron number of odd- $Z$ isotopic chains, this problem must be considered for every isotope, unless the fully-paired minima are well separated in energy.

The observed effect of configuration mixing (for even-even nuclei) and quasi-particle blocking (for odd and odd-odd nuclei) in self-consistent mean-field theories imposes a full beyond-mean-field treatment of these isotopes before their properties can be used to unambiguously constrain the parameters of the effective nucleon-nucleon interaction (or energy density functional). The quenching of correlations in doubly-magic nuclei recommends their ground-state properties as a convenient way out for the particle-hole part of the interaction, although it has been pointed out that, because the fitting is performed in the mean-field approximation, the nuclei used for the fit are systematically over-bound once configuration-mixing is implemented [Was12].

In what concerns the particle-particle part of the interaction, even if one uses only the odd-even staggering of binding energies, its adjustment requires the computation of midshell nuclei (at least in one nucleon species), for which dynamic quadrupole correlations are non-negligible. This leads to complications, to which one adds the ambiguity resulting when polarization effects are not self-consistently taken into account [Rut99; Ben00; Dug01]. In this context, the effect of the pairing-interaction strength on the description of shape-transition phenomena, through the competition between particle-particle and particle-hole correlations, can be a crucial additional constraint. This can also act as a consistency check between the particle-particle and particle-hole channels of the energy functional. 



\section{Chapter 5}

\section{Conclusions and outlook}

In this work, the masses of neutron-rich rubidium $\left({ }^{98-100} \mathrm{Rb}\right)$ and neutron-deficient gold $\left({ }^{180,185,188,190,191} \mathrm{Au}\right)$ isotopes produced by the ISOLDE facility [Kug00] at CERN were studied with the Penning-trap mass spectrometer ISOLTRAP [Muk08]. Their masses were determined by the time-of-flight ion-cyclotron-resonance technique in a Penning trap [Gra80]. The multi-reflection time-of-flight mass spectrometer (MR-TOF MS) of ISOLTRAP [Wol13b] was used as a beam-analysis tool for laser spectroscopy studies of neutron-deficient gold isotopes, with the resonance-ionization laser ion source of ISOLDE [Mar13]. Producing a complete separation of the isobaric contamination for all studied gold isotopes, the MR-TOF MS is a promising tool for measuring the hyperfine structure of radioactive nuclides, either for the determination of nuclear charge radii, spins and electromagnetic moments, or as a means of providing isomerically pure beams by selective ionization.

The trends of nuclear binding energies contain a series of features which can be extracted by use of finite-difference formulas (or mass filters). Once computed, these filters justify the intuition of describing nuclear structure starting from a mean-field (or independent-particle) picture, from which one builds the interacting (correlated) nuclear state by means of a residual interaction [Cau05] or in a variational way, as a mixture of mean-field configurations which minimize the energy by (spontaneously) breaking the symmetries of the nuclear Hamiltonian [Ben03]. Either way, the evolution of nuclear structure in open-shell nuclei is decisively influenced by the competition between short-range (particle-particle) correlations, which drive the nucleus to spherical shape, and long-range (particle-hole) correlations, which drive the spontaneous breaking of rotational symmetry (intrinsic deformation). In mid-shell regions, nuclei are typically quadrupole deformed in the intrinsic frame, while close to magic numbers the spherical stability of the nucleus is preserved. The change between the two types of structure can be either smooth or sudden, the latter case marking the shape-transition phenomenon.

The most intuitive picture of shape transitions is the one obtained in the framework of mean-field theory [Rin00]. The competition between pairing and quadrupole correlations 
translates into the energy balance between different equilibrium configurations of the mean field (local minima with respect to small variations of deformation), of which the one lowest in energy is associated to the nuclear ground state. With the change of proton and neutron number, the energy difference between these configurations changes, sometimes leading to a change of the ground-state configuration. In turn this determines a change of the trends of ground-state mass filters and mean-square charge radii.

The measured rubidium and gold isotopes cross two of the most prominent shapetransition regions of the nuclear chart. As such, the properties of these nuclides are sensitive to the balance between pairing and quadrupole correlations in the nuclear mean field. A Skyrme-Hartree-Fock-Bogoliubov study of the binding energies and mean-square charge radii of nuclei in the two concerned regions was thus performed [Rin00]. The HFODD code [Dob97; Sch12] was used for calculations, with the SLy4 interaction for the particle-hole part [Cha98] and a delta volume-pairing interaction for the particle-particle part of the energy functional [Ben03]. Most calculations of odd nuclei were performed only at the level of the HFB quasi-particle vacuum [Dug01], except for the odd- $N$ mercury isotopes, which were also computed with full quasi-particle blocking [Dob09b; Sch10], allowing the determination of their odd-even staggering.

As a general result, the study revealed that the nuclear mean-field exhibits oblate as well as prolate equilibrium configurations, lying close together in energy, for both the rubidium and gold isotopes. This is in agreement with other results from the literature, such as global HFB calculations using the Gogny interaction [Del10]. Close to stability, the oblate configuration is favored in energy and determines a smooth variation of the binding energies and mean-square charge radii. Towards mid-shell, the prolate configuration undergoes a transition from low to large deformation and, by the gradual gain in binding energy, becomes the ground-state configuration. The position of this transition, as well as the way it is reflected in the ground-state observables, depends on the strength of the pairing interaction. The pairing interaction also influences significantly the predicted magnitude of the odd-even staggering of binding energies, as shown for the mercury isotopes. This means that the odd-even staggering effect and the locations of shape transitions can be used together to consistently constrain the pairing interaction, which is usually fitted to reproduce only the first of the two observables. In particular, it is shown that, for a volume-type, delta pairing interaction, it is difficult to find a strength which allows the simultaneous description of two-neutron separation energies across the studied shape-transition points (requiring a value lower than $200 \mathrm{MeV} \mathrm{fm}^{3}$ ) and the odd-even staggering of neutron-deficient mercury isotopes (requiring a value higher than $200 \mathrm{MeV} \mathrm{fm}^{3}$ ).

The experimental two-neutron separation energies of the rubidium isotopes were extended to $N=63$ and confirm the persistence of static deformation in the rubidium isotopic chain up to this neutron number, further than can be concluded based on measurements of mean-square charge-radii. The calculations are in agreement with this pic- 
ture, predicting a transition from oblate to prolate deformation around $N=60$, which persists in the ground state towards $N=66$. The different behavior of $S_{2 n}$ and meansquared charge radii in the krypton isotopic chain [Nai10] can be attributed either to a persistence of oblate deformation in the nuclear ground-state, or to a transition to prolate deformation at a larger neutron number than so-far reached by experiments.

The ISOLTRAP measurements of neutron-deficient gold isotopes refine the mass surface before and after the shape transition which takes place at $N=107$. The determined masses of ${ }^{188,190} \mathrm{Au}$ deviate significantly from the literature values. The new $S_{2 n}$ values have a smoother trend around the transition point, but a drop of $S_{2 n}$ between $N=108$ and 109 is still observed, as well as a flattening of the $S_{2 n}$ slope between $N=109$ and 111. The HFB calculations predict the shape transition to take place in the gold chain between an oblate and a large-deformation prolate configuration, although at a systematically lower neutron number than experimentally observed. A flattening of the $S_{2 n}$ slope is shown to occur close to the transition point in the mean-field approximation. The return to a configuration of lower deformation is predicted for $N<100$, which should be clearly observed in the charged radii of the more neutron-deficient gold isotopes. A signature in $S_{2 n}$ is predicted, but not observed in the experimental values, which can be due to configuration mixing.

The calculations without quasi-particle blocking predict that the oblate-to-prolate transition also takes place in the mercury isotopic chain, for a pairing-interaction strength below $-200 \mathrm{MeV} \mathrm{fm}^{3}$. The difference in binding between the oblate and prolate configurations decreases however with the increase of the pairing strength and for $-225 \mathrm{MeV} \mathrm{fm}^{3}$ the oblate configuration is predicted to remain the most bound along the entire mercury isotopic chain. The oblate and prolate configurations lose different amounts of binding by the blocking of an odd neutron. For a pairing-interaction strength of $-225 \mathrm{MeV} \mathrm{fm}{ }^{3}$, this additional effect leads to the alternation of the ground-state configuration between oblate and prolate when passing from even to odd isotopes, respectively. This alternation explains the large odd-even staggering of charge radii for $N<106$ in the mercury isotopic chain.

The study of the two shape-transition regions performed in this work can continue by extending the mass and isotope-shift measurements along the krypton and gold isotopic chains. Further measurements of neutron-rich krypton isotopes would bring clarification on whether they are the boundary of the shape transition in the neutron-rich $A \approx 100$ nuclei [Nai10]. Measurements of more neutron-deficient gold isotopes would explore the transition back to less deformed shapes in a region near the proton drip line. The study of these exotic nuclides with lower yields and half-lives requires faster purification and measurement techniques, and ISOLTRAP's multi-reflection time-of-flight mass spectrometer has proven to be a promising solution for both requirements [Wol12; Wol13b]. Mass measurements with the MR-TOF MS [Wie13] and resonance-ionization laser spectroscopy with MR-TOF separation and detection [Kre13; Mar13] are intriguing developments to 
pursue.

The study of nuclei in mean-field theory is also required to improve in order to give a reliable description of nuclear-structure phenomena. As shown in the present work, the energy balance between mean-field configurations depends on the interplay between particle-particle, particle-hole correlations and blocking. Only the complete treatment of these contributions allows unambiguous conclusions to be drawn about the meanfield landscape. Additionally, a global, beyond-mean-field description of odd nuclei is still lacking and is necessary in order provide the correct laboratory-frame description, including the important effect of configuration mixing [Ben03]. Very recently, renewed efforts in this direction have been published, but are still limited to only a few cases [Bal14]. 
Appendices 



\section{Appendix A}

\section{Finite-difference operators}

In this appendix we make a brief introduction of the most common finite difference operators used in analyzing the trends of nuclear binding energies. The discussion is however general, referring to any continuous, infinitely derivable function $F(X)$. After the results of this subsection are computed, they can be easily transposed to the quantities that the physics discussion requires. The function $F(X)$ needs not have only one argument, however its expansion will only be performed with respect to one, the other hidden arguments being considered fixed to some value.

The function $F(X)$ can be expanded in powers of $X$ around a certain value $X_{0}$ as:

$$
F(X)=\sum_{n=0}^{\infty} \frac{1}{n !}\left(\frac{\partial^{n} F}{\partial X^{n}}\right)_{X=X_{0}}\left(X-X_{0}\right)^{n} .
$$

The elementary finite-difference operators are the simple two-point formulas of step $n, \mathcal{D}_{n}$. Applied to $F(X)$, they give:

$$
\mathcal{D}_{n} F(X)=F(X-n)-F(X) .
$$

It is easy to prove the following relationship:

$$
\mathcal{D}_{n} F(X)=\mathcal{D}_{1}\left[\sum_{i=0}^{n-1} F(X-i)\right] .
$$

The higher-order finite-difference operators $\mathcal{D}_{n}^{k}$ are obtained as powers of the elementary operators $\mathcal{D}_{n}$ :

$$
\mathcal{D}_{n}^{k} F(X)=\left(\mathcal{D}_{n}\right)^{k} F(X) .
$$

Equations (A.3) and (A.4) show that any type of differential operator with which we are concerned can be constructed from the single-step two-point formula $\mathcal{D}_{1}$. 
The general result of applying the finite-difference operator $\mathcal{D}_{n}^{k}$ to $F(X)$ is:

$$
\mathcal{D}_{n}^{k} F(X)=\sum_{i=0}^{k}\left(\begin{array}{c}
k \\
i
\end{array}\right)(-1)^{i} F[X-n(k-i)] .
$$

In order to obtain the simplest formulas for the actions of the different finite-difference operators, we perform in Eq. (A.5) the Taylor expansion of $F[X-n(k-i)]$ around $X-n k / 2$. For odd $k$, this means that the Taylor expansion is performed around a half-integer number. The resulting formula for the action of the operator $\mathcal{D}_{n}^{k}$ is:

$$
\begin{aligned}
\mathcal{D}_{n}^{k} F(X) & =\sum_{i=0}^{k}\left(\begin{array}{c}
k \\
i
\end{array}\right)(-1)^{i} \sum_{j=0}^{\infty} \frac{1}{j !}\left(\frac{\partial^{j} F}{\partial X^{j}}\right)_{X_{0}-n \frac{k}{2}}(n)^{j}\left(i-\frac{k}{2}\right)^{j} \\
& =\sum_{j=1}^{\infty} \frac{(n)^{j}}{j !}\left(\frac{\partial^{j} F}{\partial X^{j}}\right)_{X_{0}-n \frac{k}{2}} \sum_{i=0}^{k}\left(\begin{array}{c}
k \\
i
\end{array}\right)(-1)^{i}\left(i-\frac{k}{2}\right)^{j}
\end{aligned}
$$

where we have already taken into account that the $j=0$ term of the Taylor series, $F(X-n k / 2)$, is always canceled by the finite-difference operator.

Now one can evaluate the sum over $i$ and compute the result of the action of different finite-difference operators on $F(X)$. It is useful to specify some of the results which are relevant for the physics discussion:

$$
\begin{aligned}
& \mathcal{D}_{1} F\left(X_{0}\right)=F\left(X_{0}-1\right)-F\left(X_{0}\right) \\
&=\sum_{j=1}^{\infty} \frac{1}{j !}\left(\frac{\partial^{j} F}{\partial X^{j}}\right)_{X_{0}-\frac{1}{2}}\left[\left(-\frac{1}{2}\right)^{j}-\left(\frac{1}{2}\right)^{j}\right] \\
&=-\left(\frac{\partial F}{\partial X}\right)_{X_{0}-\frac{1}{2}}-\frac{1}{24}\left(\frac{\partial^{3} F}{\partial X^{3}}\right)_{X_{0}-\frac{1}{2}}-\ldots \\
& \mathcal{D}_{1}^{2} F\left(X_{0}\right)=F\left(X_{0}-2\right)-2 F\left(X_{0}-1\right)+F\left(X_{0}\right) \\
&=\sum_{j=1}^{\infty} \frac{1}{j !}\left(\frac{\partial^{j} F}{\partial X^{j}}\right)_{X_{0}-1}\left[(-1)^{j}+1\right] \\
&=\left(\frac{\partial^{2} F}{\partial X^{2}}\right)_{X_{0}-1}+\frac{1}{12}\left(\frac{\partial^{4} F}{\partial X^{4}}\right)_{X_{0}-1}+\ldots \\
& \mathcal{D}_{1}^{3} F\left(X_{0}\right)=F\left(X_{0}-3\right)-3 F\left(X_{0}-2\right)+3 F\left(X_{0}-1\right)-F\left(X_{0}\right) \\
&=\sum_{j=1}^{\infty} \frac{1}{j !}\left(\frac{\partial^{j} F}{\partial X^{j}}\right)_{X_{0}-\frac{3}{2}}\left[\left(-\frac{3}{2}\right)^{j}-3\left(-\frac{1}{2}\right)^{j}+3\left(\frac{1}{2}\right)^{j}-\left(\frac{3}{2}\right)^{j}\right] \\
&=-\left(\frac{\partial^{3} F}{\partial X^{3}}\right)_{X_{0}-\frac{3}{2}}-\frac{1}{8}\left(\frac{\partial^{3} F}{\partial X^{3}}\right)_{X_{0}-\frac{3}{2}}-\ldots
\end{aligned}
$$




$$
\begin{aligned}
\mathcal{D}_{1}^{4} F\left(X_{0}\right)= & F\left(X_{0}-4\right)-4 F\left(X_{0}-3\right)+6 F\left(X_{0}-2\right)-4 F\left(X_{0}-1\right)+F\left(X_{0}\right) \\
= & \sum_{j=1}^{\infty} \frac{1}{j !}\left(\frac{\partial^{j} F}{\partial X^{j}}\right)_{X_{0}-2}\left[(-2)^{j}-4(-1)^{j}-4+(2)^{j}\right] \\
= & \left(\frac{\partial^{4} F}{\partial X^{4}}\right)_{X_{0}-2}+\frac{1}{6}\left(\frac{\partial^{6} F}{\partial X^{6}}\right)_{X_{0}-2}+\ldots \\
\mathcal{D}_{2} F\left(X_{0}\right) & =F\left(X_{0}-2\right)-F\left(X_{0}\right) \\
& =\sum_{j=1}^{\infty} \frac{2^{j}}{j !}\left(\frac{\partial^{j} F}{\partial X^{j}}\right)_{X_{0}-1}\left[\left(-\frac{1}{2}\right)^{j}-\left(\frac{1}{2}\right)^{j}\right] \\
& =-2\left(\frac{\partial F}{\partial X}\right)_{X_{0}-1}-\frac{1}{3}\left(\frac{\partial^{3} F}{\partial X^{3}}\right)_{X_{0}-1}-\ldots \\
\mathcal{D}_{2}^{2} F\left(X_{0}\right) & =F\left(X_{0}-4\right)^{\infty}-2 F\left(X_{0}-2\right)+F\left(X_{0}\right) \\
& =\sum_{j=1}^{\infty} \frac{2^{j}}{j !}\left(\frac{\partial^{j} F}{\partial X^{j}}\right)_{X_{0}-1}\left[(-1)^{j}+1\right] \\
& =4\left(\frac{\partial^{2} F}{\partial X^{2}}\right)_{X_{0}-2}+\frac{4}{3}\left(\frac{\partial^{4} F}{\partial X^{4}}\right)_{X_{0}-2}+\ldots
\end{aligned}
$$





\section{Appendix B}

\section{Motion of a charged particle in a Penning trap}

In the following, the equations of motion of a charged particle in a Penning trap will be solved explicitly. The confining forces acting on the particle of mass $m$ and charge $q$ are generated by the superposition of a quadrupole electrostatic field and a homogeneous magnetic field. The electrostatic field is defined in Cartesian coordinates $\{\hat{x}, \hat{y}, \hat{z}\}$ by the potential:

$$
V=V_{0} \frac{z^{2}-\frac{r^{2}}{2}}{2 d^{2}}
$$

where $r^{2}=x^{2}+y^{2}$ and $d$ is the characteristic trap dimension:

$$
d^{2}=\frac{1}{2}\left(z_{0}^{2}+r_{0}^{2} / 2\right)
$$

with $z_{0}$ and $r_{0}$ the minimum distances between the center of the trap and the endcap and ring electrodes, respectively (see Fig. 2.1(a)). With this choice of distances, $V_{0}$ is the potential difference between the endcaps and the ring electrode. The electric force resulting from the potential is given by:

$$
F_{e}=-q \nabla V=\frac{q V_{0}}{2 d^{2}} \mathbf{r}-\frac{z q V_{0}}{d^{2}} \hat{z}
$$

where $\mathbf{r}$ is the radial position vector of coordinates $(x, y)$.

The magnetic field acts on the particle through the Lorentz force. Taking the field counter-parallel to the axis $\hat{z}, \mathbf{B}=-B \hat{z}$, the magnetic force is given by:

$$
F_{m}=q \dot{\mathbf{r}} \times \mathbf{B}=q B \hat{z} \times \dot{\mathbf{r}} .
$$

The total force acting on the charged particle can be decomposed into an axial and a radial component:

$$
F_{z}=-\frac{z q V_{0}}{d^{2}} \hat{z}
$$


and

$$
F_{r}=\frac{q V_{0}}{2 d^{2}} \mathbf{r}+q B \hat{z} \times \dot{\mathbf{r}}
$$

The axial and radial dynamics are completely decoupled, leading to two independent equations of motion:

$$
\ddot{z}+\frac{z q V_{0}}{m d^{2}} \hat{z}=0
$$

and

$$
\ddot{\mathbf{r}}-\frac{q V_{0}}{2 m d^{2}} \mathbf{r}+\frac{q B}{m} \hat{z} \times \dot{\mathbf{r}}=0 .
$$

The first is the equation of a harmonic oscillator of (axial) frequency:

$$
\omega_{z}=\sqrt{\frac{q V_{0}}{m d^{2}}},
$$

meaning that the axial motion of the particle in the Penning trap is simply the harmonic oscillation around the trap center at a characteristic frequency, depending on the particle's mass and on the trapping potential.

Before solving the radial equation of motion, it is worth recognizing that $q B / m$ is the particle's cyclotron frequency $\omega_{c}$ in the magnetic field. Although not physically relevant for the radial motion, for compactness one can also substitute the axial frequency in Eq. (B.8) to obtain:

$$
\ddot{\mathbf{r}}-\frac{\omega_{z}^{2}}{2} \mathbf{r}+\omega_{c} \hat{z} \times \dot{\mathbf{r}}=0 .
$$

Equation (B.10) is in Cartesian coordinates a set of two coupled, linear, homogeneous differential equations, of second order. To put the set of equations in canonical form, one expands the cross product and writes the system of equations in matrix form:

$$
\begin{aligned}
\left(\begin{array}{l}
\ddot{x} \\
\ddot{y}
\end{array}\right) & =\frac{\omega_{z}^{2}}{2}\left(\begin{array}{ll}
1 & 0 \\
0 & 1
\end{array}\right)\left(\begin{array}{l}
x \\
y
\end{array}\right)+\omega_{c}\left(\begin{array}{cc}
0 & -1 \\
1 & 0
\end{array}\right)\left(\begin{array}{l}
\dot{x} \\
\dot{y}
\end{array}\right) \\
\ddot{\mathbf{r}} & =A \mathbf{r}+B \dot{\mathbf{r}} .
\end{aligned}
$$

To reduce the order of the equations, one makes the substitution $\dot{\mathbf{r}}=\mathbf{v}$ which allows turning Eq. (B.11) into a system of four linear, homogeneous, differential equations of first order:

$$
\begin{aligned}
\dot{\mathbf{r}} & =\mathbf{v} \\
\dot{\mathbf{v}} & =A \mathbf{r}+B \mathbf{v} .
\end{aligned}
$$

Equation (B.12) can also be written in a compact matrix form as:

$$
\left(\begin{array}{c}
\dot{\mathbf{r}} \\
\dot{\mathbf{v}}
\end{array}\right)=\left(\begin{array}{cc}
0 & I \\
A & B
\end{array}\right)\left(\begin{array}{c}
\mathbf{r} \\
\mathbf{v}
\end{array}\right)
$$


where 0 and $I$ are the null and identity matrices, respectively. Because the system of Eq. (B.13) has dimension four, its general solution is of the form:

$$
\sum_{k=1}^{4} e^{\lambda_{k} t} \mathbf{u}_{k}
$$

where $\lambda_{k}$ are the eigenvalues of the $4 \times 4$ matrix of Eq. (B.13) and $\mathbf{u}_{k}$ are a corresponding set of independent eigenvectors. Using generic notations for the components of $\mathbf{u}$, the eigenvalue problem is formulated as:

$$
\left(\begin{array}{cc}
0 & I \\
A & B
\end{array}\right)\left(\begin{array}{l}
\mathbf{p} \\
\mathbf{q}
\end{array}\right)=\lambda\left(\begin{array}{l}
\mathbf{p} \\
\mathbf{q}
\end{array}\right),
$$

where $\mathbf{p}$ and $\mathbf{q}$ are each $2 \mathrm{D}$ vectors. From the first row of the matrix, one gets that $\mathbf{q}=\lambda \mathbf{p}$. Using this information, from the second row of the matrix one obtains the equation:

$$
A \mathbf{p}+\lambda B \mathbf{p}=\lambda^{2} \mathbf{p}
$$

In Eq. (B.16), p is a $\mathbf{r}$-like vector, of components labeled for simplicity by $c$ and $d$. Using the expressions of $A$ and $B$ from Eq. (B.12), Eq. (B.16) one obtains the system:

$$
\begin{aligned}
& \left(\frac{\omega_{z}^{2}}{2}-\lambda^{2}\right) c-\lambda \omega_{c} d=0 \\
& \left(\frac{\omega_{z}^{2}}{2}-\lambda^{2}\right) d+\lambda \omega_{c} c=0
\end{aligned}
$$

which, through substitution, leads to a second-order equation for $\lambda^{2}$ :

$$
\left(\lambda^{2}\right)^{2}+\left(\omega_{c}^{2}-\omega_{z}^{2}\right) \lambda^{2}+\frac{\omega_{z}^{4}}{4}=0
$$

The solutions for $\lambda^{2}$ are:

$$
\lambda^{2}=\frac{1}{2}\left(-\omega_{c}^{2}+\omega_{z}^{2} \pm \omega_{c} \sqrt{\omega_{c}^{2}-2 \omega_{z}^{2}}\right)
$$

which are actually given by:

$$
\lambda^{2}=-\omega_{ \pm}^{2}
$$

with

$$
\omega_{ \pm}=\frac{1}{2}\left(\omega_{c} \pm \sqrt{\omega_{c}^{2}-2 \omega_{z}^{2}}\right)
$$

From Eq. (B.21) one can verify the following two relationships:

$$
\begin{aligned}
& \omega_{c}=\omega_{+}+\omega_{-}, \\
& \omega_{c}^{2}=\omega_{+}^{2}+\omega_{-}^{2}+\omega_{z}^{2} .
\end{aligned}
$$


The four values of $\lambda$ resulting from Eq. (B.20) are $\pm i \omega_{ \pm}$. Substituting back in Eq. (B.17), one can find for each value of $\lambda$ the corresponding family of eigenvectors u:

$$
\begin{gathered}
\lambda_{++}=i \omega_{+}, \quad \mathbf{p}_{++}=\left(\begin{array}{c}
i \omega_{+} \omega_{c} \\
\frac{\omega_{z}^{2}}{2}+\omega_{+}^{2}
\end{array}\right)=\left(\begin{array}{l}
i \\
1
\end{array}\right), \\
\lambda_{+-}=-i \omega_{+}, \quad \mathbf{p}_{+-}=\left(\begin{array}{c}
-i \omega_{+} \omega_{c} \\
\frac{\omega_{z}^{2}}{2}+\omega_{+}^{2}
\end{array}\right)=\left(\begin{array}{l}
1 \\
i
\end{array}\right), \\
\lambda_{-+}=i \omega_{-}, \quad \mathbf{p}_{-+}=\left(\begin{array}{c}
i \omega_{-} \omega_{c} \\
\frac{\omega_{z}^{2}}{2}+\omega_{-}^{2}
\end{array}\right)=\left(\begin{array}{l}
i \\
1
\end{array}\right), \\
\lambda_{--}=-i \omega_{-}, \quad \mathbf{p}_{--}=\left(\begin{array}{c}
-i \omega_{-} \omega_{c} \\
\frac{\omega_{z}^{2}}{2}+\omega_{-}^{2}
\end{array}\right)=\left(\begin{array}{l}
1 \\
i
\end{array}\right),
\end{gathered}
$$

where one has used:

$$
\frac{\omega_{z}^{2}}{2}+\omega_{ \pm}^{2}=\omega_{ \pm} \omega_{c}
$$

easy to show from Eq. (B.22).

The general solution can be obtained using Eq. (B.14). Since one is not interested by the velocity, Eq. (B.14) can be written only for the $\mathbf{r}$ part, using instead of $\mathbf{u}$ the $\mathbf{p}$ vectors of Eq. (B.23). To also construct a real solution, one picks of the pairs of complexconjugate eigenvalues $\lambda$ only one and uses of the corresponding $e^{\lambda t} \mathbf{p}$ product the real and the imaginary parts as independent eigenvectors:

$$
\begin{aligned}
\mathbf{r} & =C_{1} \Re\left(e^{\lambda_{++} t} \mathbf{p}_{++}\right)+C_{2} \Im\left(e^{\lambda_{++} t} \mathbf{p}_{++}\right) \\
& +C_{3} \Re\left(e^{\lambda_{-+}} \mathbf{p}_{-+}\right)+C_{4} \Im\left(e^{\lambda_{-+} t} \mathbf{p}_{-+}\right)
\end{aligned}
$$

Using the values obtained in Eq. (B.23), the general solution becomes:

$$
\begin{aligned}
\mathbf{r} & =C_{1}\left(\begin{array}{c}
-\sin \left(\omega_{+} t\right) \\
\cos \left(\omega_{+} t\right)
\end{array}\right)+C_{2}\left(\begin{array}{c}
\cos \left(\omega_{+} t\right) \\
\sin \left(\omega_{+} t\right)
\end{array}\right) \\
& +C_{3}\left(\begin{array}{c}
-\sin \left(\omega_{-} t\right) \\
\cos \left(\omega_{-} t\right)
\end{array}\right)+C_{4}\left(\begin{array}{c}
\cos \left(\omega_{-} t\right) \\
\sin \left(\omega_{-} t\right)
\end{array}\right) .
\end{aligned}
$$

Instead of the $C$ constants in Equation (B.26), one can rewrite the solution in terms of two amplitudes and two phases. To this end, one performs the following transformations:

$$
\begin{aligned}
& \rho_{+}=\sqrt{C_{1}^{2}+C_{2}^{2}}, \quad \sin \left(\phi_{+}\right)=\frac{C_{1}}{\sqrt{C_{1}^{2}+C_{2}^{2}}}, \quad \cos \left(\phi_{+}\right)=\frac{C_{2}}{\sqrt{C_{1}^{2}+C_{2}^{2}}}, \\
& \rho_{-}=\sqrt{C_{3}^{2}+C_{4}^{2}}, \quad \sin \left(\phi_{-}\right)=\frac{C_{3}}{\sqrt{C_{3}^{2}+C_{4}^{2}}}, \quad \cos \left(\phi_{-}\right)=\frac{C_{4}}{\sqrt{C_{3}^{2}+C_{4}^{2}}} .
\end{aligned}
$$


With these transformations, Eq. (B.26) becomes:

$$
\mathbf{r}=\rho_{+}\left(\begin{array}{c}
\cos \left(\omega_{+} t+\phi_{+}\right) \\
\sin \left(\omega_{+} t+\phi_{+}\right)
\end{array}\right)+\rho_{-}\left(\begin{array}{c}
\cos \left(\omega_{-} t+\phi_{-}\right) \\
\sin \left(\omega_{-} t+\phi_{-}\right)
\end{array}\right)
$$

which is the superposition of two circular motions, one at $\omega_{+}$, called the modified cyclotron frequency, and one at $\omega_{-}$, called the magnetron frequency. 



\section{Appendix C}

\section{Publications related to thesis work}

C. Böhm et al., "Evolution of nuclear ground-state properties of neutron-deficient isotopes around $Z=82$ from precision mass measurements," Phys. Rev. C 90, 044307 (2014).

S. Kreim et al., "Recent exploits of the ISOLTRAP mass spectrometer," Nucl. Instrum. Meth. B 317, 492-500 (2013).

S. Kreim et al., "Competition between pairing correlations and deformation from the odd-even mass staggering of francium and radium isotopes," Phys. Rev. C 90, 024301 (2014).

V. Manea et al., "Collective degrees of freedom of neutron-rich $A \approx 100$ nuclei and the first mass measurement of the short-lived nuclide ${ }^{100} \mathrm{Rb}, "$ Phys. Rev. C 88, 054322 (2013).

B. Marsh et al., "New developments of the in-source spectroscopy method at RILIS/ ISOLDE," Nucl. Instrum. Meth. B 317, 550-556 (2013).

J. Stanja et al., "Mass spectrometry and decay spectroscopy of isomers across the $Z=82$ shell closure," Phys. Rev. C 88, 054304 (2013).

F. Wienholtz et al., "Masses of exotic calcium isotopes pin down nuclear forces," Nature 498, 346 (2013).

R. N. Wolf et al., "Plumbing Neutron Stars to New Depths with the Binding Energy of the Exotic Nuclide ${ }^{82}$ Zn," Phys. Rev. Lett. 110, 041101 (2013).

R. Wolf et al., "ISOLTRAP's multi-reflection time-of-flight mass separator/ spectrometer," Int. J. Mass Spectrom. 349-350, 123-133 (2013). 



\section{Bibliography}

[Abo95] Y. Aboussir, J. Pearson, A. Dutta, F. Tondeur, "Nuclear mass formula via an approximation to the Hartree-Fock method," At. Data Nucl. Data Tables 61, 127-176 (1995).

[Alb12] M. Albers et al., "Evidence for a Smooth Onset of Deformation in the NeutronRich Kr Isotopes," Phys. Rev. Lett. 108, 062701 (2012).

[And00] A. Andreyev et al., "A triplet of differently shaped spin-zero states in the atomic nucleus ${ }^{186} \mathrm{~Pb}, "$ Nature 405, 430 (2000).

[Arn07] M. Arnould, S. Goriely, K. Takahashi, "The r-process of stellar nucleosynthesis: Astrophysics and nuclear physics achievements and mysteries," Phys. Rep. 450, 97 (2007).

[Asc14] Ascher, P. et al., "PIPERADE: A Penning-trap isobar separator for the DESIR low-energy facility of SPIRAL2," EPJ Web of Conferences 66, 11002 (2014).

[Ast19] F. W. Aston, "The Constitution of the Elements," Nature 104, 393 (1919).

[Ast20a] F. W. Aston, "The Mass-Spectra of Chemical Elements," Phil. Mag. 39, 611625 (1920).

[Ast20b] F. W. Aston, "XLIV. The constitution of atmospheric neon," Phil. Mag. 39, 449-455 (1920).

[Ast27] F. W. Aston, "Atoms and their Packing Fractions," Nature 120, 956-959 (1927).

[Aud12] G. Audi et al., "The Nubase2012 evaluation of nuclear properties," Chinese Phys. C 36, 1157 (2012).

[Aud86] G. Audi et al., "Mass-spectrometric measurements of exotic Rb, Cs and Fr isotopes," Nucl. Phys. A 449, 491-518 (1986).

[Bal14] B. Bally, B. Avez, M. Bender, P.-H. Heenen, "Beyond Mean-Field Calculations for Odd-Mass Nuclei," Phys. Rev. Lett. 113, 162501 (2014).

[Ban74] B. Banerjee, P. Ring, H. Mang, "On the character of the Hartree-Fock-Bogoliubov solutions in a rotating frame," Nucl. Phys. A 221, 564-572 (1974). 
[Bar13] A. E. Barzakh et al., "Changes in the mean-square charge radii and magnetic moments of neutron-deficient Tl isotopes," Phys. Rev. C 88, 024315 (2 Aug. 2013).

[Bec09] D. Beck et al., "Electric and magnetic field optimization procedure for Penning trap mass spectrometers," Nucl. Instr. Meth. Phys. Res. A 598, 635-641 (2009).

[Bec90] S. Becker et al., "Mass measurements of very high accuracy by time-of-flight ion cyclotron resonance of ions injected into a penning trap," Int. J. Mass Spectrom. 99, 53-77 (1990).

[Ben00] M. Bender, K. Rutz, P.-G. Reinhard, J. Maruhn, "Pairing gaps from nuclear mean-field models," Eur. Phys. J. A 8, 59-75 (2000).

[Ben03] M. Bender, P.-H. Heenen, P.-G. Reinhard, "Self-consistent mean-field models for nuclear structure," Rev. Mod. Phys. 75, 121-180 (2003).

[Ben06] M. Bender, G. F. Bertsch, P.-H. Heenen, "Global study of quadrupole correlation effects," Phys. Rev. C 73, 034322 (2006).

[Ben08] M. Bender, G. F. Bertsch, P.-H. Heenen, "Collectivity-induced quenching of signatures for shell closures," Phys. Rev. C 78, 054312 (2008).

[Ber09] G. Bertsch, J. Dobaczewski, W. Nazarewicz, J. Pei, "Hartree-Fock-Bogoliubov theory of polarized Fermi systems," Phys. Rev. A 79, 043602 (2009).

[Bet36] H. A. Bethe, R. F. Bacher, "Nuclear Physics A. Stationary States of Nuclei," Rev. Mod. Phys. 8, 82-229 (1936).

[Bha92] M. Bhat, in Nuclear Data for Science and Technology, ed. by S. Qaim, Data extracted using the NNDC On-Line Data Service from the ENSDF database (http://nndc.bnl.gov), file revised as of 28.07.2014. (Springer Berlin Heidelberg, 1992), pp. 817-821.

[Bin91] C. R. Bingham et al., "Identification of ${ }^{185} \mathrm{Pt} \alpha$ activity and study of ${ }^{185} \mathrm{Au} \alpha$ decay," Phys. Rev. C 44, 1208-1211 (1991).

[Bla03] K. Blaum et al., "Recent developments at ISOLTRAP: towards a relative mass accuracy of exotic nuclei below 10"8," J. Phys. B 36, 921 (2003).

[Bla06] K. Blaum, "High-accuracy mass spectrometry with stored ions," Phys. Rep. 425, 1-78 (2006).

[Bla10] K. Blaum, Y. N. Novikov, G. Werth, "Penning traps as a versatile tool for precise experiments in fundamental physics," Contemp. Phys. 51, 149-175 (2010).

[Bla97] F. Blanc et al., "Nuclear Moments and Deformation Change in ${ }^{184} A u^{g, m}$ from Laser Spectroscopy," Phys. Rev. Lett. 79, 2213-2216 (1997). 
[Blo62] C. Bloch, A. Messiah, "The canonical form of an antisymmetric tensor and its application to the theory of superconductivity," Nucl. Phys. 39, 95-106 (1962).

[Blu13] Y. Blumenfeld, T. Nilsson, P. V. Duppen, "Facilities and methods for radioactive ion beam production," Phys. Scripta 2013, 014023 (2013).

[Bog10] S. Bogner, R. Furnstahl, A. Schwenk, "From low-momentum interactions to nuclear structure," Progr. Part. Nucl. Phys. 65, 94-147 (2010).

[Boh09] C. Böhm, Setup of a carbon-cluster laser ion source and the application of the invariance theorem at ISOLTRAP, Diploma thesis, 2009.

[Boh14] C. Böhm et al., "Evolution of nuclear ground-state properties of neutrondeficient isotopes around $Z=82$ from precision mass measurements," Phys. Rev. C 90, 044307 (2014).

[Bol01] G. Bollen, "Mass measurements of short-lived nuclides with ion traps," Nucl. Phys. A 693, 3-18 (2001).

[Bol90] G. Bollen, R. B. Moore, G. Savard, H. Stolzenberg, "The Accuracy of HeavyIon Mass Measurements using Time of Flight-Ion Cyclotron Resonance in a Penning Trap," J. Appl. Phys. 68, 4355 (1990).

[Bol92] G. Bollen et al., "Resolution of nuclear ground and isomeric states by a Penning trap mass spectrometer," Phys. Rev. C 46, R2140-R2143 (1992).

[Bon72] J. Bonn, G. Huber, H.-J. Kluge, L. Kugler, E. Otten, "Sudden change in the nuclear charge distribution of very light mercury isotopes," Phys. Lett. B 38, 308-311 (1972).

[Bon76] J. Bonn, G. Huber, H.-J. Kluge, E. Otten, "Spins, moments and charge radii in the isotopic series ${ }^{181} \mathrm{Hg}^{191} \mathrm{Hg}$," English, Z. Phys. A 276, 203-217 (1976).

[Bou08] E. Bouquerel et al., "Beam purification by selective trapping in the transfer line of an ISOL target unit," Nucl. Instrum. Meth. B 266, 4298-4302 (2008).

[Bre14] N. Bree et al., "Shape Coexistence in the Neutron-Deficient Even-Even ${ }^{182-188} \mathrm{Hg}$ Isotopes Studied via Coulomb Excitation," Phys. Rev. Lett. 112, 162701 (2014).

[Bro06] B. A. Brown, W. A. Richter, "New "USD" Hamiltonians for the sd shell," Phys. Rev. C 74, 034315 (2006).

[Bro13] R. A. Broglia, V. Zelevinsky, Eds., Fifty Years of Nuclear BCS, Pairing in Finite Systems (World Scientific, 2013).

[Bro86] L. S. Brown, G. Gabrielse, "Geonium theory: Physics of a single electron or ion in a Penning trap," Rev. Mod. Phys. 58, 233-311 (1986).

[Buc90] F. Buchinger et al., "Systematics of nuclear ground state properties in ${ }^{78-100} \mathrm{Sr}$ by laser spectroscopy," Phys. Rev. C 41, 2883-2897 (1990). 
[Cak10] R. B. Cakirli, R. F. Casten, K. Blaum, "Correlations of experimental isotope shifts with spectroscopic and mass observables," Phys. Rev. C 82, 061306 (2010).

[Cam02] P. Campbell et al., "Laser Spectroscopy of Cooled Zirconium Fission Fragments," Phys. Rev. Lett. 89, 082501 (2002).

[Cam97] P. Campbell, J. Billowes, I. S. Grant, "The isotope shift of ${ }^{90,91} \mathrm{Zr}$ by collinear ion - laser beam spectroscopy," J. Phys. B 30, 4783 (1997).

[Cas05] R. Casten, Nuclear structure from a simple perspective (Oxford Science Publications, 2005).

[Cas87] R. F. Casten, D. S. Brenner, P. E. Haustein, "Valence $p-n$ interactions and the development of collectivity in heavy nuclei," Phys. Rev. Lett. 58, 658 (1987).

[Cau05] E. Caurier, G. Martínez-Pinedo, F. Nowacki, A. Poves, A. P. Zuker, "The shell model as a unified view of nuclear structure," Rev. Mod. Phys. 77, 427 (2005).

[Cej10] P. Cejnar, J. Jolie, R. F. Casten, "Quantum phase transitions in the shapes of atomic nuclei," Rev. Mod. Phys. 82, 2155-2212 (2010).

[Cha32] J. Chadwick, "Possible Existence of a Neutron," Nature 129, 312 (1932).

[Cha98] E. Chabanat, P. Bonche, P. Haensel, J. Meyer, R. Schaeffer, "A Skyrme parametrization from subnuclear to neutron star densities Part II. Nuclei far from stabilities," Nucl. Phys. A 635, 231-256 (1998).

[Che07] B. Cheal et al., "The shape transition in the neutron-rich yttrium isotopes and isomers," Phys. Lett. B 645, 133-137 (2007).

[Coc32] J. D. Cockcroft, E. T. S. Walton, "Disintegration of Lithium by Swift Protons," Nature 129, 242 (1932).

[Com74] M. B. Comisarow, A. G. Marshall, "Fourier transform ion cyclotron resonance spectroscopy," Chem. Phys. Lett. 25, 282-283 (1974).

[Cwi05] S. Ćwiok, P.-H. Heenen, W. Nazarewicz, "Shape coexistence and triaxiality in the superheavy nuclei," Nature 285, 705-709 (2005).

[Dau84] H. Dautet et al., presented at the International conference on atomic masses and fundamental constants, Seeheim-Jugenheim, Germany (AMCO-7), 1984.

[Dau86] H. Dautet, R. Turcotte, S. N. Mark, Annual report of McGill University, Canada (1986).

[Dea03] D. J. Dean, M. Hjorth-Jensen, "Pairing in nuclear systems: from neutron stars to finite nuclei," Rev. Mod. Phys. 75, 607-656 (2003).

[Deh68] H. G. Dehmelt, F. L. Walls, "Bolometric" technique for the rf spectroscopy of stored ions," Phys. Rev. Lett. 21, 127 (1968). 
[Del06] P. Delahaye et al., "High-accuracy mass measurements of neutron-rich Kr isotopes," Phys. Rev. C 74, 034331 (2006).

[Del10] J. P. Delaroche et al., "Structure of even-even nuclei using a mapped collective Hamiltonian and the D1S Gogny interaction," Phys. Rev. C 81, 014303 (2010).

[Dio74] J. S. Dionisio, C. Vieu, presented at the International conference on nuclear structure and spectroscopy, Amsterdam, 1974.

[Dob01] J. Dobaczewski, W. Nazarewicz, P.-G. Reinhard, "Pairing interaction and selfconsistent densities in neutron-rich nuclei," Nucl. Phys. A 693, Radioactive Nuclear Beams, 361-373 (2001).

[Dob09a] J. Dobaczewski et al., "HFODD (v2.40h) User's Guide," (2009).

[Dob09b] J. Dobaczewski et al., "Solution of the Skyrme-Hartree-Fock-Bogolyubov equations in the Cartesian deformed harmonic-oscillator basis.: (VI) HFODD (v2.40h): A new version of the program," Comp. Phys. Commun. 180, 23612391 (2009).

[Dob11] J. Dobaczewski, "Current Developments in Nuclear Density Functional Methods," J. Phys. Conf. Series 312, 092002 (2011).

[Dob97] J. Dobaczewski, J. Dudek, "Solution of the Skyrme-Hartree-Fock equations in the Cartesian deformed harmonic oscillator basis II. The program HFODD," Comp. Phys. Commun. 102, 183-209 (1997).

[Dug01] T. Duguet, P. Bonche, P.-H. Heenen, J. Meyer, "Pairing correlations. I. Description of odd nuclei in mean-field theories," Phys. Rev. C 65, 014310 (2001).

[Edd20] A. S. Eddington, "The Internal Constitution of the Stars," Nature 106, 14-20 (1920).

[Epe09] E. Epelbaum, H.-W. Hammer, U.-G. Meißner, "Modern theory of nuclear forces," Rev. Mod. Phys. 81, 1773-1825 (2009).

[Erl12] J. Erler et al., "The limits of the nuclear landscape," Nature 486, 509-512 (2012).

[Fed12] V. N. Fedosseev et al., "Upgrade of the resonance ionization laser ion source at ISOLDE on-line isotope separation facility: New lasers and new ion beams," Rev. Sci. Instrum. 83, 020000 (2012).

[Fed79] P. Federman, S. Pittel, "Unified shell-model description of nuclear deformation," Phys. Rev. C 20, 820-829 (1979).

[Fed84] P. Federman, S. Pittel, A. Etchegoyen, "Quenching of the $2 p_{1 / 2}-2 p_{3 / 2}$ proton spin-orbit splitting in the Sr-Zr region," Phys. Lett. B 140, 269-271 (1984).

[Fin12] D. Fink et al., "Q Value and Half-Lives for the Double- $\beta$-Decay Nuclide ${ }^{110} \mathrm{Pd}, "$ Phys. Rev. Lett 108, 062502 (2012). 
[Fin13] D. Fink et al., "First application of the Laser Ion Source and Trap (LIST) for on-line experiments at ISOLDE," Nucl. Instrum. Meth. B: 317, 417-421 (2013).

[Fri04a] G. Fricke, K. Heilig, in Nuclear Charge Radii, ed. by H. Schopper (Springer Berlin Heidelberg, 2004), vol. 20, pp. 1-4.

[Fri04b] G. Fricke, K. Heilig, in Nuclear Charge Radii, ed. by H. Schopper (Springer Berlin Heidelberg, 2004), vol. 20, pp. 1-9.

[Gal12] A. T. Gallant et al., "New Precision Mass Measurements of Neutron-Rich Calcium and Potassium Isotopes and Three-Nucleon Forces," Phys. Rev. Lett. 109, 032506 (2012).

[Gam30] G. Gamow, "Mass Defect Curve and Nuclear Constitution," Proc. Roy. Soc. London A 126, 632-644 (1930).

[Gar05] J. Garcia-Ramos, K. Heyde, R. Fossion, V. Hellemans, S. De Baerdemacker, "A theoretical description of energy spectra and two-neutron separation energies for neutron-rich zirconium isotopes," eng, Eur. Phys. J. A 26, 221-225 (2005).

[Geo07a] S. George et al., "Ramsey Method of Separated Oscillatory Fields for HighPrecision Penning Trap Mass Spectrometry," Phys. Rev. Lett. 98, 162501 (16 Apr. 2007).

[Geo07b] S. George et al., "The Ramsey method in high-precision mass spectrometry with Penning traps: Experimental results," Int. J. Mass Spectrom. 264, 110121 (2007).

[Gor01] S. Goriely, "Nuclear Masses and the r- and p-Processes of Nucleosynthesis," English, Hyperfine Interact. 132, 105-114 (2001).

[Gor09] S. Goriely, N. Chamel, J. M. Pearson, "Skyrme-Hartree-Fock-Bogoliubov Nuclear Mass Formulas: Crossing the 0.6 MeV Accuracy Threshold with Microscopically Deduced Pairing," Phys. Rev. Lett. 102, 152503 (2009).

[Gor13] S. Goriely, N. Chamel, J. M. Pearson, "Further explorations of Skyrme-HartreeFock-Bogoliubov mass formulas. XIII. The 2012 atomic mass evaluation and the symmetry coefficient," Phys. Rev. C 88, 024308 (2013).

[Gra80] G. Gräff, H. Kalinowsky, J. Traut, "A direct determination of the proton electron mass ratio," English, Z. Phys. A 297, 35-39 (1980).

[Hak12] J. Hakala et al., "Precision Mass Measurements beyond ${ }^{132} \mathrm{Sn}$ : Anomalous Behavior of Odd-Even Staggering of Binding Energies," Phys. Rev. Lett. 109, 032501 (2012).

[Han70] P. Hansen et al., "Studies of the $\alpha$-active isotopes of mercury, gold and platinum," Nucl. Phys. A 148, 249-272 (1970). 
[Har09] J. C. Hardy, I. S. Towner, "Superallowed $0^{+} \rightarrow 0^{+}$nuclear $\beta$ decays: A new survey with precision tests of the conserved vector current hypothesis and the standard model," Phys. Rev. C 79, 055502 (2009).

[Hax49] O. Haxel, J. H. D. Jensen, H. E. Suess, "On the "Magic Numbers" in Nuclear Structure," Phys. Rev. 75, 1766 (1949).

[Her01] F. Herfurth et al., "A linear radiofrequency ion trap for accumulation, bunching, and emittance improvement of radioactive ion beams," Nucl. Instrum. Meth. A 469, 254-275 (2001).

[Hey11] K. Heyde, J. L. Wood, "Shape coexistence in atomic nuclei," Rev. Mod. Phys. 83, 1467-1521 (2011).

[Hey87] K. Heyde et al., "A shell-model description of $0^{+}$intruder states in even-even nuclei," Nucl. Phys. A 466, 189-226 (1987).

[Hog53] B. G. Hogg, H. E. Duckworth, "Evidence for a Region of Extra Nuclear Stability between the 82- and 126-Neutron Shells," Phys. Rev. 91, 1289-1290 (1953).

[Hoh64] P. Hohenberg, W. Kohn, "Inhomogeneous Electron Gas," Phys. Rev. 136, B864-B871 (1964).

[Hol12] J. D. Holt, T. Otsuka, A. Schwenk, T. Suzuki, "Three-body forces and shell structure in calcium isotopes," J. Phys. G 39, 085111 (2012).

[Hon04] M. Honma, T. Otsuka, B. A. Brown, T. Mizusaki, "New effective interaction for $p f$-shell nuclei and its implications for the stability of the $N=Z=28$ closed core," Phys. Rev. C 69, 034335 (2004).

[Iaf84] R. Iafigliola et al., presented at the 7th International Conference Atomic Masses and Fundamental Constants AMCO-7, p. 141.

[Jah37] H. A. Jahn, E. Teller, "Stability of Polyatomic Molecules in Degenerate Electronic States. I. Orbital Degeneracy," Roy. Soc. Lond. Proc. A 161, 220-235 (1937).

[Joh73] A. Johansson, B. Nyman, "The Decay of 190 Au," Phys. Scripta 8, 99 (1973).

[Kei95] M. Keim et al., "Laser-spectroscopy measurements of 72-96Kr spins, moments and charge radii," Nucl. Phys. A 586, 219-239 (1995).

[Kel03] A. Kellerbauer et al., "From Direct to Absolute Mass Measurements: A Study of the Accuracy of ISOLTRAP," Eur. Phys. J. D 22, 53 (2003).

[Kel86] J. Keller et al., "Cold fusion in symmetric 90Zr-induced reactions," Nucl. Phys. A 452, 173-204 (1986).

[Klu13] H.-J. Kluge, "Penning trap mass spectrometry of radionuclides," Int. J. Mass Spectrom. 349-350, 26-37 (2013). 
[Koh65] W. Kohn, L. J. Sham, "Self-Consistent Equations Including Exchange and Correlation Effects," Phys. Rev. 140, A1133-A1138 (1965).

[Kon95] M. König, G. Bollen, H.-J. Kluge, T. Otto, J. Szerypo, "Quadrupole Excitation of Stored Ion Motion at the True Cyclotron Frequency," Int. J. Mass Spectrom. 142, 95 (1995).

[Kos02] U. Köster, "Intense radioactive-ion beams produced with the ISOL method," Eur. Phys. J. A 15, 255-263 (2002).

[Kos05] U. Köster et al., "ISOLDE beams of neutron-rich zinc isotopes: yields, release, decay spectroscopy," AIP Conf. Proc. 798, 315-326 (2005).

[Kow12] M. Kowalska et al., "Trap-assisted decay spectroscopy with ISOLTRAP," Nucl. Instr. and Methods A 689, 102 (2012).

[Kre07] M. Kretzschmar, "The Ramsey method in high-precision mass spectrometry with Penning traps: Theoretical foundations," Int. J. Mass Spectrom. 264, 122-145 (2007).

[Kre13] S. Kreim et al., "Recent exploits of the ISOLTRAP mass spectrometer," Nucl. Instrum. Meth. B 317, 492-500 (2013).

[Kre14] S. Kreim et al., "Competition between pairing correlations and deformation from the odd-even mass staggering of francium and radium isotopes," Phys. Rev. C 90, 024301 (2014).

[Kug00] E. Kugler, "The ISOLDE facility," English, Hyperfine Interact. 129, 23-42 (2000).

[Kuh77] T. Kühl et al., "Nuclear Shape Staggering in Very Neutron-Deficient Hg Isotopes Detected by Laser Spectroscopy," Phys. Rev. Lett. 39, 180-183 (1977).

[Lie91] P. Lievens et al., "Nuclear ground state properties of 99Sr by collinear laser spectroscopy with non-optical detection," Phys. Lett. B 256, 141-145 (1991).

[Lit05] Y. Litvinov et al., "Mass measurement of cooled neutron-deficient bismuth projectile fragments with time-resolved Schottky mass spectrometry at the FRS-ESR facility," Nuc. Phys. A 756, 3-38 (2005).

[Lui12] R. Luis, J. Marques, T. Stora, P. Vaz, L. Zanini, "Optimization studies of the CERN-ISOLDE neutron converter and fission target system," Eur. Phys. J. A 48 (2012).

[Lun03] D. Lunney, J. M. Pearson, C. Thibault, Rev. Mod. Phys. 75, 1021 (2003).

[Mac01] R. Machleidt, "High-precision, charge-dependent Bonn nucleon-nucleon potential," Phys. Rev. C 63, 024001 (2001).

[Mad88] D. G. Madland, J. Nix, "New model of the average neutron and proton pairing gaps," Nucl. Phys. A 476, 1-38 (1988). 
[Maj05] F. G. Major, V. N. Gheorghe, G. Werth, Charged Particle Traps, Physics and Techniques of Charged Particle Field Confinement (Springer-Verlag, Berlin, Germany, 2005).

[Man13] V. Manea et al., "Collective degrees of freedom of neutron-rich $A \approx 100$ nuclei and the first mass measurement of the short-lived nuclide ${ }^{100} \mathrm{Rb}, "$ Phys. Rev. C 88, 054322 (2013).

[Mar08] M. Marie-Jeanne et al., "Towards a magnetic field stabilization at ISOLTRAP for high-accuracy mass measurements on exotic nuclides," Nucl. Instrum. Meth. A 587, 464-473 (2008).

[Mar09] N. Mãrginean et al., "Evolution of deformation in the neutron-rich krypton isotopes: The ${ }^{96}$ Kr nucleus," Phys. Rev. C 80, 021301 (2009).

[Mar13] B. Marsh et al., "New developments of the in-source spectroscopy method at RILIS/ ISOLDE," Nucl. Instrum. Meth. B 317, 550-556 (2013).

[May48] M. G. Mayer, "On Closed Shells in Nuclei," Phys. Rev. 74, 235-239 (1948).

[May49] M. G. Mayer, "On Closed Shells in Nuclei. II," Phys. Rev. 75, 1969-1970 (1949).

[May50] M. G. Mayer, "Nuclear Configurations in the Spin-Orbit Coupling Model. II. Theoretical Considerations," Phys. Rev. 78, 22-23 (1950).

[Mol95] P. Möller, J. Nix, W. Myers, W. Swiatecki, "Nuclear Ground-State Masses and Deformations," At. Data and Nucl. Data Tables 59, 185-381 (1995).

[Muk08] M. Mukherjee et al., "ISOLTRAP: An on-line Penning trap for mass spectrometry on short-lived nuclides," Eur. Phys. J. A 35, 1-29 (2008).

[Mye66] W. D. Myers, W. J. Swiatecki, "Nuclear masses and deformations," Nucl. Phys. 81, 1-60 (1966).

[Mye74] W. Myers, W. Swiatecki, "The nuclear droplet model for arbitrary shapes," Ann. Phys. 84, 186-210 (1974).

[Nai10] S. Naimi et al., "Critical-Point Boundary for the Nuclear Quantum Phase Transition Near $A=100$ from Mass Measurements of ${ }^{96,97} \mathrm{Kr}, "$ Phys. Rev. Lett. 105, 032502 (2010).

[Nie02] A. Nieminen et al., "On-Line Ion Cooling and Bunching for Collinear Laser Spectroscopy," Phys. Rev. Lett. 88, 094801 (2002).

[Now09] F. Nowacki, A. Poves, "New effective interaction for $0 \hbar \omega$ shell-model calculations in the sd-pf valence space," Phys. Rev. C 79, 014310 (2009).

[Ots10] T. Otsuka, T. Suzuki, J. D. Holt, A. Schwenk, Y. Akaishi, "Three-Body Forces and the Limit of Oxygen Isotopes," Phys. Rev. Lett. 105, 032501 (2010). 
[Pen10] L. Penescu, R. Catherall, J. Lettry, T. Stora, "Development of high efficiency Versatile Arc Discharge Ion Source at CERN ISOLDE," Rev. Sci. Instrum. 81, pages (2010).

[Pie01] S. C. Pieper, R. B. Wiringa, "Quantum Monte Carlo calculations of light nuclei," Annual Review of Nuclear and Particle Science 51, 53-90 (2001).

[Pov01] A. Poves, J. Sánchez-Solano, E. Caurier, F. Nowacki, "Shell model study of the isobaric chains $\mathrm{A}=50, \mathrm{~A}=51$ and $\mathrm{A}=52, "$ Nuclear Physics A 694, 157-198 (2001).

[Rai05] S. Rainville et al., "World Year of Physics: A direct test of $E=m c^{2}$," Nature 438, 1096-1097 (2005).

[Rai97] H. Raimbault-Hartmann et al., "A cylindrical Penning trap for capture, mass selective cooling, and bunching of radioactive ion beams," Nucl. Instrum. Methods B 126, 378-382 (1997).

[Ram01] S. Raman, C. N. Jr., P. Tikkanen, "Transition probability from the ground to the first-excited $2^{+}$state of even-even nuclides," At. Data Nucl. Data Tables 78, 1-128 (2001).

[Red13] M. Redshaw et al., "LEBIT II: Upgrades and developments for high precision Penning trap mass measurements with rare isotopes," Nucl. Instrum. Meth. B 317, 510-516 (2013).

[Rin00] P. Ring, P. Schuck, The Nuclear Many-body Problem (Springer, 2000).

[Rob11] L. M. Robledo, G. F. Bertsch, "Global systematics of octupole excitations in even-even nuclei," Phys. Rev. C 84, 054302 (2011).

[Rod10a] R. Rodríguez-Guzmán, P. Sarriguren, L. M. Robledo, "Signatures of shape transitions in odd- $A$ neutron-rich rubidium isotopes," Phys. Rev. C 82, 061302 (2010).

[Rod10b] R. Rodríguez-Guzmán, P. Sarriguren, L. Robledo, S. Perez-Martin, "Charge radii and structural evolution in Sr, Zr, and Mo isotopes," Phys. Lett. B 691, 202-207 (2010).

[Roo04] J. Roosbroeck et al., "Unambiguous Identification of Three $\beta$-Decaying Isomers in ${ }^{70} \mathrm{Cu}$," Phys. Rev. Lett. 92, 112501 (2004).

[Ros14] M. Rosenbusch et al., "Ion bunch stacking in a Penning trap after purification in an electrostatic mirror trap," English, Appl. Phys. B 114, 147-155 (2014).

[Rut19] E. Rutherford, "LIV. Collision of $\alpha$ particles with light atoms. IV. An anomalous effect in nitrogen," Phil. Mag. 37, 581-587 (1919).

[Rut99] K. Rutz, M. Bender, P.-G. Reinhard, J. Maruhn, "Pairing gap and polarisation effects," Phys. Lett. B 468, 1-6 (1999). 
[Sav90] G. Savard et al., "Laser spectroscopy of laser-desorbed gold isotopes," Nucl. Phys. A 512, 241-252 (1990).

[Sav91] G. Savard et al., "A new cooling technique for heavy ions in a Penning trap," Phys. Lett. A 158, 247-252 (1991).

[Sch10] N. Schunck et al., "One-quasiparticle states in the nuclear energy density functional theory," Phys. Rev. C 81, 024316 (2010).

[Sch12] N. Schunck et al., "Solution of the Skyrme-Hartree-Fock-Bogolyubov equations in the Cartesian deformed harmonic-oscillator basis.: (VII) HFODD (v2.49t): A new version of the program," Comp. Phys. Commun. 183, 166-192 (2012).

[Sch37] T. Schmidt, "Über die magnetischen Momente der Atomkerne," Zeit.Phys. 106, 358-361 (1937).

[Sch86] H. Schnatz et al., "In-flight capture of ions into a penning trap," Nucl. Instrum. Meth. A 251, 17-20 (1986).

[Sii68] A. Siivola, "Alpha-active gold isotopes," Nucl. Phys. A 109, 231-235 (1968).

[Sim12] V. V. Simon et al., "Penning-trap mass spectrometry of highly charged, neutronrich $\mathrm{Rb}$ and $\mathrm{Sr}$ isotopes in the vicinity of $A \approx 100, "$ Phys. Rev. C 85, 064308 (2012).

[Som14] V. Somà, C. Barbieri, T. Duguet, "Ab initio self-consistent Gorkov-Green's function calculations of semi-magic nuclei: Numerical implementation at second order with a two-nucleon interaction," Phys. Rev. C 89, 024323 (2014).

[Sor08] O. Sorlin, M.-G. Porquet, "Nuclear magic numbers: New features far from stability," Progr. Part. Nucl. Phys. 61, 602 (2008).

[Sta13] J. Stanja et al., "Mass spectrometry and decay spectroscopy of isomers across the $Z=82$ shell closure," Phys. Rev. C 88, 054304 (2013).

[Ste13] D. Steppenbeck et al., "Evidence for a new nuclear 'magic number' from the level structure of ${ }^{54} \mathrm{Ca}, "$ Nature 502, 207-210 (2013).

[Sto11] N. J. Stone, "Table of Nuclear Magnetic Dipole and Electric Quadrupole Moments," Int. Nucl. Data Committee, document INDC(NDS)-0594 (2011).

[Sto94] V. G. J. Stoks, R. A. M. Klomp, C. P. F. Terheggen, J. J. de Swart, "Construction of high-quality NN potential models," Phys. Rev. C 49, 2950-2962 (1994).

[Str67] V. Strutinsky, "Shell effects in nuclear masses and deformation energies," Nucl. Phys. A 95, 420-442 (1967).

[Stu12] A. E. Stuchbery, "Simple structures in complex nuclei versus complex structures in simple nuclei: a nuclear moments perspective," J. Phys.: Conf. Series 366, 012042 (2012). 
[Sum11] T. Sumikama et al., "Structural Evolution in the Neutron-Rich Nuclei ${ }^{106} \mathrm{Zr}$ and ${ }^{108} \mathrm{Zr}, "$ Phys. Rev. Lett. 106, 202501 (2011).

[Tha03] H. L. Thayer et al., "Collinear laser spectroscopy of radioisotopes of zirconium," J. Phys. G 29, 2247 (2003).

[Thi81] C. Thibault et al., "Hyperfine structure and isotope shift of the $D_{2}$ line of ${ }^{76-98} \mathrm{Rb}$ and some of their isomers," Phys. Rev. C 23, 2720-2729 (1981).

[Ulm13] S. Ulmer et al., "A cryogenic detection system at $28.90 \mathrm{MHZ}$ for the nondestructive observation of a single proton at low particle energy," Nuc. Instrum. Meth. A 705, 55-60 (2013).

[Ulm86] G. Ulm et al., "Isotope shift of ${ }^{182} \mathrm{Hg}$ and an update of nuclear moments and charge radii in the isotope range ${ }^{181} \mathrm{Hg}_{-}{ }^{206} \mathrm{Hg}$," Z. Phys. A 325, 247-259 (1986).

[Vie76] C. Vieu, J. S. Dionisio, V. Berg, C. Bourgeois, presented at the International Conference on Nuclei Far from Stability 3, Cargese, France, 1976.

[Vou08] D. Voulot et al., "Radioactive beams at REX-ISOLDE: Present status and latest developments," Nucl. Instrum. Meth. B 266, 4103-4107 (2008).

[Wal87] K. Wallmeroth et al., "Sudden change in the nuclear charge distribution of very light gold isotopes," Phys. Rev. Lett. 58, 1516-1519 (1987).

[Wal89] K. Wallmeroth et al., "Nuclear shape transition in light gold isotopes," Nucl. Phys. A 493, 224-252 (1989).

[Wan12] M. Wang et al., "The AME2012 atomic mass evaluation (II). Tables, graphs, and references," Chinese Phys. C 36, 1603 (2012).

[Was12] K. Washiyama et al., "New parametrization of Skyrme's interaction for regularized multireference energy density functional calculations," Phys. Rev. C 86, 054309 (2012).

[Wau93] J. Wauters et al., "Alpha-decay study of188Pb and180,182Hg," Zeit. Phys. A 345, 21-27 (1993).

[Wei35] C. Weizsäcker, "Zur Theorie der Kernmassen," Zeit. Phys. 96, 431-458 (1935).

[Wer09] G. Werth, V. N. Gheorghe, F. G. Major, Charged Particle Traps II, Applications (Springer-Verlag, Berlin, Germany, 2009).

[Wid08] E. Widmann, in Trapped Charged Particles and Fundamental Interactions, ed. by K. Blaum, F. Herfurth (Springer, Heidelberg, Germany, 2008), vol. 749, pp. $155-188$.

[Wie13] F. Wienholtz et al., "Masses of exotic calcium isotopes pin down nuclear forces," Nature 498, 346 (2013).

[Wig37] E. Wigner, "On the Consequences of the Symmetry of the Nuclear Hamiltonian on the Spectroscopy of Nuclei," Phys. Rev. 51, 106-119 (1937). 
[Wir95] R. B. Wiringa, V. G. J. Stoks, R. Schiavilla, "Accurate nucleon-nucleon potential with charge-independence breaking," Phys. Rev. C 51, 38-51 (1995).

[Wit07] H. Witte et al., "Nuclear Charge Radii of Neutron-Deficient Lead Isotopes Beyond $N=104$. Midshell Investigated by In-Source Laser Spectroscopy," Phys. Rev. Lett. 98, 112502 (2007).

[Wol03] B. Wolf et al., "First radioactive ions charge bred in REXEBIS at the REXISOLDE accelerator," Nucl. Instrum. Meth. B 204, 14th International Conference on Electromagnetic Isotope Separators and Techniques Related to their Applications, 428-432 (2003).

[Wol12] R. Wolf et al., "On-line separation of short-lived nuclei by a multi-reflection time-of-flight device," Nucl. Instrum. Meth. A 686, 82-90 (2012).

[Wol13a] R. N. Wolf et al., "Plumbing Neutron Stars to New Depths with the Binding Energy of the Exotic Nuclide ${ }^{82} Z$,", Phys. Rev. Lett. 110, 041101 (2013).

[Wol13b] R. Wolf et al., "ISOLTRAP's multi-reflection time-of-flight mass separator/ spectrometer," Int. J. Mass Spectrom. 349-350, 123-133 (2013).

[Yuk35] H. Yukawa, "On the Interaction of Elementary Particles," Proc. Phys. Math. Soc. Jap. 17, 48 (1935).

[Zum62] B. Zumino, "Normal Forms of Complex Matrices," J. Math. Phys. 3, 10551057 (1962). 